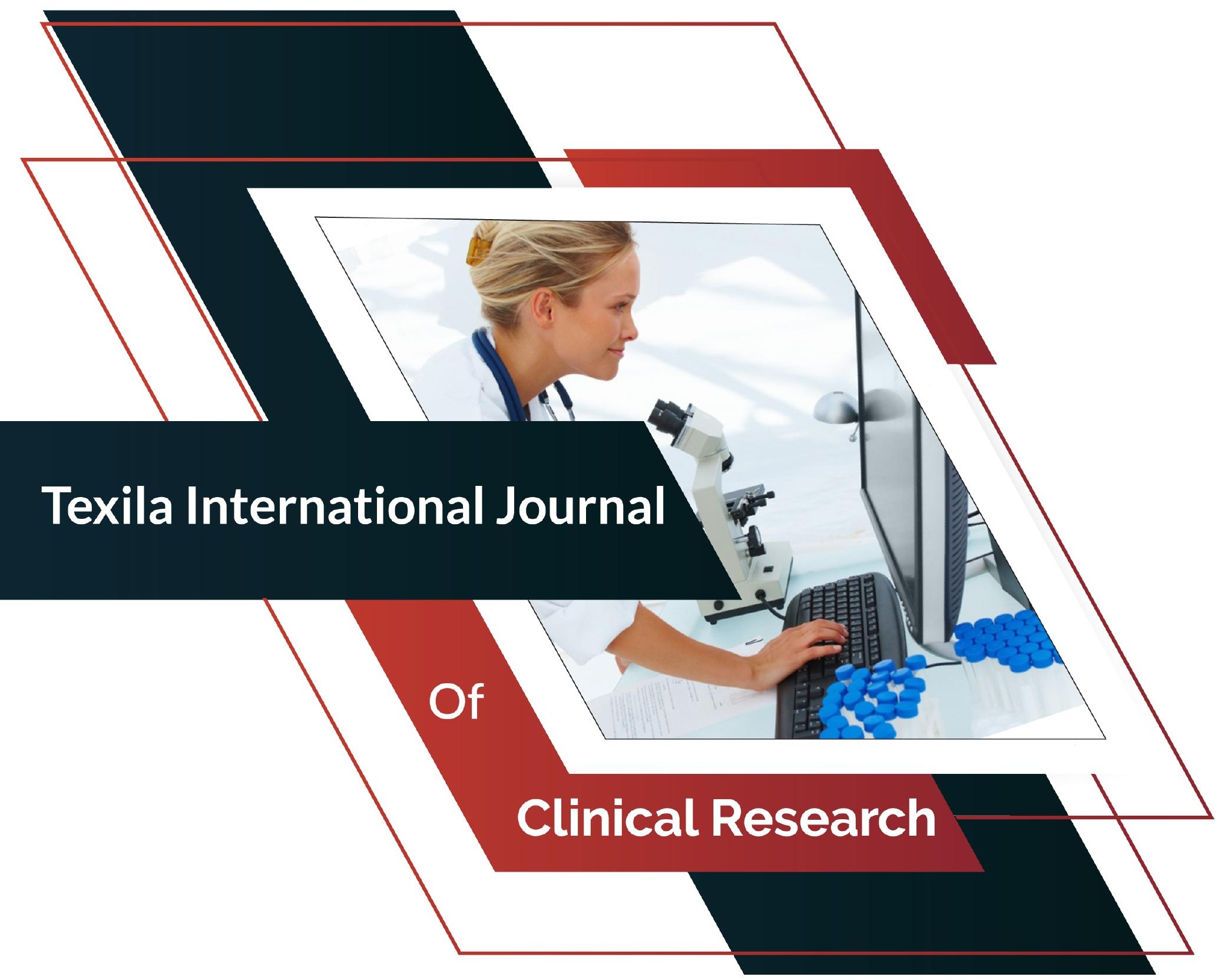

Volume 3, Issue 2

December 2016 


\section{EDITORIAL POLICY}

Papers must be submitted with the understanding that they have not been published elsewhere (except in the form of an abstract or as part of a published lecture, review, or thesis) and are not currently under consideration by another journal published or any other publisher. The submitting (Corresponding) author is responsible for ensuring that the article's publication has been approved by all the other coauthors. It is also the authors' responsibility to ensure that the articles coming from a particular institution are submitted with the approval of the necessary institution. Only an acknowledgment from the editorial office officially establishes the date of receipt. It is a condition for submission of a paper that the authors permit editing of the paper for readability. All enquiries concerning the publication of accepted papers should be addressed to ejournal.assist@tau.edu.gy. 


\section{ABOUT PLAGIARISM}

Plagiarism is the use or close imitation of the language and ideas of another author and representation of them as one's own original work. Duplicate publication, sometimes called self plagiarism, occurs when an author reuses substantial parts of his or her own published work without providing the appropriate references. This can range from getting an identical paper published in multiple journals, where authors add small amounts of new data to a previous paper.

Plagiarism can be said to have clearly occurred when large chunks of text have been cut and pasted. Such manuscripts would not be considered for publication in TIJBMS Journal. But minor plagiarism without dishonest intent is relatively frequent, for example when an author reuses parts of an introduction from an earlier paper. The editors will judge any case of which they become aware (either by their own knowledge of and reading about the literature, or when alerted by referees) on its own merits.

The paper containing the plagiarism will be obviously returned back to the author's for review, but we earnestly request the authors to avoid submitting plagiarized 


\section{DISCLAIMER}

Texila International Journal of Clinical Research (TIJCR) make every effort to ensure the accuracy of all the information (the "Content") contained in its publications. However, the TIJCR and its agents make no representations or warranties whatsoever as to the accuracy, completeness or suitability for any purpose of the Content and disclaim all such representations and warranties whether express or implied to the maximum extent permitted by law. Any views expressed in this publication are the views of the authors and are not necessarily the views of the Editor's or Texila International Journal of Clinical Research. 


\section{TABLE OF CONTENT}

1 Anxiety-Depression Affect on Quality of Life of Cancer patients during

Chemotherapy and Radiotherapy Treatment

Kavita Gupta

2 Diabetes Education Program Increases Knowledge, Reduces Obesity and better Glycemic Control in Central Hospital Nampula

Madhumati Varma

3 Mitigating Lung Infections in Poor HIV Patients

Lois Collie-Acasio

4 Prevalence and Management of Rhinosinusitis in Developing Countries: the

Case of Mbingo Baptist Hospital, North West Region, Cameroon

Tcheuwebe Liale Honore

5 Break the Germ Cycle: Hand Hygiene Compliance among Healthcare

Workers in an Accredited Tertiary Care Hospital

Jessy Sebastian

6 Review of updates on the prevalence and epidemiology of Acetaminophen

89 poisoning in the United States and the United Kingdom

Njoku Amara Chiemezie, L.

7 Impact of Regulatory Amendment on Human Subjects, Clinical Research Industries and its stakeholder in India

Kapil Krishna Gupta

8 Adverse Drug Reaction Reporting By Doctors in a Developing Country: a Case Study from Ghana

Mark Korankye

9 An overview of the importance of the Trial Master File (TMF) and the required contents in clinical trials as stipulated in the ICH GCP guidelines 
Catharina Susanna Venter

10 Adherence to antiretroviral therapy among people living with HIV and AIDS

in Monze and Nyimba districts of Zambia

Emmanuel Mwila Musenge

\begin{tabular}{rr}
\hline 11 Acute kidney Injury: Prevalence, Diagnosis, Causes and treatment & 133 \\
Alermayehu Lelisa Duga &
\end{tabular}

12 Counselling and Moral Education: A Panacea in Curbing the Increasing

Trends of Agitation in Nigeria

Victoria Uzoamaka Ezeh

13 The State of Mental Health in South Africa Cross Examination of the Vicious Cycle of the Demographic and Socio-Economic Factors that Affect Mental Health

Sakibu Lyaga

14 Review on Multiple Ethical Review in North-South Collaborative Research:

165 the Experience of the Ebola-Tx Trial in Guinea

Labban Fredrick Sichivula

15 Effect of Structured In-service Education on Knowledge level among Nurses Working in Surgical Units in Private Tertiary Hospital Islamabad, Pakistan

Nuzhat Sultana

16 Standardization of Herbal Medicine and Enforcement of Regulations in Herbal Medical System

\section{Saveri Raj P.X}

17 The Prevalence of Needle Stick/Sharp Objects Injury in Hospital Staff and Preventive Practices Taken into Consideration

Badmus Omobolanle Tawakalit

18 Patient's ability to consent during Inpatient Chemotherapy Treatment or Outpatient Radiotherapy Treatment in a tertiary care hospital of New Delhi, India

Kavita Gupta

19 Anxiety \& Depression Assessment of cancer patients undergoing Chemotherapy or Radiotherapy Treatment by the use of computerized developed version of Questionnaire (WHOQOL-Bref, ZSAS, ZSDS)

\section{Kavita Gupta}

20 Sexual Health, Suicidal Ideation and weight changes in Cancer patients during Chemotherapy and Radiotherapy Treatment 
Kavita Gupta

21 Patient's perspective of QOL with respect to different domains of Quality of 228 Life in Cancer patients during inpatient Chemotherapy Treatment or outpatient Radiotherapy Treatment in a tertiary care hospital of New Delhi, India

\author{
Kavita Gupta
}




\title{
Anxiety-Depression Affect on Quality of Life of Cancer patients during Chemotherapy and Radiotherapy Treatment
}

\author{
Article by Kavita Gupta \\ Ph.D Clinial Research, Texila American University, India \\ E-mail: 16kavitagupta@texilaconnect.com
}

\begin{abstract}
Background: Potentially every aspect of life in cancer patients is found to be accompanied by a mental illness of anxiety and depression that affects the Physical relationships, Psychological wellbeing, Social relationships and the Environmental domain thereby reducing the QOL of cancer patients significantly. The advancements in cancer treatment have led to marked improvement in the survival rates of cancer patients; however, distressing symptoms of anxiety and depression arising due to treatment modalities are yet to be endured. The proposed study is considered valuable since it emphasized on the quality of life of cancer patients during their treatment for cancer symptoms as one of the possible outcomes. Because patients are the best source of information for QOL data, therefore inpatients for chemotherapy treatment and outpatients for radiotherapy treatment were recruited in the study to obtain a unique perspective on how QOL is defined, assessed, and perceived with respect to anxiety and depression during the treatment sessions.

Objectives: The present study was conducted to assess the association and correlation of Anxiety and Depression with the domains of Quality of Life of cancer patients during inpatient Chemotherapy sessions or outpatient Radiotherapy sessions. The primary objective was to analyze the anxiety and depression levels (Recognition patterns) with their impact on QOL of cancer patients during chemotherapy or radiotherapy sessions.

Methods: It was a Psychological intervention, non-experimental, cross-sectional, descriptive, observational, hospital-based evaluation study. The Research study was conducted at Medical and Radiation Oncology department of Dr. B.L. Kapur Memorial Hospital, New Delhi, India with total duration of 5 months (December 2015- April 2016). A convenient sample of 60 patients undergoing cancer treatment was selected in which 30 cancer patients were on chemotherapy (CT Group) and the other 30 cancer patients were on radiotherapy (RT Group). Data collection was performed by using 3 validated Psychological interventional tools: WHOQOL-Bref Questionnaire, Zung Self-Rating Anxiety scale (ZSAS) and Zung Self-Rating Depression scale (ZSDS). Appropriate statistical tests by using SPSS v.17 software were utilized to determine the significant association of anxiety and depression with QOL domains.

Results: As a result, it was observed that 21(35\%) males [6(20\%) in Chemotherapy group and 15(50\%) in Radiotherapy group] and 39(65\%) females [24(80\%) in Chemotherapy group and 15(50\%) in Radiotherapy group] participated in the research study. Majority of the patients $32(53.34 \%)$ were found to be in the age range of 46-60 years. Out of the enrolled subjects, 33(55\%) were undergoing Breast cancer treatment, 6(10\%) were undergoing Head cancer treatment, and 21(35\%) were undergoing Neck cancer treatment. Anxiety affected the Physical domain ( $p=0.007)$, and Environmental domain $(p=0.036)$ in the Chemotherapy group; and Social domain $(p=0.016)$ in the Radiotherapy group. On the other hand, Depression affected the Social domain $(p=0.043)$ in Chemotherapy group; and Social domain $(p=0.012)$ in the Radiotherapy group. Values of $p<0.05$ were considered to be statistically significant.

Conclusion: It is evident from the research study that Cancer patients on chemotherapy were more badly affected in some domains of Quality of life due to anxiety and depression as compared to the patients on radiotherapy treatment regimen. Moreover, it was observed that with the increase in Anxiety and Depression symptomatic levels, there was a marked decline in respective domains of the
\end{abstract}


Texila International Journal of Clinical Research

Volume 3, Issue 2, Dec 2016

Quality of life of the cancer patients during Chemotherapy and Radiotherapy sessions. Thus, it was observed that levels of Anxiety and Depression were inversely (negatively) correlated with the domains of Quality of life of cancer patients during chemotherapy or radiotherapy.

Descriptors: WHOQOL-Bref Rating scale; Zung Self-Rating Anxiety scale (ZSAS); Zung SelfRating Depression scale (ZSDS); Quality of Life; Sample study evaluation; Cancer patients; Chemotherapy treatment; Radiotherapy Treatment.

Identifiers: Anxiety (ZSAS), Depression (ZSDS), \& WHOQOL-Bref Profile of cancer patients during Chemotherapy \& Radiotherapy

Population: AdultCancer Patients undergoing either Chemotherapy or Radiotherapy sessions

Independent variables: Anxiety, Depression, Socio-demographic \& Clinical characteristics

Dependent variables: Physical health, Psychological well-being, Social relationships, Environment domain

Outcome measure: Quality of Life of cancer patients during Chemotherapy \& Radiotherapy

Domain: QOL as a characteristic function of Anxiety and Depression

Type of Measure: Subject-reported, Care-giver-reported, Investigator reported

Keywords: Cancer, Chemotherapy, Radiotherapy, Quality of Life, WHOQOL-Bref Questionnaire, Zung Self-Rating Anxiety scale, Zung Self-Rating Depression scale, Physical domain, Psychological domain, Social domain, Environmental domain.

$\begin{array}{lll}\text { Abbreviations } & & \\ \text { QOL } & : & \text { Quality of life } \\ \text { QLQ } & : & \text { Quality life Questionnaire } \\ \text { WHOQOL-Bref } & : & \text { World Health Organization Quality of life assessment-a short brief } \\ & & \text { version } \\ \text { ZSAS } & : & \text { Zung Self-Rating Anxiety scale } \\ \text { ZSDS } & : & \text { Zung Self-Rating Depression scale } \\ \text { CT } & : & \text { Chemotherapy Treatment } \\ \text { RT } & : & \text { Radiotherapy Treatment } \\ \text { DOM } & : & \text { Domain } \\ \text { TPA } & : & \text { Third Party Administrator } \\ \text { FNAC } & : & \text { Standard deviation. }\end{array}$

\section{Introduction}

Cancer is defined as a generic term for a large group of chronic diseases that is known to be pathologically characterized by abnormal rapid growth of cells thereby affecting any part of the human body of both genders, and thus have become a major cause of mortality and morbidity worldwide ${ }^{[2,10]}$. According to the World Cancer Report, it is expected that Cancer rates would increase by $50 \%$ new cases for the year $2020^{[8]}$. According to GLOBOCAN report, it was estimated that about 14.1 million new cancer cases and 8.2 million deaths occurred in 2012 worldwide that included approximately 300,400 new cases and 145,400 of deaths from oral cavity cancer (including lip cancer), and approximately 86,700 new cases and 50,800 deaths from Nasopharyngeal cancer with more prevalence in males as compared to females ${ }^{[21]}$. The incidence of cancer in Delhi is the fourth highest among the Asian registries ${ }^{[6]}$. According to National Cancer Registry Program (NCRP) 2013 report, cancer is a threatening problem in India with an estimated 2.5 million people living with the disease with 19746 cases (29.8 \% of all cancer in men and $10.6 \%$ of all cancer in women) in Delhi alone ${ }^{[7,16]}$. Clinically, there are 3 available methods to treat and manage cancer-related symptoms that are namely, surgery, chemotherapy and radiotherapy which is being planned according to the patient's condition, site and stage of tumor followed by current guided protocol ${ }^{[2,10]}$. Chemotherapy is defined as a concentrated and repeated treatment drug regimen for the management of cancer and its related 
symptoms, which is found to be associated with adverse reactions such as, hair loss, nausea, vomiting, and diarrhea, thereby leading to extended periods of treatment and repeated admissions to the hospital eventually affecting the QOL of cancer patients ${ }^{[14]}$. On the other hand, Radiation therapy which is often the final step in the multimodal treatment regimen for cancer might cause side-effects such as, skin pigmentation, destruction of salivary glands, severe problems related to eating, swallowing and speech, causing xerostomia, oral infections, dental caries, pain and discomfort ${ }^{[3,9]}$. Many aspects of QOL related to chemotherapy or radiotherapy treatment have been studied [1, 22]. Patients often experience treatment-related (Chemotherapy or Radiotherapy) adverse effects (fatigue, anxiety, pain, lymphedema, neuropathy, cardiotoxicity, concern for the future and the family, difficulties to meet basic demands and changes in body image, sleep disturbances, and cognitive problems) that negatively affects physical, psychological, social, and environmental aspects or domains of quality of life ultimately leading to negative health consequences ${ }^{[1,22]}$. Therefore, this whole routine of chemotherapy and radiotherapy to "take care of cancer" could change dramatically the patients' everyday life, interfering with their quality of life ${ }^{[5,15]}$. Thus, Quality of Life is a general term integrating several aspects of life such as patients' physical (movement, physical activities, ability to succeed in work and in family responsibilities), psychological (life satisfaction), social (social activities, being beneficial, body image, anxiety and depression, social support need and role function), economic, spiritual, cognitional \&environmental dimensions for well-being during the diseased and respective-treatment stage ${ }^{[4,20]}$. Disturbance in any one of these aspects could in turn affect the other domains and this influences the overall Quality of Life ${ }^{[20]}$.

Importance of QOL measurement for clinical practice in cancer:

a. It could help patients in overviewing their treatment related side-effects and recovery trajcetory. On the other hand, it could help clinicians to make treatment decisions and evaluate therapeutic interventions. On the whole, it could give an accurate picture of the patient's overall benefit from the whole service ${ }^{[11]}$.

b. It could help to identify the individual patient's needs for additional supportive interventions such as perceived social support, employment creative interventions, introduction of psychotherapy as integral part of the treatment in order to, manage the symptoms of the disease as well as, treatment ${ }^{[11]}$.

Anxiety and Depression can be defined asuneasiness, nervousness, worry, or fear (not knowing what to expect or knowing what to expect), is a disturbed feeling often experienced during chemotherapy or radiotherapy sessions thereby affecting the QOL of cancer patients in daily activities $^{[4,5,11,15,20]}$.

\section{Aims}

The main objective of the present study was:

i. To determine the association and relationship of anxiety and depression with different domains of quality of life in cancer patients during chemotherapy treatment or radiotherapy treatment.

ii. To evaluate anxiety and depressive symptoms by the use of WHOQOL-Bref, Zung Self-rating Anxiety Scale (ZSAS) and Zung Self Rating Depression Scale (ZSDS) questionnaire in cancer patients receiving either Chemotherapy or Radiotherapy treatment regimen.

\section{Problem statement}

To conduct a evaluation and observation-based research study to determine the association of Anxiety \& Depression symptoms with Physical, Psychological, Social, and Environment domains of QOL of cancer patients during Chemotherapy and Radiotherapy by the Psychological intervention tool of WHOQOL-Bref, ZSAS \& ZSDS.

\section{Patients and methods}

The proposed study was conducted in accordance and adherence to the Ethical Guidelines and Procedures. Special care of the potential risks due to emotional distress was taken care of so that the dignity of the subject was not harmed. The authorized Ethical approval from the IRB and Ethical 
Texila International Journal of Clinical Research

Volume 3, Issue 2, Dec 2016

committee ofDr. B.L. Kapur Memorial Hospital, New Delhi, with Ref. No.: IRB/AARCE/5/DEC/2015/1, dated December $7^{\text {th }}$, 2015, was obtained to carry out the research study. Thereafter, the patients and their caregivers were approached in the inpatient as well as, outpatient clinic, where the purpose of the study was explained and they were invited to participate. Patients who agreed to participate were asked to sign an Informed Consent Form followed by the implementation of the Structured and Validatedinstrumental tool of WHOQOL-Bref, Zung Self-Rating Anxiety Scale (ZSAS), and Zung Self-Rating Depression Scale (ZSDS) in the form of questionnaire which lasted for approximately 25-60 minutes. The RESEARCH DESIGN of the proposed approved study protocol included 30n cancer patients undergoing Chemotherapy Treatment and 30n cancer patients undergoing Radiotherapy Treatment session. The inclusion criteria for the approved study were the patients with Breast cancer, sub-sites of head and neck tumors (e.g., nasopharyngeal, thyroid cancer, and parotid tumors), aged 18years or older, Clinically diagnosed and confirmed by biopsy or FNAC, Undergoing/during the treatment sessions ( $\geq 2$ cycles and $\leq 6$ for Chemotherapy treatment, and $\geq 10$ cycles and $\leq 30$ cycles for Radiotherapy treatment, Voluntarily agreed to join the study, and aware of diagnosis and predicted prognosis. However, the study excluded patients with Inadequate clinical condition (ambulatory and terminally ill patients) who were unable to respond to an interview, had difficulty in understanding the questionnaire or communicating, were serious and didn't give consent, had a history of psychiatric disorder. Moreover, the study did not include the dosage of chemotherapy drugs and irradiation treatment. Eligible patients were identified through an institutional database or by referring physicians and were approached at their simulation appointment. 


\section{Flowchart of the Research Study}

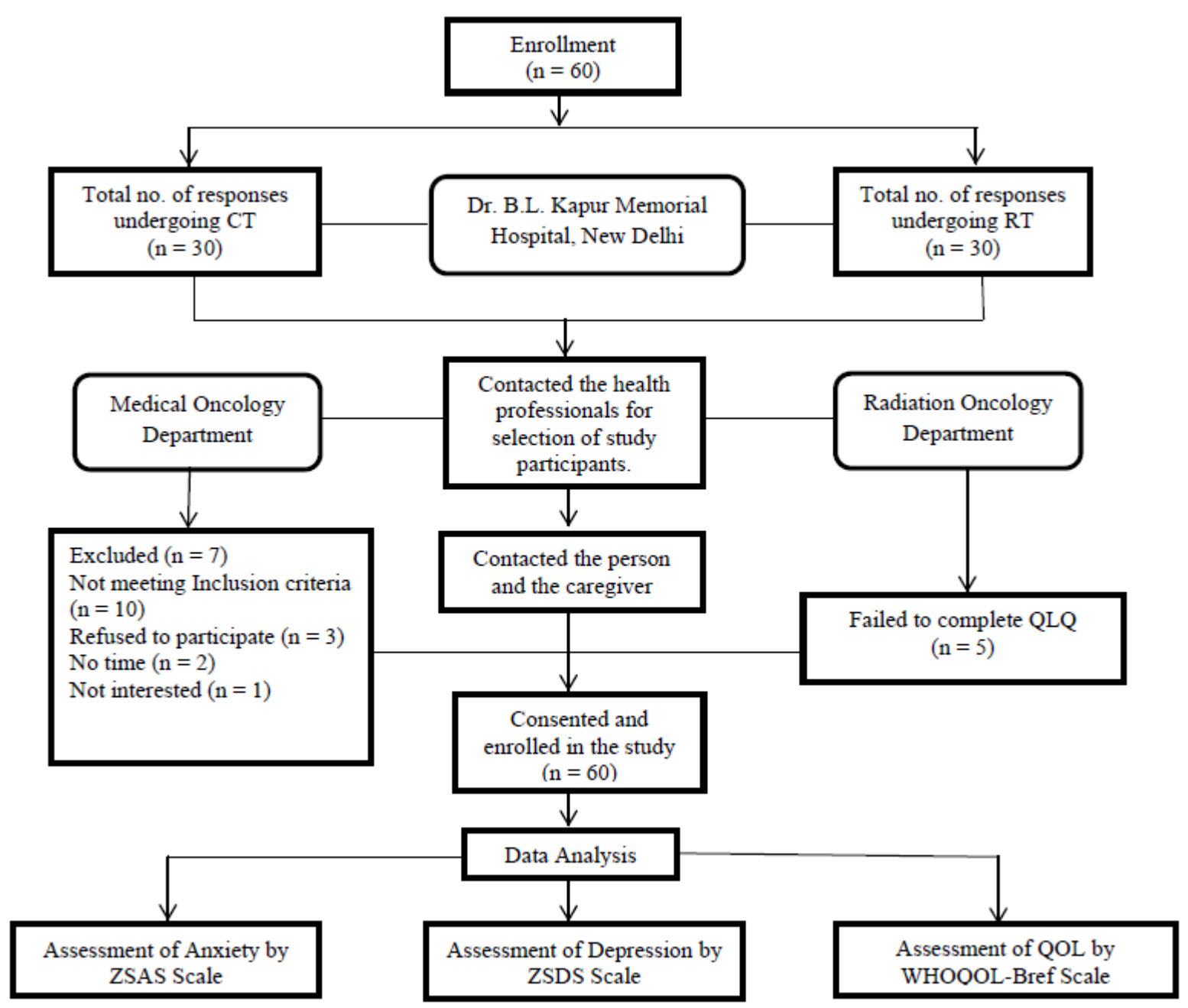

Fig.1: Flowchart of the research study after IRB and ethical approval

\section{Results}

\section{(a) Data collection}

Treatment-related symptoms were assessed by using a series of interviews through standard questionnaires of WHOQOL-Bref, the core questionnaire, followed by Zung Self-Rating Anxiety Scale (ZSAS) and Zung Self Rating Depression Scale (ZSDS) Questionnaires. These questionnaires have been proven to have good validity and reliability properties, cross-culturally accepted instrument to measure Quality of Life in cancer patients and are publicly available for scholar research purposes [23, 24, 25]. The questionnaire was provided in a language that the patient could understand easily (English / Hindi) followed by face-to-face interview of the patient who was either undergoing chemotherapy or radiotherapy treatment sessions.

Parts of the Record Card:

i. Personal information form:This first part contained patient information. The form was prepared, based on the literature. It contained age, gender, qualification, marital status, family type, Occupation, data on financial income and site of tumor location ${ }^{[23]}$.

ii. WHOQOL-Bref Questionnaire: This is an abbreviated version of the instrument WHOQOL-100. It consisted of 26 questions, being two about quality of life in general and other 24 representing 
each of the facets that made up the original instrument ${ }^{[23]}$. The questions were organized in 4 domains:

a. Physical domain (DOM1): It included7 questions pertaining to sleep, energy, mobility, the extent to which pain prevents performance of necessary tasks, the need for medical treatment to function in daily life, level of satisfaction with their capacity for work ${ }^{[23]}$.

b. Psychological domain (DOM2): It included6 questions pertaining to the ability to concentrate, self-esteem, body image, spirituality i.e. the extent to which they feel their life is meaningful, the frequency of positive or negative feelings i.e. blue mood, despair, anxiety, depression ${ }^{[23]}$.

c. Social domain (DOM3): It included 3 questions pertaining to satisfaction with personal relationships, social support systems and sexual satisfaction ${ }^{[23]}$.

d. Environmental domain (DOM4): It included 8 questions related to safety and security, home and physical environment satisfaction, finance i.e. does the respondent have enough money to meet their needs, health/social care availability, information and leisure activity accessibility and transportation satisfaction ${ }^{[23]}$.

Equations for computing domain raw scores:

Domain 1 (Physical) score $=(6-\mathrm{Q} 3)+(6-\mathrm{Q} 4)+\mathrm{Q} 5+\mathrm{Q} 6+\mathrm{Q} 7+\mathrm{Q} 8+\mathrm{Q} 9$

Domain 2 (Psychological) score = Q10 + Q11 + Q12 + Q13 + Q14 + (6-Q15)

Domain 3 (Social) score $=\mathrm{Q} 16+\mathrm{Q} 17+\mathrm{Q} 18$

Domain 4 (Environmental) score = Q19+Q20 + Q21 + Q22 + Q23 + Q24 + Q25 + Q26 ${ }^{[23]}$.

In addition to the 4 domains, the WHOQOL-Bref included two stand-alone questions, one pertaining to the respondents' rated QOL, and one related to their Satisfaction with Health were analyzed separately ${ }^{[23]}$. The score of each question ranged from 1 to 5 on a 5 point likert scale and higher scores indicated a better evaluation. Raw scores of the respective domains were then transformed from 0-100 with the lowest score of zero and the highest score of 100according to the accepted guidelines.

iii.ZSAS: Zung Self-Rating Anxiety scale quality life questionnaire is a likert scale format (scoring on 1 to 4 scale) that was built by a psychiatrist, William W. K. Zung to measure the rate of anxiety. The scale consisted of 20 self-reported items with 15 questions of increasing anxiety level and 5 questions of decreasing anxiety level (Q. no. 5, 9, 13, 17, 19) ${ }^{[24]}$. Scores for each question ranged from 1 to 4 and higher scores indicated severe anxiety level. The raw scores were counted up and multiplied by 1.25 to reach a standardized score, according to the instructions that accompanied the scale ${ }^{[24]}$. The ZSAS Index score followed the crierion: Normal Range (20-44); Mild to Moderate Anxiety level (45-59); Marked to Severe Anxiety level (6074); Extreme Anxiety level (75-80) ${ }^{[24]}$.

iv.ZSDS: Zung Self-Rating Depression scale quality life questionnaire is also a 20 items short selfadministered survey that was designed by William W. K. Zung to assess the level of four common characteristics of depression for patients: the pervasive effect, the physiological equivalents, other disturbances, and psychomotor activities. There were ten positively worded and ten negatively worded questions. Each question was scored on a scale of 1-4 (a little of the time, some of the time, good part of the time, most of the time) ${ }^{[25]}$. The higher scores indicated severe depression level. The raw scores were counted up and multiplied by 1.25 to reach a standardized score, according to the instructions that accompanied the scale $e^{[25]}$. The ZSDS Index score followed the crierion: Normal Range $(<50)$; Mild Depression level $(<60)$; Moderate Depression level (<70); Extreme Depression level $(>70){ }^{[25]}$.

\section{(b) Statistical analyses}

The database and statistical analysis was performed by using SPSS v.17 software. The independent variables analyzed were Socio-demographic characteristics (sex/gender, age, education level, marital status, employment type, income level (per annum), job background, local residence), Clinical characteristics (smoking habit, drinking habit, tobacco use, health insurance, tumor type \& location, 
metastasis involved, corresponding cycle number for both chemotherapy and radiotherapy treatment); Anxiety and Depression of the patients as assessed by ZSAS and ZSDS respectively. The dependent variables included: subscale and overall QOL scores, and Health satisfaction as measured by WHOQOL-Bref Questionnaire (Tool/instrument). Descriptive statistics computation techniques were applied to the discrete and continuous data. Measures such as mean, standard deviation, minimum and maximum range were developed from the continuous data. Relative frequency was calculated for discrete data. Mean with Standard deviation (SD) was used to summarize the age of patients. Chisquare test was performed to assess the effect of different sociodemographic factors and clinical characteristics on the QOL of the cancer patients. Student's $t$-test was used to compare sample means for study variables (anxiety, depression and QOL). Bivariate analysis was performed to assess the predictors of QOL. Based on the survey/ research, Pearson Correlation coefficient test denoted by $r$ was calculatedto assess the particular characteristic symptomatic function of anxiety and depression instrument that significantly affected the QOL domains, i.e., either positively or negatively. Paired ttest was used to compare difference between score means of different domains. A $p$-value $<0.05$ was considered as statistical significant.

\section{Socio-demographic characteristics of the patients}

Table 4.1. Socio-demographic characteristics in the form of Frequency and percentage of variables of the patients and correlation in the two groups, i.e., Chemotherapy and Radiotherapy

\begin{tabular}{|c|c|c|c|c|c|c|}
\hline \multirow[t]{2}{*}{ S.No. } & \multirow[t]{2}{*}{ Variables } & \multirow[t]{2}{*}{ Parameters } & \multicolumn{2}{|c|}{ Chemotherapy } & \multicolumn{2}{|c|}{ Radiotherapy } \\
\hline & & & $\begin{array}{l}\text { Frequency } \\
\text { (n) }\end{array}$ & $\begin{array}{l}\text { Percentage } \\
\text { (\%) }\end{array}$ & $\begin{array}{l}\text { Frequenc } \\
\text { y (n) }\end{array}$ & $\begin{array}{l}\text { Percentage } \\
(\%)\end{array}$ \\
\hline 1. & Gender & $\begin{array}{l}\text { a. Male } \\
\text { b. Female }\end{array}$ & $\begin{array}{l}6 \\
24 \\
\end{array}$ & $\begin{array}{l}20.0 \% \\
80.0 \% \\
\end{array}$ & $\begin{array}{l}15 \\
15\end{array}$ & $\begin{array}{l}50.0 \% \\
50.0 \%\end{array}$ \\
\hline 2. & Age & $\begin{array}{l}\text { a. } 18-30 \text { years } \\
\text { b. } 30 \\
\text { c. } 46 \\
\text { d. } 60\end{array}$ & $\begin{array}{l}5 \\
17 \\
8\end{array}$ & $\begin{array}{l}16.7 \% \\
56.7 \% \\
26.7 \%\end{array}$ & $\begin{array}{l}6 \\
15 \\
9\end{array}$ & $\begin{array}{l}20.0 \% \\
50.0 \% \\
30.0 \%\end{array}$ \\
\hline 3. & $\begin{array}{l}\text { Marital } \\
\text { status }\end{array}$ & $\begin{array}{l}\text { a. Unmarried } \\
\text { b. Married } \\
\text { c. Widow } \\
\text { d. Divorced/ } \\
\text { Legally separated } \\
\text { e. Others }\end{array}$ & $\begin{array}{l}30 \\
30\end{array}$ & $100.0 \%$ & $\begin{array}{l}1 \\
28 \\
1\end{array}$ & $\begin{array}{l}3.3 \% \\
93.3 \% \\
3.3 \%\end{array}$ \\
\hline 4. & $\begin{array}{l}\text { Educational } \\
\text { status }\end{array}$ & $\begin{array}{l}\text { a. Illiterate } \\
\text { b. Literate } \\
\text { i. Primary } \\
\text { ii. Secondary } \\
\text { iii. Tertiary }\end{array}$ & $\begin{array}{l}4 \\
10 \\
15\end{array}$ & $\begin{array}{l}3.3 \% \\
\\
13.3 \% \\
33.3 \% \\
50.0 \%\end{array}$ & $\begin{array}{l}5 \\
11 \\
12\end{array}$ & $\begin{array}{l}6.7 \% \\
16.7 \% \\
36.7 \% \\
40.0 \% \\
\end{array}$ \\
\hline 5. & Occupation & $\begin{array}{l}\text { a. Service } \\
\text { b. Business } \\
\text { c. Housewife }\end{array}$ & $\begin{array}{l}3 \\
3 \\
21\end{array}$ & $\begin{array}{l}10.0 \% \\
10.0 \% \\
70.0 \%\end{array}$ & $\begin{array}{l}10 \\
2 \\
11\end{array}$ & $\begin{array}{l}33.3 \% \\
6.7 \% \\
36.7 \%\end{array}$ \\
\hline
\end{tabular}


Texila International Journal of Clinical Research

Volume 3, Issue 2, Dec 2016

\begin{tabular}{|c|c|c|c|c|c|c|}
\hline & & $\begin{array}{l}\text { d. Freelancers } \\
\text { e. Pensioners } \\
\text { f. Domestic duties } \\
\text { g. Cultivation }\end{array}$ & 3 & $10.0 \%$ & $\begin{array}{l}1 \\
4 \\
1 \\
1\end{array}$ & $\begin{array}{l}3.3 \% \\
13.3 \% \\
3.3 \% \\
3.3 \%\end{array}$ \\
\hline 6. & $\begin{array}{l}\text { Type of } \\
\text { family }\end{array}$ & $\begin{array}{l}\text { a. Nuclear } \\
\text { b. Joint }\end{array}$ & $\begin{array}{l}16 \\
14\end{array}$ & $\begin{array}{l}53.3 \% \\
46.7 \%\end{array}$ & $\begin{array}{l}9 \\
21\end{array}$ & $\begin{array}{l}30.0 \% \\
70.0 \%\end{array}$ \\
\hline 7. & Cohabitants & $\begin{array}{l}\text { a. Living alone } \\
\text { b. Living with } \\
\text { partner } \\
\text { c. Living with } \\
\text { partner and } \\
\text { children } \\
\text { d. Living with } \\
\text { children }\end{array}$ & $\begin{array}{l}15 \\
1 \\
14\end{array}$ & $\begin{array}{l}50.0 \% \\
3.3 \% \\
46.7 \%\end{array}$ & $\begin{array}{l}4 \\
5 \\
21\end{array}$ & $\begin{array}{l}13.3 \% \\
16.67 \% \\
70.0 \%\end{array}$ \\
\hline 8. & $\begin{array}{l}\text { Annual } \\
\text { income }\end{array}$ & $\begin{array}{l}\text { a. NA } \\
\text { b. } \leq 20 \\
\text { c. } 20 \\
\text { d. } 30 \\
\text { e. } 41 \\
\text { f. } 84\end{array}$ & $\begin{array}{l}22 \\
8\end{array}$ & $\begin{array}{l}73.3 \% \\
26.7 \% \\
\end{array}$ & $\begin{array}{l}11 \\
19\end{array}$ & $\begin{array}{l}36.7 \% \\
63.3 \%\end{array}$ \\
\hline 9. & $\begin{array}{l}\text { Place of } \\
\text { residence }\end{array}$ & $\begin{array}{l}\text { a. Small town } \\
\text { b. Big town }\end{array}$ & $\begin{array}{l}1 \\
29 \\
\end{array}$ & $\begin{array}{l}3.3 \% \\
96.7 \% \\
\end{array}$ & \begin{tabular}{|l|}
8 \\
22 \\
\end{tabular} & $\begin{array}{l}26.7 \% \\
73.3 \% \\
\end{array}$ \\
\hline
\end{tabular}

The mean age (SD) of the patients in Chemotherapy group: 54.37 (11.08) [Range: 32-75]. The mean age (SD) of the patients in Radiotherapy group: 54.21 (11.07) [Range: 32-75].

(All tests were performed using Pearson $\chi 2$ test for association analysis)

\section{Clinical characteristics of the patients}

Table 4.2. Clinicalcharacteristics in the form of Frequency and percentage of variables of the

\begin{tabular}{|c|c|c|c|c|c|c|}
\hline \multirow[t]{2}{*}{ S.No. } & \multirow[t]{2}{*}{ Variables } & \multirow[t]{2}{*}{ Parameters } & \multicolumn{2}{|c|}{ Chemotherapy } & \multicolumn{2}{|c|}{ Radiotherapy } \\
\hline & & & $\begin{array}{l}\text { Frequency } \\
\text { (n) }\end{array}$ & $\begin{array}{l}\text { Percentage } \\
\text { (\%) }\end{array}$ & $\begin{array}{l}\text { Frequency } \\
\text { (n) }\end{array}$ & $\begin{array}{l}\text { Percentage } \\
(\%)\end{array}$ \\
\hline 1. & Smoking habit & $\begin{array}{l}\text { a. Non-smoker } \\
\text { b. Ex-smoker }\end{array}$ & $\begin{array}{l}27 \\
3\end{array}$ & $\begin{array}{l}90.0 \% \\
10.0 \%\end{array}$ & $\begin{array}{l}27 \\
4\end{array}$ & $\begin{array}{l}90.0 \% \\
10.0 \%\end{array}$ \\
\hline 2. & Drinking habit & $\begin{array}{l}\text { a. Non-drinker } \\
\text { b. Ex-drinker }\end{array}$ & 30 & $100.0 \%$ & $\begin{array}{l}26 \\
4 \\
\end{array}$ & $\begin{array}{l}86.7 \% \\
13.3 \% \\
\end{array}$ \\
\hline 3. & Tobacco use & $\begin{array}{l}\text { a. Yes } \\
\text { b. No }\end{array}$ & $\begin{array}{l}1 \\
29\end{array}$ & $\begin{array}{l}3.3 \% \\
96.7 \% \\
\end{array}$ & $\begin{array}{l}7 \\
23 \\
\end{array}$ & $\begin{array}{l}23.3 \% \\
76.7 \% \\
\end{array}$ \\
\hline 4. & $\begin{array}{l}\text { Health } \\
\text { insurance }\end{array}$ & $\begin{array}{l}\text { a. Yes } \\
\text { b. No }\end{array}$ & $\begin{array}{l}23 \\
7 \\
\end{array}$ & $\begin{array}{l}76.7 \% \\
23.3 \% \\
\end{array}$ & $\begin{array}{l}19 \\
11 \\
\end{array}$ & $\begin{array}{l}63.3 \% \\
36.7 \% \\
\end{array}$ \\
\hline 5. & $\begin{array}{l}\text { Type of health } \\
\text { insurance }\end{array}$ & $\begin{array}{l}\text { a. Government } \\
\text { medically } \\
\text { insured }\end{array}$ & 10 & $33.3 \%$ & 12 & $40.0 \%$ \\
\hline
\end{tabular}


Texila International Journal of Clinical Research

Volume 3, Issue 2, Dec 2016

\begin{tabular}{|c|c|c|c|c|c|c|}
\hline & & $\begin{array}{l}\text { b. TPA } \\
\text { c. Cash }\end{array}$ & $\begin{array}{l}13 \\
7\end{array}$ & $\begin{array}{l}43.3 \% \\
23.3 \%\end{array}$ & $\begin{array}{l}7 \\
11\end{array}$ & $\begin{array}{l}23.3 \% \\
36.7 \%\end{array}$ \\
\hline 6. & $\begin{array}{l}\text { Support by } \\
\text { NGO }\end{array}$ & $\begin{array}{l}\text { a. Yes } \\
\text { b. No }\end{array}$ & 30 & $100.0 \%$ & $\begin{array}{l}0 \\
30\end{array}$ & $100 \%$ \\
\hline 7. & $\begin{array}{l}\text { Cancer tumor } \\
\text { location }\end{array}$ & $\begin{array}{l}\text { a. Ca Breast } \\
\text { b. Ca Head } \\
\text { c. Ca Neck }\end{array}$ & $\begin{array}{l}23 \\
1 \\
6\end{array}$ & $\begin{array}{l}76.7 \% \\
3.3 \% \\
20.0 \%\end{array}$ & $\begin{array}{l}10 \\
5 \\
15\end{array}$ & $\begin{array}{l}33.3 \% \\
16.7 \% \\
50.0 \%\end{array}$ \\
\hline 8. & $\begin{array}{l}\text { Disease } \\
\text { acceptance }\end{array}$ & $\begin{array}{l}\text { a. Yes } \\
\text { b. No }\end{array}$ & $\begin{array}{l}23 \\
7\end{array}$ & $\begin{array}{l}76.7 \% \\
23.3 \% \\
\end{array}$ & $\begin{array}{l}15 \\
15 \\
\end{array}$ & $\begin{array}{l}50.0 \% \\
50.0 \%\end{array}$ \\
\hline 9. & $\begin{array}{l}\text { Reproductive } \\
\text { age of women }\end{array}$ & $\begin{array}{l}\text { a. Pre-menopausal } \\
\text { b. Menopausal }\end{array}$ & $\begin{array}{l}10 \\
14\end{array}$ & $\begin{array}{l}33.3 \% \\
46.7 \% \\
\end{array}$ & $\begin{array}{l}9 \\
6 \\
\end{array}$ & $\begin{array}{l}30.0 \% \\
20.0 \% \\
\end{array}$ \\
\hline 10. & Cancer type & $\begin{array}{l}\text { a. Primary cancer } \\
\text { b. Recurrent } \\
\text { cancer }\end{array}$ & 30 & $100.0 \%$ & 30 & $100.0 \%$ \\
\hline 11. & $\begin{array}{l}\text { Co-existence } \\
\text { of metastasis }\end{array}$ & $\begin{array}{l}\text { a. Yes } \\
\text { b. No }\end{array}$ & $\begin{array}{l}8 \\
22\end{array}$ & $\begin{array}{l}26.7 \% \\
73.4 \%\end{array}$ & 30 & $100.0 \%$ \\
\hline 12. & $\begin{array}{l}\text { Chemotherapy } \\
\text { 's cycle during } \\
\text { the interview } \\
\text { of QLQ }\end{array}$ & $\begin{array}{l}\text { a. } 1^{\text {st }} \\
\text { b. } 2^{\text {nd }} \\
\text { c. } 3^{\text {rd }} \\
\text { d. } 4^{\text {th }} \\
\text { e. } 5^{\text {th }} \\
\text { f. } 6^{\text {th }}\end{array}$ & $\begin{array}{l}2 \\
15 \\
6 \\
6 \\
1\end{array}$ & $\begin{array}{l}6.7 \% \\
50.0 \% \\
20.0 \% \\
20.0 \% \\
3.3 \% \\
\end{array}$ & & \\
\hline 13 & $\begin{array}{l}\text { Radiotherapy' } \\
\text { s cycle during } \\
\text { the interview } \\
\text { of QLQ }\end{array}$ & $\begin{array}{l}\text { a. } 10^{\text {th }}-15^{\text {th }} \\
\text { b. } 16^{\text {th }}-20^{\text {th }} \\
\text { c. } 21^{\text {st }}-25^{\text {th }} \\
\text { d. } 26^{\text {th }} 30^{\text {th }}\end{array}$ & & & $\begin{array}{l}21 \\
3 \\
1\end{array}$ & $\begin{array}{l}16.7 \% \\
\\
70.0 \% \\
10.0 \% \\
3.3 \%\end{array}$ \\
\hline
\end{tabular}

patients and correlation in the two groups, i.e., Chemotherapy or Radiotherapy

(All tests were performed using Pearson $\chi^{2}$ test for association analysis).

From the above table no. 4.1 and 4.2, it was observed that there was no significant correlation and association between the QOL and variables such as age, sex, marital status, and occupational function, etc. Furthermore, no correlation was found between QOL and the patients' educational level (literate 
Texila International Journal of Clinical Research

Volume 3, Issue 2, Dec 2016

or illiterate). The age distribution indicated that the adult and elderly people were the most affected. Hence, no significant correlation of the Socio-demographic and clinical characteristics of the patients (sample) with the domains of quality of life was observed.

Table 4.3. Internal consistency of WHOQOL-Bref, ZSAS, and ZSDS domains as measured by Cronbach's $\alpha$ based on participants who completed the Questionnaire during Chemotherapy or Radiotherapy sessions.

\begin{tabular}{|c|c|c|c|c|c|c|c|}
\hline Measure & Purpose & Domains & Scale & $\begin{array}{l}\text { Time } \\
\text { frame }\end{array}$ & $\begin{array}{l}\text { No. } \\
\text { of } \\
\text { items }\end{array}$ & $\begin{array}{l}\text { Administer } \\
\text { ed by and } \\
\text { (completio } \\
\text { n time) }\end{array}$ & Reliability \\
\hline $\begin{array}{l}\text { World } \\
\text { Health } \\
\text { Organizatio } \\
\text { n Quality } \\
\text { of Life - } \\
\text { Brief } \\
\text { Version } \\
\text { (WHOQO } \\
\text { L-BREF) } \\
\text { [23] }\end{array}$ & $\begin{array}{l}\text { Designed to } \\
\text { examine } \\
\text { domain level } \\
\text { profiles } \\
\text { assessing } \\
\text { quality of } \\
\text { life }^{[23]} \text {. }\end{array}$ & $\begin{array}{l}\text { Four domains: } \\
\text { Physical } \\
\text { health; } \\
\text { psychological; } \\
\text { social } \\
\text { relationships; } \\
\text { environment }{ }^{[23} \\
\text { ]. }\end{array}$ & $\begin{array}{l}\text { Five point } \\
\text { Likert scale } \\
\text { with varying } \\
\text { anchors }^{[23]} \text {. }\end{array}$ & $\begin{array}{l}\text { Past } \\
\text { two } \\
\text { weeks } \\
\text { 23]. }\end{array}$ & 26 & $\begin{array}{l}\text { Self- } \\
\text { administere } \\
\text { d } \\
\text { (estimated } \\
15-20 \\
\text { minutes) }^{[23]} \\
\text {. }\end{array}$ & 0.672 \\
\hline $\begin{array}{l}\text { Zung self- } \\
\text { rating } \\
\text { anxiety } \\
\text { scale } \\
{[24] \text {. }}\end{array}$ & $\begin{array}{l}\text { Designed to } \\
\text { measure } \\
\text { anxiety }^{[24]} \text {. }\end{array}$ & Anxiety $^{[24]}$. & $\begin{array}{l}\text { Four point } \\
\text { Likert scale } \\
\text { ranging } \\
\text { from } 1 \text { (A } \\
\text { little of the } \\
\text { time) to } 4 \\
\text { (Most of the } \\
\text { time) }^{[24]} \text {. }\end{array}$ & $\begin{array}{l}\text { Last } 5 \\
\text { days }{ }^{[24} \\
\text { ]. }\end{array}$ & 20 & $\begin{array}{l}\text { Self- } \\
\text { administere } \\
d \\
\text { (estimated } \\
10 \\
\text { minutes) }^{[24]} \\
\text {. }\end{array}$ & 0.742 \\
\hline $\begin{array}{l}\text { Zung self- } \\
\text { rating } \\
\text { depression } \\
\text { scale }^{[25]} \text {. }\end{array}$ & $\begin{array}{l}\text { Designed to } \\
\text { measure } \\
\text { depression }^{[25]} \text {. }\end{array}$ & Depression $^{[25]}$. & $\begin{array}{l}\text { Four point } \\
\text { Likert scale } \\
\text { ranging } \\
\text { from } 1 \text { (A } \\
\text { little of the } \\
\text { time) to } 4 \\
\text { (Most of the } \\
\text { time) }^{[25]} \text {. }\end{array}$ & $\begin{array}{l}\text { Last } 5 \\
\text { days }{ }^{[25} \\
\text { ]. }\end{array}$ & 20 & $\begin{array}{l}\text { Self- } \\
\text { administere } \\
d \\
\text { (estimated } \\
10 \\
\text { minutes) }^{[25]} \\
\text {. }\end{array}$ & 0.532 \\
\hline
\end{tabular}

Reliability of the scales and questionnaire (TOOLS/ INSTRUMENTS) used in the study

12(40\%) of patients filled the English version of QLQ whereas 18(60\%) of patients filled the translated vernacular version (Hindi language) in Chemotherapy section. On the other hand, 8(26.6\%) of patients filled the English version of QLQ whereas 22(73.3\%) of patients filled the translated vernacular version (Hindi language) in Radiotherapy section. 


\section{Impact of anxiety and depression on different domains of qol in chemotherapy group}

Table 4.4. Paired $t$-test between WHOQOL-Bref domains, ZSAS, and ZSDS for Chemotherapy $(\mathrm{n}=$ 30)\&Radiotherapy $(n=30)$

\begin{tabular}{|c|c|c|c|c|c|c|c|c|}
\hline \multicolumn{9}{|c|}{ CHEMOTHERAPY } \\
\hline & \multicolumn{4}{|c|}{ Paired differences } & \multirow[t]{3}{*}{$t$-test } & \multirow[t]{3}{*}{ r- value } & \multirow[t]{3}{*}{$\mathrm{df}$} & \multirow{3}{*}{\begin{tabular}{|l|} 
Sig. \\
$(2-$ \\
tailed $)$
\end{tabular}} \\
\hline & \multirow[t]{2}{*}{ Mean } & \multirow[t]{2}{*}{ SD } & \multicolumn{2}{|c|}{ 95\%CI of the difference } & & & & \\
\hline & & & Lower & Upper & & & & \\
\hline $\begin{array}{l}\text { Pair } 1 \\
\text { (ZSAS CT- } \\
\text { DOM1 CT) }\end{array}$ & 26.95 & 19.13 & 19.81 & 34.10 & 7.71 & $\begin{array}{l}r=- \\
0.480 \\
p=0.007\end{array}$ & 29 & $\mathrm{p}<0.001$ \\
\hline $\begin{array}{l}\text { Pair } 2 \\
\text { (ZSAS CT- } \\
\text { DOM4 CT) }\end{array}$ & 11.62 & 14.63 & 6.15 & 17.09 & 4.35 & $\begin{array}{l}r=- \\
0.384 \\
p=0.036\end{array}$ & 29 & $\mathrm{p}<0.001$ \\
\hline $\begin{array}{l}\text { Pair } 3 \\
\text { (ZSDS CT- } \\
\text { DOM3 CT) }\end{array}$ & 17.00 & 12.79 & 12.22 & 21.77 & 7.27 & $\begin{array}{l}\mathrm{r}=- \\
0.371 \\
\mathrm{p}=0.043\end{array}$ & 29 & $\mathrm{p}<0.001$ \\
\hline \multicolumn{9}{|c|}{ RADIOTHERAPY } \\
\hline $\begin{array}{l}\text { Pair } 1 \\
\text { (ZSAS RT- } \\
\text { DOM3 RT) } \\
\end{array}$ & 13.22 & 17.78 & 6.58 & 19.86 & 4.07 & $\begin{array}{l}\mathrm{r}=- \\
0.435 \\
\mathrm{p}=0.016\end{array}$ & 29 & $\mathrm{p}<0.001$ \\
\hline $\begin{array}{l}\text { Pair } 2 \\
\text { (ZSDS RT- } \\
\text { DOM3 RT) }\end{array}$ & 11.39 & 15.85 & 5.47 & 17.31 & 3.93 & $\begin{array}{l}r=- \\
0.453 \\
p=0.012\end{array}$ & 29 & $\mathrm{p}<0.001$ \\
\hline
\end{tabular}

It could be observed from the above table that in chemotherapy group, anxiety level affected the Physical health domain ( $r=-0.480 ; \mathrm{p}=0.007)$, and Environmental domain $(\mathrm{r}=-0.384 ; \mathrm{p}=0.036)$. On the other hand, depression level affected the social relationship domain $(\mathrm{r}=-0.371 ; \mathrm{p}=0.043)$. These results were found to be statistically significant at $\mathrm{p}<0.05$ (critical value). Moreover, the relationship between the anxiety and QOL domains, and depression and QOL domains was found to be negatively correlated which signified that there was a marked decrease in QOL due to increase in anxiety or depression level of the cancer patients. On the other hand, in radiotherapy group, anxiety level affected the Social relationship domain $(\mathrm{r}=-0.435$; $\mathrm{p}=0.016)$, and depression level affected the social relationship domain $(\mathrm{r}=-0.453 ; \mathrm{p}=0.012$ ). These results were found to be statistically significant at $\mathrm{p}$ $<0.05$ (critical value). Moreover, the relationship between the anxiety and QOL domains, and depression and QOL domains was found to be negatively correlated which signified that that there was a marked decrease in QOL due to increase in anxiety or depression level of the cancer patients. 
Facets with greater influence of Anxiety and Depression in each domain of WHOQOLBref of patients undergoing Chemotherapy treatment $(n=30)$ \&Radiotherapy treatment $(\mathbf{n}=30)$ (Pearson Correlation test)

Table 4.5: Anxiety and depression affecting the Q.no. (facets) in particular domain of WHOQOL-Bref in chemotherapy group $(n=30)$ \& radiotherapy group $(n=30)$

\begin{tabular}{|c|c|c|c|}
\hline \multicolumn{4}{|c|}{ CHEMOTHERAPY } \\
\hline \multicolumn{4}{|c|}{ ANXIETY x WHOQOL Bref Question } \\
\hline $\begin{array}{l}\text { Question } \\
\text { no. }\end{array}$ & Facet evaluated & $\mathrm{r}$ & $\mathrm{p}$ \\
\hline Q 8. & Activities of daily living & -0.483 & 0.007 \\
\hline Q 9. & Work Capacity & -0.606 & $\mathrm{p}<0.001$ \\
\hline Q 17. & Social support & -0.521 & 0.003 \\
\hline Q 19. & Security & -0.376 & 0.041 \\
\hline Q 23. & Leisure activity\& Recreation & -0.519 & 0.003 \\
\hline \multicolumn{4}{|c|}{ DEPRESSION x WHOQOL Bref Question } \\
\hline Q 17. & Social support & -0.438 & 0.016 \\
\hline \multicolumn{4}{|c|}{ RADIOTHERAPY } \\
\hline \multicolumn{4}{|c|}{ ANXIETY x WHOQOL Bref Question } \\
\hline Q 16. & $\begin{array}{l}\text { Personal relations/ Family } \\
\text { happiness }\end{array}$ & -0.541 & 0.002 \\
\hline \multicolumn{4}{|c|}{ DEPRESSION x WHOQOL Bref Question } \\
\hline Q 16. & $\begin{array}{l}\text { Personal relations/ Family } \\
\text { happiness }\end{array}$ & -0.593 & 0.001 \\
\hline
\end{tabular}

In the Chemotherapy group, Anxiety affected: the activities of daily living and Working capacity in the Physical health domain; and sense of security and leisure activity and recreation in Environmental domain with $\mathrm{p}<0.05$. On the other hand, in the Chemotherapy group, Depression affected the social support in social relationship domain with $\mathrm{p}<0.05$. In the Radiotherapy group, Anxiety affected the personal relations in social relationship domain with $\mathrm{p}<0.05$. On the other hand, in the Radiotherapy group, Depression affected the personal relations in social relationship domain with $\mathrm{p}<0.05$.

QOL domain scores for chemotherapy sessions $(n=30)$

Table 4.6: QOL domain scores for Chemotherapy sessions $(\mathrm{n}=30)$

\begin{tabular}{|l|l|l|l|l|l|}
\hline $\begin{array}{l}\text { Domains for } \\
\text { QOL/Scale }\end{array}$ & $\begin{array}{l}\text { Minimum } \\
\text { possible } \\
\text { raw score }\end{array}$ & $\begin{array}{l}\text { Maximum } \\
\text { possible } \\
\text { raw score }\end{array}$ & $\begin{array}{l}\text { Mean of } \\
\text { raw } \\
\text { score }\end{array}$ & $\begin{array}{l}\text { SD of } \\
\text { raw } \\
\text { score }\end{array}$ & $\begin{array}{l}\text { Mean of Score } \\
\text { translated on a } \\
\text { scale of 100 }\end{array}$ \\
\hline Physical & 7 & 35 & 17.97 & 3.09 & 39.66 \\
\hline Psychological & 6 & 30 & 17.94 & 1.87 & 49.76 \\
\hline Social & 3 & 15 & 7.77 & 0.89 & 41.66 \\
\hline Environmental & 8 & 40 & 24.83 & 1.94 & 55.00 \\
\hline ZSAS Scores & 20 & 80 & 53.30 & 9.10 & 66.62 \\
\hline ZSDS Scores & 20 & 80 & 46.94 & 5.63 & 58.66 \\
\hline
\end{tabular}

In the chemotherapy group, the physical domain was the most affected domain, followed by social domain, psychological domain and environmental domain in the descending order. 
QOL domain scores for Radiotherapy sessions $(n=30)$

Table 4.7. QOL domain scores for radiotherapy sessions $(n=30)$

\begin{tabular}{|l|l|l|l|l|l|}
\hline $\begin{array}{l}\text { Domains for } \\
\text { QOL/Scale }\end{array}$ & $\begin{array}{l}\text { Minimum } \\
\text { possible raw } \\
\text { score }\end{array}$ & $\begin{array}{l}\text { Maximum } \\
\text { possible raw } \\
\text { score }\end{array}$ & $\begin{array}{l}\text { Mean of } \\
\text { raw score }\end{array}$ & $\begin{array}{l}\text { SD of raw } \\
\text { score }\end{array}$ & $\begin{array}{l}\text { Mean of Score } \\
\text { translated on a } \\
\text { scale of 100 }\end{array}$ \\
\hline Physical & 7 & 35 & 17.37 & 4.18 & 37.13 \\
\hline Psychological & 6 & 30 & 17.74 & 2.78 & 49.10 \\
\hline Social & 3 & 15 & 7.77 & 1.25 & 40.73 \\
\hline Environmental & 8 & 40 & 25.10 & 2.35 & 55.26 \\
\hline ZSAS Scores & 20 & 80 & 43.17 & 8.04 & 53.95 \\
\hline ZSDS Scores & 20 & 80 & 41.70 & 6.05 & 52.12 \\
\hline
\end{tabular}

In the Radiotherapy group, the physical domain was the most affected domain, followed by social domain, psychological domain and environmental domain in the descending order.

From the Table 4.6 and Table 4.7, it can be elucidated that anxiety and depression scores were more high in the Chemotherapy group as compared to the Radiotherapy group that indicated that the Chemotherapy group had higher levels of Anxiety and Depression during Chemotherapy or Radiotherapy treatment.

Facets/Items of Anxiety domain in ZSAS and Depression domain in ZSDS affecting WHOQOLBref domains in Chemotherapy group $(n=30)$

Table 4.8. Symptoms of anxiety and depression affecting particular domains of WHOQOL-Bref in chemotherapy group $(\mathrm{n}=30)$

\begin{tabular}{|c|c|c|c|c|c|}
\hline & WHOQOL Bref Questi & n x ANXI & (ZSAS) & & \\
\hline & $\begin{array}{l}\text { WHOQOL-Bref } \\
\text { Domain }\end{array}$ & $\begin{array}{l}\text { ZSAS } \\
\text { Question } \\
\text { no. }\end{array}$ & $\begin{array}{l}\text { Facet } \\
\text { evaluated/Symptoms }\end{array}$ & $\mathbf{r}$ & $\mathbf{p}$ \\
\hline & PHYSICAL DOMAIN & Q 1. & More nervousness & -0.382 & 0.032 \\
\hline & 1. Activities of daily & Q 10. & Fast heart beat & -0.445 & 0.014 \\
\hline & living & Q 11. & Dizzy spells affect & -0.450 & 0.013 \\
\hline & & Q 12. & Fainting spells & -0.487 & 0.006 \\
\hline $\mathbf{C}$ & & Q 17. & Dry and warm hands & -0.548 & 0.002 \\
\hline $\mathbf{H}$ & & Q 20. & Nightmare problem & -0.466 & 0.010 \\
\hline $\mathbf{E}$ & 2. Work capacity & Q 3. & Upset & -0.380 & 0.039 \\
\hline $\mathbf{M}$ & & Q 4. & Falling down feeling & -0.481 & 0.007 \\
\hline $\mathbf{O}$ & & Q 5. & Positive feeling & 0.470 & 0.009 \\
\hline $\mathbf{T}$ & & Q 9. & Calmness & 0.487 & 0.006 \\
\hline $\begin{array}{l}\mathbf{H} \\
\mathbf{F}\end{array}$ & & Q 11. & Dizzy spells affect & -0.656 & $\mathbf{p}<0.001$ \\
\hline $\begin{array}{l}\mathbf{E} \\
\mathbf{R}\end{array}$ & & Q 12. & Fainting spells & -0.634 & $\mathrm{p}<0.001$ \\
\hline A & & Q 17. & Dry and warm hands & -0.483 & 0.007 \\
\hline $\mathbf{P}$ & & Q 20. & Nightmare problem & -0.623 & $\mathbf{p}<0.001$ \\
\hline $\mathbf{Y}$ & ENVIRONMENTAL & Q 5. & Positive feeling & 0.418 & 0.021 \\
\hline & $\begin{array}{l}\text { DOMAIN } \\
\text { 3. Security }\end{array}$ & Q 6. & $\begin{array}{l}\text { Shaking of arms and } \\
\text { legs }\end{array}$ & -0.383 & 0.037 \\
\hline & & Q 9. & Calmness & 0.436 & 0.016 \\
\hline & & Q 10. & Fast heart beat & -0.397 & 0.030 \\
\hline
\end{tabular}




\begin{tabular}{|c|c|c|c|c|}
\hline & Q 11. & Dizzy spells affect & -0.366 & 0.046 \\
\hline & Q 12. & Fainting spells & -0.376 & 0.041 \\
\hline & Q 20. & Nightmare problem & -0.484 & 0.007 \\
\hline \multirow[t]{7}{*}{ 4. Leisure Activity } & Q 1. & More nervousness & -0.424 & 0.020 \\
\hline & Q 5. & Positive feeling & 0.504 & 0.004 \\
\hline & Q 9. & Calmness & 0.436 & 0.016 \\
\hline & Q 10. & Fast heart beat & -0.550 & 0.002 \\
\hline & Q 11. & Dizzy spells affect & -0.397 & 0.030 \\
\hline & Q 12. & Fainting spells & -0.401 & 0.028 \\
\hline & Q 20. & Nightmare problem & -0.575 & 0.001 \\
\hline \multicolumn{5}{|c|}{ WHOQOL Bref Question x DEPRESSION (ZSDS) } \\
\hline $\begin{array}{l}\text { SOCIAL DOMAIN } \\
1 . \quad \text { Social Support } \\
1 . \quad\end{array}$ & Q 9. & Tachycardia & -0.431 & 0.018 \\
\hline
\end{tabular}

From the above table, it could be depicted that Anxiety symptoms such as, Nervousness, fast heartbeat, dizzy \& fainting spells affect, dry warm hands, and nighmares with $p<0.05$ was significantly correlated with the Activities of the daily living in the Physical domain of the chemotherapy group. Moreover, Anxiety symptoms such as, upset feeling, falling down feeling, positive feeling, calmness, dizzy \& fainting spells affect dry warm hands, and nighmares with $\mathrm{p}<$ 0.05 was significantly correlated with the work capacity in the Physical domain of the chemotherapy group. In the environmental domain, the WHOQOL-Bref factor namely, Security and Leisure activity was significantly affected by Anxiety symptoms such as, Positive feeling, Shaking of arms and legs, Calmness, Fast heart beat, Dizzy spells affect, Fainting spells, Nightmare problem; and More nervousness, Positive feeling, Calmness, Fast heart beat, Dizzy spells affect, Fainting spells, Nightmare problem, respectively with $\mathrm{p}<0.05$. On the other hand, Depression symptom such as, Tachycardia affected Social support of social domain in the Chemotherapy group that was significantly correlated with $\mathrm{p}<0.05$. In chemotherapy group, the physical domain consisting of Activities of daily living and Work capacity were significantly strongly correlated to each other ( $\mathrm{r}=$ 0.742; $\mathrm{p}<0.001$ ). On the other hand, the Environmental domain that consisted of Security and Leisure activity factor were significantly correlated to each other $(r=0.567 ; p=0.001)$ in the chemotherapy group. 


\section{Facets/Items of Anxiety domain in ZSAS and Depression domain in ZSDS affecting WHOQOL-Bref domains in Radiotherapy group $(n=30)$}

Table 4.9: Symptoms of anxiety and depression affecting particular domains of WHOQOL-Bref in radiotherapy group $(\mathrm{n}=30)$

\begin{tabular}{|c|c|c|c|c|c|}
\hline \multirow{2}{*}{$\begin{array}{l}\mathbf{R} \\
\mathbf{A}\end{array}$} & \multicolumn{5}{|c|}{ WHOQOL Bref Question x ANXIETY (ZSAS) } \\
\hline & $\begin{array}{l}\text { WHOQOL-Bref } \\
\text { Domain }\end{array}$ & $\begin{array}{l}\text { ZSAS Question } \\
\text { no. }\end{array}$ & $\begin{array}{l}\text { Facet } \\
\text { evaluated/Symptoms }\end{array}$ & $\mathbf{r}$ & $\mathbf{p}$ \\
\hline $\begin{array}{l}\text { A } \\
\text { D }\end{array}$ & \multirow{4}{*}{$\begin{array}{l}\text { SOCIAL DOMAIN } \\
\text { 1. Personal } \\
\text { relationship }\end{array}$} & Q 7. & $\begin{array}{l}\text { Headache, neck, back } \\
\text { pain }\end{array}$ & -0.409 & 0.025 \\
\hline $\mathbf{1}$ & & Q 8. & Weakness & -0.454 & 0.012 \\
\hline $\mathbf{T}$ & & Q 10. & Fast heart beat & -0.387 & 0.034 \\
\hline $\mathbf{H}$ & & Q 18. & Hot and blushing face & -0.372 & $\mathbf{0 . 0 4 3}$ \\
\hline $\mathbf{E}$ & \multicolumn{5}{|c|}{ WHOQOL Bref Question x DEPRESSION (ZSDS) } \\
\hline $\mathbf{R}$ & \multirow{5}{*}{$\begin{array}{l}\text { SOCIAL DOMAIN } \\
\text { 2. Personal } \\
\text { relationship }\end{array}$} & Q 1. & Depressed affect & -0.394 & 0.031 \\
\hline A & & Q 9. & Tachycardia & -0.387 & 0.035 \\
\hline $\mathbf{P}$ & & Q 10. & Fatigue & -0.417 & 0.022 \\
\hline $\mathbf{Y}$ & & Q 13. & Psychomotor agitation & -0.456 & 0.011 \\
\hline & & Q 15. & Irritability & -0.421 & 0.021 \\
\hline
\end{tabular}

The above table indicated that the Anxiety symptoms namely, Headache, neck pain, back pain, weakness, fast heart beat and hot blushing face ; and Depression symptoms such as, depressed affect, Tachycardia, fatigue, Psychomotor agitation, irritability factors were negatively correlated $(\mathrm{p}<0.05)$ with the social domain of WHOQOL-Bref questionnaire thereby affecting the personal relationship in the Radiotherapy group.

\section{Discussion}

In a study conducted by Zhen Guo et al. (2013), it was observed that during Radiotherapy treatment $(n=89)$ enrolled patients were affected by anxiety $(52 \%)$ and depression $(48 \%)$, as assessed by ZSAS and ZSDS, respectively ${ }^{[4]}$. Of these patients, women suffered from more anxiety (61\%) and depression (53\%) than men (anxiety, 39\%; depression, 38\%) ${ }^{[4]}$. The researcher used the European Organization for Research and Treatment of Cancer Quality of Life Questionnaire-Core 30 (EORTC QLQ-C30) to survey health-related QOL of cancer patients during Radiotherapy treatment ${ }^{[4]}$. In a study conducted by Luciano J. Pereira et al.(2015) on a group of 30 cancer patients, it was observed that while measuring QOL through WHOQOL-Bref, significant differences between cancer patients and controls for the Social Relationship and Environment domains and also for the overall QOL were

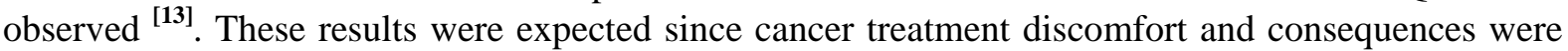
known to promote changes in physical and emotional integrity and loss of self-esteem, with consequent reduction in quality of life ${ }^{[13]}$. Negative impact was observed on the psychological and environmental domains of the assessed individuals ${ }^{[13]}$. In a study conducted by Novin Nikbakhsh,et al. (2014) on 150cancer population undergoing Chemotherapy or Radiotherapy, high frequency of significant association was observed in the patients who received chemotherapy as a single treatment (66.7\% had symptomatic depression and $77.8 \%$ symptomatic anxiety) as assessed by hospital anxiety and depression scale (HADS)developed by Zigmond and Snaith in 1983 that wasbased on a four point 14-item HADS with two subscales for anxiety (seven items) and for depression (seven items) ${ }^{[12]}$. Pandey et al's study on 117 patients with cancer revealed that $23 \%$ of patients who received Chemotherapy had depression ${ }^{[17]}$. Cazzaniga et al. Showed that radiotherapy in patients with cancer caused depression and anxiety ${ }^{[17]}$. Souza et al. Studied 102 patients with cancer under chemotherapy and observed that $10.8 \%$ and $1.9 \%$ of them had moderate and severe depression, respectively ${ }^{[17]}$. In a study conducted by Zahra Shayan et al. (2014) on 260 patients with cancer, 46(18.5\%) had borderline 
Texila International Journal of Clinical Research

Volume 3, Issue 2, Dec 2016

depression, 46(18.5\%) had slight depression, 52(20.9\%) had moderate depression 15(6\%) had severe depression, and 5(2\%) had very severe depression as assessed by the Beck questionnaire that consisted of 21 items ${ }^{[17]}$. In a study conducted by Preeti (2015) on 40 cancer patients out of which 20 underwent chemotherapy sessions and 20 patients underwent Radiotherapy, revealed that anxiety level was high in patients treated with Radiotherapy and depression level was high in patients treated with Chemotherapy ${ }^{[19]}$. The results of the present study investigations allowed the investigator to state that QLQ with counseling could play a key role in the life of cancer patients through the resulting improvement in their QOL. This fact was supported by the study conducted by Hogan et al. who observed that 73 studies reported some benefit of social support provided by friends and families to patients with chronic conditions due to cancer or cancer treatment related side-effects [18]. Unfortunately, cancer caregivers often lack the skills and resources they need to help the patient manage their treatment and the negative consequences of their disease and its management ${ }^{[18]}$.

According to the investigator's knowledge, these preliminary findings were among the first to illustrate the association of the anxiety and depression levels with quality of life of cancer patients during inpatient chemotherapy or outpatient radiotherapy treatment using the QOL assessment tools: WHOQOL-Bref, ZSAS, and ZSDS in the form of questionnaire.

\section{Conclusion}

In Indian scenario, population differs in ethnic, social, cultural as well as economical aspects as compared with western population, so it was necessary to study the factors of anxiety and depressionthat affected QOL especially of Indian cancer patients. Chemotherapy, and Radiotherapy treatment for cancer could dramatically effect body and mind of the cancer patient receiving treatment. In the present study, it was observed that Moderate to Severe levels of Anxiety significantly affected the activities of daily living and work capacity of the Physical health domain; and Security and Leisure activity of the Environmental domain in the chemotherapy group patients, and the personal relation factor of Social relationship domain in the radiotherapy group patients. On the other hand, Mild levels of Depression affected the Social support factor of Social relationship in the chemotherapy group and personal relation factor of Social relationship domain in the radiotherapy group. Therefore, further investigation about this topic could be a great help to treatment of anxiety and depression in patients with cancer undergoing either of the treatment. The results suggested that WHOQOL-Bref, ZSAS, and ZSDS could be used as a reliable predictor of association and correlation of anxiety and depression with that of quality of life in the cancer patients. Moreover, the WHOQOLBref, ZSAS, and ZSDS were observed to be psychometrically sound tool with moderate internal consistency that could be used in English language as well as, Hindi language in the cancer population of North India. Patients on chemotherapy were more badly affected in some domains of quality of life, compared to those on radiotherapy. In cancer, even with limited resources, an impact could be achieved if the right priorities and strategies are established and implemented. Thus, the social origin of lifestyle must be considered in management of QOL of cancer patients. Anxiety and Depression affected the Physical, Social and Environmental domains the most in chemotherapy group. On the other hand, Anxiety and Depression affected the Social domain the most in the radiotherapy group. Thus, it was observed that though levels of anxiety and Depression were inversely (negatively) correlated with the domains of Quality of life of cancer patients during chemotherapy or radiotherapy, yet the relationship was significant. This study showed that the increase in anxiety or depression level subsequently reduced or decreased the QOL of the cancer patients either undergoing Chemotherapy or Radiotherapy treatment. The present study showed that Anxiety and Depression could be used as Predictors or Determinants of QOL of cancer patients during the chemotherapy session or radiotherapy sessions. Thus, there is no "gold standard" of QOL measurement because no one instrument would be appropriate for all situations. So, the quality of life is influenced by how the patient view what is happening to them right at this very moment, i.e., during the treatment modality (Chemotherapy or Radiotherapy) sessions. 


\section{Future directions}

i. To investigate the usefulness and feasibility of QLQ intervention, further work, including a prospective longitudinal multicenter study, is recommended.

ii. Psycho-Oncology Clinic should be made functional at every hospital for the needy cancer patients once a week. Frequency should be increased depending upon the requirement.

iii. Recommendations for future studies include qualitative studies with larger sample size so that the data can be generalized across a broader population regarding Quality of life interventions for providers and how such interventions can improve patient outcomes.

iv. An optimum level and high quality of care for patients with different types of cancer treated for cancer could be achieved by frequent and regular measurement of the quality of life of cancer patients through QLQ.

v. There is need for media attention, further research in QOL, strong participation of nongovernmental organizations and care groups to cope the disease for long- term survivors.

vii. Finally, an interventional study focusing on the spiritual and social needs of the patient as well as pain and symptom management could be implemented in a hospital setting.

\section{Limitations of the study}

i. Study was delimited to small sample size of 60 patients receiving chemotherapy $(n=30)$ and radiotherapy $(\mathrm{n}=30)$ in the age group of 18-75 years, which might cause potential sampling errors.

ii. The study lacked active control groups. The results would have been different if the same individual who underwent chemotherapy also undergoes through radiotherapy treatment; therefore, it was difficult to find a matched group as control for comparison. Using the pre-treatment status as the internal control and following up its changes over time would be more appropriate method for comparison.

iii. The duration of time of the research study evaluation for anxiety and depression through QLQ was short, which meant that it was unable to determine how mood swings and behavioral changes in cancer patients would take place in the time after the end of Chemotherapy or Radiotherapy treatment.

iv. This study was a restricted one because it was a single-centered, cross-sectional study in which the cause and effect relationship was not established.

\section{Footnotes}

Author's Contribution: 1- acquisition of data; 2- analysis and interpretation of data; 3- drafting of the manuscript; 4- critical revision of the manuscript for important intellectual content; 5- statistical analysis; 6- administrative, technical and material supports.

Funding/Support: Self-funded.

\section{Acknowledgement}

The present study is a part of the research programme, “Assessment of Quality of Life of Adult Cancer Patients during inpatient Chemotherapy Treatment (CT), or outpatient Radiotherapy Treatment (RT) in a tertiary care hospital of New Delhi, India”. This research was supportedand assisted by at:

Department of Medical Oncology and Radiation Oncology, Dr. B.L. Kapur Memorial Hospital, New Delhi, India.

Under the Supervision of:

Dr. S. Hukku (H.O.D. of Radiation Oncology Department),

Dr. B.L. Kapur Memorial Hospital,

New Delhi, India.

Dr. Amit Agarwal (H.O.D. of Medical Oncology Department),

Dr. B.L. Kapur Memorial Hospital,

New Delhi, India. 
Texila International Journal of Clinical Research

Volume 3, Issue 2, Dec 2016

Under the Guidance of:

Dr. Renita Bhamrah (Texila American University),

E-mail:darpreetrenita@gmail.com

\section{References}

[1]. Bronson, T. (1999). "Quality of Life of the Hemodialysis Patient". Masters Theses. Paper 531. Retrieved from: http://scholarworks.gvsu.edu/theses/531

[2]. Damodar, G., Gopinath, S., Vijayakumar, S., \& Rao, A. Y. (2014). Reasons for Low Quality of Life in South Indian Cancer Patient Population: A Prospective Observational Study. Indian Journal of Pharmaceutical Sciences, 76(1), 2-9.

[3]. Deshpande, P. R., Sheriff, M. K., Nazir, A., Bommareddy, S., Tumkur, A., Naik, A. N. (2013). Patientreported quality of life outcomes in Indian breast cancer patients: Importance, review of the researches, determinants and future directions. J Can Res Ther, 9: 11-6. Doi: 10.4103/0973-1482.110341

[4]. Guo, Z., Tang, H. Y., et al. (2013). The benefits of psychosocial interventions for cancer patients undergoing radiotherapy. Health and Quality of Life Outcomes, 11:21. doi: http://10.1186/1477-7525-11-121 [5]. Koller, M., Lorenz,W., Wagner, K., Kell, A., Trott, D., Engenhart-Cabillic, R., Niles, C. (2000). Expectations and quality of life of cancer patients undergoing radiotherapy. Journal of the Royal Society of Medicine, 93:621-628.

[6]. Manoharan,N., Tyagi, B. B., Raina, V. (2009). Cancer Incidences in Urban Delhi- 2001-05. Asian Pacific Journal of Cancer Prevention, 10, 799-806.

[7]. Mansano-Schlosser, Cristina, T. \& Ceolim, Filomena, M. (2012). Quality of life of cancer patients during the chemotherapy period. Texto \& Contexto - Enfermagem, 21(3), 600- 607. https://dx.doi.org/10.1590/S010407072012000300015

[8]. Marimuthu, P. (2008). Projection of cancer incidence in five cities and cancer mortality in India. Indian J Cancer, 45, 4-7. Doi: 10.4103/0019-509X.40639.

[9]. Nagy, J. K. (2011). Quality of life of head and neck cancer patients after tumor treatment and subsequent maxillofacial rehabilitation. (Thesis). University of Szeged, Hungary. Retrieved from: http://doktori.bibl.uszeged.hu/1349/1/K\%C3\%A1d\%C3\%A1r-Nagy_DDS_-_Ph_D_Thesis.pdf

[10]. Nair, M. K., Varghese, C., Swaminathan, A. (2005). Cancer: Current scenario intervention strategies and projections for 2015. In:Burden of Disease in India, Background Papers. New Delhi: National Commission for Macroeconomics and Health, Ministry of Health and Family Welfare, Government of India; 218-225.

[11]. Ng, W. (2004). Quality of life of nasopharyngeal carcinoma patients treated with nasopharyngectomy. (Thesis). University of Hong Kong, Pokfulam, Hong Kong SAR. Retrieved from http://dx.doi.org/10.5353/th_b3197208

[12]. Nikbakhsh, N., Moudi, S., Abbasian, S., \& Khafri, S. (2014). Prevalence of depression and anxiety among cancer patients. Caspian Journal of Internal Medicine, 5(3), 167-170.

[13]. Pereira, L. J., Braga Caputo, J., Midori Castelo, P., Francelino Andrade, E., Silva Marques, L., Martins de Paiva, S., Márcia Pereira, S., Vicente Pereira, C. (2015). Oral physiology and quality of life in cancer patients. Nutr Hosp, 31(5):2161-6. Doi: 10.3305/nh.2015.31.5.8565.

[14]. Raoof, A., Yacoub, S., Asaad, Y., \& Al- Hadithi, T. (2015). Quality of Life Among Cancer Patients Treated With Chemotherapy or Radiotherapy in Erbil City An Evaluation Study. Cancer And Clinical Oncology, 4(1), 19-27. doi: http://dx.doi.org/10.5539/cco.v4n1p19

[15]. Rustoen, T. (1995). Hope and Quality of life, two central issues for cancer patients: a theoretical analysis. Cancer Nurs, 18(5):355-61.

[16]. Saha, D. (2016, June 1). Delhi has the highest cancer incidence in India. Retrieved October 13, 2016, from newslaundry.com: http://www.newslaundry.com/2016/06/01/ delhi-has-the-highest-cancer-incidence-in-india/\# [17]. Shayan, Z., Shahkolahi, Z., Ahmadlo, N., Vafaee, L., Shayan, L. (2014). Prognostic Factors of Depression in Patients With Cancer Undergoing Chemotherapy and Radiotherapy. Health Scope, 3(4).e18609. Doi: 10.17795/jhealthscope-18609 
[18]. Silveira, M. J., Given, C. W., Cease, K. B., Sikorskii, A., Given, B., Northouse, L. L., \& Piette, J. D. (2011). Cancer Carepartners: Improving patients’ symptom management by engaging informal caregivers. $B M C$ Palliative Care, 10, 21. http://doi.org/10.1186/1472-684X-10-21

[19]. Srivastav, P. (2015). Psychological Distress Among Head and Neck Cancer Patients Treated With Chemotherapy and Those Treated With Radiotherapy. Research and Reviews: Journal of Oncology and Hematology (RRJoOH), 4(1), 23-28.

[20]. Sunderam, S., Haider, S., Kumar, M., Kashyap, V., \& S., J. (2016) Assessment of quality of life of cancer patients attending oncology clinic in a tertiary care hospital of Jharkhand, India. International Journal of Community Medicine and Public Health, 3(1), 281-286. doi:10.18203/2394-6040.ijcmph20151577.

[21]. Torre, L. A., Bray, F., Siegel, R. L., Ferlay, J., Lortet-Tieulent, J. and Jemal, A. (2015). Global cancer statistics. CA: A Cancer Journal for Clinicians, 65: 87-108. doi:10.3322/caac.21262

[22]. Ustundag, S., Zencirci, A. D. (2015). Factors affecting the quality of life of cancer patients undergoing chemotherapy: A questionnaire study. Asia Pac J Oncol Nurs, 2:17-25. Doi: 10.4103/2347-5625.152402 [23]. WHOQOL Group. (1998). Development of the World Health Organization WHOQOLBREF quality of life assessment. Psychol Med, 28:551-8.

[24]. Zung, W. W. K. (1971). A rating instrument for anxiety disorders. Psychosomatics, 12(6), 371-379. Available at: https://psychology-tools.com/zung-anxiety-scale/

[25]. Zung, W. W. K. (1965). A Self-Rating Depression Scale. (1965). Arch Gen Psychiatry, 12: 63-70. Available at: https://psychology-tools.com/zung-depression-scale/ 


\title{
Diabetes Education Program Increases Knowledge, Reduces Obesity and better Glycemic Control in Central Hospital Nampula
}

\author{
Article by Madhumati Varma \\ MMSC-Diabetology, Texila American University \\ Email: madhumativarma@gmail.com
}

\begin{abstract}
Background: The patient of diabetes in Central hospital Nampula has the prevalence of obesity, poor knowledge regarding diabetes, completely depends on the diabetic pharmacological treatment and lack of awareness of complication of diabetes. There is a need of lifestyle modification, increase knowledge of diabetes to improve obesity, glycaemic control, reduce its complication.

Methods: This is clinical intervention study, 648 of the participants of diabetes mellitus taken for study in out-patient diabetic clinic in hospital central Nampula, according to inclusion and exclusion criteria, participated in the pre-test at baseline and post-test after the second follow up session of education, during each session of education body mass index and fasting blood sugar were recorded. Education commenced with instruction in groups of each session followed by individual advice sessions for each patient with different specialists.

Results: The present study found that educational intervention of diabetes was highly effective to increase knowledge of diabetes with compare of pre-test and Post-test score $(P<.001)$, fasting blood sugar and body mass index significantly decreased from baseline in the second follow up $(P<.001)$. Age was significantly correlated with body mass index and fasting blood sugar $(P<.001$, ) Posttest with body mass index and fasting blood sugar were significantly correlated $(P<.01)$. A post hoc Turkey test on body mass index when compared with fasting blood sugar found significantly $(P=.05)$ at baseline, at first follow up $(P=.005)$ and at second follow up $(P=.005)$.

Conclusion: The present study found that educational intervention was highly effective in controlling body mass index, fasting blood sugar and improves knowledge of diabetes among participants of diabetes mellitus.
\end{abstract}

Keywords: Diabetes Mellitus, blood sugar, body mass index, effect education, control, participants

\section{Introduction}

\section{Background}

\section{World wide}

The Global report from the World Health Organization (WHO), published in 2016, estimated that 422 million of the adult population lives with diabetes the number of diabetic patients has dramatically increased 4 times over in the adult population, compared to 108 million in 1980. Diabetes Mellitus is a chronic metabolic disorder, which is caused by partial deficiency or total deficiency of insulin. Diabetic mellitus type- 1 has complete deficiency of insulin and diabetes type-2 has partial deficiency of insulin with receptor of insulin not functioning properly to facilitate enter glucose into cells for utilization and formation of units of energy. WHO has estimated that the number of diabetic patients will double by 2030.Diabetes is increasing more rapidly in low and medium income groups than higher income groups as well as in developing countries compared to Europeanian countries, has less prevalence of diabetes. The top five countries with the highest prevalence of diabetes. Include the following: India, China, USA, UK, Brazil and Indonesia. Diabetes Type 1 most common in Scandinavian populations, Sardines, and Kuwait, and less common in Asia, Latin and European population. 
Texila International Journal of Clinical Research

Volume 3, Issue 2, Dec 2016

\section{Mozambique}

Mozambique is located on the East coast of Africa (Wikipedia 2016). There are 274,700 diabetic patients and 9716 deaths due to diabetes, according to a report of 2015 (IDF 2015). This country is the setting for this study. There is the prevalence of obesity, poor knowledge regarding diabetes and lack of awareness of complication of diabetes. Most of the population uses traditional healers for treatment of diabetes. There are unhealthy dietary habits, sedentary lifestyle in urban population and increased economic growth amongst professions related to office work, which is one of the risk factors that causes diabetes and its complications. Among the group of patients that seek care in public hospitals, many are poor and cannot afford the cost of medication or healthy foods. There is an 80 dollar expenditure allotted to each patient of diabetes from the country's Ministry of Health. Additionally, the ministry of health provides free medication for all chronic diseases, including diabetes and hypertension. Currently, there is no study that has been done on the effects of education in various modalities of diabetes in clinical practice. Accordingly, there is an extreme need to educate patients of diabetes to improve diabetic control and reduce its complication.

\section{Objective and hypothesis, problem, purpose, question statement}

\section{Problem}

The population of this study are diabetic patients in the Central Hospital Nampula in Mozambique, who are from low and medium income groups. This group of patients has limited sources of incomes and, completely depends on the diabetic pharmacological treatment of the government hospital pharmacy, which gives medication free of cost. In the country of Africa, there is a generally poor health education regarding the facility of diabetes. There are no professional diabetic health educators and patients receive advice from doctors and dieticians regarding their diets and directions on how to take their medicine to continue treatment at home. Due to the large size of patient loads in outpatient consultations with diabetes, it is not possible to sit with each patient and provide specific health education about diabetes. Also, these groups of patients do not access of the internet to improve their own self-education from different sources. It is clear that when diabetic patients only utilize pharmacological treatments that it is not sufficient to control diabetes and complication (Association, 2002).

\section{Objective}

1. There is a need for lifestyle modification, increase knowledge of diabetes and its complication, to improve ability to patient detects small complication and present physician, adherence of treatment.

2. There are different categories of patients, and levels of controlled diabetic patients, there for the different types diabetes requires different types of education, which can be depending on associated complications and diseases.

There following are the clinical categories patients:

1. Good controlled over blood sugar and without complication.

2. Fair/not controlled blood sugar with or without complication.

3. Good control of blood sugar and without complication, but other disease example HIV treatment, CVA etc.

\section{Question statement}

To conduct the study, used dependent variable fasting blood sugar and independent age, body mass index, pre-test and post-test scores.

Positive hypothesis, the positive correlation between controls of fasting blood sugars change of lifestyle modification includes diet and exercise. The positive correlation between controls of fasting 
blood sugars to improve body mass index. The knowledge of diabetes could help in controlling diabetes and blood sugar in case implemented knowledge of diabetes in life style.

Null hypothesis, The various reasons which can responsible for uncontrolled fasting blood sugar, negatively correlated to nonadherence of treatment, economic crisis to buy medicine, laboratory unable to provide results of fasting blood sugar, traditional healer involved in treating diabetes as a choice of patients.

\section{Method}

This study was conducted on regular patients of the diabetic of outpatient department the Central Hospital of Nampula. The study investigated the effects of three sessions of the diabetes education program (baseline, first follow-up and second follow-up) on each patient at one-month intervals. The inclusion and exclusion criteria for participation in the education program are listed below.

\section{Inclusion and exclusion criteria for the study}

Table 1. Inclusion and exclusion criteria for the study

\begin{tabular}{|l|l|}
\hline Inclusion criteria & Exclusion criteria \\
\hline $\begin{array}{l}\text { Diabetic patients of consultations of Central } \\
\text { hospital Nampula }\end{array}$ & Participates completed 3 sessions \\
\hline Mozambican citizen & Not willing to participate \\
\hline Any age & $\begin{array}{l}\text { Usability of participants to attend session } \\
\text { each month }\end{array}$ \\
\hline
\end{tabular}


Texila International Journal of Clinical Research

Volume 3, Issue 2, Dec 2016

\section{Conceptual framework}

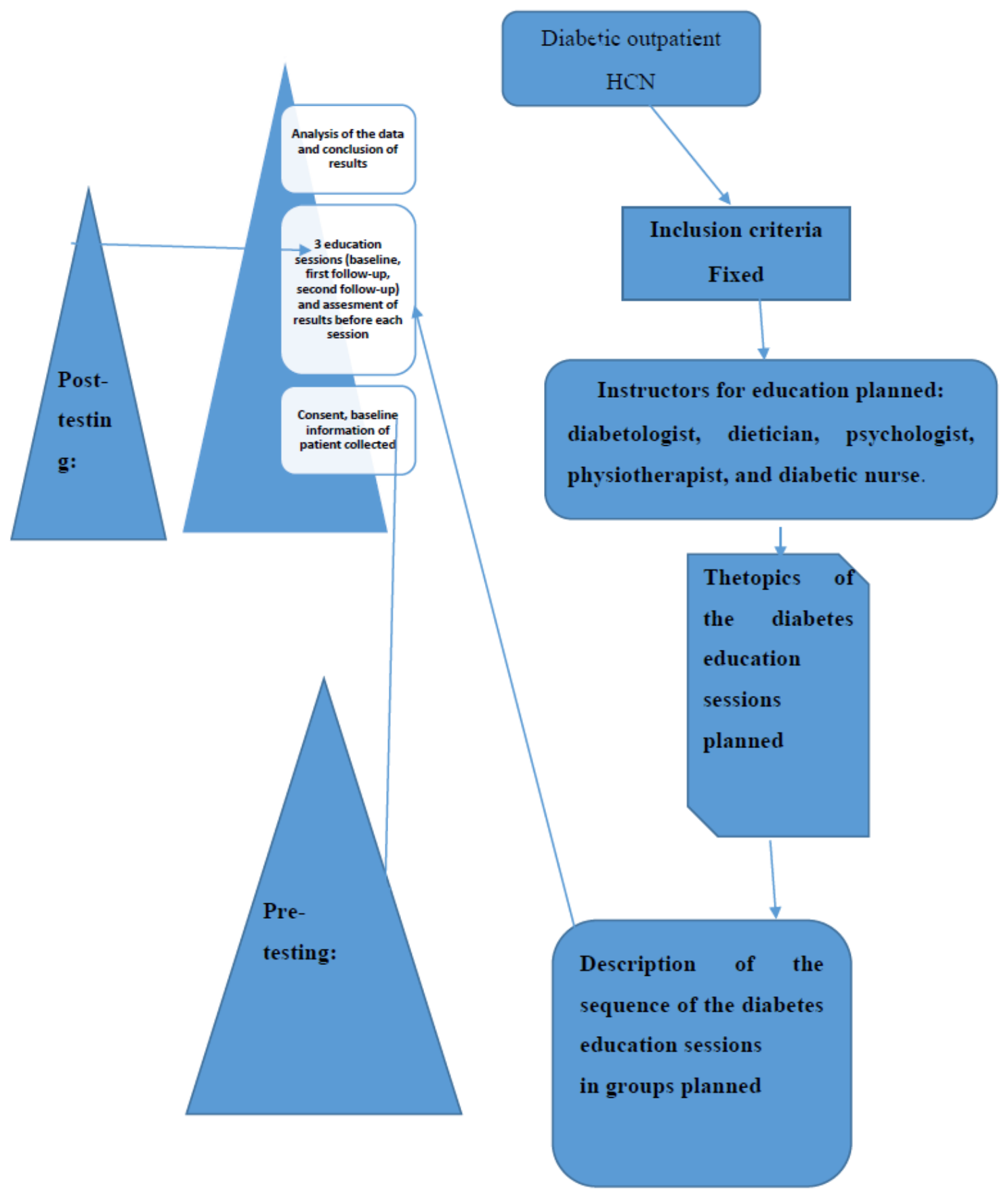

Figure 1. Conceptual framework

\section{Sample number and characteristics}

A sample of 648 participants was taken for this study. The inclusion criteria for participating in the diabetes education program dictated that patients should be of the OPD of diabetes, willing to participate in the education sessions and willing to give consent to be included in the study. 
Participants were excluded if they had already completed three sessions of education or if they lived in a district that made it impossible for them to return to one month for the next education session.

\section{Organization of education sessions}

Instructors: Amongst the group instructors were a diabetologist, dietician, psychologist, physiotherapist, and diabetic nurse.

\section{Tasks had to be performed before each of the sessions}

\section{The baseline session}

The diabetic nurse took consent and accessed the patient's clinical history before conducting a physical examination that included checking vital signs and body mass index. The physical examination was to be done by a physician. They also noted the results of a blood analysis that tested for sugar, which had been conducted 2-3 days prior to the education session. The participants answered questions based on knowledge of diabetes in pre-test questioner before start of baseline session of education of diabetes to see how much knowledge they have of diabetes.

\section{First follow up}

At the beginning of the session, the diabetic nurse measured the body mass index and noted the results of the blood analysis that tested for sugar, which had been conducted 2-3 days prior to the education session.

\section{Second follow up}

At the beginning of the session, the diabetic nurse measured the body mass index and noted the results of the blood analysis that tested for sugar, which had been conducted 2-3 days prior to the education session. The participants answered same questions based on knowledge of diabetes in posttest questioner after complete second follow up session of education of diabetes to see how much knowledge they increased after completion of diabetes education.

\section{Methodology of education}

The education sessions were in the form of a conference on all specialties leading a class of various forms verbal, demonstrating real situations, operating the apparatus and discussing difficulties, if any at the end of the session.

\section{Pre- and post-testing}

There was a pre-test questionnaire that aimed to assess the existing knowledge of diabetes before starting the baseline education session. The same questions were asked after the completion of the second follow-up education session.

The format of the pre- and post-tests given before and after the education sessions:

Name of patient 
Texila International Journal of Clinical Research

Volume 3, Issue 2, Dec 2016

Answer the questions on

\begin{tabular}{|l|l|l|}
\hline Questions & $\begin{array}{l}\text { Answer before } \\
\text { education } \\
\text { session }\end{array}$ & $\begin{array}{l}\text { Answer after } \\
\text { education } \\
\text { session }\end{array}$ \\
\hline $\begin{array}{l}\text { 1 Diabetes mellitus is characterized by } \\
\text { hyperglycemia/increased sugar levels, recurrent or } \\
\text { persistent in blood. }\end{array}$ & Yes / No & Yes / No \\
\hline 2. Type 1 Diabetes is more frequent at 5 to 15 years of age. & Yes / No & Yes / No \\
\hline $\begin{array}{l}\text { 3. Symptoms of diabetes include the frequent urge to } \\
\text { urination, hunger, thirst, weight loss, and weakness. }\end{array}$ & Yes / No & Yes / No \\
\hline $\begin{array}{l}\text { 4. Glibenclamida is an oral antidiabetic medication side } \\
\text { effects feeling hungry. }\end{array}$ & Yes / No & Yes / No \\
\hline $\begin{array}{l}\text { 5. A healthy diet of diabetics is poor in greens vegetables, } \\
\text { but is rich in sugar and fried foods. It is also healthy to eat } \\
\text { food once a day. }\end{array}$ & Yes / No & Yes / No \\
\hline $\begin{array}{l}\text { 6. The benefits of exercise are a decrease in body weight } \\
\text { and the use of sugar in cells without insulin. }\end{array}$ & Yes / No & Yes / No \\
\hline $\begin{array}{l}\text { 7. Anxiety and depression increase blood sugar } \\
\text { level/uncontrolled diabetes. }\end{array}$ & Yes / No & Yes / No \\
\hline 9. Insulin should not be frozen. & Yes / No & Yes / No \\
\hline $\begin{array}{l}\text { 10. Symptoms of hypoglycaemia/decreased blood sugar are } \\
\text {-tremors, palpitations, hungry, and sweating. }\end{array}$ & Yes / No & Yes / No \\
\hline
\end{tabular}

Pre-test mark

Post-test mark-

Figure no 2:The format of the pre- and post-tests

\section{Description of intervention in health education}

\section{Description of the specialists that participated in giving the education sessions}

The different types of specialists involved in the health education intervention were:

1. The psychologist oriented the patients with basic aspects of psychology to help them live with diabetes.

2. The diabetologist provided knowledge about the general concepts of diabetes.

3. The dietician provided information about the specific diet needed for diabetic patients.

4. The physiotherapist demonstrated important exercises that were specifically for sufferers of diabetes and were applicable to all individuals.

\section{Group and individual education sessions}

After a three-hour group education session, individual outpatient consultations were conducted in different rooms with different specialists. These patients could consult a diabetologist, dietician, physiotherapist, or psychologist individually according to the specific needs of each patient.

\section{Organization of health education sessions}

Due to the shortage of separate spaces large enough to accommodate the number of participants in the baseline, first follow-up and second follow-up, sessions were limited to approximately 40-50 participants. To make education sessions more interesting and effective the beginning of each session was realized with baseline participants followed by first follow-up participants, and then second follow-up participants were taken for education of diabetes. There was no restriction on participants if they wanted to attend the same session twice. 
Each session built upon the knowledge gained in the previous session. The health outcomes of each patient were assessed in outpatient service to monitor the implementation of the knowledge imparted in the education sessions in daily life.

\section{Description of educational materials and information provided}

The topics of the sessions were chosen according to local culture, socioeconomic conditions, beliefs, lifestyle, common complications and associated diseases. The aim was to improve the outcomes of diabetes patients and prevent complications. The information was presented in a manner that the participants could easily understand and would motivate them to implement the new information their daily lives.

\section{The topics of the diabetes education sessions}

- Definition of diabetes and types of diabetes

- Symptoms of hypo- and hyperglycemia, both complications of diabetes

- Management of diabetes by diet and physical activity

- Psychological assistance

- Brief knowledge of medicines that are available in the pharmacy of the hospital

- Management of hypoglycemic medication

- Complications of diabetes and early detection

- Dental and foot care

- Diabetic control in special situations such as during Ramadan or when travelling

- Blood sugar monitoring using a glucometer

- How and where to inject insulin and how to prevent complications

- How to prevent primary and secondary stages of diabetes

- Participation of family members if willing and/or required

\section{Description of the sequence of the diabetes education sessions}

\section{In groups}

1. Welcome address to participants from a diabetologist

2. First part of the education session: lessons on diabetes by, taught by a diabetologist

3. Second part of the education session: information on diet and nutrition, taught by a dietician

4. Third part of the education session: information on how to cope psychologically with the disease, taught by a psychologist

5. Fourth part of the education session: the benefits of exercise and demonstrations of general exercises that are applicable to all patients, taught by a physiotherapist

6. Fifth part of the education session: how to inject insulin and use a glucometer, taught by a nurse

On an individual basis:

7. Sixth part of education session: individual consultations with each patient with a diabetologist, dietician, psychotherapist, or physiotherapist to help participants with particular recommendations based on associated diseases and complications, if needed

\section{Patient interviews}

The patients were interviewed in order to gather the following information:

- Identification details

- Medical history/past clinical history

- Body mass index

- Drug history (type of oral hypoglycemic agents, injectable form antidiabetic agents, drugs to maintain blood pressure and lipids)

- Analysis of fasting blood sugar before each session 
Texila International Journal of Clinical Research

Volume 3, Issue 2, Dec 2016

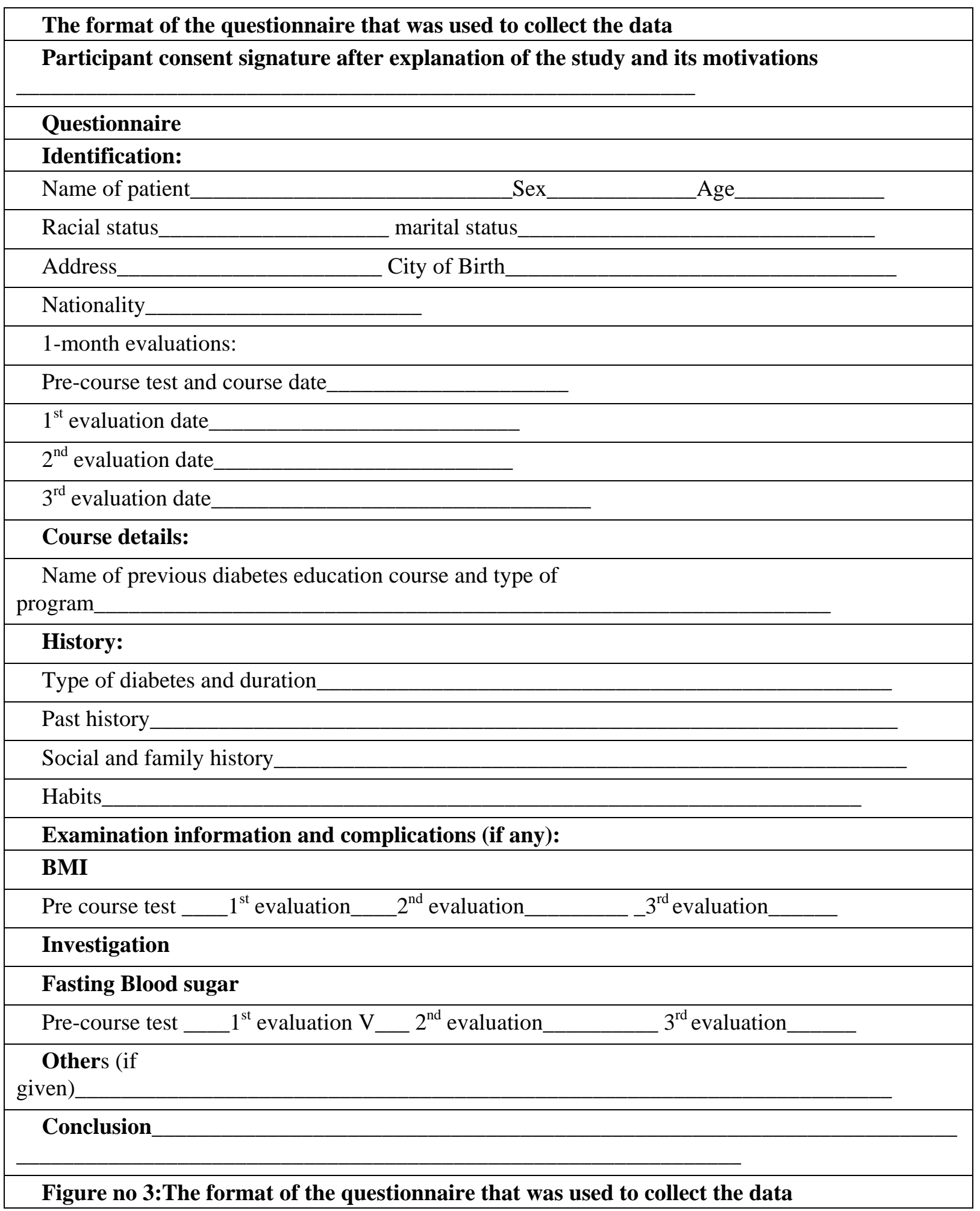

Variables used and their ranges

Age

21-40 years

41-60 years

61-80 years 
81-100 years

Information not given

\section{Pre-education test score}

Fair: $<5$

Good: 5-6

Very good: 7-8

Excellent: 9-10

Information not given

\section{Post-education test score}

Fair: $<5$

Good: 5-6

Very good: 6-8

Excellent: 9-10

Information not given

Body mass index (repeated for the baseline, first follow-up, and second follow-up)

Underweight: $0<18.5 \mathrm{Kg} / \mathrm{m} 2$

Normal: $18.5-24.9 \mathrm{Kg} / \mathrm{m} 2$

Overweight: $>25 \mathrm{~kg} / \mathrm{m} 2$

Pre obese: $25-29.9 \mathrm{~kg} / \mathrm{m} 2$

Obese: > $30 \mathrm{~kg} / \mathrm{m} 2$

Obese class 1: $30-34.9 \mathrm{~kg} / \mathrm{m} 2$

Obese class 2: $35-39.9 \mathrm{~kg} / \mathrm{m} 2$

Obese: $>40 \mathrm{~kg} / \mathrm{m} 2$

Information not given

Fasting blood sugar (repeated for the baseline, first follow-up, and second follow-up)

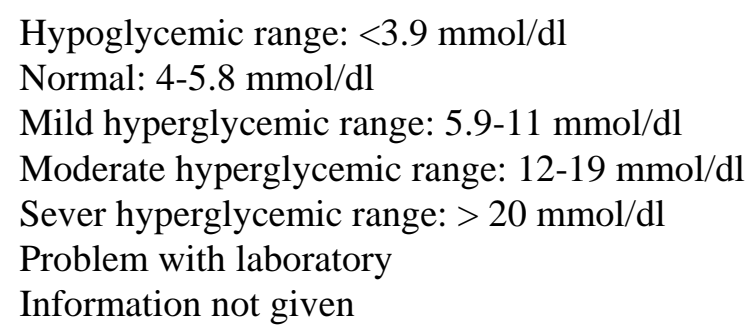

\section{Data analysis}

Statistical analysis was conducted with the Statistical Program for the Social Sciences (SPSS) version 22.0 software. Excel software was used for the formation of graphs using the SPSS results. The results were analyzed using descriptive analysis, such as percentages, means, and standard deviations of variables. The chi-square, Pearson correlations, degree of freedom, T-test, ANOVA, and post-hoc Turkey test were conducted to determine the relationship between the variables. A t-test, ANOVA, and p-value of less than 0.05 were considered to be significant. The tables and graphs below displaying the results.

\section{Results}

A sample of 648 participants was taken for this study. This study was conducted on regular patients of the diabetic outpatient department of the Central Hospital of Nampula. The study investigated the effects of three sessions of the diabetes education program (baseline, first follow-up and second follow-up) on each patient at one-month intervals. The inclusion criteria for participating in the 
Texila International Journal of Clinical Research

Volume 3, Issue 2, Dec 2016

diabetes education program dictated that patients should be in the OPD, willing to participate in the education sessions and willing to give consent to be included in the study. Participants were excluded if they had already completed three sessions of education or if they lived in a district that made it impossible for them to return to one month to the next education session. Amongst the group instructors were a diabetologist, dietician, psychologist, physiotherapist, and diabetic nurse. There was a pre-test questionnaire that aimed to assess the existing knowledge of diabetes before starting the baseline education session. The same questions were asked after the completion of the second follow up education session. There were various variables to assess from baseline to second follow up education session. The variables were assessed body mass index, fasting blood sugar.

\section{Descriptive analysis of Pre-test and post-test score at before baseline and after second follow up}

A sample of 648 patients with diabetes mellitus, those who had participated in educational sessions concerning diabetes mellitus, was taken for study in order to determine the effect of education on improving knowledge levels in diabetes. The analysis of knowledge increased to diabetes mellitus among participants was performed pre-test at the beginning baseline, and post-test at the end second follow up. The results are shown in Table 12, below. Briefly, the percentage of patients with fair level of knowledge (65.3\%) increased from beginning of baseline to very good level of knowledge of diabetes of $52.5 \%$ at the end of second follow up.

Table 2. Descriptive analysis of Pre-test and post-test score

\begin{tabular}{|l|l|l|l|l|l|}
\hline \multicolumn{2}{|c|}{} & Pre education Test Post education Test \\
\hline \multirow{3}{*}{ Valid } & Fair $<5$ & Frequency & Percentages & Frequency & Percentages \\
\cline { 2 - 6 } & Good 5-6 & 423 & 65.3 & 39 & 6.0 \\
\cline { 2 - 7 } & $\begin{array}{l}\text { Very Good 6- } \\
8\end{array}$ & 50 & 6.5 & 87 & 13.4 \\
\cline { 2 - 7 } & Excellent 9-10 & 2 & 7.7 & 340 & 52.5 \\
\cline { 2 - 7 } & Not done & 130 & .3 & 51 & 7.9 \\
\cline { 2 - 7 } & Total & 647 & 20.1 & 130 & 20.1 \\
\hline Missing & System & 1 & .2 & 647 & 99.8 \\
\hline Total & 648 & 100.0 & 648 & .2 \\
\hline
\end{tabular}

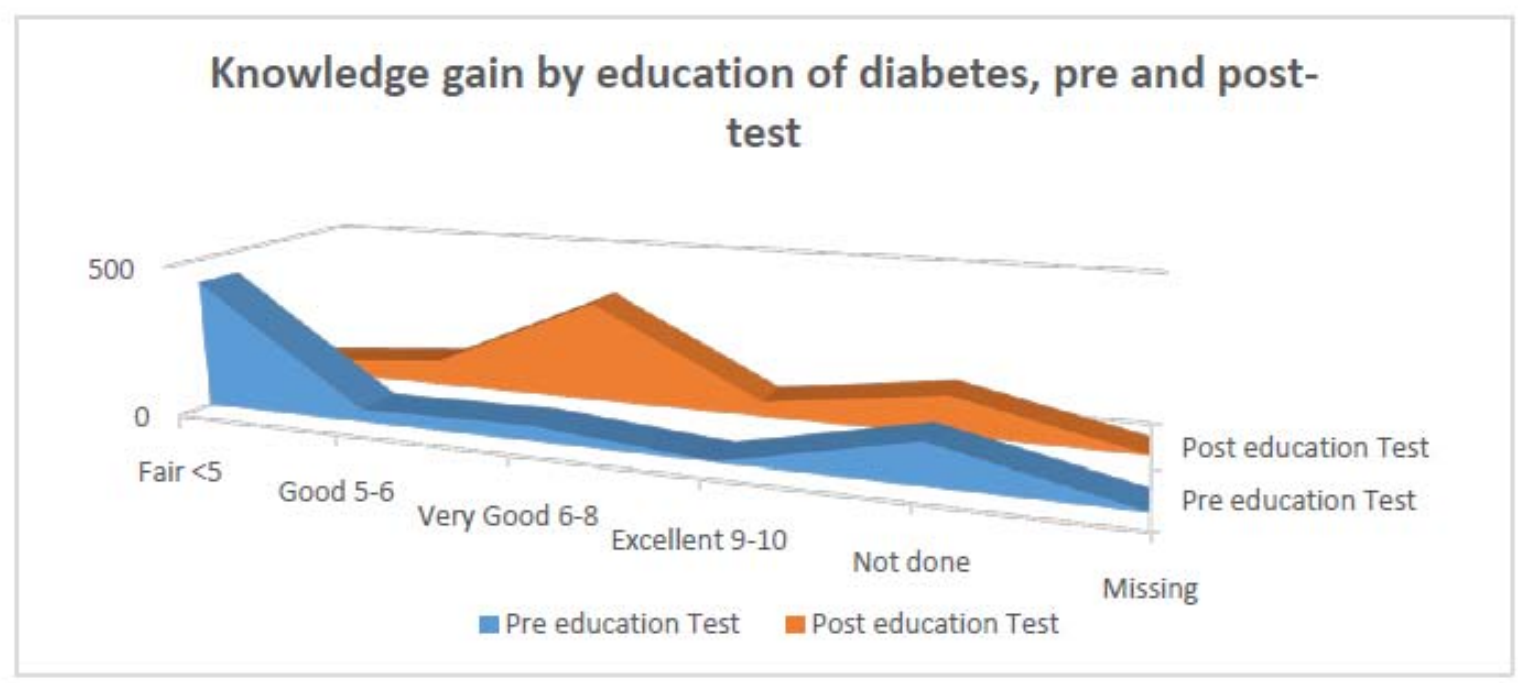

Figures 3: Linear chart: In the above charts we see that distribution of the compared pre-test and post-test scores at baseline and after second follow up after diabetes education among participants. 


\section{Descriptive analysis of body mass index of baseline, first follow up, second follow up}

A sample of 648 patients with diabetic mellitus, those who had participated in educational sessions concerning diabetes mellitus, was taken for study in order to determine the effect of education on improving body mass index levels in diabetes. The measurement of body mass index was performed at baseline, at the first follows up and at the second following up. The results are shown in Table 14, below. Briefly, percentage of patients with Underweight $<18.5 \mathrm{~kg} / \mathrm{m} 2$ decreased from baseline (4.5 $\%)$ to the first follows up (3.4\%), and to the second following up (2.5\%). The percentage of patients with pre obese $25-29.9 \mathrm{~kg} / \mathrm{m} 2$ were observed to decrease from baseline (35.2\%) to the first follows up (27.9\%), to the second following up 920.4\%). The percentage of patients with obese class 1 -30-34.9 $\mathrm{kg} / \mathrm{m} 2$ decreased from each following up from baseline (15.6\%) to the first follows up (13.1\%), to second following up (8.2\%). The percentage of patients with obese class 2- 30-34.9 kg/m2 decreased from each following up from baseline (5.7\%) to the first follows up (4.5\%), to the second following up $(2.8 \%)$ respectively.

Table 3. Descriptive analysis of body mass index at baseline, first follows up and second following up

\begin{tabular}{|c|c|c|c|c|c|c|}
\hline & Baseline l & Firs & fallow ul & Secc & id follow & \\
\hline Ranges of BMI & $\begin{array}{l}\text { Frequenc } \\
\mathrm{y}\end{array}$ & $\begin{array}{l}\text { Percent } \\
\text { ages \% }\end{array}$ & $\begin{array}{l}\text { Freque } \\
\text { ncy }\end{array}$ & $\begin{array}{l}\text { Percentage } \\
\mathrm{s} \%\end{array}$ & $\begin{array}{l}\text { Freque } \\
\text { ncy }\end{array}$ & $\begin{array}{l}\text { Percenta } \\
\text { ges \% }\end{array}$ \\
\hline $\begin{array}{l}\text { Underweight }<18.5 \\
\mathrm{~kg} / \mathrm{m} 2\end{array}$ & 29 & 4.5 & 22 & 3.4 & 16 & 2.5 \\
\hline Normal $18.5-24.9$ kg/m2 & 191 & 29.5 & 169 & 26.1 & 135 & 20.8 \\
\hline Overweight $>25$ kg/m2 & 9 & 1.4 & 9 & 1.4 & 11 & 1.7 \\
\hline Preobese $25-29.9$ kg/m2 & 228 & 35.2 & 181 & 27.9 & 132 & 20.4 \\
\hline Obese $>30 \mathrm{~kg} / \mathrm{m} 2$ & 6 & .9 & 7 & 1.1 & 10 & 1.5 \\
\hline $\begin{array}{l}\text { Obese class } 1 \text { 30-34.9 } \\
\mathrm{kg} / \mathrm{m} 2\end{array}$ & 101 & 15.6 & 85 & 13.1 & 53 & 8.2 \\
\hline $\begin{array}{l}\text { Obese class } 2 \text { 35-39.9 } \\
\text { kg/m2 }\end{array}$ & 37 & 5.7 & 29 & 4.5 & 18 & 2.8 \\
\hline Obese class3 $>40 \mathrm{~kg} / \mathrm{m} 2$ & 13 & 2.0 & 6 & .9 & 4 & .6 \\
\hline Not done & 32 & 4.9 & 138 & 21.3 & 267 & 41.2 \\
\hline Total & 646 & 99.7 & 646 & 99.7 & 646 & 99.7 \\
\hline Missing & 2 & .3 & 2 & .3 & 2 & .3 \\
\hline Total & 648 & 100.0 & 648 & 100.0 & 648 & 100.0 \\
\hline
\end{tabular}




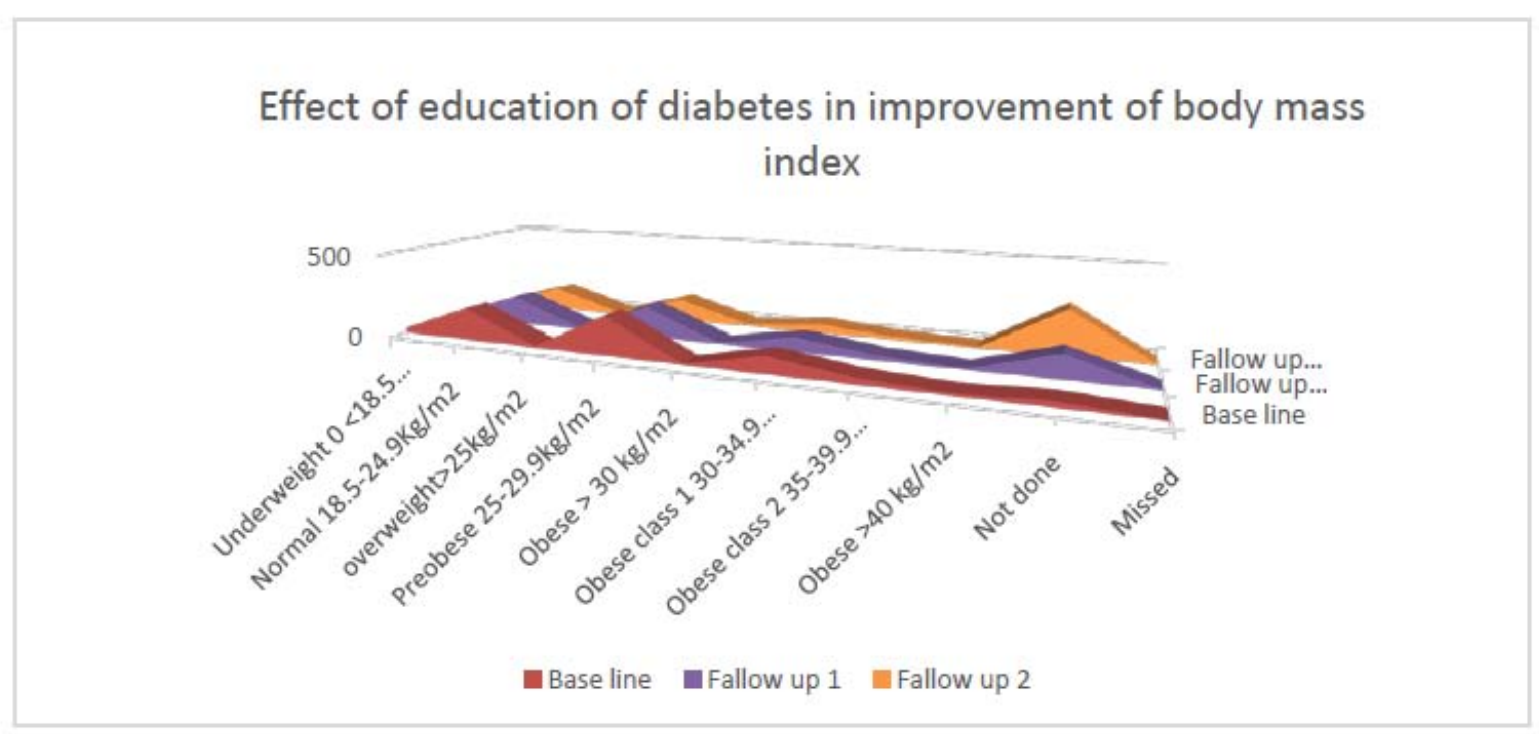

Figures 4. Linear chart: In the above charts we see that distribution of the compared body mass index at baseline, first follows up, and second following up after education among participants.

\section{Descriptive analysis of fasting blood sugars at baseline, first follows up and second following up}

A sample of 648 patients with diabetes mellitus, those who had participated in educational sessions regarding diabetes mellitus, was taken for study in order to observe effect of education on improving fasting blood sugar concentration of diabetes. The analysis of fasting blood sugar levels was performed at baseline, at the first follows up and at the second following up. The results are described in Table 15, below. Briefly, the percentage of patients with hypoglycemic concentrations $<3.9$ $\mathrm{mmol} / \mathrm{dl}$ improved on baseline (4.8\%), to the first follows up (4.2\%), to the second following up (5.1\%). The percentage of patients with a normal fasting blood sugar range of 4.0-5.8 mmol/dl decreased from baseline (21.8\%), to the first follows up (19.0\%), to the second following up (15.0\%). The percentage of patients with mild hyperglycemic concentrations of 5.9-11.0 mmol/dl decreased from each following up from baseline (41.4\%), to the first follows up (37.0\%), to the second following up (28.2\%). The percentage of patients with moderate hyperglycemic levels of 12.0-19.0 $\mathrm{mmol} / \mathrm{dl}$ were observed to decreased at each following up from baseline (22.2\%), to first follows up (17.9\%), to second following up (12.5\%). The percentage of patients with severe hyperglycemic levels of $>20.0 \mathrm{mmol} / \mathrm{dl}$ decreased from baseline (7.9\%), to the first follows up (4.5\%), to the second following up (3.2\%). 
Table 4. Descriptive analysis of fasting blood sugar at baseline, first follows up and second following up

\begin{tabular}{|l|l|l|l|l|l|l|}
\hline & \multicolumn{2}{|l|}{ Baseline line } & \multicolumn{2}{l}{ First follow up } & \multicolumn{2}{l|}{ Second follow up } \\
\hline $\begin{array}{l}\text { Ranges of Blood } \\
\text { Sugar }\end{array}$ & $\begin{array}{l}\text { Frequen } \\
\text { cy }\end{array}$ & $\begin{array}{l}\text { Percentag } \\
\text { es \% }\end{array}$ & $\begin{array}{l}\text { Frequenc } \\
\text { y }\end{array}$ & $\begin{array}{l}\text { Percentag } \\
\text { es \% }\end{array}$ & Frequency & $\begin{array}{l}\text { Percentag } \\
\text { es \% }\end{array}$ \\
\hline $\begin{array}{l}\text { Hypoglycaemic }<3.9 \\
\text { mmol/dl }\end{array}$ & 31 & 4.8 & 27 & 4.2 & 33 & 5.1 \\
\hline Normal 4-5.8 mmol/dl & 141 & 21.8 & 123 & 19.0 & 97 & 15.0 \\
\hline $\begin{array}{l}\text { Mild hyperglycaemic } \\
\text { 5.9-11 mmol/dl }\end{array}$ & 268 & 41.4 & 240 & 37.0 & 183 & 28.2 \\
\hline $\begin{array}{l}\text { Moderate } \\
\text { hyperglycaemic 12-19 } \\
\text { mmol/dl }\end{array}$ & 144 & 22.2 & 116 & 17.9 & 81 & 12.5 \\
\hline $\begin{array}{l}\text { Sever } \\
\text { hyperglycaemic> 20 } \\
\text { mmol/dl }\end{array}$ & 51 & 7.9 & 29 & 4.5 & 21 & 3.2 \\
\hline Problem laboratory & 1 & .2 & 12 & 1.9 & 29 & 4.5 \\
\hline Not done & 10 & 1.5 & 99 & 15.3 & 202 & 31.2 \\
\hline Total & 646 & 99.7 & 646 & 99.7 & 646 & 99.7 \\
\hline Missing & 2 & .3 & 2 & .3 & 2 & .3 \\
\hline Total & 648 & 100.0 & 648 & 100.0 & 648 & 100.0 \\
\hline
\end{tabular}

\section{Effect of education of diabetes on control of Blood.Sugar}

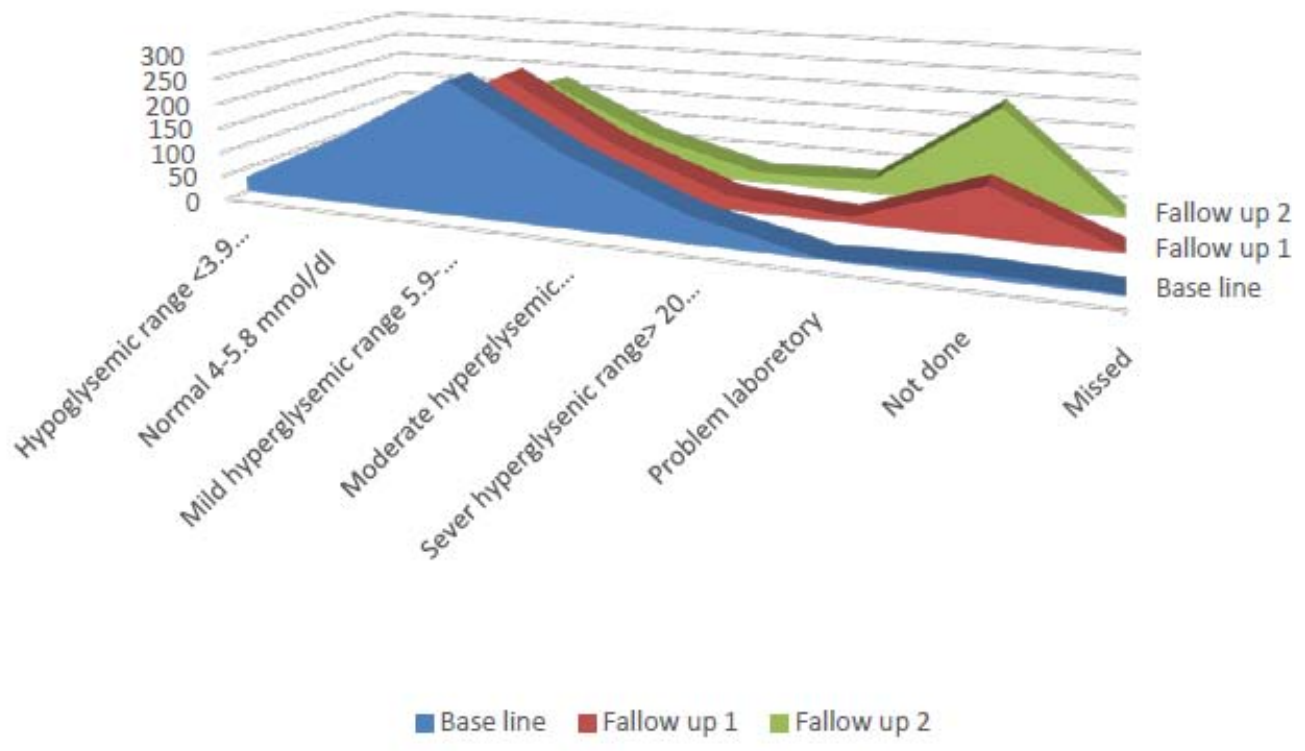

Figures 6. Linear chart: In the above charts we see the distribution of comparing fasting blood sugar scores at baseline, first follows up, and second following up after diabetes education among participants. 
Texila International Journal of Clinical Research

Volume 3, Issue 2, Dec 2016

\section{Summary table of descriptive statistics mean and standard deviation}

A sample of 648 patients with diabetes mellitus, who had participated in educational sessions regarding diabetes, was taken for study in order to determine the effect of education on improving outcomes of diabetes. The mean and standard deviation was calculated using to plan to code, without grouping into categories. For the evaluation of basic knowledge regarding diabetes mellitus prior to participating in the baseline education sessions, and assessment was performed of the pretest score. The average was $2.33(\mathrm{SD}=2.11)$. For the evaluation of knowledge gained after completing the second following up session of diabetes education, the assessment revealed an average posttest score of 7.34 $(\mathrm{SD}=1.43)$. The average age of participants were 49.79 years ( $\mathrm{SD}=13.06$ years). The average body mass index (BMI) of the participants at baseline was $26.89 \mathrm{~kg} / \mathrm{Miter}$ (SD=5.69 kg/Miter2), followed by an average BMI at the first follows up of $26.43 \mathrm{~kg} /$ Miter2 (SD=5.35 kg/Miter2), and an average $\mathrm{BMI}$ at the second following up of $26.18 \mathrm{~kg} / \mathrm{Miter} 2$ (SD=5.03 kg/Miter2). This showed that a very small decrease in BMI from baseline to the second follows up occurred. The average fasting blood glucose concentration on baseline was $10.15 \mathrm{mmol} / \mathrm{dl}(\mathrm{SD}=6.00 \mathrm{mmol} / \mathrm{dl})$, at the first follows up 9.32 $\mathrm{mmol} / \mathrm{dl}(\mathrm{SD}=5.17 \mathrm{mmol} / \mathrm{dl})$, and at the second following up $8.89 \mathrm{mmol} / \mathrm{dl}(\mathrm{SD}=5.19 \mathrm{mmol} / \mathrm{dl})$. Therefore, a gradual decrease in the fasting blood glucose concentration was observed from baseline to the second following up.

Table 5. Descriptive statistics mean and standard deviation

\begin{tabular}{|l|l|l|l|l|}
\hline & Mean & Std. Deviation & t & Asymp. Sig P \\
\hline Pretest_Score & $\mathbf{2 . 3 3}$ & $\mathbf{2 . 1 1}$ & $\mathbf{t ~ ( 2 4 . 3 3 )}$ & $\mathbf{P}<=.001$ \\
\hline Posttest_Score & $\mathbf{7 . 3 4}$ & $\mathbf{1 . 4 3}$ & $\mathbf{t ~ ( 1 1 3 . 3 6 )}$ & $\mathbf{P}<=.001$ \\
\hline Body mass index base line & 26.89 & 5.69 & & \\
\hline Body mass index first follow up & 26.43 & 5.35 & & \\
\hline $\begin{array}{l}\text { Body mass index second follow } \\
\text { up }\end{array}$ & 26.18 & 5.03 & & \\
\hline Blood glucose base line & $\mathbf{1 0 . 1 5}$ & $\mathbf{6 . 0 0}$ & $\mathbf{t ~ ( 4 2 . 5 9 )}$ & $\mathbf{P}<=.001$ \\
\hline Blood glucose first follow up & $\mathbf{9 . 3 2 9 4}$ & $\mathbf{5 . 1 7}$ & $\mathbf{t ~ ( 4 1 . 9 2 )}$ & $\mathbf{P}<=.001$ \\
\hline Blood glucose second follow up & $\mathbf{8 . 8 9}$ & $\mathbf{5 . 1 9}$ & $\mathbf{t ~ ( 3 5 . 1 1 )}$ & $\mathbf{P}<=.001$ \\
\hline
\end{tabular}

\section{Chi-squared tests}

Chi-squared tests were performed in order to determine the effect of education on patients with diabetes mellitus regarding controlling various parameters of health and diabetic complications, the knowledge gained concerning diabetes and factors involved in diabetes control, and the relationship between these effects.

\section{Chi-squared correlation between ages with other variables}

1.Correlation between ages with BMI. The results of the chi-squared test were significant, with the following associations observed: age correlated with $\mathrm{BMI}$ at baseline (value $=172.04^{\mathrm{a}}, \mathrm{df}=48, \mathrm{P}<$ .001 ), at the first follows up (value $=136.10^{\mathrm{a}}, \mathrm{df}=48, \mathrm{P}<.001$ ) and at the second following up (value $140.52^{\mathrm{a}}$, df $=48, \mathrm{P}<.001$ ).

2. Correlation between ages with fasting blood glucose concentration. The results of the chisquared test were significant, with the following associations observed: age correlated with fasting blood glucose concentrations on baseline (value $=56.69^{\mathrm{a}}, \mathrm{df}=36, \mathrm{P}<.001$ ), at the first follows up (value $=103.52^{\mathrm{a}}$, $\mathrm{df}=36, \mathrm{P}<.001$ ) and at the second following up (value $=73.06^{\mathrm{a}}$, $\mathrm{df}=36, \mathrm{P}<.001$ ). 
Table 6. Descriptive Chi-Square Tests among variables

\begin{tabular}{|c|c|c|c|}
\hline \multicolumn{4}{|l|}{ Chi-Square Tests } \\
\hline & Value & $\mathrm{df}$ & $\begin{array}{l}\text { Asymp. Sig. (2- } \\
\text { sided) }\end{array}$ \\
\hline Age * Body mass index on base line & $172.04 \mathrm{a}$ & 48 & .001 \\
\hline Age* Body mass index on first following up & $136.10^{\mathrm{a}}$ & 48 & .001 \\
\hline Age* Body mass index on seconds following up & $140.52 \mathrm{a}$ & 48 & .001 \\
\hline Age* Fasting blood sugar on base line & $56.69 a$ & 36 & .015 \\
\hline Age* Fasting blood sugar on first following up & $103.52 \mathrm{a}$ & 36 & .001 \\
\hline Age * Fasting blood sugar on seconds following up & 73.06a & 36 & .001 \\
\hline Age* Pre-test & $29.07 \mathrm{a}$ & 24 & .217 \\
\hline Age* Post-test $^{2}$ & 33.73a & 24 & .089 \\
\hline Post-test* Age & $33.73^{\mathrm{a}}$ & 24 & .089 \\
\hline Post-test $*$ Body mass index on base line & $31.62 a$ & 32 & .485 \\
\hline Post-test* Body mass index on first following up & 59.69a & 32 & .002 \\
\hline Post-test * Body mass index on seconds following up & $74.99 a$ & 32 & .001 \\
\hline Post-test $*$ Fasting blood sugar on base line & 35.13a & 24 & .066 \\
\hline Post-test * Fasting blood sugar on first following up & $50.60 \mathrm{a}$ & 24 & .001 \\
\hline $\begin{array}{l}\text { Post-test* Fasting blood sugar on seconds following } \\
\text { up }\end{array}$ & $88.02 a$ & 24 & .001 \\
\hline
\end{tabular}

\section{Pearson correlation}

In order to examine the effect of education regarding diabetes mellitus on controlling various variables of health and the control of diabetic complications, and to determine the amount of knowledge gained after diabetes education, a Pearson correlation matrix was created using all variables in order to determine the relationship between them.

\begin{tabular}{|c|c|}
\hline $\begin{array}{c}\text { Age with body mass index of baseline, first } \\
\text { follow up and second follow up }\end{array}$ & $(\mathrm{P}<.001)$ \\
\hline $\begin{array}{c}\text { Age with blood sugar of baseline, first follow } \\
\text { up and second follow up }\end{array}$ & $(\mathrm{P}<.001)$ \\
\hline $\begin{array}{c}\text { Posttest with body mass index of baseline, first } \\
\text { follow up and second follow up }\end{array}$ & $(\mathrm{P}<.01)$ \\
\hline $\begin{array}{c}\text { Posttest scores with fasting blood sugar of } \\
\text { baseline, first follow up and second follow up }\end{array}$ & $(\mathrm{P}<.01)$ \\
\hline $\begin{array}{c}\text { Age was significantly positively correlated } \\
\text { with pretest and posttest scores }\end{array}$ & $(\mathrm{P}=.01)$ \\
\hline
\end{tabular}

\section{ANOVA and regression analysis}

In order to examine the effect of diabetes education on the control of diabetes and on the incidence of complications, a multiple linear regression was conducted. This allowed an assessment of the control of fasting blood sugar concentrations on baseline as a dependent variable, with the independent variables of baseline, BMI, and post-test score.

Table 8. Regression, ANOVA analysis and post hoc Turkey test:

Base line

linear regression

The individual predictor

Post hoc Turkey tests
Significant $\mathrm{F}(7,637)=2.44, \mathrm{P}<=.018, \mathrm{R} 2=$ .026

$\mathrm{B}=1.94 \mathrm{P}<.001$ suggest that for every one unit increase in baseline fasting blood sugar, the other variables baseline body mass index, 


\begin{tabular}{|c|c|}
\hline & $\begin{array}{l}\text { increase } 1.94 \text { Unit, } \\
\text { Baseline body mass index, compared with } \\
\text { dependent variable baseline follow up fasting } \\
\text { blood sugar significant } \mathrm{P}<.05 \text {. }\end{array}$ \\
\hline $\begin{array}{l}\text { First follow up } \\
\text { linear regression } \\
\text { The individual predictor } \\
\text { Post hoc Turkey tests }\end{array}$ & $\begin{array}{l}\text { significant, } \mathrm{F}(7, .673)=52.34, \mathrm{P}<=.001, \mathrm{R} 2= \\
.365 \mathrm{R} 2=.365 \\
\mathrm{~B}=0.42 \mathrm{P}<.001 \text { suggests that for every one unit } \\
\text { increase in first following up fasting blood } \\
\text { sugar, the other variables in first following up } \\
\text { body mass index, increase } 0.42 \text { Unit, } \\
\text { Body mass index, compared with dependent } \\
\text { variable in first follows up blood sugar are } \\
\text { significant } \mathrm{P}<.005 \text {. }\end{array}$ \\
\hline $\begin{array}{l}\text { First follow up } \\
\text { linear regression } \\
\text { The individual predictor } \\
\text { Post hoc Turkey tests }\end{array}$ & $\begin{array}{l}\text { significant, } \mathrm{F}(7, .673)=52.34, \mathrm{P}<=.001, \mathrm{R} 2= \\
.365 \mathrm{R} 2=.365 \\
\mathrm{~B}=-.165 \mathrm{P}<.001 \text { suggests that for every one unit } \\
\text { decrease in seconds following up fasting blood } \\
\text { sugar, the other variables second follow up body } \\
\text { mass index, decrease }-.165 \text { Unit } \\
\text { Body mass index, compared with dependent } \\
\text { variable in first following up blood sugar are } \\
\text { significant } \mathrm{P}<.005 \text {. }\end{array}$ \\
\hline $\begin{array}{l}\text { Second follow up } \\
\text { linear regression } \\
\text { The individual predictor } \\
\text { Post hoc Turkey tests }\end{array}$ & $\begin{array}{l}\text { significant, } \mathrm{F}(8,632)=66.37, \mathrm{P}<=.001, \mathrm{R} 2= \\
.450 \\
\mathrm{~B}=0.42 \mathrm{P}<.001 \text { suggests that for every one unit } \\
\text { increase in first following up fasting blood } \\
\text { sugar, the other variables first follow up body } \\
\text { mass index, increase from } 0.42 \text { Unit, } \\
\text { Second following up body mass index, } \\
\text { compared with dependent variable seconds } \\
\text { following up fasting blood sugar are significant } \\
\mathrm{P}<.005 \text {. }\end{array}$ \\
\hline
\end{tabular}

\section{Discussion}

The present study found that educational intervention were highly effective against controlling anthropometric parameters (BMI), as these had significantly decreased in baseline at the second patient follows up sessions. Metabolic control (blood sugar) also showed a significant positive improvement in baseline at the second follows up visit. Finally, participants also showed an improvement in knowledge through diabetes education. This was assessed by a pre-test prior to the commencement of education and a post-test after the completion of second following up educational sessions at the central hospital in Nampula. The knowledge provided by the education helped the participants to improve and change their lifestyle, especially their dietary and exercise habits, their psychological adjustment and their attitude to living with diabetes.

Similar studies have previously been performed, with some comparable findings being reported. Newly diagnosed diabetic patients need self-management education, as this helps to increase their level of knowledge of diabetes and to provide them with skills to manage their diabetes life long, as it is a chronic condition (American Diabetes Association, 2014). A Cochrane review (Deakin, 2009) concluded that a reduction in blood sugars concentrations, reduced HbA1c, reduced BMI, reduced systolic and increased knowledge of diabetes. A Cochrane review (Deakin, 2009) concluded that from 
group education of diabetes patients get motivated, start adherence to treatment and understand diabetes. The study performed by Balagopal et al. (2008) showed reduced levels of obesity and improved dietary habits of diabetic and pre-diabetic patients after education regarding diabetes. The lifestyle modifications were effective against reducing a number of the risk factors of type 2 diabetes mellitus and in improving self-management of the disease. Meta analyses and the outcome of various studies has shown positive impacts after receiving diabetes education, and enhanced knowledge of diabetes have been presented by Ricci-Cabello et al. (2014). In order to promote diabetes awareness, self-care behaviors can be useful. Choi et al. (2016) described innovative strategies for the improvement on diabetic control and glycemic improvement in Chinese patients through the continuing education of diabetes mellitus during patient examination and by increasing family involvement via diabetic knowledge. Mollaoğlu et al. (2009) emphasized repeated diabetic education sessions to control and improve metabolic parameters. Salinero-Fort et al. (2011), using experimental and control groups regarding diabetes education.

The present study found that participants had age group of diabetes mellitus were 41-60 years, of which $56 \%$ were male. A further study found that, regarding type of diabetes, diabetes mellitus type 2 was detected at the highest prevalence of $87.5 \%$, however the prevalence of diabetes mellitus type 1 was $5.1 \%$.

\section{Conclusion}

The Mozambique, which shows a progressive increase in the number of patients with diabetes mellitus due to the lack of a healthy diet, a sedentary life style and urbanization. Regarding patients with diabetes mellitus in a central hospital in Nampula, it was recognized that there was a need to organized education concerning diabetes, such as regarding the diabetic diet, increasing patient knowledge of diabetes to avoid the risks of complications, physical activity and its importance, and the psychological motivation to live with diabetes.

The study also showed that the majority of the participants had diabetes mellitus types 2, were in the majority of the patients controlled their fasting blood glucose, body mass index and increased knowledge of diabetes from education of diabetes.

\section{Motivational quotes}

"Exercise and diet can help prevent or even totally reverse metabolic conditions like diabetes and cardiovascular disease - only thing is, you've got to catch them young... You know, while these conditions are still of 'impressionable minds'!"

\section{- Deepak 'The Fitness Doc' Hiwale}

There is currently a need to design a national policy and program for diabetes education. Clinicians and health educators should continue to reemphasize that patients with diabetes mellitus make healthy behavioral changes in order to control their diabetes and reduce the occurrence of complications.

The limitations of this study include that some of the diabetic patients used traditional medications, some did not adhere to treatment, and some were lost to follow up, all of which can cause uncontrollable diabetes and increase the incidence of complications. Patients were very interested in taking medicine free of charge in a government hospital. Limitations were also found here, such as the intermittent non-availability of the results of blood sugar results and other biochemical results due to a lack of laboratory reagents. Patients who lived district, distance from the hospital, were not able to regularly attend three education sessions. Patients were more interested in obtaining medication than on lifestyle modification. Patients were generally from a poor or lower income group, and were unable to buy the recommended food. It was also noted that some patients had an insufficient economic condition to take small and frequent meals. Some of the patients presented with a delayed diagnosis, with irreversible complications.

One of the strengths of this study is that patients, at the commencement of educational sessions, were encouraged to participate and to bring laboratory results and other activities to the follow up sessions by reminding them that they would receive prescription medicine at the end of the successful 
completion of all of the essential activities involved in the education sessions. This encouraged patients to take a further interest in the study, and the majority of these patients then implemented the required changes in their lives and achieved positive significant outcomes of controlling their diabetes.

\section{Contribution to knowledge}

This study adds to the current body of knowledge regarding lifestyle modification and patient knowledge of diabetes. The education provided with this study allowed patients to understand diabetes and to control and minimize related complications.

\section{Suggestion for future research}

However, a need remains to involve other departments in future study, for example emergency and intensive care medicine, district hospitals and effect of education of diabetes of family members to control the diabetic of patient, in order to see effect of education of diabetes to improve the knowledge of diabetes in public for primary prevention of diabetes in society.

\section{References}

[1]. Association, A. D. (2002). Standards of medical care for patients with diabetes Mellitus. Diabetes Care, 25(suppl 1), 33-49. doi:10.2337/diacare.25.2007.S33In-line Citation :( Association, 2002)

[2]. Balagopal, P., Kamalamma, N., Patel, T.G. and Misra, R. (2008) 'A community-based diabetes prevention and management education program in a rural village in India', Clinical Care/Education/Nutrition/Psychosocial Research, 31 (6), pp. 1097-1104. doi: 10.2337/dc07-1680. In-text citations: (Balagopal et al., 2008)

[3]. Choi, T.S.T., Davidson, Z.E., Walker, K.Z., Lee, J.H. and Palermo, C. (2016) 'Diabetes education for Chinese adults with type 2 diabetes: A systematic review and meta-analysis of the effect on glycemic control', Diabetes Research and Clinical Practice, 116, pp. 218-229. doi: 10.1016/j.diabres.2016.04.001. In-text citations: (Choi et al., 2016)

[4]. Disclaimer, I. D. F. (2015). Mozambique. Retrieved August 29, 2016, from http://www.idf.org/membership/afr/mozambiqueIn-line Citation:(Disclaimer, 2015)

[5]. 5.5 general diabetes self-management and education (2016) Available at: https://www.icsi.org/guideline_sub-pages/diabetes/55_general_diabetes_self-management_and_education/ (Accessed: 14 August 2016). In-text citations: (5.5 general diabetes self-management and education, 2016) [6]. Mollaoğlu, M. and Beyazıt, E. (2009) 'Influence of diabetic education on patient metabolic control', Applied Nursing Research, 22 (3), pp. 183-190. doi: 10.1016/j.apnr.2007.12.003. In-text citations: (Mollaoğlu and Beyazit, 2009)

[7]. Moattari, M., Ghobadi, A., Beigi, P. and Pishdad, G. (2012) 'Impact of self-management on metabolic control indicators of diabetes patients', Journal of Diabetes \& Metabolic Disorders, 11 (1), p. 6. doi: 10.1186/2251-6581-11-6. In-text citations: (Moattari et al., 2012)

[8]. Ricci-Cabello, I., Ruiz-Pérez, I., Rojas-García, A., Pastor, G., Rodríguez-Barranco, M. and Gonçalves, D.C. (2014) 'Characteristics and effectiveness of diabetes self-management educational programs targeted to racial/ethnic minority groups: A systematic review, meta-analysis and meta-regression', BMC Endocrine Disorders, 14 (1), p. 60. doi: 10.1186/1472-6823-14-60. In-text citations (Ricci et al., 2014)

[9]. Salinero-Fort, M., Santa, C., Arrieta-Blanco, F., Abanades-Herranz, J., Martín-Madrazo, C., RodésSoldevila, B. and Burgos-Lunar, de (2011) 'Effectiveness of PRECEDE model for health education on changes and level of control of HbA1c, blood pressure, lipids, and body mass index in patients with type 2 diabetes mellitus', BMC public health., 11. In-text citations: (Salinero-Fort et al., 2011) 


\title{
Mitigating Lung Infections in Poor HIV Patients
}

\author{
Article by Lois Collie-Acasio \\ Ph.D. Clinical Research Texila American University \\ Email: licollie@yahoo.com
}

\begin{abstract}
Critical Appraisal of head-to-head studies is an important decision-making skill. Questions followed by evidence statements are responses that give researchers the evidence-based information they need to make decisions on the best treatment for an indication. The lung is the organ that HIV most frequently infects, and respiratory complications, such as pneumonia, are a common cause of death. The question of interest is: "Which of two treatments is the best one to mitigate lung infections in poor HIV patients?" This paper is a critical appraisal done to find an antibiotic that may be best for poverty-stricken HIV patients with pneumonia. There have been many reports of substandard drugs being donated to poorer countries. Critical appraisals need to be done on certain drugs that are donated to poverty-stricken countries to see if the drug is cost-effective, useful and affordable. It should be unethical to donate useless drugs to vulnerable poor HIV patients with lung infections.
\end{abstract}

Keywords: Critical appraisal, Decision-making, Randomized Controlled Trial (RCT) Human Immunodeficiency Virus (HIV)

\section{Introduction}

There may be several reasons why patients in low-income countries are not able to access the lifesaving drugs that they need.

1. The cost of the best intervention may be prohibitive.

2. The drug may need to be refrigerated, but refrigerators may not be accessible in rural areas.

3. The research, development, and production of the needed drug may not be profitable.

4. A substandard or counterfeit version of the needed drug may be available.

5. Foundations may be donating expired version of the needed drug.

The first thing that needs to be done is to identify the drug that is best and then seek ways and means to bring the costs down and to make the drug available without compromising quality.

This article is a critical appraisal of a clinical trial report and has been prepared for Texila American University E-Conference Participation. It has been peer-reviewed by the student supervisor. All the evidence statements are based on the information provided in a paper by (Cordero, E., Bouza, E., Ruiz, I., Pachon, J., 2001). Cefepime versus cefotaxime for empirical treatment of bacterial pneumonia in HIV-infected patients: an open, randomized trial. The graph shows the number of daily deaths from respiratory infections and HIV/AIDs. Infectious diseases are poverty-related. (WHO, 2004):

\section{Background}

Clinical scenario: Bacterial pneumonia is a severe opportunistic infection of the respiratory tract that targets HIV-positive people about eight times more than HIV-negative people, regardless of high CD4 cell and a good response to treatment. Although the introduction of powerful antiretroviral combination therapy in the battle against HIV has caused the incidence of bacterial pneumonia to drop, bacterial pneumonia is still life threatening to patients and may be acquired as a nosocomial infection as well from the community Some of the bacteria that cause respiratory tract infections and recurrent pneumonia in HIV-positive patients are Streptococcus pneumoniae, Staphylococcus aureus, Pseudomonas aeruginosa, and Haemophilus influenza, with the first one listed being the most 
common. Bacterial pneumonia may cause death when the bacteria infection becomes systemic, and the CD 4 count falls below 100. HIV-positive people with a higher risk of getting bacterial pneumonia when their lifestyle embraces cofactors such as smoking, use of alcohol, cocaine, and intravenous drugs, and those who have cirrhosis of the liver. (Aviram Galit and Phillip M. Boiselle, 2004)

\section{Objective}

To identify the better antibiotic for these patients.

Is the question that this study addresses focused?

\section{YES}

Evidence Statement 1

The question was clearly focused and addressed patients, intervention, comparing interventions and the outcomes (PICO): The participants in the study had bacterial pneumonia and were HIV- positive. The study compared the efficacy and safety of cefepime to cefotaxime (with dosage adjustments for Pseudomonas aeruginosa pneumonia) in the empirical treatment of bacterial pneumonia in HIVinfected patients. The primary endpoint concerned the safety and efficacy of both treatments with the clinical control of the bacterial infection as the secondary endpoint.

1. Was randomization and control used in assigning the subjects to the study drugs?

YES

Evidence Statement 2

The study was appropriately randomized and controlled. The randomization process was computerized, and the randomization list was concealed. It was a multi-centred study that involved a comparison of two antibiotic study drugs, and it was randomized to prevent participants and research staff from having the bias of favoring one drug over the other.

2. Were subjects allocated in a balanced and appropriate way to both arms of the intervention?

YES

Evidence Statement 3

The study had two well-balanced arms was comprised of hospitalized patients whom all had bacterial pneumonia and HIV infection.

3. Were the researchers and subjects 'blind' to the study group assignments?

NO

Evidence Statement 4

The study was open and not blinded. It was conducted in full compliance with GCP Guidelines and the Declaration of Helsinki.

4. Were all of the participants accounted for at the end of the study?

YES

Evidence Statement 5

All the participants were hospitalized and were analyzed either on an as per protocol (PP) basis or on an intention to treat (ITT) analysis in the groups to which they were randomized. In the ITT group, any participant that was not able to complete study was still regarded as a part of the study in the final analysis.

5. Was the method of follow up and data collection the same for all the groups?

YES

Evidence Statement 6

Follow-up was only done if it was clinically necessary. There usually was no need for a follow- up because the study lasted from 5 to of 7 days with one of the endpoints being if the participant found the study drug to be intolerable. The data was collected the same way in both arms of the study. (Cordero, E., Bouza, E., Ruiz, I., Pachon, J., 2001).

6. Were enough subjects enrolled in the study to minimize the possibility of chance? YES

Evidence Statement 7 
Statistical calculations were done to find out how many participants needed to be treated in order to give the right statistical power to the study. (Guyatt G.H, Sackett D.L, Cook D.J., 1993) The researchers assumed a success rate of $80 \%$ in the sample calculation. It was estimated that each treatment arm needed at least 76 participants in order detect differences that would be equal or greater than $15 \%$ with a 2 sided probability of alpha $=0.05$ and beta $=0.20$. A type 1 error (alpha) occurs when there is a probability that the null hypothesis has been rejected even though it was true and is taken to be equal or less than $5 \% \mathrm{p}<0.05$. A Type II error (beta) occurs when there is a probability that the null hypothesis is true when it is false, and this is taken to be equal to 0.20 when the power is from 80 to $90 \%$. The result of the power analysis revealed that the sample size of 150 and 160 per treatment arm was adequate to reveal any differences. As a result 76 subjects were recruited for the cefotaxime arm, 73 of which completed the study, and 84 subjects were recruited for the cefepime arm with 77 of them completing the study.

7. How were the results presented and expressed? What were the main results and the size of the treatment effect?

Evidence Statement 8

Data were presented in several tables that showed the main result was positive. The size of the treatment effect was significantly high, and the results were clearly expressed. The researchers used $\mathrm{x} 2$ tests and Fisher's exact tests to analyze the data and to make the comparisons. In order to determine factors that contributed to any failure of the treatment, the researchers used logistic regression analysis. From the calculations for Relative Risks and Relative Risks Reduction that follow in Tables 1, a, b, c, and d, it may be shown that no significant difference was observed in the way subjects tolerated both study drugs neither in the efficacy and clinical endpoints of both antibiotics.

\section{Results}

Table 1 a. Calculations

\begin{tabular}{|l|l|l|l|}
\hline As Per Protocol (PP) & \multicolumn{2}{|l|}{$\begin{array}{l}\text { Outcome event } \\
\text { Primary Endpoint: Tolerance } \\
\text { after 3-5 days }\end{array}$} & \\
\hline Group n=150 & Yes & No & Total \\
\hline Experimental group & a & b & a + b \\
\hline Cefepime 84 & $72(93.5 \%)$ & $5(6.5 \%)$ & 77 \\
\hline Control group & c & d & c + d \\
\hline Cefotaxime 76 & $59(80.8 \%)$ & $14(19.1 \%)$ & 73 \\
\hline
\end{tabular}

Experimental event rate $=$ risk of outcome event in experimental group $=$ EER $=\mathrm{a} /(\mathrm{a}+\mathrm{b})$

$=72 / 77$

$=0.93$

Control event rate $=$ risk of outcome event in control group $=$ CER $=c /(c+d)$

$=59 / 73$

$=0.80$

Relative risk $(\mathrm{RR})=\mathrm{EER} / \mathrm{CER}=0.93 / 0.80=1.16$ 
Texila International Journal of Clinical Research

Volume 3, Issue 2, Dec 2016

Table 1 b. Calculations

\begin{tabular}{|l|l|l|l|}
\hline Intention-to-treat (ITT) & \multicolumn{2}{|l|}{$\begin{array}{l}\text { Outcome event } \\
\text { Primary Endpoint: Tolerance }\end{array}$} & \\
& after 3-5 days & \\
\hline Group n=160 & Yes & No & Total \\
\hline Experimental group & a & b & a + b \\
Cefepime 84 & $72(85.7 \%)$ & $12(14.3 \%)$ & 84 \\
\hline Control group & $\mathrm{c}$ & $\mathrm{d}$ & $\mathrm{c}+\mathrm{d}$ \\
\hline Cefotaxime 76 & $59(77.6 \%)$ & $17(22.4 \%)$ & 76 \\
\hline
\end{tabular}

Experimental event rate $=$ risk of outcome event in experimental group $=$ EER $=a /(a+b)$

$=72 / 84$

$=0.857$

Control event rate $=$ risk of outcome event in control group $=\mathrm{CER}=\mathrm{c} /(\mathrm{c}+\mathrm{d})$

$=59 / 76$

$=0.77$

Relative risk $(\mathrm{RR})=\mathrm{EER} / \mathrm{CER}=0.857 / 0.77=1.11$

Table 1 c. Calculations

\begin{tabular}{|c|c|c|c|}
\hline As Per Protocol (PP) & \multicolumn{2}{|c|}{$\begin{array}{l}\text { Outcome event } \\
\text { Clinical Response to } \\
\text { antibiotics EOT }\end{array}$} & \\
\hline Group n=150 & Yes & No & Total \\
\hline $\begin{array}{l}\text { Experimental group } \\
\text { Cefepime } 84\end{array}$ & $\begin{array}{l}\text { a } \\
50(65.8 \%)\end{array}$ & $\begin{array}{l}\mathrm{b} \\
13(17.1 \%)\end{array}$ & $\begin{array}{l}a+b \\
63\end{array}$ \\
\hline Control group & C & $\mathrm{d}$ & $c+d$ \\
\hline Cefotaxime 76 & 50 (68.5\%) & $10(13.7 \%)$ & 60 \\
\hline
\end{tabular}

Experimental event rate $=$ risk of outcome event in the experimental group $=\mathrm{EER}=\mathrm{a} /(\mathrm{a}+\mathrm{b})$

$=50 / 63$

$=0.79$

Control event rate $=$ risk of outcome event in the control group $=\mathrm{CER}=\mathrm{c} /(\mathrm{c}+\mathrm{d})$

$=50 / 60$

$=0.83$

Relative risk $(\mathrm{RR})=\mathrm{EER} / \mathrm{CER}=0.79 / 0.83=1.25$

Relative risk reduction $(\mathrm{RRR})=(\mathrm{CER}-\mathrm{EER}) / \mathrm{CER}$

$(0.83-0.79) / 0.83=0.04 / 0.83$

$=0.04$ or $4 \%$

The bacterial infection may be reduced by $4 \%$ more in the treatment group than in the control group which is a comparatively small percentage of a difference between treatments.

Table $1 \mathrm{~d}$. Calculations

\begin{tabular}{|l|l|l|l|}
\hline Intention-to-treat (ITT) & \multicolumn{2}{|l|}{$\begin{array}{l}\text { Outcome event } \\
\text { Clinical Response to }\end{array}$} & \\
& antibiotics EOT & \\
\hline Group n=160 & Yes & No & Total \\
\hline Experimental group & a & b & a + b \\
\hline Cefepime 84 & $59(70.2 \%)$ & $14(16.7 \%)$ & 63 \\
\hline Control group & c & d & c + d \\
\hline Cefotaxime 76 & $59(77.6 \%)$ & $6(7.9 \%)$ & 65 \\
\hline
\end{tabular}


Experimental event rate $=$ risk of outcome event in the experimental group $=$ EER $=a /(a+b)$

$=59 / 63$

$=0.79$

Control event rate $=$ risk of outcome event in the control group $=\mathrm{CER}=\mathrm{c} /(\mathrm{c}+\mathrm{d})$

$=59 / 65$

$=0.91$

Relative risk $(\mathrm{RR})=\mathrm{EER} / \mathrm{CER}=0.93 / 0.91=1.02$

8. Were the results precise and presented with confidence intervals?

YES

Evidence Statement 9

Yes, CI data and p-values were presented on pages 429, 530 and 531 and the results were precise enough to show that both drugs were equally tolerated by the participants. This finding was supported by a similar study done with subjects that did not have HIV (Edelstein, H., Chirurgi, V., Oster, S., Karp, R., Cassano, K., Aiken, S., 1991). In the similar study of the effect that cephalosporin drugs have on bacterial pneumonia, the patients were not HIV compromised, and the study drugs were cefepime versus cefotaxime (NOT ceftazidime which has a similar name and is very similar in formulation and properties).

9. Were the important outcomes and results applicable to society?

YES

Evidence Statement 10

Yes. Although it did not reveal how many patients suffering from liver damage were in each arm of the study, all patients were given a creatine serum test, to check for liver damage. Exactly $64.3 \%$ of the participants had abused drugs, 56.2\% of them had a hepatobiliary disease, $18.7 \%$ abused alcohol and $81.8 \%$ were cigarette smokers. The dosages of medicines that are administered to HIV-positive patients should be calculated to go easy on their liver as it is highly probably that liver damage may be a co-morbid condition.

Many HIV Patients suffer from the end-stage of liver disease. Concerns about the high incidence of liver damage in HIV-positive patients have been expressed in several publications, and the administration of HAART drugs only makes the level of liver damage higher. Therefore, any drug intervention to an HIV patient must consider the additional damage that that drug may do the liver. (Crowe, David, 2011)

\section{Discussion}

\section{Summary of best evidence}

Table 2: Description and appraisal of Cefepime versus cefotaxime for empirical treatment of bacterial pneumonia in HIV-infected patients: an open, randomized trial, (Cordero, E., Bouza, E., Ruiz, I., Pachon, J., 2001)

\section{The objective of the study}

Study Design: The study was open and randomized by computer. The allocations were sealed, so the study was blinded. The outcomes measured how well patients tolerated the treatments, and how successful the antibiotics were in removing the bacterial infection.

Setting: This was a multi-centred and the subjects were hospitalized in eighteen (18) clinics in Spain.

\section{Participants}

Diagnosis: Bacterial pneumonia and HIV infected.

Inclusion criteria: participant must be 18 years old and above, male or female, must be HIV infected, with confirmed case of bacterial pneumonia, 
Texila International Journal of Clinical Research

Volume 3, Issue 2, Dec 2016

Exclusion criteria: allergic to cephalosporins, pregnancy, breastfeeding, previously enrolled in this study, a life expectancy of fewer than 60 days, or evidence of serum creatine of $2 \mathrm{mg} / \mathrm{dL}$ which shows severe liver damage.

The patient was recruited in emergency room settings, signed the informed consent form and then randomized on a 1:1 basis. The number of drop-outs did not affect the ITT or PP study and was minimal because the study lasted for no more than 7 days. Follow-up was done at the discretion of the clinician if needed.

Comparator Drug Arm: Cefotaxime. Assigned Drug Intervention: Cefotaxime $2 \mathrm{gm}$ IV tid

Comparator Drug Arm: Cefepime Assigned Drug Intervention: Cefepime $2 \mathrm{gm}$ IV bid $(2 \mathrm{~g}$ tid daily in case of Pseudomonas aeruginosa pneumonia)

\section{Intervention investigated}

To compare the tolerability and efficacy of cefepime versus cefotaxime: Open and blind multicentre study of hospitalized HIV-positive patients with HIV infection, $>18$ years of age with bacterial pneumonia. Patients were randomized to cefepime or cefotaxime 2 grams bid and tid respectively to be administered intravenously. Patients who responded could be switched to oral therapy.

Main Outcome Measures: Intolerance to the treatment and clinical success of the antibiotic therapy. Cefotaxime cleared the bacterial infection in 93.4\%, and Cefepime cleared it in $100 \%$ of the subjects. Patients had approximately the same amount of intolerance to both drugs.

\section{Critical appraisal}

\section{Validity}

Critical appraisal revealed that the clinical trial was properly conducted and that all the Critical points were properly addressed. All of the questions were answered positively.

The PEDro scales also did not find any fault with the design of the study. (PEDro, 1999)

\section{Area of Concern}

Enzyme induction is when drugs increase the liver enzymes and cause the rate of metabolism

to increase. Cigarette smoking, alcohol, and drug abuse may induce enzyme induction because of liver disease. These interactions are difficult to predict, and the patient may find it difficult to tolerate the study drug. Because of these concerns, the study has made tolerance of the drug its primary outcome.

\section{Interpretation of results}

The outcomes were favorable. The alpha level of probability was estimated to be $5 \%$ ( $\mathrm{p}$ - value $<0.05$ ), so it is statistically significant and not likely due to chance. CI scores were provided.

\section{Results}

Critical Appraisal of the meta-analysis provided evidence of the effectiveness of various forms of NRT smoking cessation interventions and justified their high cost. Critical Appraisal also showed that the two antibiotics in the head-to-head study, Cefepime, and Cefotaxime, were equivalent in effectiveness and tolerability. It was shown that the results of the both studies were valid and not due to chance. It has been shown that Cefepime antibiotics have the lowest average cost per patient. (Halpern, M., Brown, R., Drolet, M., Sorensen, S., Mandell, L., 1997)

\section{Conclusion}

Critical Appraisal identifies evidence-based interventions. Respiratory infections and HIV/AIDS are poverty-related communicable diseases with high mortality. (WHO, 2004) The risks of morbidity and mortality need to be reduced. Patients need to maintain an optimal QOL despite of opportunistic diseases. More critical appraisals are needed to identify safe, effective, and affordable interventions 
for poverty-stricken and unemployed patients. The economically disadvantaged HIV patients are vulnerable, and it should be unethical to give them watered-down HIV/AIDS drugs. Donations of such drugs need to be refused by the Public Health Departments of the countries concerned.

\section{Appendix: key formulas}

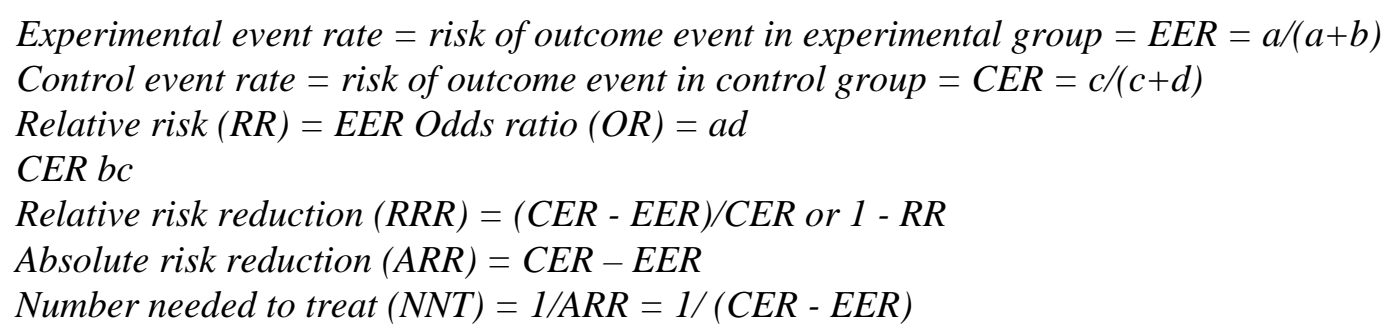

\section{References}

[1]. Aviram Galit and Phillip M. Boiselle (2004) Imaging Features of Bacterial Respiratory Infections in AIDS: Current Opinion Pulmonary Medicine, 10(3) [Online] Available at http://www.medscape.com/viewarticle/475231_2 (Accessed 15 September 2016)

[2]. Cordero, E., Bouza, E., Ruiz, I., Pachon, J. (2001). Cefepime versus cefotaxime for empirical treatment of bacterial pneumonia in HIV-infected patients: an open, randomized trial. J Antimicrob Chemother 48: 527-534 [Online] Available at http://jac.oxfordjournals.org/content/48/4/527 (Accessed 15 September, 2016)

[3]. CASP, (2006) Guyatt G H, Sackett D L, and Cook D J, Users' guides to the medical literature. II. How to use an article about therapy or prevention. Critical Appraisal Skills Programme, JAMA 1993; 270 (21): 25982601 and JAMA 1994; 271(1): 59-63 [Online] Available at http://www.sph.nhs.uk/sph-files/casp-appraisaltools/rct\%20appraisal\%20tool.pdf

[4]. (Accessed 15 September, 2016)

[5]. Crowe, David, (2011) Liver Damage, Concerns about HAART (Highly Active Anti-Retroviral Therapy), Alberta Reappraising AIDS Society [Online] Available at http://aras.ab.ca/haart-liver.html (Accessed 15 September, 2016)

[6]. Edelstein H, Chirurgi V, Oster S, Karp R, Cassano K, Aiken S, McCabe R(1991) A randomized trial of cefepime (BMY-28142) and ceftazidime for the treatment of pneumonia. J Antimicrob Chemotherapy,28(4):569-75.[Online] Available at

[7]. http://www.druglib.com/abstract/ed/edelstein-h_j-antimicrob-chemother_19911000.html (Accessed 15 September, 2016)

[8]. FDA, (2009) Maxipime (cefepime hydrochloride), Statistical Review and Evaluation, [Online] Available at

[9]. http://www.fda.gov/downloads/Drugs/DrugSafety/PostmarketDrugSafetyInformationforPatientsandProvid ers/DrugSafetyInformationforHeathcareProfessionals/UCM167309.pdf (Accessed 26 September, 2016)

[10]. Guyatt GH, Sackett D.L, Cook D.J., (1993) Users' guides to the medical literature. II.

[11]. How to use an article about therapy or prevention, A. Are the results of the study valid? JAMA 1993; (270): 2598-2601.,

[12]. [Online] Available at http://www.cche.net/text/usersguides/therapy.asp (Accessed 15 September, 2016)

[13]. Halpern, M., Brown, R., Drolet, M., Sorensen, S., Mandell, L., (1997) Decision analysis modelling of costs and outcomes following cefepime monotherapy in Canada, The Can J Infect Dis.1997 Jan-Feb; 8(1): 1927.[Online\} Available at http://www.ncbi.nlm.nih.gov/pmc/articles/PMC3327332/ (Accessed 16 September, 2016)

[14]. PEDro scale, (1999) Physiotherapy Evidence Data Base [Online] Available at http://www.pedro.org.au/english/downloads/pedro-scale/ (Accessed 15 September, 2016)

[15]. SIGN (2011) Methodology Checklist w: randomised controlled trials, Scottish intercollegiate guidelines Network, [Online] Available at http://www.sign.ac.uk/guidelines/fulltext/50/checklist2.html (Accessed 15 September, 2016) 
Texila International Journal of Clinical Research

Volume 3, Issue 2, Dec 2016

[16]. Silagy, C., Mant D, Fowler G, Lodge M., (1994) Meta-analysis on efficacy of nicotine replacement therapies in smoking cessation, The Lancet, 343, 8890 p. 139 [Online] Available at http://www.ncbi.nlm.nih.gov/pubmed/7904003 (Accessed 15 September, 2016)

[17]. SFAF, (2011) Syringe Access, San Francisco Aids Foundation, [Online] Available at http://www.sfaf.org/client-services/health-services/syringe-access/ (Accessed 15 September, 2016)

[18]. Mhaskar, R., Emmanuel, P., Mishra, S., Patel, S., Naik, E., and Kumar' A.,(2009) "Critical Appraisal Skills Are Essential to Informed Decision-Making." Indian Journal of Sexually Transmitted Diseases 30.2 (2009): 112-119. PMC. [Online] Available at http://www.ncbi.nlm.nih.gov/pmc/articles/PMC3168054/ (Accessed 15 Sept. 2016).

[19]. Oxman AD, (1994) CASP, User’s Guide to Medical Literature VI, JAMA 272:1367-1371

[20]. [Online] Available at http://www.ncbi.nlm.nih.gov/pubmed/7933399 (Accessed 15 September, 2016)

[21]. WHO (2004) Disease of poverty and the 10/90 Gap, World Health Organization [Online] Available at http://www.who.int/intellectualproperty/submissions/InternationalPolicyNetwork.pdf (Accessed 16 September, 2016)

[22]. Wu, P., (2006) Effectiveness of smoking cessation therapies: a systematic review and meta-analysis, Biomed Central Journal, 6:300 [Online] Available at http://www.biomed.com/1471-2458/6/300, (Accessed 15 September, 2016) 


\title{
Prevalence and Management of Rhinosinusitis in Developing Countries: the Case of Mbingo Baptist Hospital, North West Region, Cameroon
}

\author{
Article by Tcheuwebe Liale Honore \\ $R N-B S N$, Texila American University \\ Email: honor_liale2002@yahoo.fr
}

\begin{abstract}
Rhinosinusitis occurs worldwide and affects about $16 \%$ of the adult American population annually significantly impairing their quality of life. Allergic rhinitis and chronic rhinosinusitis are prevalent non communicable diseases increasing around the world, even in Africa.

This study is aimed at assessing the prevalence and management of rhinosinusitis in Mbingo Baptist Hospital, in children, teenagers and adults. In Cameroon, the investigator did not find any readily documented and published statistics indicating the case of rhinosinusitis. In the hospital where this study was carried out, observation made in the frequency of rhinosinusitis and knowing the possible complications prompted the investigator find out about the prevalence and its management.

Thus the following research question of "what is the prevalence and management of rhinosinusitis?”

Our main objective is to assess the prevalence and management of rhinosinusitis in Mbingo Baptist Hospital.

More specifically, the investigator will be able:

- To define and describe rhinosinusitis

- To assess the management of rhinosinusitis in $M B H$

- To come out with the prevalence of rhinosinusitis in $M B H$

- To ascertain the standard management of rhinosinusitis
\end{abstract}

The prevalence of $A R$ is very high (>35\%) among Nigerian Africans, and it is likely that environmental factors are responsible for major differences with other countries. Knowing the prevalence of Rhinosinusitis will serve as reference for many other investigations. This prevalence would tend to increase if the condition is not well managed at the level of the hospital.

This is a cross sectional study in which data are collected from the hospital registers for patients having rhinosinusitis since 2011 till 2015. With these data, we aim at giving the prevalence of rhinosinusitis during this period.

The study will also include 5 case studies met in the ENT department during the period of the study both on an in-patient and out-patient basis. We shall assess the demographic data, patient's history and management. The aim of these case studies is to have an in-depth idea on the management of rhinosinusitis in Mbingo Baptist Hospital compared to the already addressed international standards.

Statistics of all cases of rhinosinusitis were taken from the ENT department registers from the year 2011 to 2015 (January to September). It is obvious that the prevalence of adult patients with rhinosinusitis is more than two times the prevalence of both the children and teenagers mixed. The total number of children from 2011 to 2015 was 677, teenagers were 514 and adults 3477, for a final total of 4668 people with rhinosinusitis. This may be due to the fact that adult patients have reinfections or relapsed conditions and therefore need to visit the hospital more frequently. This also may show their increase fragility and incapacity to control or to resist the allergens or causative agents. This information is better symbolized on figures 16, 17 and 18. For all of them the number of patients seen with rhinosinusitis was higher in 2014.

From the table indicating the total prevalence of Rhinosinusitis from 2011 to 2015, it is showing that the prevalence of rhinosinusitis is lowest in 2015 with a percentage of 10.5\% and highest in 2011 
(13.9\%). The total average prevalence (from 2011 to 2015) is 11.7\%. Since the department began being functional by 2011, population awareness about this condition was not well spread and resulted to an increase in prevalence in relation to the total number of patients seen in that year, which obviously is the smallest number.

As far as the management of rhinosinusitis is concerned, from the 5 case studies seen, it consisted in general of the following:Normal saline drops, Nasal decongestant, Corticosteroid spray and tablets, Antibiotics, Antihistamines, Functional Endoscopic Sinus Surgery.

To conclude, Rhinosinusitis which is the inflammation of the nasal mucosa, has proved to be a problem in the sub-Saharan African regions as described in the literature review. It is a universal health concern in the sense that it affect anybody and anyway provided the allergens or causative agents are present. This condition can either be acute or chronic, allergic and non-allergic, and can be managed medically and surgical, all depending on the gravity of the condition.

Though the prevalence of rhinosinusitis depends on the realities of each country and even reach up to 35\% in Nigeria, the one identified by this study and representative of the whole country is lower (11.7\%). The fact that this prevalence is around this average value, and considering the type of management done by the health professional which meet standard protocols and practice, we can say that rhinosinusitis is under control. Because population is dynamic, it may difficult to bring that value lower. Proper preventive measures will help reduce the prevalence of rhinosinusitis, among which the intensified education of the population and on preventive measures.

The standard guideline of management of rhinosinusitis will consist of one or the combination of the following:

1. Antihistamine (1 week to 1 month) (may continue for a period of 6 months)

2. Corticosteroids (2 weeks to 1 month)

3. Antibiotics (penicillins or cephalosporins with sometimes with an addition of anaerobes antibiotics like metronidazole) (5 days to 2 weeks dependently)

4. Nasal decongestant (1 to 2 weeks) (if associated with severe nasal congestion)

5. Nasal spray steroids (2 weeks to 1 month) (may continue for a period 6 months)

6. Surgical intervention (Functional Endoscopic Sinus Surgery) for those patients with facial sinuses polyps.

\section{Keywords}

1. AR-Allergic Rhinitis

2. CAR-Chronic allergic rhinitis

3. RS-Rhinosinusitis

4. CRS-Chronic rhinosinusitis

5. MBH- Mbingo Baptist Hospital

6. IgE- immune-globulin $E$

7. IL- interleukin

8. INC-intranasal corticosteroid

9. (F)ESS- (Functional) endoscopic sinus surgery

10.CT-computerized tomography

11.NP- Nasal polyp

12.OMU- ostiomeatal unit

\section{Introduction}

\section{Background and problem statement}

It occurs worldwide and affects about $16 \%$ of the adult American population annually significantly impairing their quality of life. The direct costs associated with rhinosinusitis are higher than the background costs due to increased clinic visits and prescribed medications and the indirect costs due to inactivity days leading to reduced productivity are enormous. It has been found to be one of the 
most costly physical conditions for American citizens with approximately \$6 billion spent overall on its management

Allergic rhinitis (AR) and chronic rhinosinusitis (CRS) are prevalent non communicable diseases increasing around the world, even in Africa. These chronic diseases confer a significant burden through direct or indirect symptoms, complications and cost. In contrast to developed countries, the government health program of many African countries focus only on transmissible diseases, malnutrition, maternal and infant mortality, while data on respiratory diseases remain scarce. The prevalence of AR is very high $(>35 \%)$ among Nigerian Africans, and it is likely that environmental factors are responsible for major differences with other countries. AR prevalence is much higher in urban area, especially in capital cities from Africa. CRS is not less common. The advent of HIVAIDS has further worsened the incidence of CRS in Africa. Local allergens related to African environmental settings are not well known. House dust mites and cockroaches are reported as major allergens in Africa, while pollen allergens remain poorly described. AR predisposes to development of other airway comorbidities such as allergic asthma, rhinosinusitis, nasal polyposis, adenoid hypertrophy and otitis media. Both AR and CRS significantly affect the quality of life of Africans through direct cost (payments to doctors for frequent consultations, prescribed medications, over the counter (OTC) drugs, alternative and complementary drugs), indirect costs (loss of work hours and school days) and intangible costs (loss of quality of life, pain and suffering, psychological maladjustment, social costs). These costs become significant in countries with high prevalence of allergy and low per capital income, as seen both in the Anglophone and Francophone African countries.

Thus the following research question of "what is the prevalence of rhinosinusitis?"

\section{General objective}

Our main objective is to assess the prevalence and management of rhinosinusitis in Mbingo Baptist Hospital.

\section{Specific objective}

More specifically, the investigator will be able:

- $\quad$ To define and describe rhinosinusitis

- $\quad$ To assess the management of rhinosinusitis in MB

- To come out with the prevalence of rhinosinusitis in MBH

- $\quad$ To ascertain the standard management of rhinosinusitis

\section{Hypothesis}

Knowing the prevalence of Rhinosinusitis will serve as reference for many other investigations. This prevalence would tend to increase if the condition is not well managed at the level of the hospital.

\section{Significance of the study}

This study will go a long way to give information on the total number of rhinosinusitis cases from 2011 to 2015, in children, teenagers and adults. It will also go a long way to portray the management of rhinosinusitis with standardised protocols.

\section{Scope of the study}

This study is aimed at assessing the prevalence and management of rhinosinusitis in Mbingo Baptist Hospital, in children, teenagers and adults. 
Texila International Journal of Clinical Research

Volume 3, Issue 2, Dec 2016

\section{Limitations}

Financial and time constraints are the main limitations for this study. Without these the investigator would have worked in all the referral hospitals of the North West Region and why not in the whole Country.

\section{Ethical statement}

This study was approved by Texila American University. Since this was a retrospective study, informed written consent could not be obtained from the study participants. However, records of all participants were anonymised and de-identified for the purpose of such a study.

\section{Conceptual model/ framework}

This model illustrate the pattern through which rhinosinusitis occurs. It all start with a stimulating factors that could be found endogenously or exogenously. Once exposed to the allergens, the process starts by a response to the stimulant and the patient will react by excessive sneezing, nasal blockage, then post nasal drip, headache, facial pains and fever. There may be nasal polyps formation, with halitosis, hoarseness of voice, pharyngitis. 


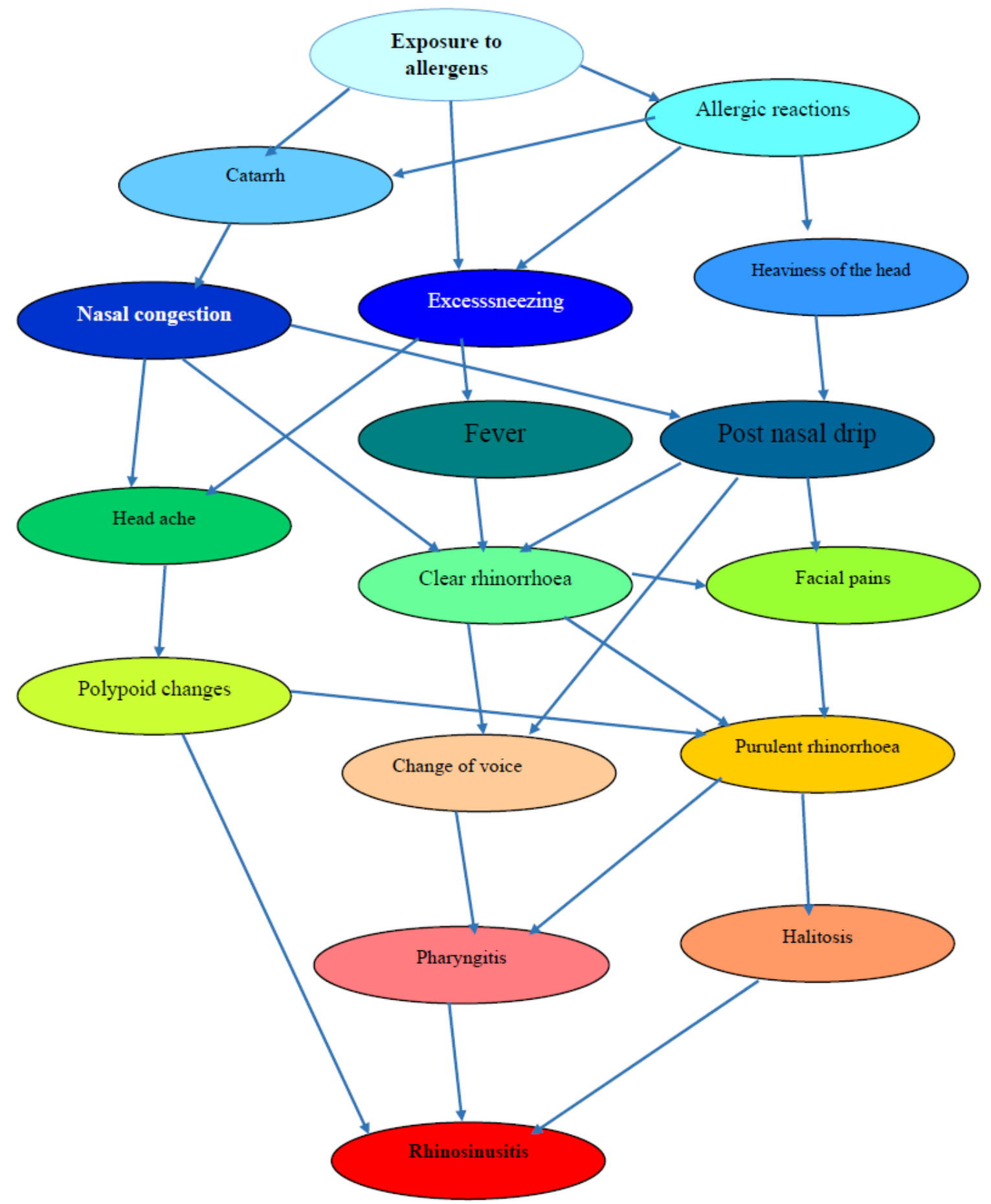

\section{Definition and characteristics}

Rhinosinusitis denotes the concurrent inflammatory and infectious process affecting the nasal cavities and the contiguous paranasal sinuses resulting from the interaction of multiple host and environmental factors. 
Rhinosinusitis, also known as a sinus infection or Sinusitis is inflammation of the sinuses resulting in symptoms. According to the official journal of American Academy of Otolaryngology-Head and Neck Surgery, Chronic sinusitis affects approximately $12.5 \%$ of people.

Sinusitis significantly impacts quality of life measures with decrements in general health perception, vitality and social functioning comparable with that observed in patients who have angina or chronic obstructive pulmonary disease. Sinusitis is one of the main reasons for which an antibiotic is prescribed and for lost productivity in the work force.

Children's sinuses are not fully developed until late in the teen years. Although small, the maxillary (behind the cheek) and ethmoid (between the eyes) sinuses are present at birth. Unlike in adults, paediatric sinusitis is difficult to diagnose because symptoms of sinusitis can be caused by other problems, such as viral illness and allergy.

\section{Classification of rhinosinusitis}

\section{a. By duration of symptoms}

Of the various sub-classifications of rhinosinusitis, the simplest differentiation is based on duration of symptoms. Acute rhinosinusitis is defined by 3 of the guidelines as symptom duration of 4 weeks or less (Clinical Practice Guideline: Adult Sinusitis and the Rhinosinusitis Initiative). The European Position Paper on Rhinosinusitis and Nasal Polyps and British Society for Allergy and Clinical Immunology guidelines qualify acute rhinosinusitis as lasting less than 12 weeks, with complete resolution of symptoms. The Clinical Practice Guideline: Adult Sinusitis includes a category of subacute rhinosinusitis, defined as symptom duration between 4 and 12 weeks, whereas the Joint Task Force on Practice Parameters definition specifies 4 to 8 weeks. Recurrent acute rhinosinusitis is classified by the Clinical Practice Guideline: Adult Sinusitis guidelines as 4 or more episodes of acute rhinosinusitis within 1 year, without persistent symptoms between episodes. The Joint Task Force on Practice Parameters defines recurrent rhinosinusitis as 3 or more episodes per year.

Four of the 5 guidelines (European Position Paper on Rhinosinusitis and Nasal Polyps 2007, British Society for Allergy and Clinical Immunology, Clinical Practice Guideline: Adult Sinusitis, Rhinosinusitis Initiative) designate Chronic rhinosinusitis as symptoms persisting 12 weeks or longer, whereas the Joint Task Force on Practice Parameters indicates 8 weeks.

\section{b. By severity of symptoms}

All 5 guidelines recognize that an assessment of symptom severity is important to define the magnitude of disease and assist with treatment selection. For clinical purposes, the European Position Paper on Rhinosinusitis and Nasal Polyps 2007 and British Society for Allergy and Clinical Immunology guidelines categorize disease severity on the basis of a $10-\mathrm{cm}$ visual analogue scale (VAS) that has been statistically validated for use in patients with RS. Patients responding to the question "How troublesome are your symptoms of rhinosinusitis?" provide a rating, with the scale ranging from 0 ("not troublesome") to 10 ("worst thinkable troublesome").

Scores are categorized as follows, between 0 and 3, mild disease; greater than 3 to 7 , moderate disease; and greater than 7 to 10, severe disease. Scores greater than 5 have been correlated with quality of life detriments.

\section{c. Acute rhinosinusitis}

Acute sinusitis is usually precipitated by an earlier upper respiratory tract infection, generally of viral origin, mostly caused by rhinoviruses, coronaviruses, and influenza viruses, others caused by adenoviruses, human parainfluenza viruses, human respiratory syncytial virus, enteroviruses other than rhinoviruses, and metapneumovirus.

If the infection is of bacterial origin, the most common three causative agents are Streptococcus pneumoniae, Haemophilus influenzae, and Moraxella catarrhalis. Until recently, Haemophilus influenzae was the most common bacterial agent to cause sinus infections. However, introduction of 
the $\mathrm{H}$. influenza type B (HIB) vaccine has dramatically decreased $\mathrm{H}$. influenza type B infections and now non-typable $\mathrm{H}$. influenza (NTHI) are predominantly seen in clinics. Other sinusitis-causing bacterial pathogens include Staphylococcus aureus and other streptococci species, anaerobic bacteria and, less commonly, gram negative bacteria.

Viral sinusitis typically lasts for 7 to 10 days, whereas bacterial sinusitis is more persistent. Approximately $0.5 \%$ to $2 \%$ of viral sinusitis results in subsequent bacterial sinusitis. It is thought that nasal irritation from nose blowing leads to the secondary bacterial infection.

Acute episodes of sinusitis can also result from fungal invasion. These infections are typically seen in patients with diabetes or other immune deficiencies (such as AIDS or transplant patients on immunosuppressive anti-rejection medications) and can be life-threatening. In type I diabetics, ketoacidosis can be associated with sinusitis due to muco-mycosis.

Chemical irritation can also trigger sinusitis, commonly from cigarette smoke and chlorine fumes. Rarely, it may be caused by a tooth infection. (Leung RS, Katial R (March 2008). "The diagnosis and management of acute and chronic sinusitis". Primary care). This will lead to what is called allergic rhinitis.

According to the American Academy of Family Physician, Acute rhinosinusitis has a relatively rapid onset, is normally of four weeks' duration or less and symptoms totally resolve. Most cases are viral in origin. Resolution of symptoms usually occurs within five to seven days, and most patients recover without medical intervention. The subcategory of acute bacterial rhinosinusitis is more likely to evolve into chronic disease or to spread outside the sinuses to the orbital area or to the meninges. Acute bacterial rhinosinusitis is suggested by symptoms including purulent drainage that worsens after five days or persists beyond 10 days, and/or symptoms that are out of proportion to those typically associated with a viral upper respiratory process.6 Recurrent acute rhinosinusitis is defined as four or more episodes of acute disease within a 12-month period, with resolution of symptoms between each episode (each episode of at least seven days' duration). Sub-acute rhinosinusitis is basically a low-grade continuum of acute infection of more than four weeks' but less than 12 weeks' duration. Chronic rhinosinusitis is distinguished by symptoms that persist for 12 weeks or more.

Young children are more prone to infections of the nose, sinus, and ears, especially in the first several years of life. These are most frequently caused by viral infections (colds), and they may be aggravated by allergies. However, if the child remains ill beyond the usual week to ten days, a sinus infection may be the cause.

\section{- Allergic and non-allergic rhinitis}

i. Allergic Rhinitis occurs when the body's immune system over-responds to specific, noninfectious particles such as plant pollens, molds, dust mites, animal hair, industrial chemicals (including tobacco smoke), foods, medicines, and insect venom. During an allergic attack, antibodies, primarily immunoglobin E (IgE), attach to mast cells (cells that release histamine) in the lungs, skin, and mucous membranes. Once IgE connects with the mast cells, a number of chemicals are released. One of the chemicals, histamine, opens the blood vessels and causes skin redness and swollen membranes. When this occurs in the nose, sneezing and congestion are the result.

Seasonal allergic rhinitis or hay fever occurs in late summer or spring. Hypersensitivity to ragweed, not hay, is the primary cause of seasonal allergic rhinitis in 75 percent of all Americans who suffer from this seasonal disorder. People with sensitivity to tree pollen have symptoms in late March or early April; an allergic reaction to mold spores occurs in October and November as a consequence of falling leaves.

Perennial allergic rhinitis occurs year-round and can result from sensitivity to pet hair, mold on wallpaper, houseplants, carpeting, and upholstery. Some studies suggest that air pollution such as automobile engine emissions can aggravate allergic rhinitis. Although bacteria is not the cause of allergic rhinitis, one medical study found a significant number of the bacteria Staphylococcus aureus 
in the nasal passages of patients with year-round allergic rhinitis, concluding that the allergic condition may lead to higher bacterial levels, thereby creating a condition that worsens the allergies.

Patients who suffer from recurring bouts of allergic rhinitis should observe their symptoms on a continuous basis. If facial pain or a greenish-yellow nasal discharge occurs, a qualified ear, nose, and throat specialist can provide appropriate sinusitis treatment.

ii. Non-Allergic Rhinitis does not depend on the presence of IgE and is not due to an allergic reaction. The symptoms can be triggered by cigarette smoke and other pollutants as well as strong odors, alcoholic beverages, and cold. Other causes may include blockages in the nose, a deviated septum, infections, and over-use of medications such as decongestants

\section{d. Chronic rhinosinusitis (CRS)}

Chronic rhinosinusitis (CRS) is a complex disease associated with inflammation of nasal and sinus tissue. Currently, the cause of CRS remains unclear but researchers have proposed several mechanisms. In healthy people, the nose and sinuses are lined with epithelial cells and other specialized cells that form a mucosal barrier. This barrier serves to protect the underlying tissues from the millions of allergens, microbes, and particulates inhaled regularly. Such inhaled particles can be trapped in mucus and cleared through the action of cilia on nasal epithelial cells that move mucus out of the airways. In CRS, the mucosal barrier can be defective. Epithelial cells do not function normally, leading to a weak and permeable barrier. This impaired barrier is exposed to more particles and does not repair itself as well as a healthy barrier. There is increased thick mucus production in CRS that cannot be easily cleared away by nasal epithelial cilia.

Some people with CRS have persistent growth of fungi or bacteria such as Staphylococcus aureus in their nose and sinuses.

In some cases, the organism does not cause significant damage to tissue. However, problems can occur if an immune response is mounted against the colonising organism or to the toxins it makes. This immune response can cause inflammation and damage to the sinus and nasal tissues and worsen CRS.

\section{- Cardinal signs or symptoms}

Prolonged duration of rhinosinusitis (RS) symptoms (more than 8-12 weeks) is the primary reason to evaluate a patient for CRS. In this regard, it is important to distinguish CRS from recurrent ARS, the latter of which is typified by 2 to 4 isolated episodes of ARS per year, with complete resolution of symptoms between episodes. Such episodes should be treated like any other ARS event but also warrant further work-up to investigate potential underlying causes for the recurrence (e.g., AR, cystic fibrosis, immunologic deficiency, ciliary dyskinesia, anatomic abnormalities).

\section{- Factors contributing to sinusitis chronicity}

A similar set of factors contributes to sinusitis chronicity, but in addition other aspects of the host immune microbial interaction play a key role.

\section{- Ostial blockage}

The importance of sinus ostial patency was eloquently stated by Senior and Kennedy: "Sinus health in any patient depends on mucous secretion of normal viscosity, volume, and composition; normal muco-ciliary flow to prevent mucous stasis and subsequent infection; and open sinus ostia to allow adequate drainage and aeration.

While defect of any of these elements can result in acute, recurrent acute or chronic sinusitis, ostial blockage is key in the cycle for the vast majority of sinusitis in asthmatic and nonasthmatic patients alike." The above statement applies to all sinuses, but the sinus ostia most commonly blocked are those that drain through the ostiomeatal unit (OMU). Hence the anterior ethmoid and maxillary sinuses are the most commonly affected sinus areas in both acute and chronic sinusitis. Frontal sinusitis results from obstruction of the nasal frontal duct. Posterior ethmoid and sphenoid sinusitis 
results from obstruction of their respective ostia, which collectively drain through the sphenoethmoidal recess. In chronic sinusitis inflammatory mucosal thickening often persists despite treatment with antibiotics. This further impedes normal mucociliary clearance and may directly obstruct sinus ostia.

\section{- Delayed recovery of mucociliary function}

Mucostasis, hypoxia, microbial products, and chronic inflammation probably all contribute to diminished mucociliary function in chronic sinusitis. Studies are conflicting on whether chronic sinusitis is associated with a significant reduction in ciliary beat frequency, but a decrease in mucociliary clearance has been consistently demonstrated. Other contributing factors to slowing of clearance include changes in the viscoelastic properties of mucus, ciliary loss, and other ultrastructural signs of epithelial damage.

\section{- Mucus “recirculation” and osteitis}

Other factors contributing to sinusitis chronicity include mucus "recirculation" and osteitis. Recirculation of sinus mucus from the maxillary sinus has been described in some patients with an accessory sinus ostium. Secretions exit the sinus through the natural sinus ostium and enter the middle meatus. Some of the secretions then re-enter the maxillary sinus through the accessory ostium, usually located inferior to the OMU (ostiomeatal unit) on the lateral nasal wall. From experience, accessory ostia to the maxillary sinus are quite common (approximately $20 \%$ of cases). Osteitis has been described by histologic analysis of ethmoid bone removed from patients with chronic sinusitis. It may occur as a direct result of infection or as a result of sinus surgery with lack of mucosal preservation. The histologic findings include a marked acceleration in bone turnover with new bone formation, fibrosis, and the presence of inflammatory cells. It has been argued that these changes mimic osteomyelitis in the jaw and that osteitis may therefore represent a form of chronic osteomyelitis and a strong reason for disease recurrence despite surgery or antibiotic use.

\section{- Microbial factors in persistence}

Most studies have pointed to differences between acute and chronic sinusitis in terms of microbial pathogens. In acute sinusitis, the predominant organisms are Streptococcus pneumoniae, Hemophilus influenzae, and (in children) Moraxella catarrhalis.

In studies of chronic sinusitis the most common organisms identified were those described above plus Staphylococcus aureus, coagulase-negative Staphylococcus, and anaerobic bacteria. The relative pathogenicity of the organisms in sinusitis is unknown, with the greatest uncertainty surrounding the role of coagulase-negative Staphylococcus and anaerobes.

Insufficient attention has been given to the potential for emergence of antimicrobial resistance during antibiotic treatment for chronic sinusitis. As demonstrated in the study of Brook et al, Betalactamase-producing bacteria can emerge during antibiotic treatment during the transition from acute to chronic sinusitis. Another possibility is the emergence of intermediate- or high-level penicillin resistance during treatment. This type of resistance, resulting from alterations in penicillin-binding proteins, presently ranges from $28 \%$ to $44 \%$ for S pneumoniae isolates in various regions of the United States. There are very limited data on the prevalence of these isolates in chronic sinusitis, but it appears that isolation of penicillin-resistant pneumococci is most commonly seen in patients with recent use of two or more antibiotics. Many of these organisms also demonstrate multiple drug resistance.

\section{- Inflammatory factors in sinusitis}

Inflammation plays a key role in chronic sinusitis pathogenesis. Infectious and non-infectious stimuli appear to contribute, but the precise role of each in chronic sinusitis remains unclear. Two types of inflammation occur in sinusitis, contributing variably to the clinical expression of disease. Infectious inflammation is most clearly associated with acute sinusitis resulting from either bacterial 
or viral infection. Non-infectious inflammation is so named due to the predominance of eosinophils and mixed mononuclear cells and the relative paucity of neutrophils commonly seen in chronic sinusitis.

\section{Diagnostic measures of RS (Rhinosinusitis)}

Rhinosinusitis (RS) can generally be diagnosed adequately on the basis of clinical findings alone, without the use of special imaging techniques or other assessments. However, the consensus guidelines recognize particular situations in which special assessments may have a role.

Other means include:

- CT- computed tomography. It is good for cases before surgery and for evaluation of cases with recurrent RS. Also, the presence of nasal congestion or blockage, discoloredrhinorrhea or postnasal drip, facial pain or pressure, hyposmia or anosmia together with consistent pathologic findings during nasal endoscopy or on CT scan establishes the diagnosis

- Magnetic resonance imaging (MRI) is overly sensitive to the transient mucosal changes associated with a normal nasal cycle.

- Nasal Endoscopy. Compared with anterior nasal examination, nasal endoscopy provides a better means of examining the middle meatus region and sphenoethmoidal recesses for the presence of purulence associated with RS.

- Nasal Culture. Nasal culture is not generally recommended for the routine work-up of uncomplicated ARS;

- Sinus Puncture. Although rarely indicated for routine patient care, sinus puncture is the methodology considered the criterion standard for confirming bacterial pathogens within the maxillary sinuses.

\section{Management of rhinosinusitis}

i. Topical intranasal corticosteroids (INS) and nasal douching are first-line treatment for RS.

Nasal douching clears the sino-nasal cavity from pathogens and pro-inflammatory mediators. INS targets the inflammatory response underlying nasal congestion, promote osteomeatal complex drainage and occasionally improve the sense of smell. Moreover, a significant reduction in polyp size in cases of CRS with NP (CRSwNP) has been reported. Based on clinical experience, systemic corticoids remain important for the treatment of CRSwNP, as they provide short-term symptom relief. Short term courses of oral corticosteroids are usually given two to three times yearly.

ii. Oral antibiotics together with topical corticosteroids have been proven to act synergistically, though antibiotics are reserved for the acute exacerbations of RS.

Low-dose macrolides (erythromycin, clarithromycin, roxithromycin and azithromycin) are the most studied antibiotics for CRS treatment have been used as a long-term antibiotic treatment in CRS, while topical antibiotics remain an option for refractory cases where traditional topical steroids and oral antibiotics are ineffective.

iii. Nasal irrigation (douching) is a safe, inexpensive method with beneficial effects such as improvement in mucous clearance, enhanced ciliary beat activity, removal of antigens, biofilms or inflammatory mediators.

Intranasal decongestants like xylomethazoline improve sinus ventilation through nasal decongestion, though prolonged use may have an opposite effect resulting in rhinitis medicamentosa.

iv. Antihistamine have a minimal beneficial in CRS patients with concomitant AR.

According to the GALEN study, approximately, 57\% of patients with CRS report symptoms of allergic rhinitis (AR) as well. The correlation with asthma is stronger in those patients with both CRS and AR symptoms.

In the absence of AR, CRS has a positive correlation with a late onset asthma. To sum up, checking for allergy is advocated in CRS patients.

v. Antileukotrienes (montelukast) might have a beneficialeffect in patients with NP (nasal polyp). 
Following the current evolution in phenotyping, more personalized treatments based on the underlying inflammation will most likely be considered in the future.

vi. Surgery

Systematic reviews of cohort studies, large outcomes studies consistently support the safety and efficacy of functional endoscopic sinus surgery (FESS) for chronic rhinosinusitis (CRS) with nasal polyps

\section{Methods}

\section{Description of the study area}

Mbingo Baptist Hospital is the largest of six hospitals run by the Cameroon Baptist Convention Health Services (www.cbchealthservices.org). This referral hospital originally begun as a leprosy settlement in 1952. Currently, it is a 290-bed referral teaching facility providing high quality, affordable and accessible care to those in need, as an expression of Christian love and witness. The hospital is located at 4300 feet elevation in the Northwest Region of Cameroon, $37 \mathrm{~km}$ north of Bamenda, the regional capital. Patients are currently received from throughout Cameroon and some surrounding African countries. The hospital runs a 24 hours service (three shifts system) with an average of 72,000 outpatients seen annually. The hospital is staffed by 15 full time specialty-trained physicians, 20 resident physicians, and a support staff of 600 . It has a security system to safeguard clients, staff, and their

The services provided include Hansen's Disease (Leprosy), general consultations \& Inpatient services, dental services, imaging (X-Ray/Ultrasound), pharmacy, orthopaedic, ENT, Eye, and general surgeries, HIV/AIDS Prevention \& Care Services (PMTCT \& Care and Treatment), a referral laboratory, physiotherapy \& Prosthetic services, technical services, Pan African Academy of Christians Surgeons (PAACS) Program, Christian Internal Medicine Specialization (CIMS), Chaplaincy/Social services, Palliative Care, and Catering Services.

It is in the ENT department that all patients with ENT related issues are being handled. Our study will consist of assessing the prevalence of rhinosinusitis from the ENT department in Mbingo Baptist Hospital.

\section{- The ENT department of mbingo baptist hospital}

The ENT department in Mbingo Baptist Hospital started functioning around the year 2010 by an ENT surgeon, Cameroonian and trained in Kenya. There were a lot of challenges in the beginning as it was a new field and he needed to do everything on his own and then, he gradually "borrowed "some other nurses from various units to assist him in one or two things.

He later on decided to train ENT clinical officers to help in handling most of the clinical activities while he would be busy with surgical activities. With time these clinical officers could also assist him in the theatre activities.

The ENT department is a specialty department. This means that it is dealing with patients having ENT problems.

\section{- Process to reach ENT department and admission process}

When a patient comes to the hospital having ENT issues, the normal process wants that the screener sees the patient first then refers to the OPD doctor when needing special attention. This doctor after his assessment will refer the patient to the ENT department for further assessment and proper management.

When the patient reaches the ENT department, he is being screened and if he needs to be admitted, the admission sheet is filled with the presenting complaints, history, physical examination, impression/diagnosis, orders. After this step, the patient will care and advance some money at the inpatient payment office, then go to the laboratory just near the in-patient payment office for primary lap investigations, then go to the admission room where the auxiliary nurses taking demographic 
information from the patient and his caregiver(s). From here the patient can go to ward to which he is admitted depending on the condition, the age and the gender.

When the patient reaches the ward, he is admitted by nurse and orders are carried and signed and acted upon while the patient is being put in the bed.

\section{Research design, methodology and ethical considerations}

This is a cross sectional study in which data are collected from the hospital registers for patients having rhinosinusitis since 2011 till 2015 (January to September). With these data, we aim at giving the prevalence and management of rhinosinusitis during this period.

The study will also include 5 case studies met in the ENT department during the period of the study both on an in-patient and out-patient basis. We shall assess the demographic data, patient's history and management. The aim of these case studies is to have an in-depth idea on the management of rhinosinusitis in Mbingo Baptist Hospital compared to the already addressed international standards.

\section{Results}

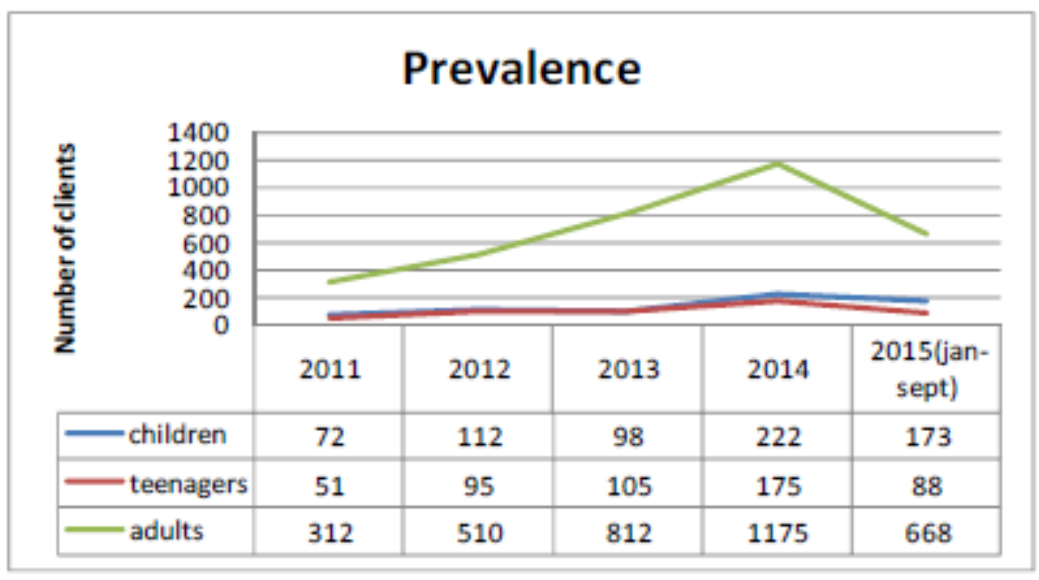

Figure 15. prevalence of rhinosinusitis

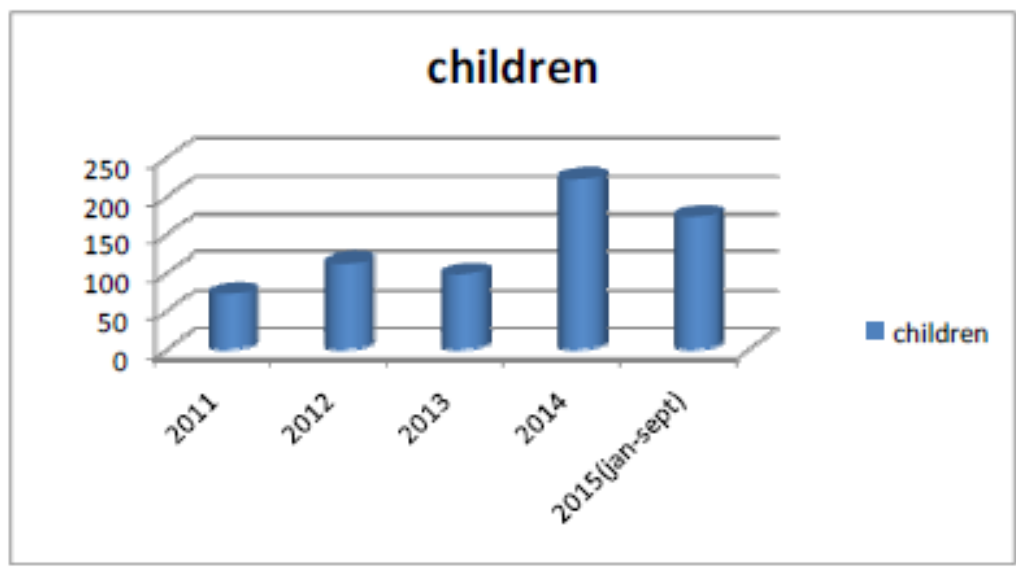

Figure 16. prevalence of children with rhinosinusitis 


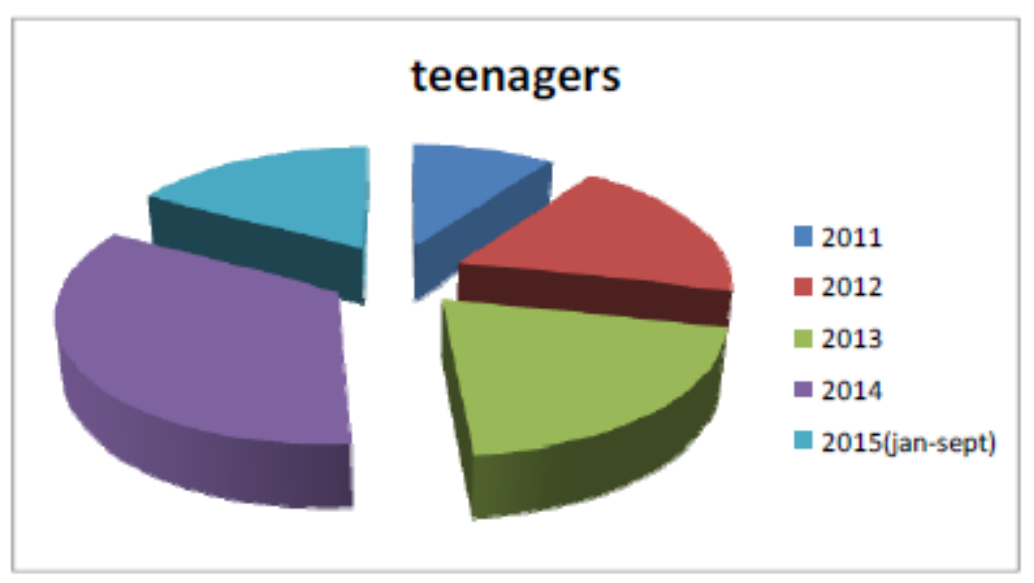

Figure 17. prevalence of rhinosinusitis in teenagers

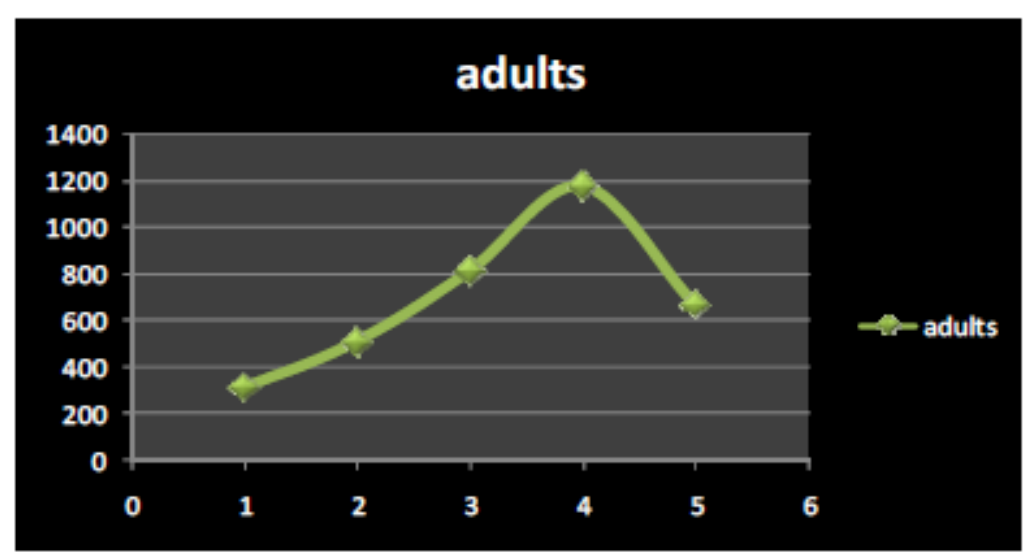

Figure 18. prevalence of rhinosinusitis in adults

Table 1. Total prevalence of Rhinosinusitis from 2011 to 2015

\begin{tabular}{|l|l|l|l|l|l|l|}
\hline Years & 2011 & 2012 & 2013 & 2014 & 2015 & total \\
\hline Total population per year & 3125 & 6682 & 9244 & 11803 & 8850 & $\mathbf{3 9 7 0 4}$ \\
\hline $\begin{array}{l}\text { Total number of patients } \\
\text { with rhinosinusitis }\end{array}$ & 435 & 714 & 1015 & 1572 & 929 & $\mathbf{4 6 6 5}$ \\
\hline Prevalence per year (\%) & $13.9 \%$ & $10.7 \%$ & $11 \%$ & $13.3 \%$ & $10.5 \%$ & $\begin{array}{l}\mathbf{5 9 . 4 \%} \\
\text { Average= } \\
\mathbf{1 1 . 7} \%\end{array}$ \\
\hline
\end{tabular}




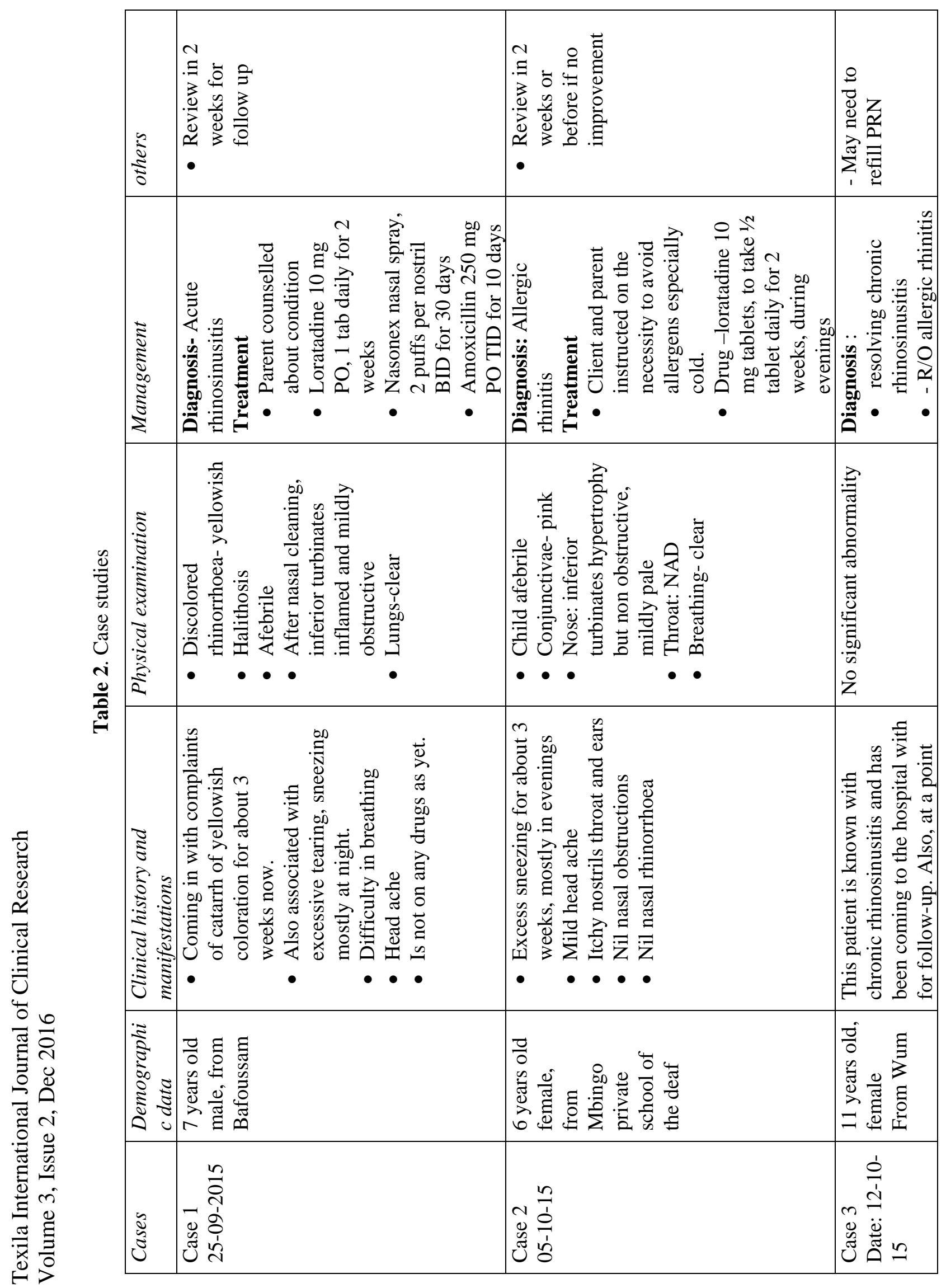




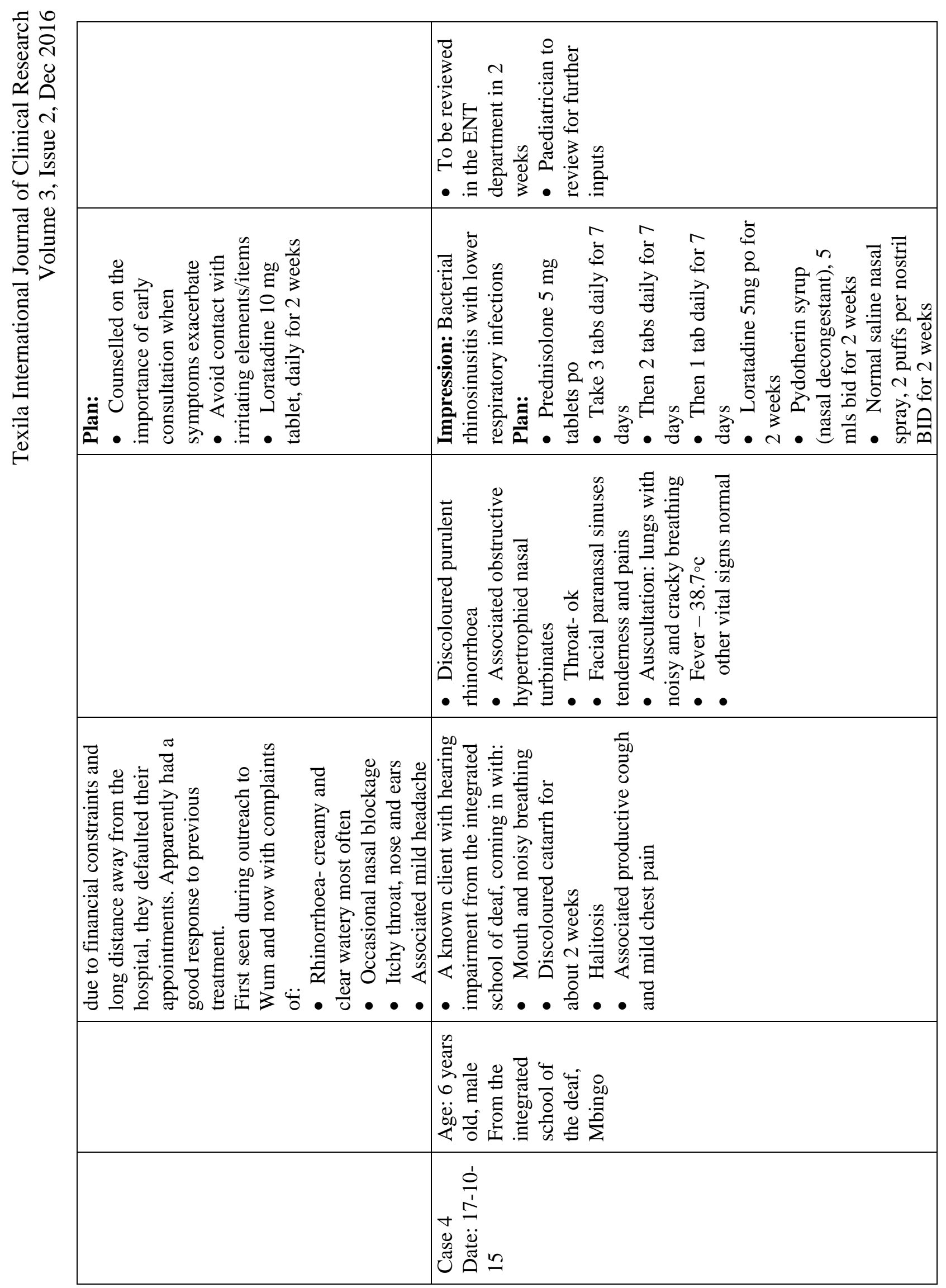




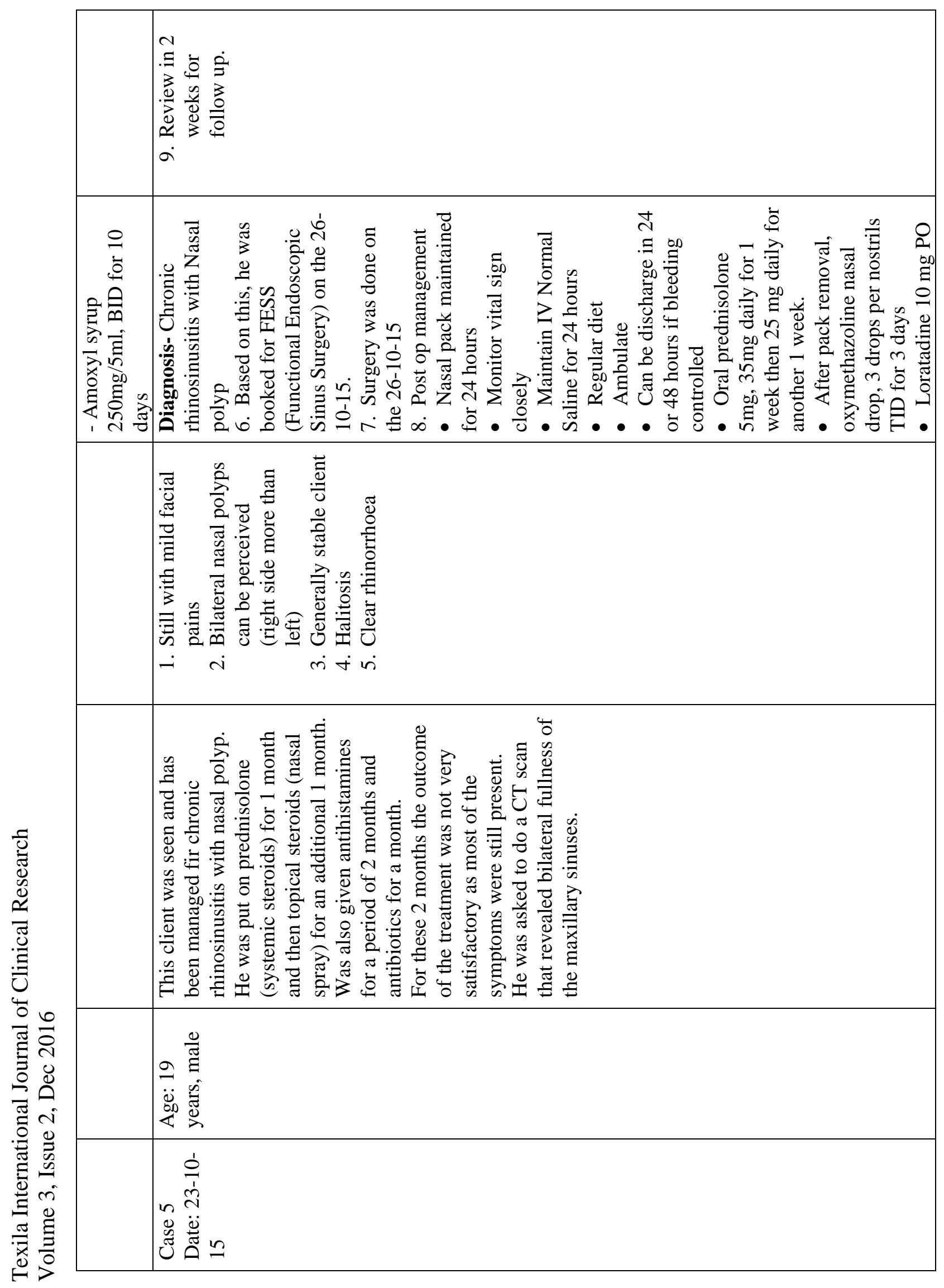




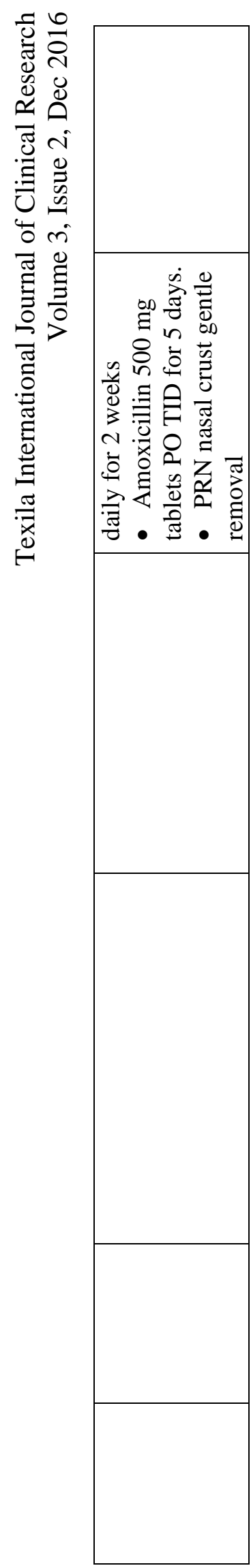




\section{Discussion of results}

\section{Prevalence of rhinosinusitis}

Statistics of all cases of rhinosinusitis were taken from the ENT department registers from the year 2011 to 2015 (January to September). It is obvious that the prevalence of adult patients with rhinosinusitis is more than two times the prevalence of both the children and teenagers mixed. The total number of children from 2011 to 2015 was 677; teenagers were 514 and adults 3477, for a final total of 4668 people with rhinosinusitis. This may be due to the fact that adult patients have reinfections or relapsed conditions and therefore need to visit the hospital more frequently. This also may show their increase fragility and incapacity to control or to resist the allergens or causative agents. This information is better symbolized on figures 16, 17 and 18. For all of them the number of patients seen with rhinosinusitis was higher in 2014.

From the table indicating the total prevalence of Rhinosinusitis from 2011 to 2015, it is showing that the prevalence of rhinosinusitis is lowest in 2015 with a percentage of $10.5 \%$ and highest in 2011 (13.9\%). The total average prevalence (from 2011 to 2015) is $11.7 \%$. Since the department began being functional by 2011, population awareness about this condition was not well spread and resulted to an increase in prevalence in relation to the total number of patients seen in that year, which obviously is the smallest number.

\section{Management of rhinosinusitis}

As far as the management of rhinosinusitis is concerned, from the 5 case studies seen, the first case, a 7 years old male, with a diagnosis of acute rhinosinusitis, was managed with antihistamine, a steroid nasal spray, antibiotic and counselled about condition.

The second case, was a 6 years old female, diagnosed with allergic rhinitis (acute). She was managed with an anti-histamine only

The third case, 11 years old female, was first seen during a community outreach and was treated. She was referred to the hospital for follow up. So she had resolving chronic rhinosinusitis with associated allergies. She was given only an antihistamine and was seriously counselled on the importance of early consultation with relapse.

The fourth case, 6 years old boy, was one of the complicated type. He was diagnosed with bacterial rhinosinusitis with associated lower respiratory tract infections. He was prescribed corticosteroids tablets, nasal decongestant, anti-histamines, and antibiotics. He was later referred to the paediatrician for more inputs.

The fifth but not the least case, was a 19years old male. This case too has been followed up by the ENT department and was diagnosed to have chronic rhinosinusitis with nasal polyps. He was first place on corticosteroid (topical spray and tablets) and antibiotics for some months of treatment. He had persistent symptoms and was asked to do a CT scan which showed bilateral maxillary sinuses fullness with Polypoid changes. He later had a Functional Endoscopic Sinus Surgery. After this he was managed as follows on discharge home:

1. Nasal douching solution (normal saline drops)

2. Nasal decongestant

3. Corticosteroid spray and tablets

4. Antibiotics

5. Antihistamines

\section{Conclusion}

Rhinosinusitis which is the inflammation of the nasal mucosa, has proved to be a problem in the sub-Saharan African regions as described in the literature review. It is a universal health concern in the sense that it affect anybody and anyway provided the allergens or causative agents are present. 
This condition can either be acute or chronic, allergic and non-allergic, and can be managed medically and surgical, all depending on the gravity of the condition.

Though the prevalence of rhinosinusitis depends on the realities of each country and even reaches up to $35 \%$ in Nigeria, the one identified by this study and representative of the whole country is lower $(11.7 \%)$. The fact that this prevalence is around this average value, and considering the type of management done by the health professional which meet standard protocols and practice, we can say that rhinosinusitis is under control. Because population is dynamic, it may difficult to bring that value lower. Proper preventive measures will help reduce the prevalence of rhinosinusitis, among which the intensified education of the population and on preventive measures.

The standard guideline of management of rhinosinusitis will consist of one or the combination of the following:

1. Antihistamine (1 week to 1 month) (may continue for a period of 6 months)

2. Corticosteroids (2 weeks to 1 month)

3. Antibiotics (penicillins or cephalosporins with sometimes with an addition of anaerobes antibiotics like metronidazole) (5 days to 2 weeks dependently)

4. Nasal decongestant (1 to 2 weeks) (if associated with severe nasal congestion)

5. Nasal spray steroids (2 weeks to 1 month) (may continue for a period 6 months)

6. Surgical intervention (Functional Endoscopic Sinus Surgery) for those patients with facial sinuses polyps.

\section{Recommendations}

- Mbingo Baptist Hospital should include allergic test in the lab investigations. In Mbingo, they base themselves mostly on clinical histories and complaints from the patients. This test will help differentiate between allergic rhinitis and non-allergic rhinitis.

- Increased education of the population on the condition (rhinosinusitis), its causative agents, its complications, and preventive measures would go a long way to reduce its prevalence.

- There is a need to have a CT scan in the Hospital. All patients that need CT scan are referred to some other places (like in Bafoussam, Douala or Yaoundé) and sometimes out of Bamenda town, to have the CT done, when the scan in Bamenda town is broken.

- Further studies may demonstrate the reasons behind this increase in the prevalence of rhinosinusitis in adults more than in children and teenagers.

\section{References}

[1]. Bhattacharyya N. Contemporary assessment of the disease burden of sinusitis. Am J Rhinol Allergy. 2009;23(4):392-395

[2]. Brook I, Frazier EH, Foote PA. Microbiology of chronic maxillary sinusitis: comparison between specimens obtained by sinus endoscopy and by surgical drainage. J Med Microbiol 1997; 46:430-2.

[3]. Brook I, Yocum P, Frazier EH. Bacteriology and beta-lactamase activity in acute and chronic maxillary sinusitis. Arch Otolaryngol Head Neck Surg 1996; 122:418-23.

[4]. Brook I. Bacteriologic features of chronic sinusitis in children. JAMA 1981; 246:967-9.

[5]. Fokkens W, Lund V, Mullol J, European Position Paper on Rhinosinusitis and Nasal Polyps Group European position paper on rhinosinusitis and nasal polyps 2007. Rhinology. 2007;45 (suppl 20):1-139 http://www.ep3os.org/EPOS2007.pdf Accessed February 16, 2011

[6]. Fokkens WJ, Lund VJ, Mullol J, Bachert C, Alobid I, Baroody F, et al. EPOS 2012: European position paper of rhinosinusitis and nasal polyps 2012. Rhinol Suppl 2012:1- 298.

[7]. Gliklich RE, Metson R. The health impact of chronic sinusitis in patients seeking otolaryngologic care. Otolaryngol Head Neck Surg. 1995;113(1):104-109 [PubMed]

[8]. Global atlas of allergic rhinitis and chronic rhinosinusitis. 2015

[9]. Jarvis D, Newson R, Lotvall J, Hastan D, Tomassen P, Keil T, et al. Asthma in adults and its association with chronic rhinosinusitis: the GA2LEN survey in Europe. Allergy 2012; 67:91-98. 
Texila International Journal of Clinical Research

Volume 3, Issue 2, Dec 2016

[10]. Klossek JM, Dubreuil L, Richet H, Richet B, Beutter P. Bacteriology of chronic purulent secretions in chronic rhinosinusitis. J LaryngolOtol 1998; 112:1162-6.

[11]. Pleis JR, Lucas JW, Ward BW. Summary health statistics for U.S. adults: National Health Interview Survey, 2008. National Center for Health Statistics. Vital Health Stat 10. 2009; (242):1-157 www.cdc.gov/nchs/data/series/sr_10/sr10_242.pdf Accessed February 16, 2011 [PubMed]

[12]. Prokopakis E, Vlastos I, Pant H, Ferguson BJ. Chronic rhinosinusitis observation or treatment. CurrOpin Allergy ClinImmunol 2013; 13:31-36.

[13]. Prokopakis EP, Hellings PW, Velegrakis GA, Kawauchi H. From ancient Greek medicine to EP3OS. Rhinology 2010; 48:265-272.

[14]. Prokopakis EP, Vlastos IM, Ferguson BJ, Scadding G, Kawauchi H, Georgalas C, et al. SCUAD and chronic rhinosinusitis. Reinforcing hypothesis driven research in difficult cases. Rhinology 2014; 52:3-8.

[15]. Ramadan HH. What is the bacteriology of chronic sinusitis in adults? Am J Otolaryngol 1995; 16:303-6.

[16]. Rontal M, Bernstein JM, Rontal E, Anon J. Bacteriologic findings from the nose, ethmoid, and bloodstream during endoscopic surgery for chronic rhinosinusitis: implications for antibiotic therapy. Am J Rhinol 1999; 13:91-6. 


\title{
Break the Germ Cycle: Hand Hygiene Compliance among Healthcare Workers in an Accredited Tertiary Care Hospital
}

\author{
Article By Jessy Sebastian \\ MSN, Texila American University, United Arab Emirates \\ Email: Jessysebastian4@Yahoo.Com
}

\begin{abstract}
Health care associated infections affect hundreds of millions of people around the world and it is a major global issue for patient safety. Moreover, the most common way that micro-organisms (germs), particularly bacteria, are spread and cause infection is by being carried on people's hands. Hand hygiene is the most important measure to avoid the spread of harmful germs and to prevent ill health. Regular and thorough hand hygiene is always important when working in an environment or organization where health care is provided. So, having clean hands helps to protect patients, particularly the most vulnerable, as well as health care workers.

This change project proposed to improve hand hygiene compliance among health care workers in Intensive care units (Cardiac, Medical \& Neonatal ICU's) of a tertiary hospital in UAE. This included increasing compliance to hand washing, as well as introducing another simple, quick and effective procedure of hand hygiene which is using alcohol hand rub. The hand hygiene compliance rate showed gradual improvement over time, starting from below standard hand hygiene at base line assessment (44\%), to reach its maximum at 3 weeks post intervention assessment (94\%). These results indicate that the systematic application of the change model and WHO multimodal strategies is associated with improvement in hand hygiene compliance of healthcare workers and thus it could result in a sustained positive impact.

Although hand washing was found to be the preferred method for hand hygiene at 3 weeks into the intervention, yet the preference was reversed in the 3 weeks post intervention assessment to alcohol hand rub. In general, the literature suggests healthcare workers are possibly more concerned about their own safety than transmitting infection to patient. Yet in this project, they are found to be in different to the two indications with similar levels of compliance at baseline and postintervention.
\end{abstract}

\section{Introduction}

\section{Background of study}

Hand hygiene (i.e., washing hands with soap and water, or disinfection using alcohol- based hand rub) remains the single most important measure of preventing the spread of antimicrobial resistant pathogens and subsequent nosocomial infection. (Barrs A, 2000 and Pittet. et al 2006). Hand washing or hand hygiene is an ancient cultural custom that goes back to an immemorial time. It was observed primarily to remove dirt and to relieve people symbolically from physical and moral evils.

Barbacombe (2004) suggests -it is so basic, so simple, almost insulting or embarrassing even to mention, especially at an advanced practice level. Unfortunately, in health care, compliance with hand hygiene practices has been below an acceptable level. One study aimed to measure medical staff attitude towards hand hygiene; this showed compliance rates of hand cleaning of less than $50 \%$ (Bischoff, Reynolds, Scssler, Edmond and Wenzel, 2000). Another study done on a similar topic showed a compliance rate of $63 \%$. (Suchitra \& Lakshmidevi, 2006). These studies demonstrate that hand hygiene practices are below an acceptable level. So failure to perform appropriate hand hygiene either by washing or disinfection by any means or technique is considered to be the leading cause of infection. 
Every day, consciously and unconsciously, we make decisions regarding our patients' care. To make clinical decisions, almost instinctively we resort to variety of resources, including our own clinical experiences and discussions with colleagues - we rely on textbooks, journal articles, and previous educational experiences. As educators, we should evaluate the methodology of teaching students and residents how to make clinical decisions. Evidence-based practice (EBP) is an approach to health care wherein health professionals use the best evidence possible, i.e. The most appropriate information available, to make clinical decisions for a patient. EBP values, enhances, and builds on clinical expertise, knowledge of disease mechanisms, and patho-physiology (Macnee, 2004).

Over the past few years, scientific evidence to support the role of hand hygiene in the improvement of patient safety has increased considerably, but some key controversial issues still challenge care practitioners and researchers. Key among these is the question of compliance in practice and it is now recognized that there is no single solution; rather multimodal programmes are needed to promote compliance (Pittet D et al, 2000).

\section{Objective of the study}

-To evaluate compliance with the opportunity of hand hygiene by health care personnel within 3 Intensive Care Units in my Hospital, following the implementation of WHO Hand Hygiene Implementation multimodal improvement strategy and applying the HSE change model

- To understand the behavioral determinants of hand hygiene in the 3 Intensive Care Units

- To assess the health care workers (HCW) knowledge \& and perception/attitude change in response to the implementation multimodal improvement strategy namely the hand hygiene training program

\section{Significance of the study}

Health care associated infections affect hundreds of millions of people around the world and it is a major global issue for patient safety (WHO, 2009). In general, infections have a multifactorial causation related to systems and processes of health care provision as well as to political and economic constraints on health systems (WHO, 2009). Moreover, the most common way that microorganisms (germs), particularly bacteria, are spread and cause infection is by being carried on people's hands (CDC, 2002).

Hand hygiene is the most important measure to avoid the spread of harmful germs and to prevent ill health (Pittet, Allegranzi \& Storr, 2008). Regular and thorough hand hygiene is always important when working in an environment or organisation where health care is provided. So, having clean hands helps to protect patients, particularly the most vulnerable, as well as protecting health care workers (WHO, 2005).

\section{Research Questions}

1. Does implementation of WHO Hand Hygiene Implementation Multi-modal improvement strategy increasethe compliance of Hand Hygiene?

2. Will applying the HSE change modeldecrease Health care associated infections?

\section{Scope of study}

This quality improvement project aimed to remove the Hawthorne effect and improve the behavior of Healthcare workers in Intensive Care Units in a tertiary Hospital.

\section{Limitation}

The limitations of this project were based on the health care workers performing Hand hygiene. The study mainly focussed on the assessment of the behaviour of the health care workers towards hand hygiene. 


\section{Literature Review}

\section{Introduction}

There is a wealth of literature reviewed in this chapter that supports the importance of hand hygiene to preventing healthcare associated infections (HCAIs). The impact of these nosocomial infections is both a direct risk to patients and also increases the service and financial burden to health systems. As a significant safety hazard, multimodal strategies for hand hygiene have been developed as an evidence based global response. In addition, this chapter considers hand hygiene from the historical and cultural perspectives, as well as the main factors affecting compliance.

\section{Method of literature review}

Searches of the literature were conducted using RCSI library resources. I mainly used Science direct and Medline. Other resources include Google scholar, but most of the useful research articles found were not accessible. Also I was lucky enough to have the password for HINARI which is a good web link offered by the World Health Organization that gives access to most of the international journals.

The search terms used were: - healthcare associated infection - hand hygiene compliance - hand hygiene and compliance - hospital acquired infection - hand washing compliance - hand washing and compliance - hand disinfection compliance - hand rub compliance-hand decontamination compliance.

The related articles for each search were reviewed according its relevance. The criteria of selection included: the original research article or a systematic review article, studies exploring compliance of hand hygiene among health care workers and the language in English. Relevant papers were selected by screening the title (first step), the abstracts (second step), then the entire articles (third step) retrieved during the database searches.

\section{Healthcare-Associated Infections:}

Healthcare-Associated Infections (HCAIs) represent a major risk to patient safety and contribute towards suffering, prolongation of hospital stay, cost and mortality (Cosgrove, 2006 \& Graves, Weinhold and Tong E, et al 2007). In addition to that, the impact of HCAIs implies long-term disability, increases resistance of microorganisms to antimicrobials, adds massive additional financial burdens, increases fatalities, poses a high costs for the health systems, let alone the emotional stress for patients and their families.

Although the risk of acquiring HCAI is universal and pervades every health-care facility and system around the world, the global burden is unknown because of the difficulty of gathering reliable diagnostic data (WHO, 2009). Overall estimates indicate that more than 1.4 million patients worldwide in developed and developing countries are affected at any time. (World Alliance for Patient Safety, 2005).

In developed countries, HCAI concerns $5-15 \%$ of hospitalized patients and can affect $9-37 \%$ of those admitted to intensive care units. (WHO, 2005 \& Vincent, 2003). While in developing countries, many additional factors contribute to increase the risk of HCAI, namely: poor hygiene and sanitation, lack or shortage of basic equipment, and inadequate structures and overcrowding, an unfavorable social background and a population largely affected by malnutrition and other types of infection and/or diseases (Ofner-Agostini et al., 2006 ) \& Ho PL, Tang XP, Seto WH., 2003).

Limited data on HCAI from these developing country settings are available from the literature (WHO, 2009). In addition to the usual difficulties to define the diagnosis of HCAI, there is also paucity and unreliability of laboratory data, lack of standardized information from medical records, and scarce access to radiological facilities. The costs of managing HCAI are likely to represent a higher percentage of the health or hospital budget in low income countries as well.

Healthcare workers (HCWs) can also become infected during patient care as the risk of transmission is two-way. During the Marburg viral hemorrhagic fever event in Angola, transmission 
within health care settings played a major role on the amplification of the outbreak (WHO, 2009). Nosocomial clustering, with transmission to HCWs, was a prominent feature of severe acute respiratory syndrome (SARS). (Ofner-Agostini et al., 2006 )\& Ho PL, Tang XP, Seto WH.(2003). Similarly, HCWs were infected during the influenza pandemics (Jensen et al, 2005). In some settings (Brazil and Indonesia), more than half the neonates admitted to neonatal units acquired a HCAI, with reported fatality rates between $12 \%$ and 52\%( Allegranzi et al (2008).

\section{Transmission of health care-associated infections}

Transmission of health care-associated pathogens takes place through direct and indirect contact, droplets, air and a common vehicle. Transmission occurs mostly via large droplets, direct contact with infectious material or through contact with inanimate objects contaminated by infectious material. Performance of high-risk patient care procedures and inadequate infection control practices contribute to the risk. Transmission of other viral (e.g. human immunodeficiency virus (HIV), hepatitis B) and bacterial illnesses including tuberculosis to HCWs is also well known. (Jensen et al, 2005). Transmission of health care-associated pathogens from one patient to another via HCWs' hands requires five sequential steps (Girou and Oppein, 2001) these are:

(i) Organisms are present on the patient's skin, or have been shed onto inanimate objects immediately surrounding the patient; Health care associated pathogens can be recovered not only from infected or draining wounds, but also from frequently colonized areas of normal, intact patient skin. Lowbury, 1969) \& Bhalla, Aron and Donskey, 2007 ).

(ii) Organisms must be transferred to the hands of HCWs;

(iii) Organisms must be capable of surviving for at least several minutes on HCWs' hands; Several studies have shown the ability of microorganisms to survive on hands for differing times (Musa \& Desai, 1990).

These studies clearly demonstrate that contaminated hands could be vehicles for the spread of certain viruses and bacteria. Whether care is provided to adults or neonates, both the duration and the type of patient care affect HCWs' hand contamination. HCWs' hands become progressively colonized with commensal flora as well as with potential pathogens during patient care. (Pittet et al., 1999, \& Pessoa-Silva et al, 1999). Bacterial contamination increases linearly over time. Pittet et al, 1999). In the absence of hand hygiene action, the longer the duration of care, the higher the degree of hand contamination. The dynamics of hand contamination are similar on gloved versus ungloved hands; gloves reduce hand contamination, but do not fully protect from acquisition of bacteria during patient care. Therefore, the glove surface is contaminated, making cross-transmission through contaminated gloved hands likely.

(iv) For transmission to occur, hand washing or hand antisepsis by the HCW must be inadequate or entirely omitted, or the agent used for hand hygiene inappropriate. When HCWs fail to clean their hands between patient contact or during the sequence of patient care - in particular when hands move from a microbiologically contaminated body site to a cleaner site in the same patient - microbial transfer is likely to occur (Gupta A et al, 2004). To avoid prolonged hand contamination, it is not only important to perform hand hygiene when indicated, but also to use the appropriate technique and an adequate quantity of the product to cover all skin surfaces for the recommended length of time.

(v) The contaminated hand or hands of the caregiver must come into direct contact with another patient or with an inanimate object that will come into direct contact with the patient. Crosstransmission of organisms occurs through contaminated hands. Factors that influence the transfer of microorganisms from surface to surface and affect cross-contamination rates are the type of organism, source and destination surfaces, moisture level, and size of inoculums (Harrison, 2003) showed that contaminated hands could contaminate a clean paper towel dispenser and vice versa. The transfer rates ranged from $0.01 \%$ to $0.64 \%$ and $12.4 \%$ to $13.1 \%$, respectively. 


\section{Health care associated infection and hand hygiene}

It is an apparent testimonial to its importance that health care associated infection is a significant patient safety hazard and continues to harm patients in the 21st century. Much of this harm can be avoided through better application of measures which already exist, including universal implementation of hand hygiene improvement methods.

There is substantial evidence that hand antisepsis reduces the transmission of health care-associated pathogens and the incidence of HCAI (Boyce and (Pittet, 2002), (Larson, 1988) \& Larson, 1999). Semmelweis demonstrated in 1847 that the mortality rate among mothers delivering at the First Obstetrics Clinic at the General Hospital of Vienna was significantly lower when hospital staff cleaned their hands with an antiseptic agent than when they washed their hands with plain soap and water (Larson, 1988). In the 1960s, a prospective controlled trial compared the impact of no hand washing versus antiseptic hand washing on the acquisition of $S$. Aureus among infants in a hospital nursery (Mortimer, 1962) The investigators demonstrated that infants cared for by nurses who did not wash their hands after handling an index infant colonized with $S$. aureus acquired the organism significantly more often, and more rapidly, than did infants cared for by nurses who used hexachlorophene to clean their hands between infant contacts. This trial provided compelling evidence that compared with no hand washing, hand cleansing with an antiseptic agent between patient contacts reduces transmission of health care associated pathogens.

In addition to these studies, outbreak investigations have suggested an association between infection and understaffing or overcrowding that was consistently linked with poor adherence to hand hygiene (Fridkin et al, 1996) These findings show indirectly that an imbalance between workload and staffing leads to relaxed attention to basic control measures, such as hand hygiene, and spread of microorganisms. Hand hygiene is therefore a core element to protect patients against HCAIs and colonization with multiresistant micro-organisms (Pittet et al, 2006).

\section{Global response}

The World Alliance for Patient Safety is an evolving program of the WHO which was established to raise the profile of patient safety within the global health care agenda. The decision taken in 2004 was to focus the effort and attention of the First Global Patient Safety Challenge on the problem of health care-associated infection. Action on hand hygiene improvement is considered at the core of the First Challenge, with field testing of the WHO implementation strategies developed in conjunction with the WHO Guidelines on Hand Hygiene in Health Care.(WHO, 2008).

The First Global Patient Safety Challenge of the WHO World Alliance for Patient Safety, entitled - Clean Care is Safer Cart has followed a classic approach to health improvement. It called for a concerted global effort to effect policy and intervention strategies to enhance patient safety through implementation of a simple, low-cost health improvement intervention (improved compliance with hand hygiene in health care) to contribute to the prevention of HCAI. To achieve its aims, it has required an action plan for each country, and has involved lobbying for national political action on hand hygiene improvement.

This action positions hand hygiene improvement in health care as a key component of an infection control/quality and safety health improvement program (Runciman \& Moller, 2008). Hand hygiene is considered to be the primary measure necessary for reducing HCAI. Although the action of hand hygiene is simple, the lack of compliance among health-care workers continues to be a problem throughout the world (WHO, 2009)

Principally, the Challenge was conceived to facilitate global awareness-raising about the issue of HCAI and its connection with hygiene and cleanliness in health care settings, focusing attention on the burden and impact of these infections on infection control and patient safety. The implementation of the challenge is built upon four predetermined key success factors. First is to raise worldwide awareness of the impact of healthcare-associated infections on patient safety and promote an exchange of information; second is to build commitment from countries to give priority to reducing 
healthcare-associated infections; third is to issue recommendations bringing the scientific evidence for optimal promotion of hand hygiene and to identify a strategy for their implementation; then lastly is to test the implementation of the hand hygiene recommendations in specific sites worldwide as part of an integrated package of actions in the areas of clean products (blood safety), clean practices (safe clinical procedures), clean equipment (injection and immunization safety), and clean environment (safe water and sanitation in healthcare) (Allegranzia et al, 2007).

\section{Hand Hygiene: Definitions and historical perspective}

The word hygiene is derived from the name Hygeia, who was the Greek goddess of healing (Encyclopedia). In modern usage, hygiene usually refers to cleanliness and especially to any practice which leads to the absence or reduction of harmful infectious agents. The term hand hygiene includes hand washing (washing hands with non-antimicrobial soap), antiseptic hand wash (washing hands with water and soap or another detergent containing an antiseptic agent), antiseptic hand rub (rubbing hands with an antiseptic hand rub) and surgical hand antisepsis (preoperative antiseptic hand wash or Hand rub performed by surgical personnel). (Centres for Disease Control and Prevention, 2002).

Although hand washing has been considered a measure of personal hygiene for centuries, the specific link between hand washing and the spread of infectious diseases has been reported only during the last 200 years (WHO, 2009). In the mid-1800s, studies by Ignaz Semmelweis in Vienna, Austria, and Oliver Wendell Holmes in Boston, USA, established that hospital-acquired diseases were transmitted via the hands of HCWs. Semmelweis recommended that hands be scrubbed in a chlorinated lime solution before every patient contact and particularly after leaving the autopsy room.

The 1980s represented a landmark in the evolution of concepts of hand hygiene in health care. The first national hand hygiene guidelines were published in the 1980s, (Simmons, 1981, Garner et al, 1986 and Bjerke, 2004) followed by several others in more recent years in different countries. In 1995 and 1996, the CDC/Healthcare Infection Control Practices Advisory Committee (HICPAC) in the USA recommended that either antimicrobial soap or a waterless antiseptic agent be used for cleansing hands upon leaving the rooms of patients with multidrug-resistant pathogens ((HICPAC, 1995 and Garner, 1996).

The present WHO guidelines are based on the previous document and represent the most extensive review of the evidence base related to hand hygiene. They guidelines aim to expand the scope of recommendations to a global perspective, foster discussion and expert consultation on controversial issues related to hand hygiene in health care, and to propose a practical approach for successful implementation (WHO, 2009).

\section{Religious and cultural aspects of hand hygiene}

Personal hygiene is a key component of human well-being regardless of religion, culture or place of origin. Human health related behaviour, however, results from the influence of multiple factors affected by the environment, education, and culture. So there are several reasons why religious and cultural issues should be considered when dealing with hand hygiene and planning a strategy to promote it in health-care settings. In the increasingly multicultural, globalized community that is health-care provision today, cultural awareness has never been more crucial for implementing good clinical practice in keeping with scientific developments. Immigration and travel are more common and extensive than ever before as a result of the geopolitically active forces of migration, asylumseeking and, in Europe, the existence of a broad, borderless multistate

Union (WHO, 2009). With the increasingly diverse populations accompanying these changes, very diverse cultural beliefs are also more prevalent than ever. This evolving cultural topography demands new, rapidly acquired knowledge and highly sensitive, informed insights of these differences, not only among patients but also among HCWs who are subject to the same global forces.

It is clear that cultural - and to some extent, religious - factors strongly influence attitudes to inherent community hand washing which, according to behavioural theories are likely to have an impact on compliance with hand cleansing during health care (WHO, 2009). 
Hand hygiene can be practised for hygienic reasons, ritual reasons during religious ceremonies, and symbolic reasons in specific everyday life situations. Islam for example, have precise rules for hand washing included in the holy texts and this practice punctuates several crucial moments of the day (WHO, 2009).

Of the five basic tenets of Islam, observing regular prayer five times daily is one of the most important pillars. Personal cleanliness is paramount to worship in Islam ( Lawrence \& Rozmus, 2001). Muslims must perform methodical ablutions before praying, and clear instructions are given in the Qur'an as to precisely how these should be carried out (Muftic, 1997). The Prophet Mohammed always urged Muslims to wash hands frequently and especially after some clearly defined tasks (Katme, 1999).

Ablutions must be made in freely running (not stagnant) water and involve washing the hands, face, forearms, ears, nose, mouth and feet, three times each. Additionally, hair must be dampened with water. Thus, every observant Muslim is required to maintain scrupulous personal hygiene at five intervals throughout the day, aside from his/her usual routine of bathing as specified in the Qur'an. These habits transcend Muslims of all races, cultures and ages, emphasizing the importance ascribed to correct ablutions. (Ahmed et al, 2006).

In contrast, the Christian faith only specifically requires the ritual sprinkling of holy water on hands before the consecration of bread and wine, and washing of hands after touching the holy oil (the latter only in the Catholic Church). In general, the indications given by Christ's example refer more to spiritual behaviour, but the emphasis on this specific point of view does not imply that personal hygiene and body care are not important in the Christian way of life (Whitby, McLaws \& Ross, 2006).

\section{Variation in Hand Hygiene Compliance}

Multimodal programmes for increasing hand hygiene compliance are now recommended as the most reliable, evidence-based method for ensuring sustainable improvement. (Pittet D et al, 2000). WHO has developed and tested a multimodal Hand Hygiene Improvement Strategy to translate into practice the present guidelines. Unfortunately, compliance with hand hygiene practices has been below an acceptable level or very low in both developed and developing countries. (WHO, 2006). So, improvement of compliance with hand hygiene is needed.

Reasons which explain insufficient compliance or suboptimal ractices are multiple and may vary according to the health care setting and the resources available. The lack of appropriate infrastructure and equipment to enable hand hygiene performance is one of the most important.

The cultural background and even religious beliefs can play an important role in hindering good practices of hand hygiene. Some practitioners see improving compliance as being about changing human behavior and therefore requires inputs from a wide range of specialists including behavioral, psychological and social science. So to improve the compliance rates it is important to follow a planned and step wise approach to the development of interventions, using insight from behavioral science (Bartholomew et al, 2006). The table below outlines the main factors affecting hand hygiene compliance.

Table 1. The main factors affecting compliance with hand hygiene.

\begin{tabular}{|l|l|}
\hline Material Factors & $\begin{array}{l}\text { - Convenient and accessible hand hygiene facilities } \\
\text { e.g. fast-drying hand rubs, no-touch sinks, hand rubs } \\
\text { at patientsbedsides, hand rubs outside patients } \\
\text { rooms, hand rubs on the patients_notes trolley } \\
\text { during a ward round }\end{array}$ \\
- Preparations which do not cause skin irritation
\end{tabular}


Texila International Journal of Clinical Research

Volume 3, Issue 2, Dec 2016

\begin{tabular}{|c|c|}
\hline & - Preparations which are aesthetically acceptable \\
\hline Behavioural and social factors & $\begin{array}{l}\text { - Perceived danger for carer of omitting hand } \\
\text { hygiene } \\
\text { Practices } \\
\text { - Perceived benefit for dependent or patient } \\
\text { - Concern for third party opinion e.g. peer pressure, } \\
\text { conforming to social ideals } \\
\text { - Gender }\end{array}$ \\
\hline Factors in a healthcare institution & $\begin{array}{l}\text { - Avoid overcrowding and understaffing } \\
\text { - Rewards and sanctions } \\
\text { - Promotion of a positive culture for hand hygiene } \\
\text { - Provision of reminders for hand hygiene } \\
\text { - Encourage active participation in the design of } \\
\text { handhygiene programmes at all levels }\end{array}$ \\
\hline
\end{tabular}

Source (WHO, 2009)

Generally compliance rates are very low. It is 38.9 for doctors and 61.4 \%for nurses (Gilbert K, Stafford C., Crosby K., Fleming E. and Robert G, 2010) and it is as high as 75\% for nurse to $47 \%$ ( Randle et al, 2010) and also in another study it is 43.4 for nurses while it is low as $12.3 \%$ for doctors (Batista, 2010). So it is apparent that doctor's compliance is generally less than nurses. It may be that doctors have a distinct culture associated with levels of power which means that they can be difficult with respect to accepting change (Brooks \& Brown, 2003). Historically, doctors have been a powerful pressure group and are perceived as being resistant to change, or opposed to threats to their autonomy (Allegranzi \& Pittet, 2009). Similarly, Ferlie and Shortell state that doctors often resist efforts to standardize practices and impose rules, as in complying with effective hand hygiene (Ferlie \& Shortell, 2001).

It may be that the cultural aspect affects doctorssompliance, especially if they know that they were observed. The same findings regarding physicians were observed by Korniewicz. and El-Masri (2010) that they are less likely to be compliant with hand washing guidelines. This low compliance persisted when examined by external accredited body compared to the other health care workers whereas they showed statistically significant improvement in hand hygiene compliance after the visit (Korniewicz. and El-Masri, 2010). But when comparing different groups of physicians, professors showed the highest compliance rate of $78.4 \%$ while residents and graduation students did not achieve more than 50\% compliance. (Gilbert K, Stafford C., Crosby K., Fleming E. and Robert G, 2010).

One research evaluated hand hygiene compliance by day of the week (Monday through Saturday) and time of observation. Increasingly, during the week the compliance rate (Randle. et al, 2010 ) was highest on Monday (96.5\%) compared to Friday (83.7\%). This means that compliance was better during the first part of the week compared to the end of the week. This also applied to studies in industry, showing a declining work output as the work week progresses; suggesting cumulative fatigue to employee morale as a contributing factor (Duggan J., Hensley S., Khuder S., Papadimos T, and Jacobs L, 2008). The study also revealed that compliance rate is higher when observed in afternoon shifts compared to morning shifts.

These above percentages changed when there is intervention like using bed rail antiseptic system: improved from $36.4 \%$ to $51.5 \%$ but declined six months later (Giannitsioti E. et al, 2009). Although in the same study, the percentage in the already existing bed rail antiseptic system wards did not change. However when using contact precautions also does not alter the percentage of compliance much: for nurses it is $61.4 \%$ for patients in contact precaution rooms compared to $57.1 \%$ in noncontact precaution rooms (Gilbert K, Stafford C., Crosby K., Fleming E. and Robert G, 2010). 
The last findings contradicted what was found in the literature which suggested that hand hygiene compliance is somewhat better for patients in contact precaution rooms.

Another finding was that compliance rate is higher among health care workers after doing any procedure for patients to $72.1 \%$ compared to $41.7 \%$ before doing any procedures. (Korniewicz. and El-Masri, 2010). Although, pre procedure hand hygiene intends to protect the patients against infections and maximize risk reduction, whereas post procedure hand hygiene intends to protect health workers and other patients.

As expected, high risk procedures show better compliance than low risk procedures (Korniewicz. and El-Masri, 2010). Also another study examined the effect of feedback intervention of hand hygiene compliance through a control trial group; unfortunately there was no significant effect of this feedback on improving compliance (Marra et al, 16 2008).

Since complying with hand hygiene has a good correlation with behavioural change, therefore examining the potential determinants of hand hygiene compliance is very important. With help of qualitative study. (Erasmus, Brouwer, Beeck, Oenema, Daha., Richardus and Brug, 2009) examined and analysed the behavioural determinants of hand hygiene compliance among different hospital healthcare workers, including physicians, nurses, and medical students. The hand hygiene behaviour of healthcare workers appeared to be motivated by self-protection and a desire to clean oneself after a task that is perceived to be dirty. Nurses and medical students expressed the importance of hand hygiene for preventing crossinfection among patients and themselves, whereas physicians expressed the importance of hand hygiene but also perceived a lack of evidence for the importance of hand hygiene in preventing cross-infection.

Personal beliefs about the efficacy of hand hygiene and the examples set and norms established by senior staff in a hospital are of major importance for hand hygiene compliance. Medical students tend to copy the hand hygiene behaviour of their superiors, leading to noncompliance when they observe noncompliance by others. Physicians mentioned that their noncompliance was associated with a perceived lack of evidence that hand hygiene is effective in the prevention of hospital-acquired infection, which could be an explanation for the inverse correlation found between the level of education and the rate of hand washing compliance (Erasmus, Brouwer, Beeck, Oenema, Daha., Richardus and Brug, 2009).

\section{WHO hand hygiene multimodal improvement strategy}

Based on the evidence and recommendations from the WHO Guidelines on Hand Hygiene in Health Care, a number of components are needed for an effective multimodal strategy for hand hygiene. The WHO multimodal hand hygiene improvement strategy was proposed to translate into practice the WHO recommendations on hand hygiene and this is accompanied by a wide range of practical tools (WHO, 2009) To facilitate the change, this change project used the WHO Hand Hygiene Implementation multimodal improvement strategy (WHO, 2009).

The five essential elements of the strategy include:

- System change: ensuring that the necessary infrastructure is in place to allow health-care workers to practice hand hygiene. Including infrastructures provision, hand washing facilities, alcohol hand rub dispersion, adequate staffing, supplies provision (water, soap, antiseptic), polices \& guide lines setting

-Training and education: providing training for health-care professionals about the importance of hand hygiene and the correct procedure of hand washing and rubbing

-Evaluation \& feedback: monitoring \& evaluation of the system setting; including monitoring of hand hygiene practices (hand washing, alcohol hand rub use, wearing of gloves and giving performance feedback

-Reminders at the workplace: (e.g. posters) reminding health-care workers about the importance of hand hygiene and about the appropriate indications and procedures for performing it. 
-Institutional safety climate: this includes creation of a hand hygiene safety culture with the participation of both individual HCWs and senior managers. It includes: active participation at institutional \& individual levels, and washing priority —allocating money and time.

\section{Summary}

The literature review highlighted the historical context and the main cultural and professional barriers to hand hygiene. The importance of hand hygiene to patient and HCW safety was highlighted and this was also evidenced by the First Global Patient Safety Challenge. Multi-factorial barriers require evidence based multimodal interventions. The five elements of the WHO Hand Hygiene Multimodal Improvement Strategy were described as the main intervention for this change management project.

\section{Methods}

\section{Introduction}

Change has become an ever-present feature of our work environment, and the management of these continuing changes is a key challenge for all managers. The need for change in the health service is now widely recognised - by public, by professions and by government. As Burnes (2000) rightly claims _what almost everyone would like is a clear and practical change theory which explains what changes organisations need to make and how they should make them'. He goes on to explain that instead what is available is a wide range of confusing and contradictory theories, approaches and recipes. McAuliffe (2000) argues therefore that managers should be prepared to adopt a contingency approach - choosing or developing the model to suit the particular situation. Hence, the change agent's quest — should not be to seek out an all-embracing theory but to understand the strengths and the weaknesses of each approach and the situations in which each can best be applied, according to Burnes (2000).

In this chapter, the steps to implement the selected HSE change model (2009) are described; in particular how the WHO multimodal strategy was implemented in the three ICUs

\section{Change model}

For the change to be successful, the HSE change model will be used to drive the project because according to the model background it has been developed to improve the experience of patients and service users, help staff and teams play a meaningful role in working together to improve services and promote a consistent approach to change across the system (HSE, 2009).

The HSE Change Model pays particular attention to the people and cultural aspects of change. Its main vision is that everybody will have easy access to high quality care and services that they have confidence in and staff are proud to provide. (HSE, 2009). It is built on and reflects several core principles:

- Ensuring that the needs of service users and local communities together with the interests of staff are at the core of the change process

- Building integration and a whole-system approach, focusing on the connections, relationships and dependencies between different parts of the system

- Encouraging collaboration between different agencies, local communities, services, teams, professional groupings, trade unions, and between national, area and local levels

- Promoting active engagement and participation of services users, staff and their representatives in the change process

- $\quad$ Placing a particular emphasis on partnership and team working

- $\quad$ Prioritizing long-term sustainable change and improved organizational effectiveness

- Providing for the transfer of knowledge and skill so that the system equips itself to manage change 
- $\quad$ Promoting processes of organizational learning through regular feedback, measurement and evaluation at all stages of the change journey

- $\quad$ Locating the responsibility to manage change at all levels of the system, individual, group and organizational and at local, area and national levels

\section{Steps of implementation of the HSE Change Model}

The HSE Change Model describes the journey of transformation that enables people to move from the current situation to the desired future, in line with a shared vision for change. Figure 1 outlines the HSE Change Model based on the four stages of the project management lifecycle; which are: initiation stage, planning, implementation and mainstreaming. The steps in adopting the HSE Change Model adoption therefore included these four management stages.

\section{Initiation stage: preparing to lead the change}

This is an early preparation and scoping step that is meant to create readiness and a considered case for change, to establish a sense of shared responsibility, and to scope out a solid foundation for successful change. At this step three meetings and two brain storming sessions were carried out followed by a workshop attended by policy makers in the Ministry of Health, the Oral Health department, stakeholders from the selected ICUs and stakeholders from the other ICUs as observors. Under this preparation phase to lead and drive the change the following was done according to HSE change model:

- Identification of what is driving the need for change and the degree of urgency to introduce the hand rub methods besidse increasing compliance towards hand washing. This involved presenting the rationale (drawn from the literature review) regarding the magnitude of health care associated infection and hand hygiene as the most single measure to reduce infection rates. Furthermore, the baseline data about hand hygiene compliance in the three ICUs when done the previous January was not good. All this directed the attention of workshop participants to agree on the project as a priority as soon as possible.

- Clarification of leadership roles descriptions at different levels was written. An authorized team at the and identification of the key influencers and stakeholders at different levels including the HCWs in the selected ICUs was done. This was done through special committee with my assistance and a clear document stating the responsibilities and job senior management level was assigned with clear mandate throughout the whole project. I was the head of the team and this also included the director of oral health department and a representative from human resource department, finance department, quality and safety department, the three ICU's. The main function of this senior team was to design, manage and lead the change process. Further three supervisors were recruited; one for each ICU. The supervisors acted as mid-level managers for each clinic and they were responsible for the day to day follow up of the change project, reporting to me and communicating with the senior team.

- Assessing the readiness and capacity for receiving and accepting the change from the current state to the expected one at the end of the project through a checklist approach and focus group discussions which revealed the need for assistance and support at the level of the ICUs including availing hand rub and hand washing materials, training and education, and reminder posters.

- Attending to organizational politics: I was fortunate that I had held the senior position of Director General of the State Ministry of Health two years previously. So I fully understood the general managerial situation, the political and power dynamics among the ministry and oral health department leaders, and the cultural background and contextual aspects of the stakeholders within the Ministry. All these served as enabling factors for me for better understanding, influence and resolving the problems during the implementation phase.

- Identifying the leverage points and opportunities for change as follows: 
- Strengths : top leaders and managers commitment in the oral health department, wellstructured department and clear policies and managerial hierarchy at all levels of the department.

- Opportunities: top leaders commitment at the level of the Minister and Director General, the oral directorate received ISO 9001:2008 certificate one year ago, and the possibility of allocating more resources from the Ministry to this project

- Weaknesses: Poor compliance with hand washing as assessed in January and affirmed in the focus group discussion; hence concerted action needed (multimodal) to rectify this situation

- Threats: the very short time of the project and pressure to expand the change project to other ICUs immediately even prior to the evaluation of this pilot.

- Performing an initial assessment of the impact of the change. The project impact statement was prepared (see Table 4).

- Outlining the initial objectives and outcomes for the change which was presented as part of the project proposal and agreed on it through the above meetings and workshop.

- Agreeing on the initial resource requirement motivation (including the newly recruited midlevel supervisors): This was done in a meeting involving the supervisors, doctors and nurses of the three ICUs.

- Outlining the initial business case for change-The full proposal document for this change management project was prepared and presented to senior managers.

Table 2. Project impact statement

\begin{tabular}{|c|c|}
\hline How things are now in relation to the issue & $\begin{array}{l}\text { How things should (ideally) be when the } \\
\text { issue has been addressed }\end{array}$ \\
\hline $\begin{array}{l}\text { Behavioural : } \\
\text { health workers } \\
\text { sometimes: } \\
\text { - Wear gloves without Handwashing } \\
\text { • Do not use alcohol hand Rub } \\
\text { - Do not wash hands between patients }\end{array}$ & $\begin{array}{l}\text { Behavioural : } \\
\text { health workers: } \\
\text { - Use alcohol hand rub frequently } \\
\text { - Wash hands or use alcohol hand rub } \\
\text { before putting on gloves and after taking } \\
\text { off gloves } \\
\text { - Wash hands with soap and water when } \\
\text { visibly dirty or soiled }\end{array}$ \\
\hline $\begin{array}{l}\text { Structural: } \\
\text { Handwashing facilities are usually } \\
\text { available but supplies are not available in } \\
\text { some instances }\end{array}$ & $\begin{array}{l}\text { Clinic managers ensure alcohol hand rub } \\
\text { is available in the selected ICUs } \\
\text { Clinic managers ensure soap and clean } \\
\text { running water are available in the selected } \\
\text { ICUs } \\
\text { New policies and guidelines are in place to } \\
\text { demonstrate responsibilities of health } \\
\text { workers in hand hygiene }\end{array}$ \\
\hline Personal: & rsonal \\
\hline
\end{tabular}




\begin{tabular}{|l|l|}
$\begin{array}{l}\text { My role is currently as an interested } \\
\text { observer, and former Director General } \\
\text { I am motivated to make a difference to } \\
\text { improve compliance }\end{array}$ & $\begin{array}{l}\text { I see my role as an engine to ensure clinic } \\
\text { managers and Directorate Head are } \\
\text { motivated to see the change through Also } \\
\text { as a facilitator to help solve problems as } \\
\text { they arise. } \\
\text { I will continue to work with the } \\
\text { Directorate to ensure the change is } \\
\text { sustained }\end{array}$ \\
\hline $\begin{array}{l}\text { Cultural: } \\
\text { Handwashing is currently perceived as not } \\
\text { important and a waste of time and money } \\
\begin{array}{l}\text { Incorrect perception that wearing gloves } \\
\text { replaces hand hygiene }\end{array}\end{array}$ & $\begin{array}{l}\text { Cultural: } \\
\text { Managers spend time and money on } \\
\text { resources for hand hygiene because } \\
\text { everyone agrees it is a priority and it is } \\
\text { worth the time and cost }\end{array}$ \\
\hline
\end{tabular}

\section{Planning stage}

The purpose of planning was to determine the specific detail of the change and to create support for the change process to ensure that people are joining in a concerted effort, with a clear purpose and create a new future for the organization (HSE) It included the following steps:

- Building commitment: This meant that I needed to increase the commitment to the project across the system. Further activities were done aiming at creating a shared sense of vision towards the proposed change. Without commitment and talking the same language especially at the top level, resistance is expected leading to extreme failure of the project. At this stage of the project the full proposal document was presented to the senior leaders and managers exploring the magnitude of health care associated infection, the need for introducing and implementing hand hygiene programs and the detailed activities in the project. Also these issues were communicated to the mid level managers, selected supervisors and stakeholders at the ICUs. Side meetings, one to ones, and sharing some evidence articles was done to communicate the vision and the change rationale. Furthermore, stakeholders capacity building was one of objectives of this work as discussed in the meetings mentioned above.

- Determining the detail of the change: The current situation in the ICU versus the expected future vision was explored. This included the assessment of the current situation against the future vision for change. Feedback of this analysis was delivered to key stakeholders exploring the gap and describing what is needed materials, appropriate system to support the change and specific details for each of the five elements of the WHO multimodal strategy to be applied in the ICUs. The proposed change was to improve hand hygiene compliance among health care workers in ICU s of UAE. This will be done by increasing compliance to hand washing as such, as well as introducing another simple, quick and effective procedure of hand hygiene which is using alcohol hand rub

- Developing the implementation plan- One of the main steps planning the change is developing and agreeing on the implementation plan. The implementation of the WHO multimodal strategy on hand hygiene included five main steps: system change, training and education of healthcare professionals; monitoring of hand hygiene practices and performance feedback; reminders in the workplace and the creation of a hand hygiene safety culture with the participation of both individual HCWs and senior manager (WHO, 2009). A detailed description of each step was written including the time frame for implementation, and roles/responsibilities for each action.

\section{Implementation stage}

This stage focused on implementing and monitoring the implementation/project plan to ensure that it is meeting its purpose and attending to the factors that will assist longer-term sustainability. As 
stated above in the planning phase, the WHO multimodal strategy hand hygiene was planned to be implemented under the following five elements:

- System change

- Intensive care facilities will have sinks for handwashing available in each clinical setting, complete with access to safe running water, soap and disposable towels.

- Refilled water tank available in each clinic as a backup if piped tap water discontinued

- Alcohol based hand rub available at each point of care in the ICUs according to the WHO standards for antimicrobial efficacy and in sufficient quantities.

- Durable, heavy duty and good quality dispensers purchased

- Supplies of good quality examination and sterile surgical gloves were maintained

- Storage system for the above materials with $25 \%$ stock alarm developed. This software system automatically gives alarm when the stock of the above materials in the ICUs reached $25 \%$ asking the manager in charge to deliver materials to the immediately.

- $\quad$ Providing guidelines and standard operating procedures about hand hygiene including guidelines on when to wash hands and when to use alcohol rub. These included _My 5 moments for hand hygiene' (WHO, 2009).

- Training and education. This is one of the crucial elements of the strategy. Also it remains one of the critical success factors of the project. The objective of the training was stated clearly aiming to induce behavioural and cultural change and ensure that competence is deep-rooted and maintained among all staff in relation to hand hygiene (WHO, 2009). The targeted group were selected including senior staff in the oral health department at the highest level, the supervisors and the doctors at the mid level and finally all the other health care workers. Fifteen training sessions were delivered addressing infection control, definitions and impact of health care associated infection, source and ways of infection transmissions, patient safety in general, WHO programmes of patient safety, WHO programmes on hand hygiene including the WHO multi modal hand hygiene improvement strategy and WHO programmes on My 5 moments for hand hygiene'. Other tools apart from the above mentioned sessions are hand hygiene brochures, training films from WHO website and practical sessions demonstrating how to hand wash and how to hand rub.

- Reminders in the work place: These are important tools to remind health care workers in the ICUs about the importance of hand hygiene, the indications and the procedures of hand washing and hand rub. Also these reminders were used to educate patients and visitors. The reminders used in the ICUs are posters and stickers in the clinic walls and points of care. The posters used as a reminder tool are: WHO My five moments for hand hygiene poster, when and how to hand wash poster, and when and how to hand rub poster.

- Evaluation and feedback: The monitoring \& evaluation (M\&E) system was set up, including monitoring of hand hygiene practices and compliance (hand washing, alcohol hand rub use, gloves wearing), knowledge and perception of health care associated infection and system setting. Also giving performance feedback to the stakeholders is very important. The key success indicators are: increase in hand hygiene compliance, improvement in infection control / hand hygiene infrastructures, increase in usage of hand hygiene products, improved perception of hand hygiene and improved knowledge of hand hygiene (WHO, 2009). The evaluation tools will be detailed further in the next chapter.

- Institutional safety climate: The institutional safety climate refers to creating an environment and the perceptions that facilitate awareness-raising about patient safety issues while guaranteeing consideration of hand hygiene improvement as a high priority at all levels. For this to take place, active participation at both the institutional and individual levels, awareness of individual and institutional capacity to change and improve (self-efficacy), and partnership with patients and patient organizations is needed (HSE project as a precious pilot which finds support from everywhere. Moreover, in one of our regular meeting with doctors when talking about sustainability of hand hygiene practices in their ICUs they said that they cannot imagine that they can stop doing or 
practising it especially after they know that they are at risk of acquiring infection also and not just the patients.

\section{Mainstreaming stage}

The purpose of mainstreaming is to focus attention on the success of the change effort and on integrating and sustaining the new ways of working and behaving. This stage also focuses on mechanisms for evaluation and continuous improvement (HSE, 2009). This stage has two steps:

- Making it the way of our business: The purpose of this step is to help people and stakeholders in the project to integrate and practice hand hygiene new behaviours, skills and practices. This was done by acknowledging success from time to time and motivating staff to do more and more during the process. Also, I used to work with the targeted HCWs personally and give them support on their daily activities especially during the first two weeks so as to overcome any resistance to the change process. My support and work with HCWs in ICUs gave me idea about the process and pathways and clear lines of responsibility and accountability for decision-making which is very important to support the process of change.

- $\quad$ Evaluating and learning: Evaluation has been defined as the systematic and structured process of reviewing an experience, determining its worth (HSE, 2009). The objective of this step is to build a system of continuous evaluation and learning from implementation of the project change process. This will build on the M\&E system devised for the change project. The focus is on improving the organisation's readiness to engage in future change and to discontinue any activity that no longer serves the needs of the new organisational reality (HSE, 2009). This is also relevant to mainstreaming adoption within other ICUs and within other curative settings.

\section{Summary}

This change project applied the WHO Multimodal Strategy elements to the implementation phase. In line with the HSE change management model, specific steps were undertaken to initiate the project, plan the change, implement and secure commitment to mainstreaming. The activities and methods described depended on the support of a range of stakeholders within the oral health department and staff in the ICUs, all keen to undertake this change and adopt change as the way we do our business. My own role in this has been as a leader and initiator of change as well as more practically and personally working alongside HCWs in the selected ICUs. My role continues to sponsor the change and adoption of better compliance to hand hygiene and mainstreaming to other settings.

\section{Evaluation}

\section{Introduction}

Evaluation is defined as a method of measuring the extent to which an intervention achieves its intended objectives. Evaluation also involves making judgements about the value of what is being evaluated (Gerrish and Mawson, 2005).

In the health care context, evaluations are generally undertaken for a clinical intervention, care programme or service innovation and it is recognized that new initiatives in patient care and service delivery should be subject to evaluation in order to judge their effectiveness, efficiency, economy and equity (Gerrish and Mawson, 2005, and Lazenbat, 2002). Evaluation provides practical information to help decide whether a development or service should be continued or not. Evaluation also forms an important part of everyday clinical practice in that care provided to individual patients is evaluated in order to inform clinical decisions about the care of that patient.

An essential component of the change project was its evaluation; and this chapter describes the evaluation tools used, the outcomes of the change including the results of pre and post-assessments, and ending with a short summary of the evaluation. 
Texila International Journal of Clinical Research

Volume 3, Issue 2, Dec 2016

\section{Change Indicators}

This change project was evaluated continuously and the change indicators loosely (Table 5) follow the project impact statement defined from the start

Table.3. Projected outcomes of the change project

\begin{tabular}{|c|c|}
\hline Pre intervention & Post intervention \\
\hline 1. Current compliance with hand hygiene practices & 1. Improved Compliance \\
\hline $\begin{array}{l}\text { 1.1.current hand washing practices } \\
\text { 1.2.current use of alcohol hand rub } \\
\text { 1.3.current gloves wearing practices }\end{array}$ & $\begin{array}{l}\text { 1.1.increase hand washing practices } \\
\text { 1.2. increase use of alcohol hand } \\
\text { rub } \\
\text { 1.3. increased gloves wearing }\end{array}$ \\
\hline 2. Current staff attitude \& perception & $\begin{array}{l}\text { 2. Improved staff attitude \& } \\
\text { perception }\end{array}$ \\
\hline $\begin{array}{l}\text { 2.1.priority to hand washing (time \&money) } \\
\text { 2.2.current culture to using gloves as replacement } \\
\text { for hand wash } \\
\text { 2.3.current culture of risk infection transmission }\end{array}$ & $\begin{array}{l}\text { 2.1. high priority to hand washing } \\
\text { 2.2.gloves not replacement for hand } \\
\text { wash culture } \\
\text { 2.3.improved services quality }\end{array}$ \\
\hline 3. Current services quality & 3. Improved services quality \\
\hline $\begin{array}{l}\text { 3.1.current status of service inputs:- infrastructures } \\
\text { (alcohol hand rub) supplies available } \\
\text { *clean water supply } \\
\text { *soap } \\
\text { *antiseptics staff availability }\end{array}$ & $\begin{array}{l}\text { 3.1.improved services inputs } \\
\text { available hand rub } \\
\text { available supplies } \\
\text { * available water supply } \\
\text { * adequate soap } \\
\text { *adequate antiseptics } \\
\text { available staff }\end{array}$ \\
\hline $\begin{array}{l}\text { 3.2.current status of processes staff training staff } \\
\text { motivation \& active engagement M\&E system } \\
\text { setting new polices \& guide lines setting }\end{array}$ & $\begin{array}{l}\text { 3.2. improved services process } \\
\text { trained personnel motivated \& } \\
\text { engaged staff Active M\&E system } \\
\text { Available policies\& guidelines }\end{array}$ \\
\hline $\begin{array}{l}\text { 3.3.current status of outputs } \\
\text { Patient satisfaction } \\
\text { Infection rate }\end{array}$ & $\begin{array}{l}\text { 3.3.improved services output } \\
\text { Satisfied customers } \\
\text { Reduced infection rate }\end{array}$ \\
\hline
\end{tabular}

\section{Evaluation tools}

Two types of evaluation tools were used:

1) Observation checklist to assess the service and compliance with hand hygiene,

2) Focus group discussions to assess attitude and perceptions of health workers.

\section{HCWs compliance observation check list}

\section{Objectives:}

To assess compliance with the opportunity of hand hygiene by healthcare personnel within 3 ICUs

\section{Methodology:}

- The checklist included facility resources: No of HH sinks, officers/ICUs, chairs, number of health care workers by professional category.

- In addition, observation of compliance of HCWs was done without the knowledge of health care workers (using mystery shopper approach). 
- Observers were trained and conducted pilot observation period with members of the project team

- Direct observation was used to monitor compliance using the three measures from WHO my 5 moments tool that were valid for ICUs; before patient contact, after patient contact and after clinical procedure

- Each opportunity was then coded as to whether the individual complied (took the opportunity to wash hands or use alcohol rub as appropriate)

- Participants (HCWs) were classified as dentist/doctor or nurse

- Data collection took place from 8 am to $2 \mathrm{pm}$ in the morning. On average one clinic observation took about an hour and twenty minutes each

\section{Data analysis:-}

- All observed data was recorded manually on standardized sheets

- X2 tests were used to test whether variation in compliance was statistically significant across HCWs types, ICUs, time of day \& type of opportunity

- Logistic regression model was used to test whether there was a relationship between compliance and reminders after adjustment for other covariance's

- Data analysis was conducted using SPSS version 10 and STATA version 9.

\section{Focus Group discussions}

The focus group discussion (FGD) method is a facilitated interview with several people on specific topic or issue to be explored in depth (Bryman \& Bell, 2007) In this project, perceptions of health workers were assesssed pre $\&$ post project through focus group discussions.

\section{Objectives:}

To understand the cultural and behavioral determinants of hand hygiene among health care workers in the three ICUs.

\section{Methods:}

- Participants: we required 6-8 health care workers representing the two professional categories

- Informed consent was taken from each participant

- An experienced facilitator ran the session with an assistant to record the session and take notes. Participants responses were transcribed and proofed

- A presentation on hand hygiene with a review of definitions \& guidelines for hand hygiene was presented to participants of FGDs

- Is there a relation between not performing hand hygiene and risk of cross-contamination, in what way?

- Is hand hygiene necessary before/after patient contact or any procedures (and why)?

- Is hand hygiene necessary before/after performing any procedures (and why)?

- Is hand hygiene necessary before /after touching patient surrounding (and why)?

- Is hand hygiene necessary after gloves removal (and why)?

- Could gloves be used as replacement of hand hygiene?

- In your opinion, what are possible reasons for non-compliance.

-Probing questions on possible reasons: Poor training: lack of knowledge of the importance of hand hygiene in preventing infections and lack of understanding of the appropriate hand hygiene technique

-Staff shortage, work overload/time constraints

-Absences of institutional commitment to hand hygiene/lack of role model

-Difficult access to points of conventional hand hygiene

-Non-compliance should be aggressed (possible solutions)

- FGDs duration lasted on average about an hour. Each FGDs meeting was followed by a debriefing session among the facilitator and study assistant and myself as project lead.

\section{Outcomes of change}

- The following results were found three weeks into the intervention and 3 weeks post-intervention The change was initiated in March 2011 (baseline). The first assessment of compliance took place 
Texila International Journal of Clinical Research

Volume 3, Issue 2, Dec 2016

three weeks later and the post intervention assessment was undertaken in May 2011. Longer evaluation was not possible due to the short time of the change project

Table 4. Rate of compliance with hand hygiene by healthcare workers

\begin{tabular}{|c|c|c|c|}
\hline \multirow[t]{3}{*}{ Variable } & \multicolumn{3}{|c|}{ Compliance rate } \\
\hline & At base line & $\begin{array}{l}\text { 3weeks into the } \\
\text { intervention }\end{array}$ & $\begin{array}{l}\text { 3weeks post } \\
\text { intervention }\end{array}$ \\
\hline & No (\%) & No (\%) & No (\%) \\
\hline \multicolumn{4}{|l|}{ 1. Intensive care } \\
\hline Medical ICU & $17 / 41(41 \%)$ & $32 / 56(57 \%)$ & 72/76 (97\%) \\
\hline cardiac ICU & $11 / 20(55 \%)$ & $50 / 72(69 \%)$ & $94 / 100(96 \%)$ \\
\hline Neonatal ICU & $10 / 25(40 \%)$ & $26 / 32(\% 81)$ & $30 / 32(94 \%)$ \\
\hline \multicolumn{4}{|l|}{ 2. Professional Category } \\
\hline Doctors & $14 / 45(31 \%)$ & $32 / 70(46 \%)$ & 96/104 (92\%) \\
\hline Nurses & $24 / 41(59 \%)$ & $76 / 90(84 \%)$ & $100 / 104(96 \%)$ \\
\hline \multicolumn{4}{|l|}{ 3. Hand hygiene method } \\
\hline Hand Wash (soap \& water) & $38 / 86(44 \%)$ & $82 / 160(51 \%)$ & 126/208 (61\%) \\
\hline Hand Rub & & $69 / 160(43 \%)$ & $181 / 208(87 \%)$ \\
\hline \multicolumn{4}{|l|}{ 4. Hand hygiene Indication } \\
\hline $\begin{array}{l}\text { Before patient touch/any } \\
\text { procedures }\end{array}$ & $17 / 40(43 \%)$ & $54 / 80(68 \%)$ & 99/104 (95\% \\
\hline $\begin{array}{l}\text { After patient touch/any } \\
\text { procedures }\end{array}$ & $21 / 46(46 \%)$ & $54 / 80(68 \%)$ & 97/104 (93\%) \\
\hline Total (overall) & $38 / 86(44 \%)$ & $108 / 160(68 \%)$ & 196/208 (94\%) \\
\hline
\end{tabular}

The hand hygiene compliance of healthcare workers was monitored in the three Intensive Care Areas at base line, at 3weeks after starting the intervention and at 3 weeks post intervention with a total 454 hand hygiene opportunities and over all hand hygiene compliance was found to be $75 \%$.

Hand hygiene showed gradual improvement with time, starting from $44 \%$ at base line compared to $68 \%$ at 3 weeks and $94 \%$ at 3 weeks post intervention successively (Table 6).

\section{Hand hygiene compliance}

The escalating trend of hand hygiene compliance rate across the different assessment periods remained valid for the three ICUs (Figure 2). At base line, the compliance rate for the 3 ICUs ranged between $40-55 \%$, which increased to $57-81 \%$ at 3 weeks after starting the intervention and reached the peak with $94-97 \%$ at 3 weeks post intervention.

The hand hygiene compliance of all ICU s showed statistically significant difference $(\mathrm{p}<0.05)$ between the base line data and3 weeks after dat difference starting. There is also stastically between the 3 weeks and 3 significant first weeks intervention and also between base line and post 3 weeks intervention $(\mathrm{p}<0.05)$ 
There was no statistical difference between ICUs in hand hygiene (Table 5); neither at baseline (41\%, 55\% and 40\%) nor post intervention (97\%, 96\% and 94\%) ( $\mathrm{P}>0.05)$.

Figure.2 Rate of compliance with hand hygiene in Intensive care areas:

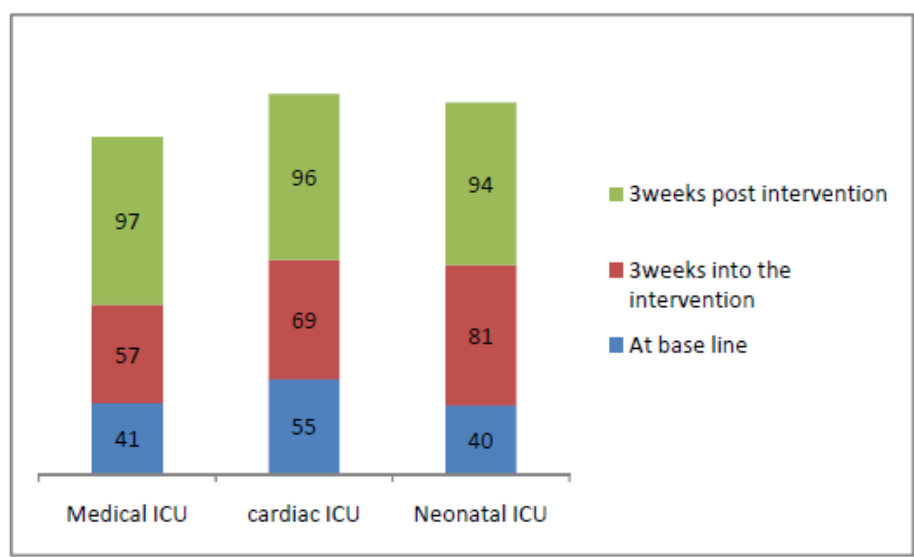

\section{Hand hygiene compliance by professional category}

Compliance with hand hygiene showed statistically significant differences $(\mathrm{P}<0.05)$ between health workers categories at all assessment periods (Figure 3). Nurses scored better compliance (59 \% at base line, $84 \%$ at 3 weeks after starting the intervention and $96 \%$ at 3weeks post intervention assessment), compared to Doctors (31\% at base line, $46 \%$ at 3 weeks after starting the intervention and $94 \%$ at 3 weeks post intervention).

The hand hygiene compliance for both doctors and nurses, showed escalating trends with statistically significance variation $(\mathrm{P}<0.05)$ across the different assessment periods (base line, the first 3 weeks and 3 weeks post intervention).

Figure.3. Hand hygiene compliance by professional category:

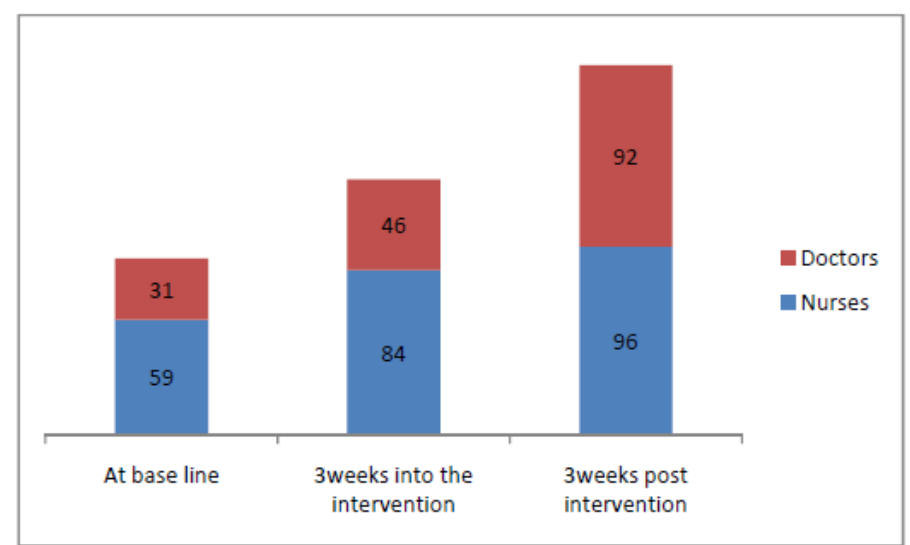

\section{Hand hygiene compliance per hand hygiene method}

The base line assessment of hand hygiene method showed low compliance (44\%) with hand washing, while at that time the alcohol hand rub had not yet been introduced in the health care facilities.

The use of water and soap was the preferred hand hygiene method (51\% compliance) compared to alcohol hand rub (43\% compliance) 3 weeks after starting the intervention (Figure 4). Yet, this preference at 3 weeks post intervention shifted to favor the alcohol hand rub (87\% compliance) compared to water and soap hand washing (69\% compliance). 
The increasing hand hygiene compliance patterns across the different assessment periods showed statistically significant variation $(\mathrm{P}<0.05)$ both for hand washing $(51 \%$ at the first 3 weeks intervention compared to $61 \%$ at 3 weeks post intervention) as well as for alcohol hand rub ( $43 \%$ at the first 3 weeks intervention compared to $87 \%$ at 3 weeks post intervention).

Figure.4. Hand hygiene compliance per hand hygiene method

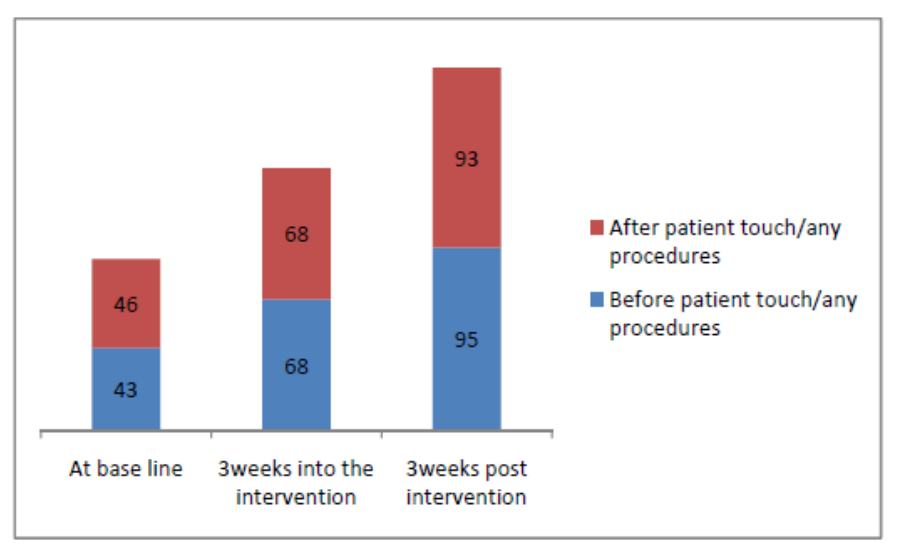

\section{Summary}

Hand hygiene showed rapid improvement from $44 \%$ at base line to $68 \%$ in the first 3 weeks and $94 \% 3$ weeks post intervention. There was no statistically significant difference $(\mathrm{P}>0.05)$ between the 3 ICUs in this respect. There was no difference in hand hygiene before touching patients compared to after patients during all assessment periods.

Compliance to hand hygiene showed statistically significant differences $(\mathrm{P}<0.05)$ between health workers categories in all assessment periods with doctors scoring better compliance compared to nurses. The use of water and soap started off as the preferred method of hand hygiene, yet this preference shifted to favor alcohol hand rub post-intervention at $87 \%$ (compared to water and soap hand washing at $69 \%$ ).

\section{Discussion \& conclusion}

\section{Introduction}

The evaluation demonstrated promising results from the intervention in the three intensive care units. In this chapter, the strengths and limitations of the project are discussed. The implications from this change project for improving hand hygiene are also presented with recommendations for future projects.

\section{Strengths \& limitations of the project:}

Strong leadership commitment was the driving strength in this project both at the level of the Health care Authority and in the Health directorate. Moreover, a well-structured oral health department and clear policies and managerial hierarchy was another strength.

Implementing the change model, with new policies and guidelines and continuous follow up and evaluation mechanisms has proved to be a real challenge due to resistance to change and tolerance to the existing status quo. There was also initial difficulty of communication between management and staff in the selected ICU s, but this was overcome later.

Although more resources were reallocated to this project, still resources are a limiting factor for sustainability. Adding to this shortage of staff especially trained nurses have proved to be real threats to the project implementation. 


\section{Implications of the change project for management}

\section{Overall hand hygiene compliance}

At base line, the overall hand hygiene compliance was found to be below the expectation reporting a rate as low as $44 \%$, which is below the hand hygiene compliance reported in UK study at 2010 by Batista, (55.4\%) and it is also far below hand hygiene compliance reported in studies conducted at Canada and Italy (76\%) (Batista, 2010)

Yet, the data showed considerable and steady progress in overall compliance in the first 3 weeks of intervention with $68 \%$ hand hygiene compliance and 3 weeks post intervention compliance reaching up to $94 \%$. This post intervention compliance level, is far better than the previously mentioned studies.

\section{Hand hygiene compliance per professional category}

The baseline data showed that the hand hygiene compliance of both doctors and nurses were below the expectations at 59\% and $31 \%$. Yet, the post intervention compliance data showed considerable improvement in both doctors and nurses compliance particularly at 3 weeks post intervention (96\% for doctors and $92 \%$ for nurses).

It is well documented in published literature that doctors were found to be more resistant to change and to have poor compliance with infection prevention and control standard (Randel, Clarke \& Storr, 2006) while nurses compliance were known to be higher than doctors (Hugonnet, Prenger \& Pittet, 2002) which could possibly be attributed to doctors'

distinct culture, and their opposing attitude to what they consider as a threat to their autonomy (Randle, Arthur \& Vaughar, 2010). Yet, on the contrary to the above mentioned literature, doctors compliance was found to be better than that of nurses at base line (59\% compared to 31\%), at the first 3 weeks of intervention ( $84 \%$ compared to $46 \%$ ), and at 3 weeks post intervention ( $96 \%$ compared to $92 \%)$.

\section{Hand hygiene compliance per hand hygiene method}

Batista et al 2010, found that hand washing method for hand hygiene was the preferred method in comparison to alcohol hand rub. Yet, the 3 weeks post intervention data showed a different picture as the preference shifted to favor the alcohol hand rub method (87\%) compared to hand wash method (69\%).

\section{Hand hygiene compliance per hand hygiene indication}

It is documented in the published evidence that healthcare workers tend to wash their hands according to their needs (Btista et all, 2010) and thus they tend to comply more with hand hygiene after patient contact compared with their compliance before patient contact (Randle, Arthur \& Vaughar, 2010). Yet, the hand hygiene compliance at all assessment periods (base line, the first 3 weeks of intervention and 3weeks post intervention) showed no statistical difference for opportunities before patient contact compared to those after patient contact.

\section{Recommendations for future improvement}

1. Since the implementation of intervention proved to be beneficial, then the institutionalization and systematic \& sustainable application of these changes might have its rewards in increasing hand hygiene compliance and reduce nosocomial infection transmission in other healthcare settings.

2. Nurses were found to have less compliance in comparison to doctors, the adoption of intervention \& programs specially targeting such an important professional category might provide a good chance of success to increase hand hygiene compliance in health care facilities

3. The systematic and wide application of alcohol hand rub should be encouraged and nested at the top policy makers priority list 
Texila International Journal of Clinical Research

Volume 3, Issue 2, Dec 2016

4. Further research should be adopted to investigate the hand hygiene determinants and to verify the observed association between the implemented interventions and the improvement of hand hygiene compliance in other intensive care areas and other settings.

\section{Reflections on the project}

During the implementation of the project and while I am introducing alcohol hand rub, I faced with strong resistant in one of the clinic. So I decide to pay more attention to healthcare workers in this ICU s by increase training, education and more supervision. Later I discovered that the doctor in charge in the clinic has his own religious concern about using alcohol. I know that Alcohol use is prohibited in Islam or considered an offence requiring a penance because it is considered to cause mental impairment. But that if you drink it or used it in any type of food. This concern took a lot of time from me to conscience him and delayed the intervention for some time in that clinic.

\section{Conclusion}

The hand hygiene compliance rate showed gradual improvement over time, starting from below standard hand hygiene at base line assessment (44\%), to reach its maximum at 3 weeks post intervention assessment (94\%). The introduction of alcohol hand rub was particularly successful and it became the preferred hand hygiene method. These results indicate that the systematic application of the change model and WHO multimodal strategies is associated with improvement in hand hygiene compliance of $l$ healthcare workers and thus it could result in a sustained positive impact. Further work is needed to understand and improve the poorer compliance of doctors compared to nurses.

\section{References}

[1] Ahmed QA et al. (2006). Muslim health-care workers and alcohol based handrubs. Lancet, 367:10251027.

[2] Allegranzi B et al (2008). The burden of hospital-acquired infections (HAI) in developing countries: a systematic review. Poster presented at: 48th Interscience Conference on Antimicrobial Agents and Chemotherapy/46th Annual Meeting of the Infectious Diseases Society of America, Washington, DC, abstr. K4106

[3] Allegranzia B., Storra J., Dziekana G., Leotsakosb A., Donaldsonb L., Pitteta D (2007). The First Global Patient Safety Challenge - Clean Care is Safer Care: from launch to current progress and achievements. Journal of Hospital Infection: 65(S2) 115-123.

[4] Barbacombe J., (2004). Back to the basics - hand washing. Geriatric Nursing 25, 90-92.

[5] Bartholomew LK, Parcel GS, Kok G, Gottlieb NH. Planning health promotion programs: an intervention mapping approach. San Francisco, CA: Jossey-Bass, 2006.

[6] Bhalla A, Aron DC, Donskey CJ (2007). Staphylococcus aureus intestinal colonization is associated with increased frequency of S. aureus on skin of hospitalized patients. BMC Infectious Diseases, 7:105.

[7] Bischoff W, Reynolds T, Scssler C, Edmond M, Wenzel R.,(2000). Hand washing compliance by health care workers: the impact of introducing an accessible, alcohol-based hand antiseptic. Arch Intern Med ; 160:

1017-21 washing compliance by health care workers: the impact of introducing

[8] Bjerke NB., 2004. The evolution: hand washing to hand hygiene guidance.

[9] Critical Care Nursing Quarterly, 27:295-307.

[10] Boyce JM, Pittet D. (2002). Guideline for hand hygiene in healthcare settings. Recommendations of the Healthcare Infection Control Practices Advisory Committee and the HICPAC/ SHEA/APIC/IDSA Hand Hygiene Task Force. Morbidity and Mortality Weekly Report, 51:1-45.

[11] Brooks I, Brown RB., (2003). The role of the ritualistic ceremonial in removing barriers Between subcultures in the National Health Service. J AdvNurs; 38: 341e352.

[12] Burnes, B. (2000). Managing Change. A Strategic Approach to Organisational Dynamics, 3rd Edition. Essex, England: Prentice Hall. 
[13] Centres for Disease Control and Prevention, (2002). Guideline for Hand Hygiene in Health-Care settings: Recommendations of the Healthcare Infection Control Practices Advisory Committee and the HICPAC/SHEA/APIC/IDSA Hand Hygiene task Force. MMWR ; 51 (No. RR-16):1—48.

[14] Cosgrove SE (2006). The relationship between antimicrobial resistance and patient outcomes: mortality, length of hospital stay, and health care costs. Clin Infect Dis ; 42(Suppl. 2):S82eS89.

[15] Duggan J., Hensley S., Khuder S., Papadimos T. And Jacobs L.(2oo8). Inverse correlation between level of professional education and rate of hand washing in a teaching hospital. Infection Control and Hospital Epidemiology vol; 29, no. 6:534-538.

[16] Dumpis U et al (2003). Prevalence of nosocomial infections in two Latvian hospitals. Eurosurveillance, 8:73-78.

[17] Encyclopaedia Britannica Online. www.britannica.com.

[18] Erasmus V., Brouwer W., Beeck, F., Oenema A., Daha J., Richardus H., Brug J., 21 ( 2009). A Qualitative Exploration of Reasons for Poor Hand Hygiene. Among hospital workers: Lack of Positive Role Models and of Convincing Evidence that Hand Hygiene Prevent Cross Infection. Infection Control and Hospital Epedimiology30:415-419

[19] Ferlie E, Shortell S., 2001. Improving the quality of health care in the United Kingdom and the United States: a framework for change. Milbank Q;79:281e315.

[20] Fridkin S et al.(1996): The role of understaffing in central venous catheter- associated bloodstream infections. Infection Control and Hospital Epidemiology, 17:150-158. 


\title{
Review of updates on the prevalence and epidemiology of Acetaminophen poisoning in the United States and the United Kingdom
}

\author{
Article by Njoku Amara Chiemezie, L. \\ M.Sc Clinical Research, Texila American University, Nigeria \\ Email: deepwatersamara@gmail.com
}

\begin{abstract}
Paracetamol was licensed for assumedly safe worldwide usage in 1955 and is debatably the most common form of pain relief, headache palliative and hyperthermia medication used in both pediatric and adult populations in the Americas. Acetaminophen as it is commonly referred to in the United States is a component of very numerous treatment protocols for a wide range of ailments worldwide. Its patency permits has given rise to newer names in various countries using such names as paracetamol and panda. Although fatal and nonfatal liver based injuries, (some microscopic and others macroscopic) have been reported since 1966 due to overdose medications (usually self inflicted) due to self medications. Despite these problems, it's a reasonably safe drug when taken within its therapeutic dose range. Paracetamol poisoning is portrayed as a foremost causative factor in the emergence of acute liver damage in the Americas. It is note worthy that certain factors tend to favor the emergence of paracetamol induced liver damage like chronic use of alcohols, certain enzyme inducing drugs, and associated liver parenchymal diseases even under the circumstances of a normal therapeutic usage of the drug- paracetamol / acetaminophen. Certain questions beg for answers. Are there new findings from decades of pharmacovigilance? Is there a possibility of genetic mutations observed? Is paracetamol still steadfastly safe as portrayed in pregnancy as has been documented since 1966? These are some questions seeking updates.
\end{abstract}

Keywords: Hepatic failure; drug poisoning; pharmacovigilance; epidemiology; prevalence; hyper medication

\section{Introduction}

Acetaminophen is an analgesic and an antipyretic drug. It has ubiquitous applications and is readily available worldwide with no less greater potential for toxic effects as compared to other common pain relief medications like ibuprofen and aspirin ${ }^{[1][3 a]}$ but review of updates show it indeed has a much higher potential for events of hyper medication. According to Larson et al ${ }^{[2]}$ it is said to be the commonest direct etiologic agent in cases of acute hepatic failure in the United States and the United Kingdom. In England and Wales an estimate of over 40,000 cases of acetaminophen poisoning occurred from 1989 to 1990, (mortality of over $0.39 \%$ ) ${ }^{[3 b]}$. It is estimated that average of 175 deaths and 16 to 19 liver transplants occur as a result of poisoning each year in England and Wales.(See fig.1.) Acetaminophen overdose results in more visits to emergency units in the US than overdose of any other pharmacological substance, over 50,000 emergency room visits, 2,100 hospitalizations, and over 452 deaths due to acute liver failure per year. ${ }^{[4][5] .}$ A study of reported cases of acute liver failure in the USA noted that acetaminophen was the culprit in over $40 \%$ of all cases in adults, and over $23 \%$ of cases in under fives age groups. As at May 18, 2016, it is stated that acetaminophen poisoning has overtaken viral hepatitis as the leading cause of acute liver failure in the United States. (emedicine.medscape.com/article/820200-overview) 
Texila International Journal of Clinical Research

Volume 3, Issue 2, Dec 2016

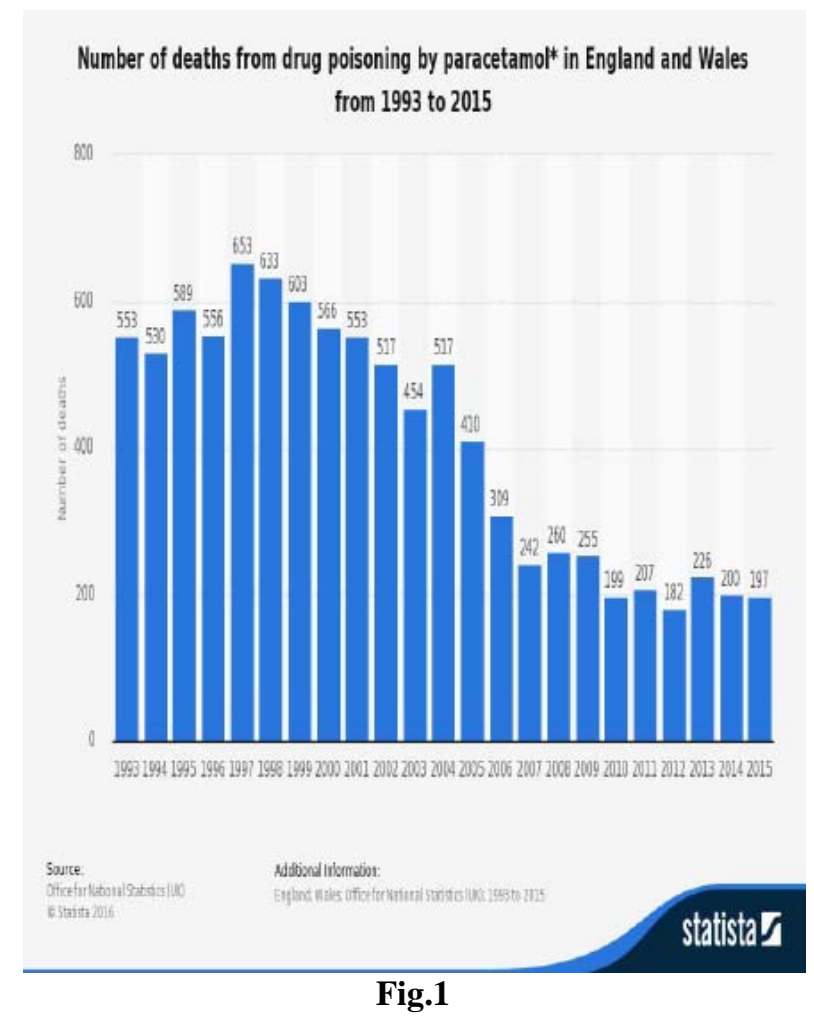

\section{Hyper dosages and suicide}

Paracetamol is one of the leading self prescribed drugs in cases of voluntary hyper medications. ${ }^{[5][6]}$ In the UK, paracetamol is the most common culprit in hyper dosage, responsible for more than $40 \%$ of oral intake of poisons admissions to the hospital and associated with an approximated over one hundred and fifty deaths per year.(http://bestpractice.bmj.com/bestpractice/monograph/337/resources/references). However, the incidence of poisoning in the US was above seventy thousand acetaminophen-related cases documented at the American Association of Poison Control Centers in 2006, with cases of young females out weighing all other gender or age based demarcations. There were approximately over 950 cases of severe paracetamol toxicity and over 97 cases of paracetamol-related deaths. (See fig.2\&3). The drug was also implicated as a drug of choice in voluntary self harm schemes amongst teenagers in the most developed economies of the world.

In a majority of developing countries, like Nigeria, the incidence of paracetamol poisoning rarely approximates that of the United States or United Kingdom rather it seems toxicities due to Tramadol and other narcotic agents hold sway amongst the teenage group 14 - 18years. Paracetamol overdose can result from paracetamol-only preparations but also from special combinations of paracetamol with other drugs like the popular combifen and cafégot brands in Nigeria. These paracetamol-containing preparations can be either prescription medication or nonprescription preparations. Over 49 million people are said to use paracetamol or paracetamolcombination products each 7day period in the USA.

There is documentation of the presentation of stages of clinical debility over hours in any case of acetaminophen poisoning. Within the first twenty four hours of ingestion, the victim will experience excessive sweating (diaphoresis), nausea and vomiting. There may be an ironical improvement of symptoms over the following forty eight hours but with the development of right upper quadrant pain. As from the $72^{\text {nd }}$ hour to the $96^{\text {th }}$ hour post ingestion without medical intervention, liver functions begin to fail and gastrointestinal problems may ensure. However, from $4^{\text {th }}$ day up to 2 weeks post ingestion, patient's liver problems may resolve in over $70 \%$ of all victims. The Rumack-Matthew normogram is a device for assessing probability of hepatotoxicity in acute overdose. (See fig.3.) 


\section{Self prescription}

Other important factors in the etiology of over dose of acetaminophen are homemadeprescribed regimen for all nature of pains, emergency department patients overuse of nonprescription pain killers, dental pain patients, the use of combination regimen duplicated in various products which may contain and provide increased quantities of acetaminophen unwittingly can lead to unintended overdose, and of course the vascular crisis of sickle cell anemia.

There are an increasing number of research reports of therapeutic paracetamol poisoning relating to the use of intravenous paracetamol in both the adult and pediatric age groups ${ }^{[7][8][9] .}$ Acetaminophen is available in both fast-release and long duration-release forms. The therapeutic dose is 10 to 15 milligram per kilogram per dose in children less than five years and 325 to 1000 milligram per dose in adults, given 4-6 times a day, with a maximum recommended daily dose of 80 milligram per kilogram in children or 4 gram in adults.

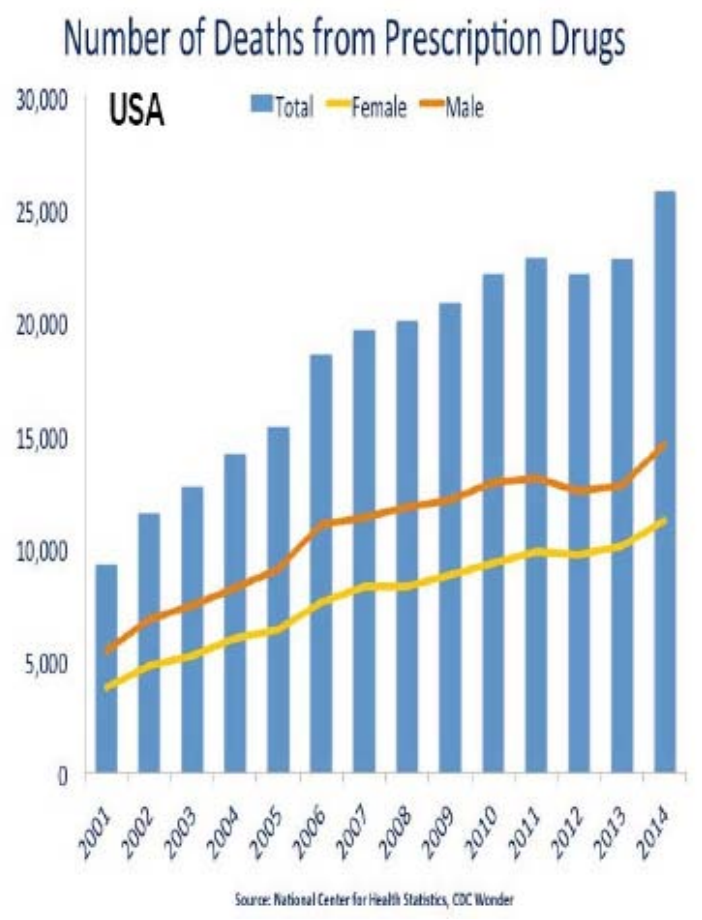

Fig.2. 
Texila International Journal of Clinical Research

Volume 3, Issue 2, Dec 2016

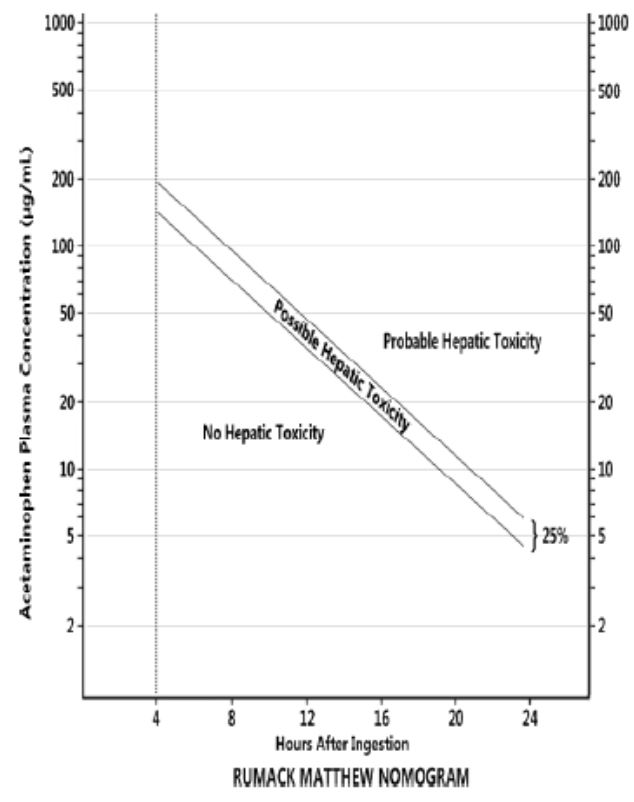

Fig.3*

*(Adapted from Rumack BH, Matthew H. Acetaminophen poisoning and toxicity. Pediatrics1975; 55(6):871-876.)

\section{Pharmacogenetics}

Baseline glutathione levels are an important consideration in determining pharmacogenetics which definitely varies per person. It is important to note that toxicity is less likely to result from a single dose of less than $150 \mathrm{mg} / \mathrm{kg}$ in a child or 7.5 to $10 \mathrm{~g}$ for an adult. However it is more likely to occur with single ingestions greater than $250 \mathrm{mg} / \mathrm{kg}$ or those greater than $12 \mathrm{~g}$ over a 24hour period. Noteworthy is the fact that $97 \%$ of all patients who ingest doses in excess of 350 milligram per kilogram develop severe liver toxicity unless appropriately treated. Acetaminophen exhibits $100 \%$ complete absorption from the intestines. Peak serum concentrations occur between $30^{\text {th }}$ minute and the $120^{\text {th }}$ minute post oral ingestion of therapeutic regime.

Factors causing delays in attainment of peak serum concentrations include co-ingestion of anticholinergic, co-use of morphine, codeines, tramadol and also the use of long duration release forms of the drug. Elimination half-lives range from 60minutes to 240 minutes for all paracetamol preparations, but elimination phase may be delayed in onset for extended-release preparations due to nature of its formulations. Longer half-lives greater than 240 minutes have been observed in patients with liver failure. The casualty rate from acetaminophen overdose worsens 48 hours after ingestion, reaches a peak on day 4 , and then eases off. The zenith indicator of worsening prognosis is the presence of acidemia. A mortality rate of over $92 \%$ without liver transplant was reported in patients whose $p H s$ was less than 7.30. Other indicators of poor prognosis are renal failure, liver encephalopathy, prothrombin time elevation, blood lactic acid elevation and low

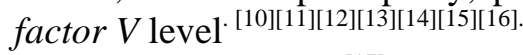

Suarez-Kurtz et al ${ }^{[17]}$ published an article in 2014 proving that ethnicity played a major role in determining adverse effects to acetaminophen. Ward et al., $2001^{[18]}$ carried out a research linking degree of adverse reactions or toxicities to certain nutritional deficiencies.

\section{Pharmacovigilance}

By August 2013, the United States FDA had issued a warning on the reporting of a skin dyscrasia directly linked to the drug acetaminophen as discovered by ongoing pharmacovigilance protocols. This was a side effect originally confined to allergies to non steroidal antiinflammatory agents. FDA issues a safety alert to the public on $1^{\text {st }}$ august 2013 . By $14^{\text {th }}$ January, 2014, FDA directed all doctors and pharmacists to desist from prescribing acetaminophen or any combined therapy of more than three hundred and twenty five milligrams of the drug. Major 
manufacturers of acetaminophen products with dosages more than 325mg acetaminophen have stopped marketing same in the United States by $28^{\text {th }}$ of March, 2014. Latest reviews as at $1^{\text {st }}$ September 2015 has questioned the safety on continued use of acetaminophen as a routine pain medication during pregnancy. Source ( $w w w . f d a . g o v)$.

\section{Legislative interventions.}

Keith Hawton et al ${ }^{[19]}$ published (2013) in the British medical journal assessing the long term effect of a legislation by the UK parliament seeking a reduction in size of paracetamol packs as an intervention to curb the rising incidence of paracetamol poisoning. The law was passed in September 1998. The research stated that there was $43 \%$ reduction in deaths associated with acetaminophen poisoning in over 11years post introduction of the legislation in the United Kingdom. (See fig.3).
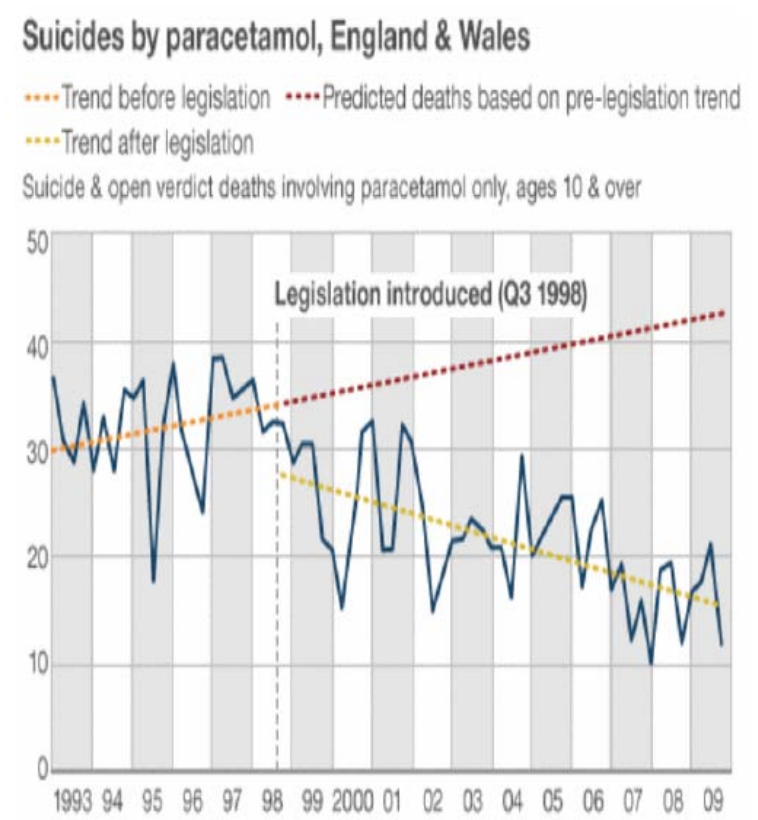

Source: BMJ

Fig.4

\section{Methods}

The research method was a Meta-analysis type, collating and analyzing data from online scientific data bases, abstract guides and references, content analysis, published articles, medical textbooks and research materials from Universities.

\section{Results and conclusions}

The issue of acetaminophen poisoning is an ever present challenge in our world. As long as acetaminophen remains an over the counter medication around the world, the prevalence rates of its toxicity will remain alarmingly high. The pharmacovigilance protocols in the United States had received updates in emergence of new adverse effects of the drug and had issued an alert to its healthcare professionals. The United Kingdom issued a legislation which seemed to have a tremendous positive effect on the prevalence of the drug poisoning over a period of 11 years. The continual surveillance of Acetaminophen in the United States and the United Kingdom is commendable and should be emulated by the under developed countries as a standard practice. The research regarding safety of paracetamol in pregnancy is still ongoing although FDA of the United States had issued a query on September, 2015 regarding the matter. 
Texila International Journal of Clinical Research

Volume 3, Issue 2, Dec 2016

\section{References}

[1]. Efficacy and safety of acetaminophen vs. ibuprofen for treating - NCBI

[2]. www.ncbi.nlm.nih.gov/pubmed/15184213 by DA Perrott et al- 2004 -.

[3]. Larson AM, Polson J, Fontana RJ, Davern TJ, Lalani E, Hynan LS, Reisch JS, Schiødt FV, Ostapowicz G, Shakil AO, Lee WM; Acute Liver Failure Study Group. (December 2005). Acetaminopheninduced acute liver failure: results of a United States multicenter, prospective study. Hepatology (Baltimore, Md.) 42 (6): 1364-72.

[4]. Sheen C, Dillon J, Bateman D, Simpson K, Macdonald T (2002). "Paracetamol toxicity: epidemiology, prevention and costs to the health-care system". QJM: monthly journal of the Association of Physicians 95 (9): 609-19. doi:10.1093/qjmed/95.9.609. PMID 12205339. 3b. Buckley N, Eddleston M (December 2005). "Paracetamol (acetaminophen) poisoning". Clinical evidence (14): 1738-44. PMID $16620471 \ldots$

[5]. Gunnell D, Murray V, Hawton K (2000). "Use of paracetamol (acetaminophen) for suicide and nonfatal poisoning: worldwide patterns of use and misuse". Suicide \& life-threatening behavior 30 (4): 313-26. PMID 11210057.

[6]. Hawkins LC, Edwards JN, Dargan PI (2007). "Impact of restricting paracetamol pack sizes on paracetamol poisoning in the United Kingdom: a review of the literature". Drug safety: an international journal of medical toxicology and drug experience 30 (6): 465-79.doi:10.2165/00002018-20073006000002. PMID 17536874.

[7]. Khashab M, Tector AJ, Kwo PY (March 2007). "Epidemiology of acute liver failure". Current gastroenterology reports 9 (1): 66-73. doi:10.1007/s11894-008-0023-x. PMID 17335680.

[8]. Lee WM (July 2004). "Acetaminophen and the U.S. Acute Liver Failure Study Group: lowering the risks of hepatic failure". Hepatology 40 (1): 6-9. doi:10.1002/hep.20293. PMID 15239078.

[9]. Davidson DG, Eastham WN. Acute liver necrosis following overdose of paracetamol. Br Med J 1966; 2:497.

[10]. Seeff LB, Cuccherini BA, Zimmerman HJ, et al. Acetaminophen hepatotoxicity in alcoholics. A therapeutic misadventure. Ann Intern Med 1986; 104:399.

[11]. Zimmerman HJ, Maddrey WC. Acetaminophen (paracetamol) hepatotoxicity with regular intake of alcohol: analysis of instances of therapeutic misadventure. Hepatology 1995; 22:767.

[12]. Maddrey WC. Hepatic effects of acetaminophen. Enhanced toxicity in alcoholics. J Clin Gastroenterol 1987; 9:180.

[13]. Whitcomb DC, Block GD. Association of acetaminophen hepatotoxicity with fasting and ethanol use. JAMA 1994; 272:1845.

[14]. Schiødt FV, Rochling FA, Casey DL, Lee WM. Acetaminophen toxicity in an urban county hospital. N Engl J Med 1997; 337:1112.

[15]. Benson GD. Hepatotoxicity following the therapeutic use of antipyretic analgesics. Am J Med 1983; 75:85.

[16]. Watson WA, Litovitz TL, Klein-Schwartz W, et al. 2003 annual report of the American Association of Poison Control Centers Toxic Exposure Surveillance System. Am J Emerg Med 2004; 22:335.

[17]. Lee WM. Acetaminophen and the U.S. Acute Liver Failure Study Group: lowering the risks of hepatic failure. Hepatology 2004; 40:6.

[18]. Pharmacogenomic implications of population admixture: Brazil as a model case.

[19]. Suarez-Kurtz G, Paula DP, Struchiner CJ

[20]. Pharmacogenomics. 2014 Feb; 15(2):209-19.

[21]. Ward R. M., Bates B. A., Benitz W. E., Burchfield D. J., Ring J. C., Walls R. P., et al. (2001). Acetaminophen toxicity in children. Pediatrics 108 1020-1024 10.1542/peds.108.4.1020 [Cross Ref]

[22]. Long term effect of reduced pack sizes of paracetamol on poisoning deaths and liver transplant activity in England and Wales: interrupted time series analyses. BMJ 2013; 346 doi: http://dx.doi.org/10.1136/bmj.f403 (Published 07 February 2013) Cite this as: BMJ 2013;346:f403,Keith Hawton, professor of psychiatry and director centre for suicide research1, Helen Bergen, researcher1, Sue Simkin, researcher1, Sue Dodd, scientific assessor2, Phil Pocock, principal statistician3, William Bernal, reader in hepatology4, David Gunnell, professor of epidemiology5, Navneet Kapur, professor of psychiatry and population health6 
[23]. University of Oxford Centre for Suicide Research, Department of Psychiatry, Warneford Hospital, Headington, Oxford OX3 7JX, UK

[24]. Post Authorisation Signal Unit, Vigilance and Risk Management of Medicines, Medicines and Healthcare products Regulatory Agency, London, UK

[25]. Statistics and Clinical Audit, NHS Blood and Transplant, Stoke Gifford, Bristol, UK

[26]. Liver Intensive Therapy Unit, Institute of Liver Studies, King’s College Hospital, London, UK

[27]. University of Bristol, School of Social and Community Medicine, Canynge Hall, Bristol UK

[28]. University of Manchester, Centre for Suicide Prevention, University Place, Manchester, UK 


\title{
Impact of Regulatory Amendment on Human Subjects, Clinical Research Industries and its stakeholder in India.
}

\author{
Article by Kapil Krishna Gupta \\ Ph.D. Clinical Research, Texila American University, India \\ Email: kapil.crcaiims@gmail.com
}

\begin{abstract}
India is one of the major destinations for conducting clinical trials. The Drug Controller General of India (DCGI) is the governing body responsible for all pharmaceutical-research and regulatory issues in India. While conducting clinical trials in India, regulations have come to ensure safety and well-being of the study subjects in the trial.

But, it has seen due to unethical Clinical Trials, Subject's rights were compromised and unprotected. This is one of the main reasons for the clinical research amendment and make regulation subject friendly by protecting the right, safety and well-being of the subject participant in the trial. So, the results of clinical studies are more credible, and authenticate.

But, some of regulatory amendments become a challenge for Research Industries and its stakeholders.

Hence, this study identifies following things:

- Reason of Regulatory amendment in India

- Comparative study between previous and amended regulation

- Impact of this Regulatory amendment on Subject's protection, Research Industry and Stakeholder (includes Sponsors/Investors, Industry, Investigators and Ethic Committee)

\section{Introduction}

In the past decade, India had emerged as a preferred site for drug trials by multinational pharmaceutical companies. India is said to have the largest pool of "naive" patients (i.e. untreated) in many disease areas. Clinical Trials Regulations are basically for to protect the health of the citizen of the country. Its objectives are to prevent mortality in enrolled subjects and also to stop illegal trials.

Against illegal trial, a non-governmental organization filed a public interest lawsuit in February 2012, complaining about unregulated clinical trials of new drugs conducted in India by multinational pharmaceutical companies.

On January 3, the Supreme Court heard the complaint and castigated the Union government "for being negligent in curbing illegal clinical trials despite the deaths of at least 2374 persons who had undergone the dubious testing for unregistered drugs between 2007 and 2012. The Court stated 'The drug trials are creating havoc in the country.'

The Centre had admitted that serious adverse events of deaths during the clinical trials in a period of 2005-2012 were 2644, out of which 80 deaths were found to be attributable to the clinical trials,' the affidavit had said. A parliamentary report estimated that currently 150,000 people are enrolled in clinical trials in India. It estimates that between 2009 and 2012, there were 352,475 people in India enrolled in pharmaceutical tests.

Clinical trial on Human subject to evaluate the safety and efficacy of Investigational drugs, but it must consider the safety and well-being of Human Participant, evaluate benefits and risk ratio before providing to the human subject. But in some case, it was not justified or serious lapses existed. For example, In April 2010 the Indian Council of Medical Research suspended a phase V post-license clinical trial of vaccines against the human papillomavirus amid allegations that the trial had violated rules on informed consent. As many as 25, 000 girls in Andhra Pradesh and Gujarat received the vaccine. The violation 
Texila International Journal of Clinical Research

Volume 3, Issue 2, Dec 2016

came to light when seven girls who received the vaccine died. Subjects were not properly informed about the study.

In India, Drug Controller General of India (DCGI) is the main regulatory authority, which provides approval to conduct clinical trials in India. Along with this Ethics Committee (Institute or Independent) give approval to initiate the clinical study under Institute or Investigator. And the Ethic Committee is having rights to stop a clinical trial in case of misconducting or ask for clarification. But, there is a question mark of the EC as well.

So, Introduced Regulatory Amendment supports all stakeholders to perform their responsibility as per ethical guideline. That helps to protect Human Participant in Clinical Research and make a positive impact on the Clinical Research Industry with credible outcome.

\section{Method and results}

India has a young, highly educated workforce and a large population concentrated in an Urban Centers, high prevalence of all major diseased and knowledge of English makes it easy to set up clinical trial sites in India. India is relatively new to the scene contributing to only about $1 \%$ of all global clinical trials.

As per the revised Schedule ' $Y$ ' of the Drugs \& Cosmetic Act (2005), "a clinical trial is a systematic study of new drug(s) in human subject to generate data for discovering and/or verifying the clinical, pharmacological (including pharmacodynamics and pharmacokinetics), and/or adverse effects with the objective of determining the safety and/or efficacy of the new drugs". Clinical trial of drugs is a randomized, single or double blind controlled study in human participants, designed to evaluate prospectively the safety and effectiveness of new drugs/ new formulations.

The new drug as defined under the Drugs and Cosmetic Rules 1945 (D\&C Rule), and subsequent amendments include:

- A new chemical entity (NCE)

- A drug which has been approved for a certain indication, by a certain route, in a certain dosage regimen, but which is now proposed to be used for another indication, by another route, or in another dosage regimen.

- A combination of two or more drugs which, although approved individually, are proposed to be combined for the first time in a fixed dose combination (FDC).

Drugs Controller General (India) (DCGI) is equivalent to the US Food and Drug Administration (FDA) and European Medicines Agency (EMEA). The DCGI is the official governing body responsible for all pharmaceutical research and regulatory issues in India described in the Drugs and Cosmetics Rules, 2005 (D\&C R). Clinical trials are regulated per Schedule Y of the Drug and Cosmetics Rules.

It has been observed that due to unethical practices, falsified data, noncompliance of GCP guidelines and conducting clinical trials without DCGI approval were some of the existing discrepancies. There are several reports of exploitation of poor, illiterate Indian citizens for clinical trials with increasing reports of trial participant fatalities resulting from the need of strict vigilance and regulations.

Conducting trial in India was easier than in North America or Europe. In India, trial participants were exploited because of illiteracy, poverty and unawareness of basic rights of study participants.

Though, there is a need to promote clinical trials in India, regulations are necessary to ensure safety and well-being of the trial subjects through adequate health insurance and compensations. The Central Drugs Standard Control Organization (CDSCO) has taken a noteworthy step by launching online Clinical Trial Registry -India (CTRI) ensuring accountability, transparency and information sharing on clinical trials in the public domain.

\section{Reason of regulatory amendment in india}

Example of Illegal conduct of Clinical Research: Due to Regulatory uncertainty in clinical research hits drug trials in India. An avalanche of reforms to the rules governing the conduct of clinical trials in India has led to an exodus of drug companies and research organizations. 


\section{Case 1}

- Shirin Abdul Khan was 16 years old when in the year 2009; she was enrolled as a volunteer in a clinical trial to study the effects of adding vitamin D to conventional treatment for patients newly diagnosed with pulmonary tuberculosis. Along with many of her school friends, she was lured by the easy money.

- "It was harmless," Khan says. "We just had to sign a blank form every time the money was given. Whenever I had to go for a check-up, once a month, they would pay us 150 rupees (US\$2.50). It was good money for a school student.”

- She decided to enroll after hearing about her aunt's treatment for cancer. Sairabano Khan was part of a clinical trial in 2008 to study the safety and efficacy of a new compound for the treatment of multiple myeloma.

- Sairabano Khan received 700 rupees (US\$11.00) at home each time she went for a check-up at the Deenanath Mangeshkar Hospital in Pune. This was one month's pay for someone who used to do household chores. She had to stop work because of her illness, and the trial money helped her make ends meet.

There are many such cases in India of money changing hands as volunteers enrolled in trials. However, the companies failed to seek adequate consent from participants in some trials. Sometimes the consent papers were in English, which most patients in rural areas cannot understand, and representatives didn't explain the many risks associated with the trial.

- In 2013, India's Supreme Court took action after receiving complaints and public-interest litigation (PIL) petitions filed by non-government organizations (NGOs). All trials of new drugs were subsequently put on hold. However, the Indian government convinced the Supreme Court that the move was not in the interest of patients who needed new drugs.

- The Supreme Court acceded (approval), but imposed a three-tier screening process for all trials. Since then, the Ministry of Health has laid down fresh rules and amended existing legislation to tighten the regulatory control of clinical trials.

- But uncertainty over the new regulatory regime has led pharmaceutical companies and contract research organizations (CROs) to move their clinical trial program elsewhere.

- Since regulations in India were amended in 2005 in a bid to liberalize the conduct of global drug trials, companies have flocked there because of the genetic diversity of the population. However, trials in the country have been plagued by scandal. Government data show that more than 2,600 patients participating in clinical trials in India died between 2005 and 2012, and nearly 12,000 suffered serious adverse effects. Of these, 80 deaths and more than 500 serious adverse effects were directly attributed to the drug being trialed.

\section{Case 2}

- It was a 2009 US\$3.6m post-licensure observational study, funded by the Bill \& Melinda Gates Foundation, which finally prompted regulatory change.

- The Phase V study (Post-License Clinical Trial), which aimed to evaluate the cost and feasibility of introducing the human papillomavirus (HPV) vaccine into the country's universal immunization program, was run by the Program for Appropriate Technology in Health (PATH), a non-profit organization based in Seattle (United States), the Indian Council of Medical Research (ICMR) in New Delhi, and the Indian state governments of Andhra Pradesh and Gujarat.

- The trial, which involved 24,777 adolescent girls in Andhra Pradesh and Gujrat received the vaccine, was halted by the ICMR in April 2010 following media reports of the deaths of seven participants and a memorandum from '68 human rights and women's groups' send to the Indian Minister of Health and Family Welfare opposing the trial's "unethical” nature. The trial had violated rules of Informed Consent. 
Texila International Journal of Clinical Research

Volume 3, Issue 2, Dec 2016

- "The media came down hard," says Chandra M Gulhati, a healthcare activist in New Delhi and editor of the Indian medical journal Monthly Index of Medical Specialties. "The government had to finally concede defeat."

- The deaths were not found to be causally associated with the vaccine. However, VishwaKatoch, director-general of the ICMR, admitted to India's Parliamentary Standing Committee on Health and Family Welfare in April 2010 that the guidelines laid down by the drug controller general of India (DCGI) had not been adhered to during the trial.

- The DCGI works for the health ministry and is responsible for approving new drugs, clinical trials and medical devices, as well as monitor the quality and efficacy of pharmaceutical products on the market.

- After little action by the Indian Government, the women's health activists who had brought the case to the attention of the Indian Parliament filed a PIL petition in the Supreme Court about unethical promotion of the vaccines in the private and the public sector, violation of rules on informed consent and the need to investigate the deaths and adverse events after vaccination.

- Those required to respond to the petition included the DCGI, the ICMR, the states of Andhra Pradesh and Gujarat, PATH International, and the vaccine manufacturers.

- A second petition on the HPV vaccination project was jointly filed by two NGOs -SAMA, a resource group for women and health, and the Karnataka-based Drug Action Forum - and Delhi Science Forum. These petitions came on the back of earlier PIL petitions on clinical trials filed by NGO Swasthya Adhikar Manch, and doctor and whistleblower Anand Rai.

- In a report published in August 2013, the Parliamentary Standing Committee on Health and Family Welfare said that PATH had violated laws and regulations laid down for clinical trials in India, while conducting the HPV trial, and accused it of promoting the interests of HPV vaccine manufacturers, who would have reaped huge profits had the vaccine been included in the universal immunization program.

- The report was also critical of the ICMR, which forms ethical guidelines for researchers, pointing out that its involvement in the study gave rise to a conflict of interest.

- In a statement, PATH stressed that it strongly disagreed with the findings, conclusions and tone of the report, which it said disregarded the evidence.

\section{Flow chart of case 2}

- June 2009: Registration of clinical trials in the Indian Council of Medical Research (ICMR) registry becomes mandatory.

- 2009: Non-profit organization PATH along with the ICMR and two local governments start a phase 4 trial of human papillomavirus vaccine in adolescents in two Indian states.

- April 2010: 68 Indian human rights and women's groups send a memorandum to the Indian Minister of Health and Family Welfare opposing what they say is the unethical nature of the PATH HPV vaccine trial and calling for it to be halted. In response, the ICMR suspends the trial.

- March 2011: Twelve New Drug Advisory Committees are constituted to evaluate applications for approval of clinical trials, excluding investigational new drugs (INDs). Applications for INDs are evaluated by a separate committee.

- February 2012: Indore and Pune based health activist group, SwasthyaAdhikarManch, files Public Interest Litigation seeking justice for "drug trial victims throughout nation".

- January 2013: Amendments to the Drugs and Cosmetics Rules specify procedures to analyze the reports of serious adverse events occurring during clinical trials and procedures for payment of compensation in case of trial-related injury or death.

- February 2013: Amendments to the Drugs and Cosmetics Rules specify various conditions for conduct of clinical trials, authority for conducting clinical trial inspections and actions in case of 
non-compliance. Further amendments specify requirements and guidelines for mandatory registration of ethics committees.

- March 2013: The Drugs Controller General of India (DCGI) constitutes an expert committee to examine reports of deaths in clinical trials.

- July 2013: The Ranjit Roy Chaudhury panel — established to advise on policy guidelines for approval of new drugs, clinical trials and banning of drugs - publishes a report suggesting major changes, including that clinical trials should be held only at centers that are accredited for the purpose, and that the existing 12 drug advisory committees should be replaced by a single broad expertise-based Technical Review Committee to ensure speedy clearance of applications.

- July 2013: The US National Institutes of Health announces it is suspending 40 clinical trials in India because of the uncertainties posed by the new requirements.

- August 2013: Parliamentary Standing Committee on Health and Family Welfare publishes report criticizing PATH, the Indian Council of Medical Research and the DCGI over conduct of the HPV vaccine trial.

- August 2013: Drugs and Cosmetics (Amendment) Bill 2013 introduced in Parliament, which contains penal provisions for violations of clinical trial procedures, and provisions for payment of compensation and ethics committees.

- August 2013: The DCGI makes it mandatory for the sponsor or his representatives to furnish the details of the contract between the sponsor and the investigator with regard to financial support, fees, honorarium, and payments in kind to be paid to the investigator.

- September 2013: India’s Supreme Court suspends all clinical trials of new drugs in the country.

- September 2013: Contract research organization Quintiles closes its research center in Hyderabad, a joint venture with Apollo Hospitals Enterprise.

- November 2013: The DCGI issues a directive that an audiovisual recording of the process of obtaining written informed consent is required for each trial subject.

- January 2015: The health ministry proposes pre-submission meetings in a bid to enable technical deliberations between stakeholders and the drug regulator before clinical trial applications are submitted.

\section{Other issues}

- Ssues with regard to the ethics committee, Mr. Amar Jasani, a researcher and trainer in the field of bioethics and public health mentioned that "Ethics committees are the front line regulators for clinical trials. If they were functional, they would be a major factor in preventing unethical trials. The problem is the ethics committees are completely controlled by the institutions-they are not at all independent, the people on the committees are not trained, nor do they have the resources or independence to do their job.”

According to Jasani, Indian law allows for commercial ethics committees to be hired by the many CROs they are meant to monitor. "There's a double conflict-of-interest," he said. "They are governed by the CROs or the pharma companies. At the same time they are profit making-so they are more motivated by financial interest rather than the safety of participants."

- Compensation is another contentious issue that is being dealt with in the new directive. Between 2010- 2012, the Drugs Controller General had approved 1,065 clinical trials. Activists say that taking advantage of poverty, illiteracy and lack of awareness, pharmaceutical companies or middlemen, even doctors, often connive to deny compensation to participants when due.

The report clarifies: "Compensation need not be paid for injury or death due to totally proven unrelated causes. In all other cases of death or injury/disability, compensation should be paid to the participant or his legal heirs." The base amount and other calculations are still being worked out. Because of this, the Regulation was also amended in term to provide compensation for participating. 
Texila International Journal of Clinical Research

Volume 3, Issue 2, Dec 2016

- Informed consenting is also an important issue informed consent is a main part of clinical Study by which subject confirms his/her willingness to be a part of clinical studies after having been informed about the various components of the study. But non-compliances also have been seen in this area. For example: "Informed consent from each participant is a mandatory prerequisite for a clinical trial," the CIDSCO report emphasizes. This is an area that unethical practitioners have been taking advantage of, Mishra said "For example, the 16-page directive on this count is translated into local Hindi language that fits into a single page, and that too improperly translated. In Indore, at least 95 percent of participants did not know what it was all about and signed."

So, amended new regulation will provide a frame to properly conduct clinical studies in India. New amendments are being made by government to provide benefits to subject and generate truthful, credible data. It is expected, Regulatory amendment in India will give new and effective rules for subject. Hence, Subject rights can never compromise and subject can get proper informing about inform consent, so he/she can take to participate in trial freely.

\section{Comparative study between previous and amended regulation}

Regulatory Amendment: All amendments to Clinical research, regulation are explained here with their comparative study from pervious to Recent.

\section{1) Regulatory amendment in informed consent process}

Informed consent is a main safeguard for the protection of human subjects in research. It is a process it takes decision of whether or not to participate in a study.

"Informed consent is a process by which a subject voluntarily confirms his or her willingness to participate in a particular trial, after having been informed of all aspects of the trial that are relevant to the subject's decision to participate. Informed consent is documented by means of a written, signed and dated informed consent form."

The two key words in the definition are 'Voluntarily' and 'informed' are the cornerstone of ethical conduct in clinical research and also protect the rights and safety of the trial subjects.

\section{Previous}

- As per schedule Y, in all trials, a freely given, informed, written consent is to be obtained from each study subject. No person can involve in Clinical research as Research Subjects without signed Informed consent.

- The investigator must provide information about the study verbally as well as using a patient information sheet, in a language that is non-technical and understandable by the study subject.

- Informed about all Risk and Benefit ratio, about trial medicine and visits, Subject rights and more as per Protocol and applicable regulatory requirement

- The Subject's consent must be obtained in writing using an 'informed Consent Form'. If the subject or his/her LAR is unable to read/Write - an impartial witness should be present during the entire informed consent process who must append his/her signatures to the consent form.

But, due to non-compliance of this process creates uneventful condition, which make major hazard to subject life or make a serious adverse event.

\section{Example}

A phase V trial, which involved 24,777 adolescent girls in Andhra Pradesh and Gujrat received the vaccine, was halted by the ICMR in April 2010 following media reports of the deaths of seven participants because the trial had violated rules of Informed Consent.

Issue with ICF translation also makes major problem like the 16-page directive on this count is translated into local Hindi language that fits into a single page, and that too improperly translated. In Indore, at least 95 percent of research participants did not know what it was all about and signed. 


\section{ICF PROCESS Amendment in year 2013}

- In the case W.P. (C) No. 33/2012 of SwasthyaAdhikarManch, Indore \& Anr Vs. Ministry of Health and Family Welfare \&Ors. With WP (c) No. 779/2012 regarding clinical trials.

- The Hon’ble Supreme Court, has passed an order dated 21.10.2013.

- As per the said order, in respect of 5 Global Clinical Trials for which approval was given by CDSCO after 01.01.2013 till 31.08.2013, before the clinical trials are conducted, ensures that audiovisual recording of the informed consent process of the Participants is done and the documentation preserver, adhering to the principles of confidentiality.

- In light of the above order of the Hon'ble Supreme Court, CDSCO vide F. No. GCT/20/SC/Clin./2013 DCGI dated 19.11.2013 has issued direction that in all clinical trials, in addition to the requirement of obtaining written informed consent, audio-visual recording of the informed consent process of each trial subject, including the procedure of providing information to the subject and his/her understanding in such consent is required to be done while adhering to the principle of confidentiality.

- Such audio-visual recording and related documentation would be preserved. This is applicable to the new subjects to be enrolled in all clinical trials including Global clinical trials.

\section{ICF PROCESS Amendment in year 2015}

As per Drug \& Cosmetics rules, Notification Dated 31 Jul2015 of the Government of India in the Ministry of Health and Family welfare (Department of Health and Family Welfare), number G.S.R. 364 (E) the Gazette of India, Extraordinary, Part II, section 3, sub-section (i). The rules may be called the Drugs and Cosmetics (Fifth Amendment) Rules, 2015. Amendment in the Drugs and Cosmetics Rules, 1945, in Schedule Y-

In paragraph 2 under the heading "Clinical Trial”, in sub-paragraph (4) relating to "Informed Consent”, after clause (iii), the following shall be inserted, namely:-

"(iv). An audio-video recording of the informed consent process in the case of vulnerable subjects in clinical trials of New Chemical Entity or New Molecular Entity, including procedure of providing information to the subject and his understanding on such consent, shall be maintained by the investigator for record.

Provided that in case of clinical trial of anti-HIV and anti-Leprosy drugs, only audio recording of the informed consent process of individual subject including the procedure of providing information to the subject and his understanding on such consent shall be maintained by the investigator for record.”

\section{2) Regulatory amendment in 'essential elements' of informed consent}

\section{Previous:}

a. Essential Elements

1) Statement that the study involves research and explanation of the purpose of the research

2) Expected duration of the Subject's participation

3) Description of the procedures to be followed, including all invasive procedure

4) Description of any reasonably foreseeable risks or discomforts to the Subject

5) Description of any benefits to the Subject or others reasonably expected from research. If no benefit is expected Subject should be made aware of this

6) Disclosure of specific appropriate alternative procedures or therapies available to the Subjects

7) Statement describing the extent to which confidentiality of records identifying the subject will be maintained and who will have access to Subject's medical records

8) Trial treatment schedules (s) and the probability for random assignment to each treatment (for randomized trials)

9) Compensation and /or treatment (s) available to the Subject in the event of a trial-related injury 
Texila International Journal of Clinical Research

Volume 3, Issue 2, Dec 2016

10) An explanation about whom to contact for trial related queries, rights of Subjects and in the event of any injury

11) The anticipated prorated payment, in any, to the Subject for participating in the trial

12) Subject's responsibilities on participation in the trial

13) Statement that participation is voluntary, that the subject can withdraw from the study at any time and that refusal to participate will not involve any penalty or loss of benefits to which is Subject is otherwise entitled

14) Any other pertinent information

\section{Amendment in 'Essential Element' of informed consent in year 2013}

Amendment in Scheduled Y (Drug and Cosmetic Act) dated Nov'2013; in Appendix V, Under the heading "INFORMED CONSENT", and in sub-heading 1.1 relating to "Essential Elements", for serial number 9 amended as follow:

"9. Statement describing the financial compensation and medical management as under:

a. In the event of an injury occurring to the clinical trial subject, such subject shall be provided free medical management as long as required.

b. In the event of a trial related injury or death, the Sponsor or his representative, whosoever has obtained permission from the licensing Authority for conduct of the clinical trial, shall provide financial compensation for the injury or death.”

\section{Amendment in 'Essential Element' of informed consent in year 2015}

In APPENDIX V, under the heading 'INFORMED CONSENT' in sub-heading 1.1 relating to

'Essential Elements', following serial number shall be substituted, namely:

“9. Statement describing the financial compensation and medical management as under:

a. In case of an injury occurring to the subject during the clinical trial, free medical management shall be given as long as required or till such time it is established that the injury is not related to the clinical trial, whichever is earlier.

14. Statement that there is a possibility of failure of investigational product to provide intended therapeutic effect.

15. Statement that in the case of placebo controlled trial, the placebo administered to the subjects shall not have any therapeutic effect.

16. Any other pertinent information.”

\section{3) Regulatory amendment in serious adverse event reporting and timeframe}

Previous SAE and timeframe

- Serious Adverse Event is any untoward medical occurrence that at any dose :

- Result of death

- Is life-threatening

- Requires inpatient hospitalization or prolongation of existing hospitalization

- Result in persistent or significant disability/incapacity

- is a congenital anomaly/birth defect

- Investigator report SAE to Sponsor within 24 hour and to Ethic committee within 7 Working days of SAE Occurrence.

- Sponsor must send report of SAE within 14 Calendar days to DCGI and other participating Investigators of SAE Occurrence.

\section{Amendment SAE and timeframe in year 2013}

- The Drugs \& Cosmetics Rules have been amended vide GSR no 53 (E) dated 30-01-2013 inserting a Rule 122DAB, and a new Appendix-XII in Schedule Y along with other amendments. 
- The amendments specifies the detail procedures for analysis of Serious Adverse Events (SAEs) including deaths occurring during clinical trial to arrive at the cause of death / injury to the subject, as the case may be, and to determine the quantum of compensation, if any to be paid by the sponsor or his representative whosoever have obtained permission from CDSCO in a time bound manner.

- As per the provisions, each SAE including death is required to be examined and decision regarding causality of death and quantum of compensation, if any, is required to be taken by CDSCO in a time bound manner as per the procedure specified in Appendix XII of Schedule Y.

- As per Appendix XII the Investigator shall report all serious and unexpected adverse events to the CDSCO, the Sponsor or his representative whosoever had obtained permission from the CDSCO for conduct of the clinical trial and the Ethics Committee, within twenty four hours of their occurrence.

- In case of serious adverse events of death, the reports shall be examined by an independent Expert Committee constituted by DCG(I) to determine if the cause of death is due to following reasons, which are considered as clinical trial related death and gives its recommendation to CDSCO. In case of clinical trial related death the committee shall also recommend the quantum of compensation to be paid by the sponsor or his representative, to CDSCO.

1) Adverse effect of investigational product(s);

2) Violation of the approved protocol, scientific misconduct or negligence by the Sponsor or his representative or the investigator;

3) Failure of investigational product to provide intended therapeutic effect;

4) Use of placebo in a placebo-controlled trial;

5) Adverse effects due to concomitant medication excluding standard care, necessitated as part of approved protocol;

6) For injury to a child in-utero because of the participation of parent in clinical trial;

7) Any clinical trial procedures involved in the study

- CDSCO shall consider the recommendations of the Expert Committee and shall determine the cause of death and also the quantum of compensation in case of clinical trial related death within three months of receiving the report of SAE of death.

In cases of serious adverse event other than death, CDSCO shall determine the cause of injury, if any, due to any of the reasons mentioned above as in the case of death, which is considered as clinical trial related injury. However CDSCO has option to constitute an independent Expert Committee, wherever considered necessary, to examine such serious adverse event. In case of clinical trial related injury, CDSCO shall also determine the quantum of compensation within three months of receiving of the SAE.

- In case of clinical trial related injury or death, the Sponsor or his representative concerned shall pay the compensation as per the order of CDSCO within thirty days of the receipt of such order.

- In order to streamline the submission of reports of SAEs, a system of pre-screening of reports of SAEs at the time of receiving these reports is being introduced in CDSCO.

\section{The pre-screening system will be as under}

- The preliminary scrutiny of the SAE reports will be done by CDSCO officer(s) based on laid down checklist which is attached herewith. During the preliminary examination, the CDSCO officer(s) will scrutinize the SAE reports to ensure that it contains all the required administrative as well as technical information in proper manner as per the checklist. If SAE reports are not submitted in accordance with the format and the checklist, it will not be accepted by CDSCO for further examination.

- Once a report of SAE is accepted, the information in the report will be reviewed by CDSCO as per the specified procedures. 
Texila International Journal of Clinical Research

Volume 3, Issue 2, Dec 2016

- The sponsor or his representative conducting clinical trials in India are requested to prepare the SAE reports for submission to CDSCO as per appendix-XI of Schedule-Y of D\&C Rules and the checklist enclosed.

- The SAE reports must be submitted with proper binding, indexing and page number. Without indexing of page number, no SAE report will be accepted.

(a) The reports of SAEs of deaths should be prepared and submitted in red cover.

(b) The reports of SAE of injury other than deaths should be prepared and submitted in blue cover.

(c) The SAE report other than that mentioned at (a) \& (b) above is to be prepared and submitted in white cover.

- Clear and unequivocal information should be provided in the SAE report.

- Text and tables should be prepared using margins that allow the document to be printed clearly without losing any information and the left-hand margin should be sufficiently large so that information is not obscured by the method of binding. The documents printed on both sides of a page, can be submitted. However, one should take care that the information is not obscured when the page is placed in a binder.

- While submitting reply to a query, the applicant should always enclose with the reply, a copy of query letter issued by CDSCO.

- All items mentioned in the checklist may not be applicable in all the case of SAE ${ }^{e e}$. The items not relevant to a particular SAE should be marked with "Not Applicable (NA)".

This system of preliminary screening to determine the acceptability of the SAE report will come into effect from 18.02.2013

\section{Amendment SAE and timeframe in year 2014}

- As per Notification of Ministry of Health and Family welfare (Department of Health and Family welfare) dated $12^{\text {th }}$ Dec, 2014. GSR 889(E), whereas a certain rules further to amend the Drugs and Cosmetic Rules, 1945 was published. These rules may be called the Drugs and Cosmetics (Sixth Amendment) Rules, 2014.

In the drugs and cosmetics Rules, 1945-

In rule 122DAB-

- For sub-rule (1), the following sub-rule shall be substituted, namely:-

"(1) In case of an injury occurring to the subject during the clinical trial, free medical management shall be given as long as required or till such time it is established that the injury is not related to the clinical trial, whichever is earlier."

- After sub-rule (2), the following sub-rule shall be inserted, namely:-

"(2A) In case, there is no permanent injury, the quantum of compensation shall be commensurate with the nature of the non-permanent injury and loss of wages of the subject.”

- In sub-rule (5)

In clause (c), after the words "therapeutic effect", the words, "where, the standard care, though available, was not provided to the subject as per the clinical trial protocol" shall be inserted;

In clause (d), after the words, "Placebo controlled trial", the words, "where, the standard care, though available, was not provided to the subject as per the clinical trial protocol” shall be inserted;

\section{In schedule $\mathbf{Y}$}

- In paragraph 2 relating to 'CLINICAL TRIAL',

In sub-paragraph (2), relating to 'Responsibilities of Sponsor', for clause (iv), the following clause shall be substituted, namely:-

"(iv) Any report of the serious adverse event, after due analysis shall be forwarded by the sponsor the Licensing Authority as referred to in Clause (b) of rule 21, the Chairman of the Ethics Committee and the head of the institution where the trial has been conducted, within fourteen days of the occurrence of the serious adverse event." 
- In sub-paragraph (3), relating to 'Responsibilities of the Investigator(s)', in clause (i), for the portion beginning with the words "The report of the serious adverse event of death" and ending with the words " occurrence of the serious adverse event.", the following shall be substituted, namely:-

"In case, the investigator fails to report any serious adverse event within the stipulated period, he shall have to furnish the reason for the delay to the satisfaction of the Licensing Authority along with the report of the serious adverse event. The report of the serious adverse event, after due analysis, shall be forwarded by the Investigator to the Licensing Authority as referred to in cause (b) of rule 21, the chairman of the Ethics Committee and the Head of the Institution where the trial has been conducted within fourteen days of the occurrence of the serious adverse event.”

- In sub-paragraph (5), relating to 'Responsibilities of the Ethics Committee', for clause (iv), the following clause shall be substituted, namely:-

"(iv) In case of serious adverse event occurring to the clinical trial subject, the Ethics Committee shall forward its report on the serious adverse event, after due analysis, along with its opinion on the financial compensation, if any, to be paid by the Sponsor or his representative, whosoever had obtained permission from the Licensing Authority as referred to thein clause (b) of rule 21 for conducting the clinical trial, to the Licensing Authority within Thirty days of the occurrence of the serious adverse event."

- In sub-paragraph 5(A), relating to 'Serious Adverse Events', in clause (2).-

The Words "and unexpected" shall be omitted;

After the words "and pass orders as deemed necessary", the following shall be inserted, namely:-

"In case, the investigator fails to report any serious adverse event within the stipulated period, he shall have to furnish the reason for the delay to the satisfaction of the Licensing Authority along with the report of the serious adverse event.”

In Appendix V, in serial number 1, in item number 1.1, in sub-item number 9, for clause (a), the following clause shall be substituted, namely:-

"(a) in case of an injury occurring to the subject during the clinical trial, free medical management shall be given as long as required or till such time it is established that the injury is not related to the clinical trial, whichever is earlier.”

In Appendix XII, For serial number (1), the following shall be substituted, namely

"(1) In case of an injury occurring to the subject during the clinical trial, free medical management shall be given as long as required or till such time it is established that the injury is not related to the clinical trial whichever is earlier."

- In serial number (2) after the words, "medical management of the subject”, the words, "In case, there is no permanent injury, the quantum of compensation shall be commensurate with the nature of the non-permanent injury and loss of wages" shall be inserted;

- In serial number (5)-

In clause (d), after the words, "therapeutic effect", the words, "where, the standard care, though available, was not provided to the subject as per the clinical trial protocol" shall be inserted;

In clause (e), after the words 'placebo-controlled trial', the words, "where, the standard care, though available, was not provided to the subject as per the clinical trial protocol” shall be inserted;

- In serial number (6),-

In clause (a),

The words, "and unexpected" shall be omitted

After the words "occurrence as per Appendix XI.”, the following shall be inserted, namely:-

"In case, the investigator fails to report any serious adverse event within the stipulated period, he shall have to furnish the reason for the delay to the satisfaction of the Licensing Authority along with the report of the serious adverse event.”

- In clause (b), in Para (i). 
Texila International Journal of Clinical Research

Volume 3, Issue 2, Dec 2016

- In sub-para (B)-

The words, "chairman of the Expert Committee with a copy of the report to", shall be omitted;

For the words "ten calendar days", the word "fourteen days" shall be substituted;

- In sub-paragraph (C)-

The words "to the Chairman of the Expert Committee with a copy of the report", shall be omitted;

For the words "twenty-one calendar days", the words "thirty days shall be substituted;

After the sub-paragraph (C), the following shall be inserted, namely:-

"(CA) The Licensing Authority shall forward the report of the Investigator, Sponsor or his representative whosesoever had obtained permission from the Licensing Authority for conducting clinical trial and the Ethics Committee to the Chairman of the Expert Committee."

- In sub-paragraph (D), for the words "thirty days of receiving the reports from the Ethics Committee" the words, "one hundred and five days of the occurrence of the adverse event" shall be substituted;

- In sub-paragraph (G), for the words "three months of receiving the report of the serious adverse event", the words, "one hundred and fifty days of the occurrence of the adverse event" shall be substituted;

- In para number (ii),-

- In sub-paragraph (A), for the word "ten calendar days", the words, "fourteen days" shall be substituted;

- In sub-paragraph (B), for the words "twenty one calendar days", the words, "thirty days" shall be substituted;

- In sub-paragraph (D), for the words, "three months of receiving the report of the serious adverse event", the words, one hundred and fifty days of the occurrence of the adverse event shall be substituted.

\section{4) Regulatory amendment of EC registration in feb'2013}

- Drug and Cosmetic Rule amendment vide GSR no. 72 (E) dated 08-Feb-2013 inserting Rule 122DD, in Schedule Y along with other amendments. The amendment specifics the detail procedure for the registration of Ethic Committee.

- As per Rule 122 DD, No Ethic Committee shall review and accord its approval to Clinical Trial Protocol without prior Registration with DCG (I). An application for registration of the Ethic Committee shall be made to the DCG (I) in accordance with the requirement as specified in the Appendix VIII of Schedule Y.

- In order to streamline the submission of application for registration of Ethics Committee and their examination as per Rule 122DD, it has been decided to introduce a system of preliminary scrutiny of such applications at the time of their receipt, to determine their acceptability for examination by CDSCO.

\section{5) Number of clinical studies conduct by investigator jul'2014}

- As per DCGI recommendation, the sponsor shall ensure that the number of Clinical Trial an investigator can undertake should be commensurate with the natures of the trial, facility available with the Investigator. However, under no circumstances the number of trials should be more than three at a time. Effective Date 03-Jul-2014

- Re-Amendment Aug'2016: Removal of Restriction of conducting 3 clinical trials per Investigator. Effective date 02 Aug 2016 
Brief chart of clinical research amendment in form year 2013 to Nov' 2016

\begin{tabular}{|c|c|c|}
\hline Document Name & File Name/ Order \# & $\begin{array}{l}\text { Effective } \\
\text { date }\end{array}$ \\
\hline $\begin{array}{l}\text { Removal of Restriction of conducting } 3 \text { clinical trials per } \\
\text { Investigator. Effective date } 02 \text { Aug } 2016\end{array}$ & $\begin{array}{l}\text { File No.12-01/14- } \\
\text { DC(Pt.47) }\end{array}$ & 2-Aug-16 \\
\hline $\begin{array}{l}\text { Removal of condition for the requirement of } 50 \text { Bedded } \\
\text { site from clinical Trial. Effective date } 02 \text { Aug } 2016\end{array}$ & $\begin{array}{l}\text { File No.12-01/14- } \\
\text { DC(Pt.47) }\end{array}$ & 2-Aug-16 \\
\hline $\begin{array}{l}\text { Requirement of NOC form DCGI for addition of new } \\
\text { clinical trial site or investigator. Effective date } 03 \text { Aug } \\
2016\end{array}$ & $\begin{array}{l}\text { File No.12-01/14- } \\
\text { DC(Pt.47) }\end{array}$ & 3-Aug-16 \\
\hline A-V Consenting in case of Vulnerable subjects for ICF & GSR 611(E) & 31-Jul-15 \\
\hline SAE Compensation and SAE reporting time line & GSR. $889(\mathrm{E})$ & 12-Dec-14 \\
\hline Providing Ancillary care for CT Subject & $\begin{array}{l}\text { File No.12-01/14- } \\
\text { DC(Pt.47) }\end{array}$ & 3-Jul-14 \\
\hline Clinical Trial on Medical Device & $\begin{array}{l}\text { File No.12-01/14- } \\
\text { DC(Pt.47) }\end{array}$ & 3-Jul-14 \\
\hline $\begin{array}{l}\text { Requirement for Filing of application to market new } \\
\text { Chemical Entities }\end{array}$ & $\begin{array}{l}\text { File No.12-01/14- } \\
\text { DC(Pt.47) }\end{array}$ & 3-Jul-14 \\
\hline Limited number of clinical trial an Investigator & $\begin{array}{l}\text { File No.12-01/14- } \\
\text { DC(Pt.47) }\end{array}$ & 3-Jul-14 \\
\hline CT-Compensation in case of Injury or Death & $\begin{array}{l}\text { File No.12-01/14- } \\
\text { DC(Pt.47) }\end{array}$ & 3-Jul-14 \\
\hline A-V consenting of each trial Subject & $\begin{array}{l}\text { GCT/20/SC/Clin./20 } \\
13\end{array}$ & $\begin{array}{l}19-N o v- \\
13\end{array}$ \\
\hline EC Registration & G.S.R 72(E) & 8-Feb-13 \\
\hline Submission of Report of SAEs to CDSCO & GSR no $53(\mathrm{E})$ & 30-Jan-13 \\
\hline
\end{tabular}

Impact of regulatory amendment on subject's protection/ clinical research industry/ stake holders

\section{Evaluation the result of regulatory amendment}

For evaluating the impact of the regulatory amendment on Human Subject, Research Industry and Stakeholders; I preferred to ask questions from personnel who are active parts of the clinical research process and conduct clinical trials like: Investors, different department in Clinical Research Organization (Operation, Regulatory Record, Data Management, Safety and others) and Investigators. With these feedbacks, Result of Regulations Amendment can identify. Here, I will explain the impact of the amendment with feedback. 
Texila International Journal of Clinical Research

Volume 3, Issue 2, Dec 2016

\section{1) Serious adverse event report and timeframe}

- SAE Compensation, reporting and system of pre-screening for submission of report toCDSCO [In 30JAN2013, File no. 12-01/13-DC (Pt.13-A)]

- Inserting a rule 122DAB and new Appendix-XII in Schedule ' $\mathrm{Y}$ '

- The amendment specifies the detail process for analysis of SAEs include deaths and Injury during clinical trial and determine the quantum of compensation to be paid by Sponsor and his representative

- In this amendment, CDSCO has Changed all stakeholder timelines and structure of reporting SAEs

- In case of Death, the report examined by the Independent Expert Committee (constituted by DCGI) determines the cause of death and this committee also recommends the quantum of compensation to be paid by the sponsor and his representative.

- CDSCO has constituted an independent Expert committee in case of death and injury, shall determine the quantum of compensation within three months of receiving of the SAE.

- In case of Clinical trial related injury or death, the sponsor or his representative shall pay the compensation within 30 days of receipt of such order.

- This regulation also provides a checklist by which CDSCO officer can scrutiny of SAE reports.

- Feedback: Overall feedback of this regulation was Negative due to following reason:

- Quantum of compensation will decide by the Independent Expert Committee, no any guideline was available for calculation of quantum of compensation. Sponsor and his representative need to follow Expert committee decision.

- Due to undefined expanse, this regulation impacting negative, as this is something extra expenses will come over the sponsor.

- Reporting to Independent expert committee, involving Unexpected SAE, provide less time in reporting also giving Negative impact of this regulation.

- $\quad$ SAE Compensation and reporting timeline [In 12Dec2014, File GSR.889(E)]:

- With consider the above response, CDSCO make amendment in D\&C act, after doing consultation with the Drugs Technical Advisory Board.

- In rule 122DAB- (1) In case of any injury occurring to the subject during the clinical trial, free medical management shall be given as long as required or till such time it is established that the injury is not related to the clinical trial, whichever is earlier.

- After Sub-rule (2), '2A-In case, there is no permanent injury, the quantum of compensation shall be commensurate with the nature of the non-permanent injury and loss of wages of the subject.

- Direct sending SAE report to Expert Committee has omitted.

- Timeline of reporting has modified/increased for all stakeholders after due analysis.

For Sponsor, report sent duration increase from 10 Calendar days to 14 days to License Authority, EC and Head of Institute where trial has conducted.

For Investigator, Unexpected adverse event and direct report to Expert Committee has omitted. After a due analysis report duration has changed from 10 days to 14 days.

For EC, After due analysis SAE reporting timeline has changed to 21 days to 30 Calendar days.

Feedback: Points which were negative, now it's become Positive after this Amendment.

- Positive response received as Unexpected SAE has omitted, Time frame increase for due analysis, direct reporting to Expect committee has omitted.

- The response received, the report can evaluate properly and correct report forwarded to License Authority and other stakeholder.

- On 03JUL2014, Guideline "CT-Compensation in case of Injury or Death" also introduced by CDSCO. This guideline is available for calculating Quantum of compensation. So, Investor and other stakeholder can calculate it. The unpredictable cost issue has also been resolved with this. 


\section{2) Informed consent process of subjects}

- A-V consenting of each subject of each trial Subject [In19Nov2013, File number GCT/20/SC/Clin./2013]:

- In case W.P. (c) No. 33/2012 of SwasthyaadhikarManch, Indore \&Anr Vs. Ministry of Health and Gamily welfare \&Ors., the Hon'ble Supreme Court, has passed an order dated 21.10.2013. Before the clinical trials are conducted, Audio-Visual recording of the informed consent process of the Participants should perform of each subject.

- Add Essential elements in Informed consent Form, hence subject gets informed about detail description and requirement of Clinical Trial.

- Storage of A-V consent at least a period of 5 years if it is not possible to maintain the same permanently.

Feedback: Received Negative feedback about this amendment.

- Most of the subjects were not ready for A-V consenting, as subject feeling uncomfortable with this process.

- As received feedback from Investigator and Research Coordinator, Subject's start feeling afraid and makes negative perception about clinical research, before any explanation about research.

- In leprosy or HIV trial or any major critical illness, it becomes difficult to take an audio-video consenting with Subject and his/her legal representative, as they don't want such consenting due to embarrass feeling.

- Due to this expected number of subjects in Clinical trial was not matched, putting negative impression of this amendment and affect the growth of Clinical Market as well.

- Due to this regulation, many investigators don't want to involve and process of Informed consent process due to undefined fear factors.

- Audio-Visual consenting is a time consuming and lengthy procedure.

- For this site need specific set-up where they can seat and defined or described all point to subject

- Storage of consent was also an issue for some investigator or Clinical Coordinator in some place.

- A-V Consenting in case of vulnerable subjects[In 31Jul2015, GSR 611(E)]:

- These rules called the Drug and Cosmetic (Fifth Amendment) Rules, 2015.

- In paragraph 2 under heading 'Clinical Trial', in sub-paragraph (4) relating to 'Informed Consent', after clause (iii)-

(iv) An audio - video recording of the informed consent process in the case of vulnerable subjects in clinical trials of New Chemical Entity or New Molecular Entity including procedure of providing information to the subject and his understanding on such consent, shall be maintained by the investigator for the record.

- Provided that in case of a clinical trial of anti-HIV and anti-Leprosy drugs, only audio recording of the informed consent process of individual subjects, including the procedure of providing information on the subject and his understanding on such consent shall be maintained by the investigator for record.

- Modified and add in Sch. Y, Appendix V, under heading 'Informed Consent'-

14) Statement that there is a possibility of failure of Investigational product to provide intended therapeutic effect

15) Statement that in the case of placebo controlled trial, the placebo administered to the subjects shall not have any therapeutic effect.

Feedback: Received Positive feedback

- A-V consenting to each subject (on 19Nov'2013), provide a Negative impact on stakeholder of this amendment, but this negative impression turns to Positive with amendment of A-V Consenting in case of Vulnerable Subjects for ICF. 
Texila International Journal of Clinical Research

Volume 3, Issue 2, Dec 2016

- Now, with this amendment, Subject feels comfortable to be a part of Clinical study. Anti-HIV and Anti-Leprosy also not feeling embarrassed to be a part of the study, as they go with the audio consenting process in case of New Chemical Entity or New Molecular entity.

3) Ethic committee registration [In 08Feb2013, file number GSR 72 (E)]

- Insert rule $122 \mathrm{DD}$, Registration of Ethics committee with the requirements as specified in Appendix VIII of Schedule Y.

- So, No Ethic Committee shall review and accord its approval of a clinical trial protocol without prior registration with the Licensing authority.

- In case of SAE occurring to the subjects during clinical trial, the EC shall analyze and forward its opinion as per procedure specified in Appendix XII of Sch. Y

- Registration valid for a period of Three years from the date of Issue, so Ethic Committee will apply for re-registration by License Authority before expiry.

- Feedback: Received Positive feedback about this amendment.

- Registration of the EC by CDSCO, gives positive impact among all stakeholders.

- It's helpful to conduct research under surveillance and protect trial subject's rights, safety as well.

- Now, EC actively involved in site activities and issues. Like-

- As per CDSCO regulation on 02Aug2016, Removal of Restriction of conducting 3 clinical trials per Investigator; it is further decided that an Ethic Committee after examining the risk and complexity involved in the trial being conducted/proposed shall decide about how many trials an investigator can undertake.

- As per CDSCO regulation on 02Aug2016, Removal of condition for the requirement of $\mathbf{5 0}$ Bedded sites from clinical Trial; regard to this amendment, No clinical trial shall be conducted on site having less than 50 bedded hospitals, it has been decided to revise this condition and it is further decided that Ethics Committee shall examine and decide whether the clinical trial site is suitable for trial or not irrespective of number of beds.

So, as per received feedback, overall impact about the Clinical Research Regulatory Amendment is positive and Effective in term of:

- Conduct clinical research with proper regulation become mandatory

- Protecting the rights, safety and well-being of Trials Subjects become more strong and effective

- During Informed consent process, Subject rights to know all risk and benefit of study and subject is free to take decision to participate in the study.

- Protection of study subjects are more secured due to effective reporting of SAE and Compensation guideline

- Site, including Investigator and Coordinator must perform their responsibility as per regulation

- Ethic committee become more responsible to regulate clinical trial on site in positive term, that help to take decision and action against any discrepancy on site level

- All regulations are well instructed and written, that make clear understanding and view for investors to conduct clinical research in India.

- With all effective regulation, investors become aware and secure to get effective, credible and authenticated clinical data and good protection of human participant in a Clinical Trial.

- It is expected as per received feedback and response, these regulations help to improve:

- Subject safety during clinical trial

- Increase interest of Stakeholder toward India and ultimately it will give growth to Clinical research market in the near future in India.

\section{Conclusion}

The updated guidance on conducting clinical trials in India is steadily improving, as DCGI is committed to amend and upgrade the policies to encourage research in India. This emerging trend in clinical research indicates broad based effectiveness oriented approach. 
The road was bumpy and there were lots of hurdles. The recent regulatory amendments give a fresh breath of air to the Clinical Research industry, which was gripped by ethical issues and non-transparency. Most of the issues have resolved in the past few years and also successful to cover the loopholes in the regulations and make India a trial and patient friendly global destination.

In recent years, Indian regulatory authorities have been quite aggressively working toward all fronts, with the goal of enhancing the quality and integrity of research through optimization of approval timelines. The stringent process for both regulatory approval and patient recruitment had a severe impact on the clinical research industry.

The Indian clinical research industry booms up, with implementation of all regulatory amendments made by CDSCO. It will make a drastic change or growth in the Indian clinical research market in near future.

\section{Reference}

[1]. http://www.cdsco.nic.in/forms/list.aspx?lid=1585\&Id=1

[2]. Sama: Resource Group for Women and Health. Memorandum to the Health Minister on World Health Day opposing HPV vaccinations. April 2010.

[3]. Department of Health Research, Ministry of Health and Family Welfare. Seventy-second report on alleged irregularities in the conduct of studies using Human Papillomavirus (HPV) vaccine by PATH in India. August 2013. [4]. Report of Professor Ranjit Roy Chaudhury expert committee to formulate policy and guidelines for approval of new drugs, clinical trials and banning of drugs. July 2013.

[5]. Pandey A, Aggarwal AR, Seth SD, Maulik M, Bano R, Juneja A. Clinical trials registry_-India: redefining the conduct of clinical trials. Indian J Cancer2008;45:79-82.

[6]. Mudur G. Human papillomavirus vaccine project stirs controversy in India. BMJ2010;340:c1775.

[7]. AHRP/cms/content/view/898/84/ (Internet reference about Mr. Amar Jasani statement)

[8]. THE HEALTH SITE/news/no-more-clinical-trials-till-you-can-monitor-them-supreme-court-to-centre/

[9]. JANANEETHI/jananeethi/projectregard/papernews/supremecourt_onclinicaltrial_4-1-12.pdf

[10]. IPSNEWS/2011/10/india-unauthorised-clinical-trials-on-bhopal-victims/

[11]. Dhananjay Mahapatra.2644 died during clinical trails during 7 years, The Times of India, Oct, 2012.

[12]. Hob’ble Mr. Justic R.M. Lodha, Hon’ble Mr. Justic Anil R. Dave. SwasthayaAdhikaManch, Indore \& Anr. Vs.

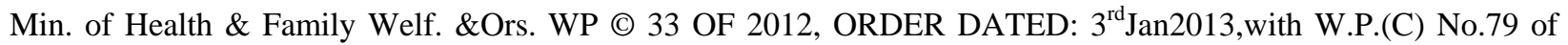
2012, http://courtnic.nic.in/supermecourt/temp/wc\%203312p.txt,8.10.2012,26.7.13

[13]. Ethical Guidelines for Biomedical Research on Human Participants Indian Council of Medical Research, New Delhi, 2006

[14]. The Drug and Cosmetic (Amendment) Bill, 2013 (Bill No. LVIII OF 2013)

[15]. The Indian Medical Council (Professional conduct, Ethic \& Etiquettes) Regulation, 2002, 7.22 CHAPTER 7 : MISCONDUCT

[16]. Minutes of the meeting of the Ethics Committee held on 20th November, 2012 at 09:00 A.M. in the Council Office, Sector-8 pocket-14, Dwarka, New Delhi-77. Available at: hhtp:www.mciindia.org/meetings/Ethics/2012/Minutes_20.11.2012.pdf

[17]. Pawan Kumar Bansal. Politicians who exposed malpractices in medical sector. Available at: http:www.merines.com/article/plioticians-who-exposeed-mal[ractices-inmedicalsector/15894017.shtml\#sthash.ctMh8LEV.Fhc3l1z.dpuf

[18]. 'www.unethicalclinicaltrial.org/Case_studies.aspx’' of swasthyaadhikarmanch 


\title{
Adverse Drug Reaction Reporting Bv Doctors in a Developing Country: a Case Study from Ghana
}

\author{
Article by Mark Korankye \\ M.Sc Clinical Research, Texila American University, Ghana \\ Email: marklister85@gmail.com
}

\begin{abstract}
This paper reviewed an original article written by Sabblah et al on the adverse drug reaction reporting by medical doctors in a region in Ghana in 2012 but article originally published in 2014.

All over the world, spontaneous adverse drug reaction reporting is the mostly used method of monitoring the safety of drugs. This method is challenged with underreporting by healthcare professionals. The original article seeks to assess the knowledge of doctors about the adverse drug reaction (ADR) reporting system in Ghana, the rate of reportage and the attitude of the doctors on spontaneous reporting of ADRs. The method used was a cross-sectional survey and a selfadministered questionnaire was used to collect data.

This review was to summarize and critique the content of the study and state possible recommendations. In critiquing, the authority, accuracy, currency and objectivity of the study were assessed. The study's relevance, stability and graphs/tables were also assessed. In the article there were no recommendations or limitation to the study itself, recommendations were however made for improving spontaneous reporting. The results cannot be generalized so further studies are recommended in a national scope.
\end{abstract}

Keywords: Ghana; spontaneous reporting; ADR; doctors; review; underreporting

\section{Source}

Sabblah GT, Akweongo P, Darko D, Dodoo AN, Sulley AM. (2014) Adverse drug reaction reporting by doctors in a developing country: a case study from Ghana: Ghana Medical Journal, December, Vol. 48, no. 4, pp189-193. Doi: 10.4314/gmj.v48i4.4. Accessed on 5th September 2016 at http://www.ajol.info/index.php/gmj/article/view/112883

\section{Introduction}

The article "Adverse drug reaction reporting by doctors in a developing country: a case study from Ghana" by Sabblah George et al. seeks to assess the knowledge of doctors about the adverse drug reaction (ADR) reporting system in Ghana and the rate of reportage. The study also sought to assess the attitude of the doctors on spontaneous reporting of ADRs.

This article was published in journal for doctors managed by Ghana Medical Association. It is the right journal for the impact needed for the ADR reporting advocacy. Spontaneous ADR reporting is a basic mechanism for pharmacovigilance and so reportage from doctors is quite significant. A reportage of 6 per one million population per year, and out of which only $12 \%$ is contributed by doctors really needs an investigation. This is so, especially when no work has been done to study the rate of reporting ADRs by healthcare professionals in Ghana. Under-reporting is the biggest challenge of spontaneous reporting of ADRs and any study to ascertain the causes and possible resolution to such hindrances is a study in the right direction and purpose of good pharmacovigilance practice.

In this paper, attempt will be made to summarize the article at hand and assay whether the methods or structure of the study and article achieved its aim. This review will also scrutinize the accuracy, currency, authority and the objectivity of the claims made per the information gathered against the prevailing situation. Relevant changes or happenings in the area of ADR reportage since the article 
Texila International Journal of Clinical Research

Volume 3, Issue 2, Dec 2016

publication would be highlighted, and suggest areas where additional research findings would help the understanding of reporting of ADRS.

\section{Review of literature}

ADR reporting is a significant public health issue and of a concern to the pharmaceutical industries and their regulators. Spontaneous reporting of ADRs is a fundamental tool widely used to the success of pharmacovigilance activities all over the world (1). The WHO Program for International Drug Monitoring (PIDM) sets the optimal rate of ADR reporting at over 200 reports per million inhabitants per year (2). Some developed countries in Europe like UK, Sweden, Australia, France and Denmark fall within the range of 300-500 per year $(3,4)$ whiles developing countries such as Ghana has as low as 6.

From this recorded reportage, the healthcare team is expected to contribute the majority. Whiles some countries have quite an open and easy system of reporting where the general public or patients could report, it is not so in less developed countries. The contribution of the healthcare professionals is hugely significant, and cannot be overemphasized (5). In countries where the spontaneous reports has been low, like Africa, the expectation is that the healthcare team would adequately lead the campaign and act accordingly but this has not been the case. Though no work has been done yet in Ghana for a thorough rate of reporting by healthcare professionals, some studies in Nigeria have suggested records of between $2-32 \%$ of the spontaneous reports in that country to be from health professionals $(6,7)$.

The reality of the case therefore suggests that there is generally under-reporting of ADRs and the healthcare professionals are the group from which measures must be addressed to correct this appalling situation. Inman (8) in his popular study has described some of the factors, mostly attitudinal, contributing to such under-reporting of ADRs where he captures them as the "seven deadly sins". These "sins" could be grouped into those bothering on the profession (rewards and financial incentives, litigations and ambition to publish cases) and, knowledge and attitude (complacency, ignorance, indifference and diffidence).

The above literature has been significantly referred to because of their empirical impact on the study conducted in the article.

\section{Article summary}

The primary essence of the study was to measure the frequency with time of reporting adverse drug reaction by medical doctors as part of the health professionals in Greater Accra region of Ghana. Certain factors and attitudes influence the responsibility of the doctors in reporting ADRs, the article sought to identify some these factors and attitudes.

The study suggests that prior training of a medical doctor in reporting would influence the tendency to report a case of adverse event. And it was shown in the study that majority of the doctors were not trained in ADR reportage as against only $27 \%$ trained ones, who were from the government or quasigovernment hospitals. A doctor's participation in clinical research or the number of patient seen per day did not have significant influence on reporting. However, the longer number of years of practice than as a house officer is likely to influence spontaneous reporting.

Almost all (96\%) all of the doctors agreed that they were the most responsible professional to report ADR, lack of time, the commonness or unserious nature of the ADR and the non-availability or inaccessibility of reporting forms could not make them report even with the least chance of having an individual case.

Training and refresher courses be embarked on by the Pharmacovigilance Centre, and report forms be made available and accessible to the doctors. Acknowledgment of receipt and feedback on submitted report could improve the rate of reporting. 


\section{Article structure}

The article was introduced with a summary which comprised of a background to the topic revealing the aim of the study and the need for the research. The method used for the collection of data and the type was also described including the type of statistics or data analysis; descriptive, frequencies and percentages. The results of the research was captured briefly in the summary mostly stating the status of the outcome measures in percentages, then the conclusion of the article was abridged and simplified into the summary.

The main body of the article was arranged under 5 headings; the introduction, methods, results, discussion and conclusion. The introduction was carefully arranged under four-paragraphed thematic areas the history of the subject in literature, the challenge(s) of the subject, the situation in other continents and neighboring countries and the possible causes. The objectives of the study was added to conclude the introduction section.

The methods of the study were described in details under subheadings ranging from the study design and location, sampling, data collection and quality control, data processing and analysis and ethical considerations. This is a well-structured format for revealing the methodology for the study. The results from the data processing and analysis was comprehensibly laid out in three well-arranged tables for easy inferencing and a figure to display the reasons for reporting ADRs.

The discussion of the results of the study was on point in few paragraphs though covered all the measuring outcomes. It covered the impact of the background and knowledge of the doctors on ADR report. The discussion also covered whether the type of hospital facility and the experience of the doctors had impact on the likelihood to report. The conclusion was however very short but fairly on point. The references followed which were chronological arranged in the Modern Language Association format.

\section{Article critique}

\section{Authority}

The Ghana Medical Journal is a quarterly peer-reviewed journal for the Ghana Medical Association but opened to all aspects of health with readership including students, researchers, other health care professionals, policy makers, pharmaceutical representatives, press and the public. The Lancet is a partner and the texts are also available online at African Journal Online and PubMed Central.

The corresponding author is a researcher with the Food and Drugs Authority in Ghana in the Safety Monitoring and Clinical trials Division. He has this and other articles cited by other publications on similar subjects and the contributing authors are known researchers especially Dodoo A.N., so credibility of the authors is thus established.

\section{Accuracy}

The publication of the article was based on a current research work its position is supported by the other current research information and literature review in the article. The article was also published in a peer-reviewed journal which contributed in the accuracy of the article. The references used in the article were fairly current, accurate and well related to the subject of the study.

\section{Currency}

The data for the research work was collected in 2012 and the article published in 2014 and so could be described as current. The year of publication of the reference used ranges from 1998 to 2011. Per the prevailing conditions of the practices spontaneous reporting, not so much has changed from the articles results. The article generally reflects the reality of the current situation in Ghana.

\section{Relevance}

This article's aims are targeted at a real challenge of pharmacovigilance and to some extent in public health. Under-reporting of ADRs and the apathy of doctors regarding spontaneous reporting of 
Texila International Journal of Clinical Research

Volume 3, Issue 2, Dec 2016

ADRs challenges the monitoring of drugs in Ghana. It is therefore relevant to have the content of the article to educative the doctors of what the reality of ADR reporting. Individually the doctor may know the situation as it pertains to their facility but such a study has painted a bigger picture of it.

The content of the article is also relevant to other health care professionals, regulators, policy makers (health ministry), and heads of health institutions as well as the general public. Through this study, the trainings on spontaneous reporting could be intensified and possibly influence other decisions such as empowering other health professionals to report ADRs even from the time of professional training. The article is therefore also relevant for the academicians, and health practice and clinical researchers.

\section{Objectivity}

The article was well written and the data objectively collected based on current practices regarding the subject. Doctors were selected randomly involving the government and private practitioners, specialists to house officers and both genders. The methods employed and the tools for the processing of the data as well as its analysis contributed to the objectivity. Fairly there was minimal introduction of bias though the study could have increased the scope of the sample from just one capital region to others farther from the south.

Per the kind of data collected, the author objectively treated and analyzed them and discussed the results without being bias. Relevant references quoted by the article and the similarities in the outcome measures also supported this claim of the article being objective. The authors however did not state any limitation to the study conducted in their article.

Limitations like the simple random selection of the participant could affect the results of the survey. The claim by the doctors who reported ADR could not be verified or compared with the reality per the data from the National Pharmacovigilance Centre and this is a limitation which can affect the results. The questionnaires could have been sent via mail and the responds sent likewise so that more time could have been used to respond instead of the rush in responding, but it is understandable if the purpose was to improve responds rate.

\section{Stability}

The Ghana Medical Journal, African Journal Online and PubMed are authentic and reliable journals whose contents can be deemed stable. The stability is further entrenched by the articles data collection being based on practice oriented settings.

\section{Analysis of graph/tables}

The article had three different tables and a graph covering outcome measures from background of the participating doctors, their training in spontaneous reporting, the Odds ratios of ADR reporting and their reasons for not reporting ADRs seen.

There were thrice as much doctors below the age 40years than those above. More than half of the respondents were Medical Officers (52.5\%) with the rest being specialist and house officers, and $50.2 \%$ were from teaching hospital level and again 50\% saw between 25-44 patients per day. Approximately $66 \%$ had less than 8years practice experience and $77 \%$ working for the government.

The quasi-government facilities had more doctors among them trained in ADR reporting (approx. $40 \%$ ) than their government counterparts (26\%), with the private sector doctors reporting as low as $11 \%$. The most significant factors for the participating doctors not reporting seen ADRs are nonavailability of the reporting forms and ignorance of the reporting procedures.

\section{Recent advances related to the topic}

Searching through online journals in PubMed for "spontaneous reporting of ADR" or "adverse drug reaction reporting", many article were sited with similar conclusions of lack of knowledge or ignorance of procedures for spontaneous reporting by various health professionals worldwide except for well developed countries. 
Akshaya et al. (2016) through a systemic review suggested that pharmacovigilance activities in India was low and as huge as $75 \%$ of had never reported any ADR and that there was a big polarity in terms of knowledge, attitude and practice against pharmacovigilance activities (9).

According to Yun Mi Yu et al. (2016), the rate of spontaneous report by community pharmacist in Korea was as high as $95 \%$ and this could be attributed to the continuous education and training of health professionals about ADR reporting and their experiences with ADR cases. According to the article, these professionals do not allow or were less concerned about the obstacles to reporting (10). Similarly in Malesia, other health professions aside doctor and pharmacist, like nurses, were assigned more responsible roles of reporting ADRs especially those pertaining to vaccines. This contributed to a steep rise in ADR reports with respect to children (11). There are other initiatives by some countries to increase rates of spontaneous reporting.

In Turkey, there has been a gradual increase in reporting rates due to the increased awareness of pharmacovigilance activities and introduction of the practice of having a pharmacovigilance contact points (PvCPs, a physician/pharmacist) in the various hospital facilities to champion the course of ADR reporting.

Other initiatives like the establishment of pharmacovigilance inspections in the health facilities and the empowerment of the patients to also report directly to the pharmacovigilance centers which is already available in certain European countries like Denmark, Sweden, Australia and Netherlands (12, 13). In relation to the involvement of the public in reporting is the initiative of incorporating technological tools to the usual reporting forms. This will be in the interest of both the public and the health care professional alike with the use of texting-based ADR reporting system or smart phones and through other form of internet communications $(14,15)$.

The pharmacovigilance activities in Africa is still in its development and there more non-member state to the international monitoring program to join this all important program. There are more interventional measures to be undertaken to correct the under-reporting of ADRs by just studying the above sited literature (16).

\section{Conclusion}

The article by Sabblah et al. "Adverse drug reaction reporting by doctors in a developing country: a case study from Ghana”, generally was well written, arranged and results objectively discussed. This review was to summarize and critique the content of the study and state possible recommendations. In the article there were no recommendations or limitation to the study itself, recommendations were however made for improving spontaneous reporting. The results cannot be generalized so further studies are recommended in a national scope. There are new national campaigns where patients are encouraged to report ADR especially to community pharmacies, so it will be interesting to test the impact and the rate of reporting from the pharmacist perspective. This review has to some extent added some value to scientific literature by the constructive critique of a study on ADR spontaneous report.

\section{References}

[1.] Aagaard L, Stenver DI, Hansen EH. (2008) Structures and processes in spontaneous ADR reporting systems: a comparative study of Australia and Denmark. Pharm. World Sci. 30(5):563-570. Doi: 10.1007/s11096-008-9210-y https://www.ncbi.nlm.nih.gov/pubmed/18350372

[2.] Ahmad SR. (2003) Adverse drug event monitoring at the Food and Drug Administration. J Gen Intern Med. 285:437-443 https://www.ncbi.nlm.nih.gov/pmc/articles/PMC1494803/

[3.] Akshaya Srikanth Bhagavathula, et al. (2016) Health Professionals' Knowledge, Attitudes and Practices about Pharmacovigilance in India: A Systematic Review and Meta-Analysis. PLoS One, 11(3): e0152221. doi:10.1371/journal.pone.0152221 https://www.ncbi.nlm.nih.gov/pmc/articles/PMC4807086/

[4.] Blenkinsopp A, Wilkie P, Wang M, Routledge PA. (2007) Patient reporting of suspected adverse drug reactions: a review of published literature and international experience. Br J ClinPharmacolFeb;63(2):148-156. 
Texila International Journal of Clinical Research

Volume 3, Issue 2, Dec 2016

DOI:

10.1111/j.1365-2125.2006.02746.

http://onlinelibrary.wiley.com/doi/10.1111/j.1365-

2125.2006.02746.x/full

[5.] Belton KJ. (1997) Attitude survey of adverse drug reaction reporting by health care professionals across the European Union. The European Pharmacovigilance Research Group. Eur. J. Clin. Pharmacol 52(6):423-7. https://www.ncbi.nlm.nih.gov/pubmed/9342576

[6.] Bello SO, Umar MT.(2011) Knowledge and attitudes of physicians relating to reporting of adverse drug reactions in Sokoto, north-western Nigeria. Ann Afr Med 10(1): 13-18. Available from: http://www.annalsafrmed.org/article.asp?issn=1596-

3519; year=2011; volume=10;issue $=1$; spage $=13$; epage $=18$; aulast $=$ Bello

[7.] Enwere OO, Fawole OI. (2008) Adverse drug reactions reporting by physicians in Ibadan, Nigeria. Pharmacoepidemiology and Drug Saf. 17:517-522. https://www.ncbi.nlm.nih.gov/pubmed/18404621/

[8.] GulnihalOzcan, (2016) Adverse Drug Reaction Reporting Pattern in Turkey: Analysis of the National Database in the Context of the First Pharmacovigilance Legislation. Drugs Real World Outcomes, Mar; 3(1): 33-43.doi: 10.1007/s40801-015-0054-1 https://www.ncbi.nlm.nih.gov/pmc/articles/PMC4819489/

[9.] Haggar H. Ampadu et al. (2016) Adverse Drug Reaction Reporting in Africa and a Comparison of Individual Case Safety Report Characteristics Between Africa and the Rest of the World: Analyses of Spontaneous Reports in VigiBase ${ }^{\circledR}$. Drug Saf, 39: 335-345. doi: 10.1007/s40264-015-0387-4 https://www.ncbi.nlm.nih.gov/pmc/articles/PMC4796322/

[10.] Inman WH. (1996) Attitudes to adverse drug-reaction reporting. $\mathrm{Br} J$ ClinPharmacol 41:433-5. https://www.ncbi.nlm.nih.gov/pubmed/8735689/

[11.] JoëlleBerrewaerts et al. (2016) Patient Participation and the Use of Ehealth Tools for Pharmacovigilance.

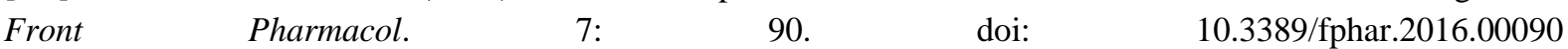
https://www.ncbi.nlm.nih.gov/pmc/articles/PMC4827142/

[12.] Pal S et al. (2011) The world medicines situation 2011: Pharmacovigilance and safety of medicines. Geneva: WHO, 2011 [cited 2016, 23 September]. Available from: http://apps.who.int/medicinedocs/documents/s18771en/s18771en.pdf

[13.] RoslianaRosli (2016) A Retrospective Analysis of Spontaneous Adverse Drug Reactions Reports Relating to Paediatric Patients. PLoS One, 11(6): e0155385. doi: 10.1371/journal.pone.0155385 https://www.ncbi.nlm.nih.gov/pmc/articles/PMC4889073/

[14.] The WHO Pharmacovigilance Reporting Trends. [Cited 2016, September 2016].Available from: http://www.whoumc.org/DynPage.aspx?id=108476\&mn1=7347\&mn2=7252\&mn3=7322\&mn4=7558

[15.] Vergeire-Dalmacion G, Castillo-Carandang NT, Juban NR, Amarillo ML, Tagle MP, Baja ES (2015) Texting-Based Reporting of Adverse Drug Reactions to Ensure Patient Safety: A Feasibility Study. JMIR Public Health Surveill, $\quad$ Jul-Dec; $\quad 1(2): \quad$ e12 $\quad$ DOI: $10.2196 /$ publichealth.4605 https://www.ncbi.nlm.nih.gov/pmc/articles/PMC4869238/

[16.] Yun Mi Yu et al. (2016) Predictive Factors of Spontaneous Reporting of Adverse Drug Reactions among Community Pharmacists. PLoS One, 11(5): e0155517. doi: 10.1371/journal.pone.0155517 https://www.ncbi.nlm.nih.gov/pmc/articles/PMC4871451/ 


\title{
An overview of the importance of the Trial Master File (TMF) and the required contents in clinical trials as stipulated in the ICH GCP guidelines
}

\author{
Article by Catharina Susanna Venter \\ M.Sc in Clinical Research, Texila American University, South Africa \\ Email: carinventer19@gmail.com
}

\begin{abstract}
Aim: The Trial master File (TMF) is a method used worldwide to collect and file documents related to clinical trials. The aim of this study was to review the ICH GCP guidelines related to the TMF and to provide an overview of the requirements of the TMF.

Method: A review of the ICH CGP guidelines related to the TMF and additional resources.

Topics reviewed: Six main topics was identified and discussed to provide an overview: What is a Trial Master File (TMF), the importance and benefits of the TMF, TMF and essential documents as outlined in the ICH GCP E6 guidelines from section 8.1 to 8.4, electronic TMF (eTMF), additional documents filed in the TMF

Conclusion: The TMF set-up and maintenance during a clinical trial is essential to ensure all the required documents are filed and to ensure all the relevant documents are available and audit ready at all times.
\end{abstract}

Keywords: Trial Master File, TMF, GCP, essential documents, investigator, sponsor

\section{Introduction}

The validity and integrity of a clinical trial is essential to obtain approval from the ethics committee and regulatory bodies to conduct clinical trials in compliance with International Conference of Harmonization (ICH) Good Clinical Practice (GCP) guidelines. The Trial Master File (TMF) is a compilation of these essential documents obtained during initiation, conduction and close out of clinical trials. These documents stored in the TMF are important to prove the validity of the clinical trial, indicate the quality of the data obtained and provides prove of ICH GCP compliance for all the personnel involved in the clinical trial. The ICH CGP guidelines provides as list of all the essential documents needed to be filed in the TMF and their purpose in the clinical trial.

A procedure or plan should be in place to make sure the documents in the TMF is comprehensive and correct and should be able to provide all the necessary information to reconstruct the clinical trial from beginning to end precisely.

\section{Method}

The TMF plays an important role in clinical trials and consists of a collection of essential documents obtained during the conduct of the trial. For this investigation, the ICH E6 guidelines on the TMF are reviewed with the support of additional resources to identify the role of the TMF in a clinical trial and various concepts related to the TMF.

\section{Literature overview}

\section{What is a trial master file (TMF)}

Documents collected before, during and after a clinical trial provides prove that the study was conducted, the data collected is correct and valid and that the investigator and sponsor conducted the trial according to ICH GCP guidelines. These documents are collected at the site and by the sponsor and are collectively known as the TMF. The TMF consists of various essential documents as required by the different regulatory authorities. The TMF is started before the start of a clinical trial, 
Texila International Journal of Clinical Research

Volume 3, Issue 2, Dec 2016

maintained through the conduct of the trial and after the trial to ensure all the required documents are collected until close out of the trial. ${ }^{1,2}$

\section{The importance and benefits of the TMF}

Documents required for the TMF is important for several reasons related to the overall validity and integrity of the study. The collection and filing of essential document in the TMF is not only needed for new product approval but can be beneficial as well. ${ }^{1,2,3}$

Importance:

a) Evaluation of the validity and quality of the data collected

b) Required as prove the trial was conducted in accordance with the ICH CGP guidelines and other specified regulatory requirements

c) Evidence that both the investigator and sponsor was GCP compliant during the conduct of the study

d) Provides crucial information regarding the specific clinical trial to auditor's during an audit

e) The trial can only be completed upon collection of the last document and after it has been reviewed by the monitor to confirm all the required documents are present

Benefits:

a) The clinical trial can be reconstructed through the documents collected

b) The TMF provides an central access point to all the important documents for all the personnel involved in the conduct of the trial

c) The TMF is set-up in a chronological order ensuring easy access to the documents to auditor's and trial personnel

d) Timely collection of these documents contributes to the successful management of a clinical trial

\section{TMF and essential documents as outlined in the ICH GCP E6 guidelines from section}

\section{1 to 8.4}

As per the ICH GCP E6 guidelines section 8.1 essential document are defined as ${ }^{1}$ :

Essential Documents are those documents that individually and documents that individually and collectively permit evaluation of the collectively permit evaluation of the conduct of a trial and the quality of the conduct of a trial and the quality of the data produced. These documents serve data produced. These documents serve to demonstrate the compliance of the investigator, sponsor, and monitor with investigator, sponsor, and monitor with the standard of GCP and with all the standard of GCP and with all applicable regulatory requirements.

As per the ICH GCP E6 guidelines from section 8.1 to 8.4 a list has been compiled outlining the minimum essential documents that has to be collected during the conduct of a clinical trial. Specific documents are collected at different stages of the trial as specified in the ICH GCP E6 guidelines section 8.2 to $8.4^{1}$ :

1. Before the start of clinical phase of the trial

2. During the execution of the trial

3. After termination or completion of the trial

The TMF is set-up right at the beginning of the trial at both the sponsor's office and the investigator's/institution's site. The documents as indicated in Appendix 1 should be audit ready and available for an audit and inspections by the regulatory authorities at any given time. ${ }^{1}$

\section{Electronic TMF (eTMF)}

Due to the increased complexity of studies, especially oncology studies, and the difficulty managing paper TMF's for different departments, most organisations have moved over to eTMFs. ${ }^{2}$

The eTMF consists of various folders to account for all the different documents retained in a trial. The eTMF includes the essential documents, documents specific to the different departments including Laboratory, Biostatistics, Medical Monitor, Clinical Trial Supplies, IRB/EC, Data 
Management, agreements and contracts and several more documents to ensure all the required documents for the different department are present. Unblinded areas are also set-up to ensure there is no premature unblinding and the integrity of the data stays intact. ${ }^{2}$

Different section may also be set-up in the TMF to ensure central or core documents are filed in one section and all site related section is filed in their respective sections as a method of access control to ensure confidentiality is not breached, especially during the conduct of an audit. ${ }^{2}$

The files and documents in the TMF should be filed in a chronological order as predefined in a type of folder structure or predefined directories and it is advised to keep this unified across studies instead of creating new folder structures or predefined directories for each trial. This will allow for flexible and general folders and naming conventions throughout and sections in the TMF that will not be used for a specific trial can be removed or indicated as not applicable. ${ }^{2}$

The use of an eTMF in clinical trials has various advantages such as detailed audit trials that have proven to be very useful in audits and inspections and changes to the documents can be tracked in the audit trial to ensure compliance with the applicable documents and regulations. ${ }^{2}$

\section{Additional documents filed in the TMF}

Apart from the essential documents, it is also necessary to ensure other study related documents are filed. $^{2}$

All previous versions of documents should also be kept in the TMF to ensure in order to be able to keep track of changes made in the documents during the conduct of the study. These documents include protocols, informed consent forms, electronic case report forms, and investigator's brochure. ${ }^{2}$

Important correspondence should also be retained. Important decisions between the sponsor and site, sponsor and CRO, site/sponsor and regulatory authorities and any other communication that contains critical trial related information should be filed in the TMF. These documents range from letters to electronic emails. ${ }^{2}$

\section{Quality control and access control of the TMF}

Additionally to the essential and other required documents that should be filed in the TMF, a system should also be in place to ensure controlled access to the TMF and quality control should be performed to ensure the filed documents are legible and valid.

Access control requires provision and revoking of access through password protected user accounts as needed, restrictions on the type of access depending on the individual role of the user and an audit trial indication all the required information to accurately track uploads and changes to documents in the TMF. ${ }^{2}$

Additionally each document should undergo a quality control process to ensure the documents are legible, accurate and valid. These quality controls are predefined by the sponsor, site and CRO and every document owner should be compliant through the conduct of the study. Quality controls or quality checks include ensuring documents are filed in the same format, all required signatures are present, scanned documents are legible and not information has been removed or cut off in the process and all page numbers are present. Quality control is not just limited to the identification of issues and deficiencies, but also required corrective and preventative actions to eliminate and reduce future risk for the same quality issues. ${ }^{3,4}$

\section{Conclusion}

There are many important aspects to consider with regards to the TMF and this process many be complicated and time consuming depending on the complexity of the study. The TMF should always be audit ready, in a chronological order, complete and accurate and portray conduct of a study as a whole to ensure the study is valid and to confirm the integrity of the data.

Although the TMF has improve tremendously over the past years and moved over to and electronic system allowing better control and audit trials, there is still room for improvement. As studies evolve to be more specific, complex and target specific, the TMF should also be developed to accommodate 
Texila International Journal of Clinical Research

Volume 3, Issue 2, Dec 2016

for these changes to ensure all required documents are collected in the TMF and access to the TMF is restricted and controlled at all times. Future development of the TMF should focus on better access control, more user friendly systems, built in quality control checks and allow for easier identification of missing or incomplete documents.

\section{References}

[1] ICH Guidelines. (1997). Federal Register, 62(90), 25691-25709.

[2] European Medicines Agency. Reflection paper on GCP compliance in relation to trial master files (paper and/or electronic) for management, audit and inspection of clinical trials. London: EMA; 2015. [Cited 2016 Nov 06]. Available from:

http://www.ema.europa.eu/docs/en_GB/document_library/Scientific_guideline/2013/02/WC500138893.pdf. [3] Mielebacher, J. (2015). Volume 7 Issue 422 Journals for Clinical Studies Electronic Trial Master File: Gaining Efficiency for Oversight and Control. JCS, 7(4).

[4] Pereira P. Quality in clinical research activities: Role of institution/clinical trial site. J Nat Accrued Board Hosp Healthcare Providers [serial online] 2015 [cited 2016 Nov 6]; 2:4-8. Available from: http://www.nabh.ind.in/text.asp?2015/2/1/4/160232 


\title{
Adherence to antiretroviral therapy among people living with HIV and AIDS in Monze and Nyimba districts of Zambia
}

\author{
Emmanuel Mwila Musenge ${ }^{1}$, Reuben Kaonga ${ }^{2}$, Dorothy Phiri Sitali ${ }^{3}$, Jessy Zimba ${ }^{4}$ \\ ${ }^{1}$ Ph.D. Clinical Research, Texila American University, Zambia \\ ${ }^{2,3,4}$ Department of Nursing Sciences, School of Medicine, University of Zambia, Lusaka, \\ Zambia \\ E-mail: ${ }^{1}$ emmanuel.musenge@unza.zm, ${ }^{2}$ reubenkaonga@gmail.com, \\ 3chibalekawaz@gmail.com, ${ }^{4}$ jessyzimba@gmail.com
}

\begin{abstract}
Background: The control of human immunodeficiency virus and acquired immune deficiency syndrome depends on several factors which include life-long commitment to antiretroviral therapy. Poor adherence to antiretroviral therapy leads to serious consequences such as drug resistance and eventually high morbidity and mortality. We assessed adherence to antiretroviral therapy and associated factors among people living with human Immunodeficiency virus and acquired immune deficiency syndrome.

Materials and methods: This cross-sectional study was conducted in Monze and Nyimba districts of Zambia. A simple random sample of 364 consenting participants attending antiretroviral therapy clinic was selected between February and March 2015. A structured interview schedule was used to collect data. Multivariate binary logistic regression analysis was carried out using IBM ${ }^{\circledR} S P S S^{\circledR}$ for windows version 20.0 to ascertain the factors associated with adherence to antiretroviral therapy among the participants.

Results: Of the 364 participants, $83.5 \%$ had high adherence to antiretroviral therapy while $16.5 \%$ had low adherence. Knowledge of antiretroviral therapy (OR 0.27, 95\% CI: $0.14-0.54$ ) and antiretroviral therapy clinic site (OR 2.34, CI: 1.25 - 4.40) were statistically significantly associated with adherence to antiretroviral therapy in our study.

Conclusion: Most of the participants were adherent to antiretroviral therapy. There is still need to give more information on human immunodeficiency virus and acquired immune deficiency syndrome and adherence to people living with human immunodeficiency virus and acquired immune deficiency syndrome especially before the initiation of antiretroviral therapy. Also, health care providers need to establish other possible barriers to antiretroviral therapy adherence so as to manage the patients better.
\end{abstract}

Keywords: Human Immunodeficiency Virus, Acquired Immune Deficiency Syndrome, Adherence, Antiretroviral Therapy

\section{Background}

The human immunodeficiency virus (HIV) and acquired immune deficiency syndrome (AIDS) crisis is the worst the world has ever experienced. There were approximately 36.9 million people living with HIV and AIDS (PLWHA) at the end of 2014 with 2.0 million people becoming newly infected with HIV in 2014 globally [1].

The vast majority of PLWHA are in low- and middle-income countries. The sub-Saharan Africa to which Zambia belongs is the most affected region, with about 25.8 million PLWHA in 2014 and accounts for almost $70 \%$ of the global total of new HIV infections [1]. The majority of the HIV infected people are adults between 15 and 49 years. The significant higher proportions of the infected are women [1]. In Zambia, 13\% of adults age 15-49 are infected with HIV (15\% of women and 11\% of men) with an estimated 30,000 deaths [2]. 
Texila International Journal of Clinical Research

Volume 3, Issue 2, Dec 2016

The HIV continues to be a major global public health issue, having claimed more than 34 million lives so far. In 2014, about 1.2 million people died from HIV-related causes globally [1]. The HIV epidemic not only affects the health of individuals, it impacts households, communities, and the development and economic growth of nations. Many of the countries hardest hit by HIV also suffer from other infectious diseases, food insecurity, and other serious problems [3].

There is no cure for HIV infection. However, effective treatment with antiretroviral (ARV) drugs can control the virus so that people with HIV can enjoy healthy and productive lives and reduce the risk of transmitting the virus to others [1]. Thus, the WHO and Joint United Nations Programme on HIV and AIDS (UNAIDS) launched the "3 by 5" program to provide quality care with ART to 3 million PLWHA by the end of 2005 worldwide [4]. Regionally, some African countries have put interventions to promote adherence such as poverty reduction, fight illiteracy, building of more infrastructure and communication to promote accessibility to ART, sensitization and education campaigns on adherence due to cultural and religious beliefs so rife in most African countries [5].

There has been therefore, free ART provision to promote adherence due to social, financial and economic constraints [6]. The number of PLWHA on ART in resource-poor countries has dramatically increased in the past decade. In 2014, 14.9 million PLWHA were receiving ART globally, of which 13.5 million were receiving ART in low- and middle-income countries. The 14.9 million people on ART represent about 40\% of PLWHA globally [1].

Zambia started free antiretroviral (ARV) drugs provision in public health institutions in 2004 to help clients who were unable to sustain the cost of ARV drugs [7]. Zambia has rapidly scaled up ART provision and rolled out even in hard to reach areas through mobile ART activities as one of the recent initiatives to enhance adherence to ART [8]. There are approximately 75,000 PLWHA on ART and only represents about $63 \%$ of people in Zambia who need them [9].

Although provision of ART has been a major stride in the mitigation of HIV and AIDS in Zambia, adherence to ART needs more attention. Increased funding for ART in developing countries has brought to the attention the special emphasis on adherence to all those on ART [10].

In recent years, the WHO has reported that, adherence among patients suffering from HIV and AIDS averages 70\% [11]. Several studies have been conducted among PLWHA in developed and developing countries to ascertain adherence. In the United States of America (USA), adherence rates of $<80 \%$ were reported and nonadherence was related to substance and alcohol abuse [12]. Kim et al. [13] reported that the adolescents and young adults population were adherent to ART. The lowest average ART adherence was in North America (53\%), Europe (62\%), and South America (63\%) and, with higher levels in Africa (84\%) and Asia (84\%). In India, Achappa at al. [14] reported that 63.7\% of the participants were adherent to ART and financial constraints, forgetting to take medication, lack of family care, depression, alcohol use, social stigma and side effects to ART were barriers for adherence.

Ketema and Weret [15], in Ethiopia revealed a 95.5\% adherence among the study participants and having emotional or practical support positively encouraged ART adherence. Also, Reda and Biadgilign [16], against expectations, reported that sub-Saharan Africa patients have similar or higher adherence levels compared to those of developed countries. The challenges to ART adherence included factors related to patients and their families, socioeconomic factors, medication, and healthcare systems.

In Zambia, Katongo [17], Nsakanya [18] and Birbeck [19] reported 82\%, 79.5\% and 60\% adherence among PLWHA on ART respectively. The adherence was related to stigma, information, education and communication, change in daily routine, social support and marital status.

The shift to the use of Highly Active Anti-retroviral Therapy (HAART) for treating HIV infection has led to increasingly complex drug regimens. This treatment presents significant challenges to both patients and health-care providers with respect to adherence. The poor adherence to ART, however, is common in all groups of treated individuals and thus client's adherence problems need to be effectively addressed [20]. 
We assessed adherence to ART and associated factors among PLWHA in Monze and Nyimba districts of Zambia. The findings of this study will contribute to improvement in adherence to ART and reduction in treatment failure leading to good treatment outcome of the patients.

\section{Materials and methods}

\section{Design and sampling procedures}

This cross-sectional study was carried out at Monze Mission and Nyimba District Hospitals among PLWHA. Monze Mission Hospital is a second level Hospital located in Southern Province 300 kilometres from Lusaka while Nyimba District Hospital is a first level Hospital located in Eastern Province 400 kilometres from Lusaka, the capital city of Zambia [21]. The patients attend the ART clinics at appointed times advised by the health care providers for continuous monitoring and consultation about their disease.

All the PLWHA on ART for at least six months and aged 18 years and above were include in the study. The participants who agreed to participate in the study were asked to give informed and written consent. However, the newly enrolled PLWHA for ART and those who were recruited in the previous month(s) were excluded from the study. A simple random sampling method was used and participants were selected consecutively from February to March 2013 to avoid sampling bias. The participants were selected based on the daily sampling frame. The sample size of 364 participants was calculated based on the prevalence formula.

\section{Data Collection}

A structured interview schedule was used to collect data on socio-demographic characteristics, adherence and associated factors. The same tool was used on all the patients to ensure reliability and validity. The data on socio-demographic characteristics, adherence and associated factors were obtained by interview and review of medical records of the patients.

\section{Analyses}

Statistical analyses were carried out using IBM ${ }^{\circledR}$ SPSS $^{\circledR}$ Statistics for Windows Version 20.0 (IBM Corp. Armonk, NY, USA). The frequencies and descriptive statistics of the variables were calculated. The Chi-squared and Fisher's exact tests were used to select potential predictors of high or low adherence to ART. The Odds Ratio and 95\% confidence interval were calculated using multivariate binary logistic regression to identify predictors of adherence to ART while adjusting for confounders. A $p$-value of $<0.05$ was considered significant.

\section{Ethics}

This study was approved by the University of Zambia, School of Medicine Undergraduate Research Ethics Committee (Assurance No. FWA00000338, IRB00001131of IOR G0000774).

\section{Results}

\section{Participants' distribution and characteristics}

Of the 364 participants comprising the study, 216 (59.3\%) were females and 172 (47.3\%) were aged between 26 and 35 years. Most (229; 62.9\%) of the participants were married and $222(61.0 \%)$ were Christians. The majority (286; 73.6\%) of the participants never or had primary education. Fewer than half $(176 ; 48.4 \%)$ of the participant were formally employed and more than half $(200 ; 54.9 \%)$ attended ART clinic at Nyimba District Hospital. Most (304; 83.5\%) of the participants had high adherence while only a few $(60 ; 16.5 \%)$ had low adherence to ART.

The majority (289; 79.4\%) and (316; 86.8\%) of the participants lived more than $15 \mathrm{~km}$ from the ART clinic and reported waiting time of more than 60 minutes respectively. The income of fewer than half (159; 43.7\%) of the participants was moderate (ZMW 1,100 - 1,600) and most (312; 85.7\%) of the participants had adequate knowledge about HIV and AIDS and ART adherence. Fairly over half 
Texila International Journal of Clinical Research

Volume 3, Issue 2, Dec 2016

(209; 57.4\%) of the participants reported good staff attitude to PLWHA. Most (318; 87.4\%) of the participants had good health status and the majority (332; 91.2\%) of the participants had good attitude towards their situation.

\section{Factors associated with adherence to ART}

The Chi-square and Fisher's exact tests were run to select the variables to include into the binary logistic regression model. The distribution of adherence to ART among the participants by the sociodemographic and adherence factors are shown in Tables 1 and 2. There was an association between adherence to ART and knowledge, staff attitude, and ART site. However, age, sex, marital status, religion, education, employment, monthly income, distance, waiting time, health status, and client attitude, were not associated with adherence to ART (Tables 1 and 2).

The multivariate binary logistic regression model was tested for multicollinearity, Hosmer and Lemeshow test of model fitness for data, omnibus test of model coefficients and classification accuracy. The dependent variable was adherence to ART: High (1), Low (0). The results of the multivariate binary logistic regression analysis to predict whether three variable factors, namely staff attitude, knowledge, and ART site were associated with adherence to ART revealed that only knowledge was statistically significantly associated with adherence to ART (Table 3).

The patients who had adequate knowledge on HIV and AIDS and ART were 73\% (OR 0.27, 95\% CI: $0.14-0.54$ ) less likely to achieve high adherence to ART compared to those who had inadequate knowledge. In addition, the patients who visit Nyimba ART site were 2.34 times (OR 2.34, 95\% CI: 1.24 - 4.49) more likely to achieve high adherence compared to those who visit Monze ART site (Table 3).

\section{Discussion}

In the past years, the global fund (GF) emerged to fight HIV infection and to finance the scaling up of resources to promote adherence [22]. The poor adherence to ART is a worldwide problem of striking magnitude (WHO, 2011) The WHO recommends at least 95\% of adherence to ART among PLWHA to avoid the emergence of the resistant strains of the virus [23]. Based on these facts, the importance of adhering to ART has been widely publicized and accepted as a critical element in the success of ART.

Without adequate adherence, ARV drugs are not maintained at sufficient concentrations to suppress HIV replication in infected individuals to lower the plasma viral load of HIV [24]. Additionally, poor adherence to ARV drugs accelerates development of drug-resistant HIV. The resulting virologic failure diminishes the potential risk for long-term clinical success. Drug-resistant strains of HIV can be transmitted to uninfected patients, resulting in fewer treatment options [25]. Non-adherence may eventually undermine the improvements in HIV-related health success already seen over the last years.

The current study revealed that $83.5 \%$ of the participants had high adherence to ART according to the WHO [10] cut-off point of $>95 \%$ adherence to ART. Similarly, the high adherence was reported in other studies $[12,13,15,26,17,18,27]$. Having emotional or practical support positively encouraged ART adherence, included factors related to patients and their families, socioeconomic factors, medication, and healthcare systems, stigma, information, education and communication, change in daily routine, social support and marital status among other things.

Also, high adherence and viral suppression were achieved for a significant proportion of HIVinfected patients taking ART in a resource-limited area [28]. The strategies to maximize adherence in this setting should emphasize ready access to affordable and simple ART regimens, as well as HIV education programs to help increase awareness and decrease disease stigmatization [29]. Against the expectations, sub-Saharan Africa patients have similar or higher adherence levels compared to those of developed countries [16,30]. Despite good adherence and program-related findings, ART is challenged by a range of hierarchical and interrelated factors. The challenges to ART adherence include factors related to patients and their families, socioeconomic factors, medication, and 
healthcare systems [31]. However, there is substantial room for improvement of ART programs in sub-Sahara African countries.

Conversely, low adherence to ART among PLWHA was reported by Kim et al. [13] and Achapa et al. [14]. The challenges to ART adherence were financial constraints, forgetting to take medication, lack of family care, depression, alcohol use, social stigma and side effects to ART [13,28,32], substance and alcohol abuse [26]. Thus, health care providers must identify possible barriers to adherence at the earliest possible time and provide appropriate solutions. Medication adherence leads to improved quality of life which is the key determinant of the patient's response to ART and measuring it helps to guide strategies [33].

In this study, the two variables that predicted adherence to ART were knowledge and ART clinic site. This study showed that the participants who had adequate knowledge on HIV and AIDS and ART were $73 \%$ less likely to achieve high adherence to ART compared to those who had inadequate knowledge.

Although the findings of this study have shown that the participants who had adequate knowledge on HIV and AIDS and ART were 73\% less likely to achieve high adherence to ART compared to those who had inadequate knowledge, other studies $[14,16]$ have reported contrary to our findings. Katongo [17] and Potchoo et al. [34] reported that, adherence to ART was related to stigma, information, education and communication, missing at least once a dose intake, travel, and side effects of the ARV drugs which are all influenced by knowledge.

In addition, Demessie et al. [35] reported low adherence to ART among PLWHA who had inadequate knowledge on the ART plan and regimen. Consequently, adequate knowledge on HIV and AIDS and ART regimen influences adherence to ART among PLWHA. Also, older age, use of memory aids, treatment satisfaction, and not having co-medications and regimen switch improves adherence to ART [35]. This calls for enhanced IEC both in terms of adequate dosing and improved adherence, to achieve better outcomes of the PLWHA.

Knowledge enables clients to have a better understanding of HIV and AIDS and the adherence to ARV drugs regimen [36]. Also, Vervoort et al. [37] suggested that knowledge has a positive influence on adherence to ART. Lack of knowledge on the mode of action of ARV drugs would lead to poor adherence especially if the client is not experiencing any symptom and leading a normal and healthy life [34]. The patient could therefore develop a notion that they are healed hence they can even start missing doses or even discontinue ART. Therefore clients should be able to understand that the absence of symptoms is simply because the virus has been suppressed by ART and rendered inactive.

As recently as 2010, it was estimated that $64 \%$ of $15-24$ year olds in Zambia had no knowledge about the truth of HIV and AIDS [10], showing that our peer education initiatives to combat myths and misconceptions surrounding the HIV infection and its management are still vitally needed.

This study also revealed that, the participants who attend ART clinic at Nyimba ART clinic site were 2.34 times more likely to adhere to ART compared to those at Monze ART clinic site. However, among the studies reviewed, the researchers did not come across studies that ascertained the association between ART clinic site and adherence to ART. Although, ART clinic site is one of the variables, this study was not designed to compare adherence to ART and associated factors between the two ART sites.

Thus, we may only speculate that the differences in adherence could be because the population of the PLWHA on ART visiting Nyimba ART site is larger (4005) than that of Monze ART site (3251) [38,39]. This suggests that more (200, 55\%) participants from Nyimba ART clinic site were included into the study compared to (164, 45\%) from Monze ART clinic site. However, considering the fact that both ART clinic site are rural [2] we did not expect to see much difference in terms of adherence to ART among the PLWHA from both ART clinic sites. This will be our focus in future studies.

Several studies have shown that adherence to ART is associated with various factors. The factors which were statistically significant in other studies were not significant in this study. Thus identifying 
Texila International Journal of Clinical Research

Volume 3, Issue 2, Dec 2016

the other factors influencing adherence to ART among PLWHA can improve the management of their condition.

\section{Limitations of the study}

It is possible that our findings could have been affected by many confounding factors. The possible confounding factors, such as those related to patients and their families, socioeconomic factors, medication, and healthcare systems were not adequately considered but will be the next focus in our future studies.

In addition, since this was a cross-sectional study, it is difficult to establish a "causal" relation between adherence to ART and associated factors. The other limitation was the inadequate time and cost to meet the study logistics. Also, the study was carried out on a limited study population and only the participants who visited the ART clinics during the period of data collection were considered in this study.

\section{Conclusion}

The majority of the participants in this study had high adherence to ART that was associated by knowledge and ART clinic site. This raises the concerns on the adequacy of the IEC on HIV and AIDS and ART adherence that is provided to PLWHA at Nyimba District and Monze Mission Hospitals. Considering that HIV and AIDS like most other chronic diseases is progressive, the findings suggest that if the factors influencing adherence to ART are not adequately addressed, opportunistic infections will emerge and ART regimen of the PLWHA becomes much more complex with time.

The study will enable health care providers to review their IEC and care given to PLWHA to ensure high adherence to ART. In order to achieve this, it is suggested that further research should be conducted to evaluate the type of IEC that is given to the PLWHA and to compare adherence to ART among different ART sites.

\section{Acknowledgements}

We express our sincere gratitude to the $\mathrm{MoH}$ and Ministry of Community Development Mother and Child health for sponsoring our studies, the Department of Nursing Sciences, School of Medicine, University of Zambia for the technical guidance and academic support throughout the research study. We also, thank the management of Nyimba District and Monze Mission Hospitals especially the staff in the ART clinics for the facilities and support. Lastly, our special gratitude goes to the participants for their cooperation during the study.

\section{Competing interests}

The authors declare that they have no competing interests in the study.

\section{Author's contributions}

RK, JZ and DPS conceived the study. RK, JZ, DPS and EMM designed and conducted the study. RK and EMM analysed the data and wrote the manuscript. EMM, supervised the whole study process. All the authors read and approved the final manuscript.

\section{References}

[1.] Achappa B, Madi D, Bhaskaran U, Ramapuram JT, Rao S, and Mahalingam S. Adherence to Antiretroviral Therapy Among People Living with HIV. N Am J Med Sci 2013; 5(3):220-223. doi: 10.4103/1947-2714.109196, PMCID: PMC3632027.

[2.] Birbeck GL, Kvalsund MP, Bradbury R, Mang'ombe C, Organek N. Neuropsychiatric and socioeconomic status impact antiretroviral adherence and mortality in rural Zambia, American Journal of Tropical Medicine and Hygiene 2011; 85:782-789. 
[3.] Bangsberg DR, Ware N, and Simoni JM. (2006). Adherence without access to antiretroviral therapy in sub-Saharan Africa. AIDS 2006; 20:140-1.

[4.] Carlucci JG, Kamanga A, Sheneberger R, Shepherd BE, Jenkins CA, and Spurrier J. Predictors of Adherence to Antiretroviral Therapy in Rural Zambia. JAIDS 2008; 47(5):615-22. DOI: 10.1097/QAI.0b013e318165dc25, PubMed

[5.] Central Statistical Office (CSO) [Zambia], Ministry of Health (MOH) [Zambia], and ICF International. Zambia Demographic and Health Survey 2013-14 2014; Rockville, Maryland, USA: Central Statistical Office, Ministry of Health, and ICF International.

[6.] Chesney MA. Factors Affecting Adherence to Antiretroviral Therapy. Clin Infect Dis 2000; 30 (Supplement 2): S171-S176. doi: 10.1086/313849.

[7.] DiMatteo MR. Improving adherence and quality of life through effective communication with children and their families. Patient Education and Counseling 2004; 55(3):339-344.

[8.] DiMatteo MR. Enhancing Patient Adherence to Treatment, $3^{\text {rd }}$ edition, NY: Oxford University Press 2013; 182-185.

[9.] Demessie R, Mekonnen A, Amogne W, and Shibeshi W. Knowledge and adherence to antiretroviral therapy among adult people living with HIV/AIDS at Tikur Anbessa Specialized Hospital, Ethiopia. Int J Basic Clin Pharmacol 2014; 3(2):320-330. doi: 10.5455/2319-2003.ijbcp20140413., http://www.scopemed.org/?mno=151851.

[10.] Globefeed, Zambian Distance Calculator 2014; http://www.distancecalculator,globefeed.com/Zambia.distancecalculator.asp.

[11.] Gill CJ, Hamer DH, Simon JL, Thea DM, Sabin LL. No room for complacency about adherence to antiretroviral therapy in sub-Saharan Africa. AIDS 2005; 19(12):1243-9. PubMed

[12.] Haskard YKB, and DiMatteo MR. Physician Communication and Patient Adherence to Treatment: A Meta-analysis. Med Care 2009; 47(8): 826-834. doi: 10.1097/MLR.0b013e31819a5acc, PMCID: PMC2728700, NIHMSID: NIHMS109392.

[13.] Hudelson C, and Cluver L. Factors associated with adherence to antiretroviral therapy among adolescents living with HIV/AIDS in low- and middle-income countries: a systematic review. AIDS Care 2015; 27(7): 805816. http://dx.doi.org/10.1080/09540121.2015.1011073.

[14.] Kasumu LO, amd Balogun MR. Knowledge and attitude towards antiretroviral therapy and adherence pattern of HIV patients in southwest Nigeria. Int J Infect Control 2014; v10:i3doi:10.3396/IJIC.v10i3.024.14.

[15.] Kim SH, Gerver SM, Fidler S, and Ward H. Adherence to antiretroviral therapy in adolescents living with HIV: systematic review and meta-analysis. AIDS (London, England), Lippincott Williams \& Wilkins Open Access, 2014; 28(13):1945-1956, doi: 10.1097/QAD.0000000000000316, PMCID: PMC4162330.

[16.] Ketema AK, and Weret ZS. Assessment of adherence to highly active antiretroviral therapy and associated factors among people living with HIV at Debrebrihan Referral Hospital and Health Center, Northeast Ethiopia: a cross-sectional study. HIV/AIDS-Research and Palliative Care 2015; 7:75-81. doi: 10.2147/HIV.S79328, PMC4362904.

[17.] Katongo R. Adherence to ART Among Clients with HIV/AIDS attending ART at Chinkankata Hospital 2009; Lusaka: UNZA dbase.

[18.] Kleppinger A, Litt M, Kulldorff M, Unson C, and Oat J. Health Perceptions As Predictors of Exercise Adherence in Older Women, USA, by Human Kinetics Publishers and the European College of Sport Science. European Journal of Sport Science 2003; 3(4).

[19.] Machinga EL, and Bangsberg D. Adherence to ART 2006; hiv.insit.2006:7:16-22.doi:10.1111/s14681293.2005.00337x

[20.] Mitiku H, Abdosh T, and Teklemariam Z. Factors Affecting Adherence to Antiretroviral Treatment in Harari National Regional State, Eastern Ethiopia. ISRN AIDS 2013; 7. http://dx.doi.org/10.1155/2013/960954, http://www.hindawi.com/journals/isrn/2013/960954/.

[21.] Ministry of Health. Adult and Adolescent and Antiretroviral protocols, $2^{\text {nd }}$ Edition 2010; Lusaka: Ministry of Health. 
Texila International Journal of Clinical Research

Volume 3, Issue 2, Dec 2016

[22.] Mills EJ, Bakanda C, Birungi J, et al. Life expectancy of persons receiving combination antiretroviral therapy in low-income countries: a cohort analysis from Uganda. Annals of Internal Medicine 2011; 155(4):209-217. PubMed

[23.] Menon JA, Ngoma MPS, Nkumbula T, Paul R, and Sichimba S. Gaps in the Implementation of AntiRetroviral Treatment: A Case for Addressing Gender and Mental Health Consequences of HIV Positive Individuals. Medical Journal of Zambia 2014; 41(3).

[24.] Mitty JA, Stone VE, Sands M. et al. Directly observed therapy for the treatment of people with human immunodeficiency virus infection: a work in progress. Clin Infect Dis 2002; 34984-990.990. PubMed

[25.] Mweemba P, Makukula MK, Mukwato PK, and Makoleka MM. Quality of Life and Adherence to Antiretroviral Drugs. Medical Journal of Zambia 2010; 37(1).

[26.] Monze Mission Hospital. Monze Mission Hospital Action Plan 2014-2015. 2013; Monze: Monze Mission Hospital.

[27.] Nsakanya R. Factors affecting ART adherence in Choma 2010; Lusaka. UNZA dbase.

[28.] Nachega JB, Stein DM, Lehman DA, Hlatshwayo D, Mothopeng R, Chaisson RE, et al. Adherence to Antiretroviral Therapy in HIV-Infected Adults in Soweto, South Africa. AIDS Research and Human Retroviruses 2009; 20(10):1053-1056. doi:10.1089/aid.2004.20.1053. http://online.liebertpub.com/doi/abs/10.1089/aid.2004.20.1053.

[29.] Nyimba District Hospital. Nyimba District Hospital Action Plan 2014-2015. 2013; Monze: Nyimba District Hospital.

[30.] Oladipo DO, and Ogundhunsi O. Default rate from ART program in Nigeria. JAHR 2013; www.Bioline.org.br.pdf?md080.Org/jahr.

[31.] Potchoo Y, Tchamdja K, Balogou A, Pitche VP, Guissou IP and Kassang EK. Knowledge and adherence to antiretroviral therapy among adult people living with HIV/AIDS treated in the health care centers of the association "Espoir Vie Togo" in Togo, West Africa. BMC Clin Pharmacol 2010; 10(11). doi: 10.1186/14726904-10-11, PMCID: PMC2949664.

[32.] Reda AA, and Biadgilign S. Determinants of Adherence to Antiretroviral Therapy among HIV-Infected Patients in Africa. AIDS Res Treat, 2012; 574656. doi: 10.1155/2012/574656, PMCID: PMC3296173.

[33.] Stone VE, Hogan JW, Schuman P, et al. Antiretroviral regimen complexity, self-reported adherence, and HIV patients' understanding of their regimens: Survey of women in the HER study,” Journal of Acquired Immune Deficiency Syndromes 2001; 28(2), 124-131. Scopus | Google Scholar

[34.] Vervoort SCJM, Borleffs JCC, Hoepelman AIM, and Grypdonck MHF. Adherence in antiretroviral therapy: a review of qualitative studies. AIDS 2007; 21(3):271-281, doi: 10.1097/QAD.0b013e328011cb20.

[35.] World Health Organization. HIV/AIDS Fact sheet No 360, 2015; Geneva: World Health Organization http://www.who.int/mediacentre/factsheets/fs360/en/.

[36.] World Health Organization. Improving medication adherence, 2010; Geneva: World Health Organization. [37.] Wasti SP, van Teijlingen E, Simkhada P, Randall J, Baxter S, Kirkpatrick P, et al. Factors influencing adherence to antiretroviral treatment in Asian developing countries: a systematic review. Tropical Medicine \& International Health 2012; 17(1), 17-81. Google Scholar

[38.] World Health Organization. Medication Adherence-WHO Cares 2011; Geneva: World Health Organization.

[39.] Wang H, Zhou J, He G, Luo Y, Li X, Yang A. et al. Consistent ART Adherence Is Associated with Improved Quality of Life, CD4 Counts, and Reduced Hospital Costs in Central China. AIDS Res Hum Retroviruses 2009; 25(8):757-763. doi:10.1089/aid.2008.0173, PMCID: PMC2858929.

Table 1: Adherence to ART by socio-demographic factors of participants

\begin{tabular}{|c|c|c|c|}
\hline & Adheren & to ART & \\
\hline & $\begin{array}{c}\text { High }(n=304,>95 \% \\
\text { adherence) }\end{array}$ & $\begin{array}{c}\text { Low }(\mathrm{n}=60,<95 \% \\
\text { adherence) }\end{array}$ & \\
\hline Characteristic & No (\%) & No (\%) & $P$-value* \\
\hline
\end{tabular}




\begin{tabular}{lccc} 
18-25 years & $73(88.0)$ & $10(12.0)$ & \\
$26-35$ years & $147(85.5)$ & $25(14.5)$ & $0.173^{\mathrm{a}}$ \\
$36-45$ years & $55(77.5)$ & $16(22.5)$ & \\
$<46$ years & $29(76.3)$ & $9(23.7)$ & \\
Sex & & & \\
Male & $125(84.5)$ & $23(15.5)$ & \\
Female & $179(82.9)$ & $37(17.1)$ & $0.688^{\mathrm{a}}$ \\
Marital status & & & \\
Single & $197(86.0)$ & $32(13.9)$ & $0.209^{\mathrm{b}}$ \\
Married & $107(79.3)$ & $28(20.7)$ & \\
Religion & & & \\
Christian & $184(82.9)$ & $38(17.1)$ & \\
Non-Christian & $120(84.5)$ & $22(15.5)$ & \\
Education & & & \\
Never/Primary & $223(83.2)$ & $45(16.8)$ & \\
Secondary & $51(86.4)$ & $8(13.6)$ & \\
College/University & $30(81.1)$ & $7(18.9)$ & \\
Employment & & & \\
Formal & $148(84.1)$ & $28(15.9)$ & \\
Self employed & $99(81.1)$ & $10(15.2)$ & \\
Unemployed & $56(84.8)$ & $42(21.0)$ & \\
ART site & & $18(11.0)$ & \\
Nyimba & $158(79.0)$ & & \\
Monze & $146(89.0)$ & & \\
\hline$* P<0.05 .{ }^{a}$ Pearson's Chi-squared test, ${ }^{\mathrm{b}}$ Fisher’s exact test & \\
\hline & & & \\
\hline
\end{tabular}

Table 2: Adherence to ART by factors of the participants

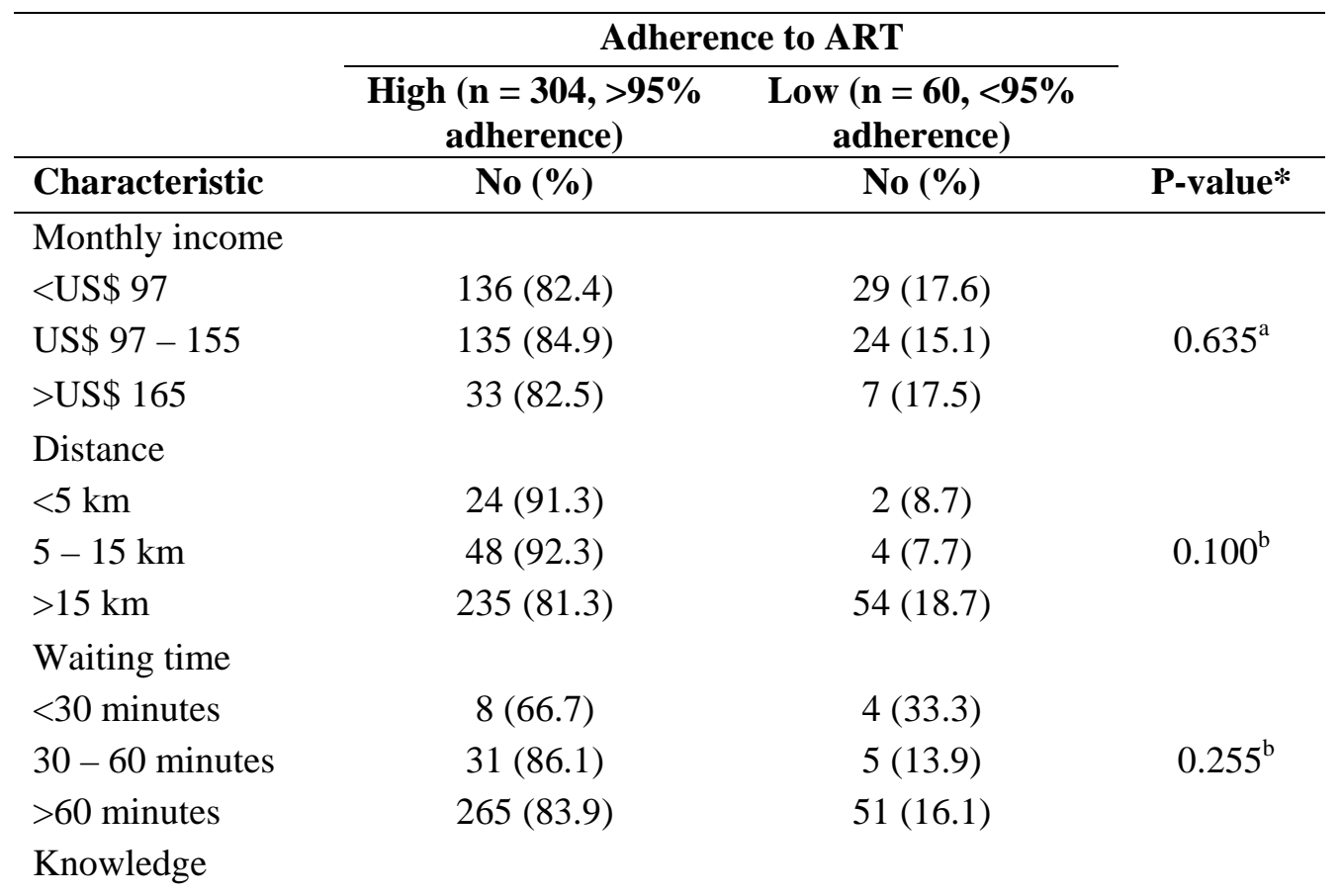


Texila International Journal of Clinical Research

Volume 3, Issue 2, Dec 2016

\begin{tabular}{lccc} 
Adequate & $269(86.2)$ & $43(13.8)$ & \\
Inadequate & $35(67.3)$ & $17(32.7)$ & $0.001^{\mathrm{a}}$ \\
$\begin{array}{l}\text { Health status } \\
\text { Good }\end{array}$ & $264(83.0)$ & $54(17.0)$ & \\
Poor & $40(87.0)$ & $6(13.0)$ & $0.501^{\mathrm{a}}$ \\
Staff attitude & & & \\
Good & $167(79.7)$ & $42(20.1)$ & \\
Poor & $137(88.4)$ & $18(11.6)$ & $0.033^{\mathrm{a}}$ \\
Client attitude & & \\
Good & $276(83.1)$ & $56(16.9)$ & \\
Poor & $28(87.5)$ & $4(12.5)$ & $0.626^{\mathrm{a}}$ \\
\hline${ }^{*} P<0.05 .{ }^{\mathrm{a}}$ Pearson's Chi-Squared Test, ${ }^{\mathrm{b}}$ Fisher's Exact Test & \\
\hline
\end{tabular}

Table 3: Multivariate binary logistic regression model-determining factors associated with adherence to ART

\begin{tabular}{|c|c|c|c|c|}
\hline & \multicolumn{2}{|c|}{ Adherence to ART } & & \\
\hline & $\begin{array}{c}\text { High }(\mathbf{n}=304 \\
>95 \% \\
\text { adherence })\end{array}$ & $\begin{array}{c}\text { Low }(\mathbf{n}=60, \\
>95 \% \\
\text { adherence) }\end{array}$ & & \\
\hline Predictor variable & No (\%) & No (\%) & $\operatorname{AOR}(95 \% \text { CI })^{\mathrm{a}}$ & P-value* \\
\hline \multicolumn{5}{|l|}{ Staff attitude } \\
\hline Good & 167 (79.7) & $42(20.1)$ & 1.78 (0.96-3.30) & 0.069 \\
\hline Poor & 137 (88.4) & $18(11.6)$ & Ref (1.00) & \\
\hline \multicolumn{5}{|l|}{ Knowledge } \\
\hline Adequate & 269 (86.2) & 43 (13.8) & $0.27(0.14-0.54)$ & 0.000 \\
\hline Inadequate & 35 (67.3) & $17(32.7)$ & Ref (1.00) & \\
\hline \multicolumn{5}{|l|}{ ART site } \\
\hline Nyimba & $158(79.0)$ & $42(21.0)$ & $2.34(1.25-4.40)$ & 0.033 \\
\hline Monze & $146(89.0)$ & $18(11.0)$ & Ref (1.00) & \\
\hline
\end{tabular}




\title{
Acute kidney Injury: Prevalence, Diagnosis, Causes and treatment
}

\author{
Article by Alermayehu Lelisa Duga \\ M.Sc clinical Research, Texila American University, Swaziland \\ Email:aduga@texilaconnect.com
}

\begin{abstract}
Introduction: Acute Kidney injury (AKI), is defined as an abrupt reduction in kidney function measured by a rapid decline in glomerular filtration rate resulting in the retention of metabolic waste products and dysregulation of fluid, electrolyte, and acid-base homeostasis. AKI is also defined in terms of a rise in serum creatinine concentration or by azotemia (a rise in blood urea nitrogen [BUN] concentration). The causes of acute renal disease can be related to the factors that interfere with structure and function of renal arteries, glomerular, renal tubules and urinary tracts. The current treatment for AKI is mainly supportive in nature; no therapeutic modalities to date have shown efficacy in treating the condition. Maintenance of volume homeostasis and correction of biochemical abnormalities remain the primary goals of treatment.

Methods: I undertook a systemic review different books and journals and came up with summary statement. Accordingly supporting evidence and general recommendation was generated for the future research.

Result: using the literature review the definition, causes and treatment algorithm of acute kidney injury was jotted down.

Conclusion: Acute kidney injury (formerly known as acute renal failure) is a syndrome characterised by the rapid loss of the kidney's excretory function and is typically diagnosed by the accumulation of end products of nitrogen metabolism (urea and creatinine) or decreased urine output, or both.
\end{abstract}

Keywords: Acute renal failure, creatinine, kidney, acute kidney injury, urea, blood urea nitrogen, dialysis.

\section{Introduction}

The kidneys are two organs located in the midsection on either side of spine in the middle of human back, just above the waist. They clean blood, keep the balance of salt and minerals in the blood, and help control blood pressure. When kidneys are damaged, waste products and fluid can build up in the body, causing swelling in ankles, vomiting, weakness, poor sleep, and shortness of breath(1). Acute Kidney injury (AKI), sometimes called acute renal failure (ARF), is defined as an abrupt reduction in kidney function measured by a rapid decline in glomerular filtration rate resulting in the retention of metabolic waste products and dysregulation of fluid, electrolyte, and acid-base homeostasis $(2,3)$.

AKI is usually defined in terms of a rise in serum creatinine concentration or by azotemia (a rise in blood urea nitrogen [BUN] concentration) (4). However, immediately after a kidney injury, BUN or creatinine levels may be normal, and the only sign of a kidney injury may be decreased urine production. A rise in the creatinine level can result from medications (eg, cimetidine, trimethoprim) that inhibit the kidney's tubular secretion, while a rise in the BUN level can also occur without renal injury, resulting instead from such sources as gastrointestinal (GI) or mucosal bleeding, steroid use, or protein loading. Therefore, a careful inventory must be taken before concluding that a kidney injury is present. Therefore, the definition of Acute Kidney injury remain subject to controversy, confusion and lack of consensus. (5). 
To address the lack of a universal definition for AKI a collaborative network of international experts representing nephrology and intensive care societies established the Acute Dialysis Quality Initiative (ADQI) and devised the RIFLE definition and staging system for AKI. Shortly after this many of the original members of the ADQI group collaborated to form the Acute Kidney Injury Network (AKIN) $(6,7)$. The AKIN group modified the RIFLE staging system to reflect the clinical significance of relatively small rises in serum creatinine. Most recently the international guideline group, Kidney Disease: Improving Global Outcomes (KDIGO) has brought together international experts from many different specialties to produce a definition and staging system that harmonises the previous definitions and staging systems proposed by both ADQI and AKIN. It is anticipated that this definition and staging system will be adopted globally. This will enable future comparisons of the incidence, outcomes and efficacy of therapeutic interventions for AKI (8).

\section{Pathophysiology of AKI}

Acute kidney injury (AKI) is associated with prolonged hospitalization, substantial health care resource consumption, high mortality, and can lead to progressive chronic kidney disease (CKD), including chronic kidney failure, in survivors(9). The causes of acute renal disease can be related to the renal anatomy most affected by the disorder as follows:

- Vascular - Blood from the renal arteries is delivered to the glomeruli.

- Glomeruli - Ultrafiltration occurs at the glomeruli forming an ultrafiltrate, which subsequently flows into the renal tubules.

- Renal tubule - Reabsorption and secretion of solute and/or water from the ultrafiltrate occurs within the tubules.

- Urinary tract - The final tubular fluid, the urine, leaves the kidney, draining sequentially into the renal pelvis, ureter, and bladder, from which it is excreted through the urethra.

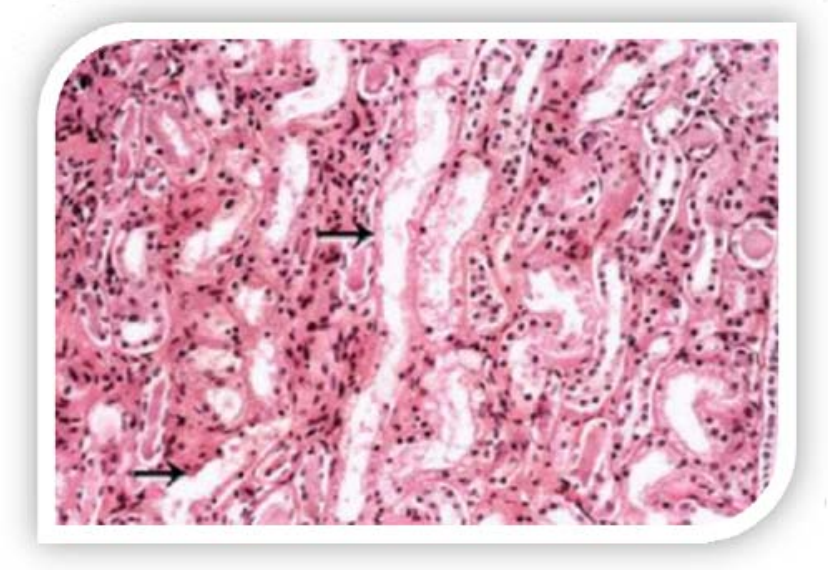

Fig. 1. Microscopic picture of a renal biopsy specimen shows renal medulla, which is composed mainly of renal tubules. Patchy or diffuse denudation of the renal tubular cells with loss of brush border is observed, suggesting acute tubular necrosis as the cause of acute renal failure(10).

\section{Classifications of AKI}

Any process that interferes with any of the structures and/or functions can cause renal disease. The causes of AKI can therefore be categorized as prerenal, renal, or postrenal(11) 


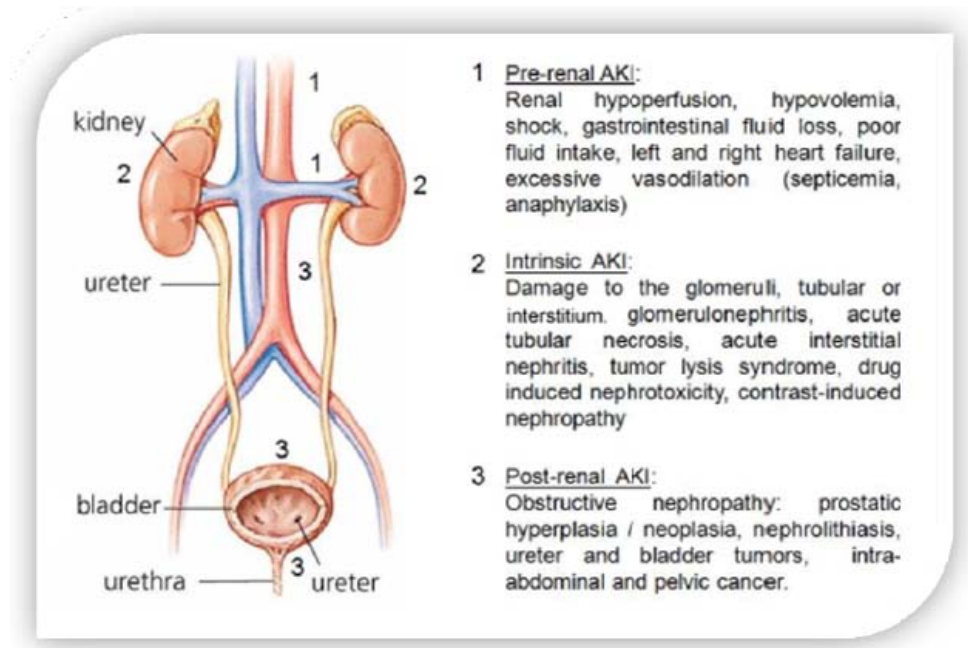

Fig.2 The brief Classifications of RKI

Table 2 classification and major causes of AKI

\section{A. Prerenal ARF}

I. Hypovolemia

A. Increased extracellular fluid losses: hemorrhage

B. Gastrointestinal fluid loss: vomiting, diarrhea, enterocutaneous fistula

C. Renal fluid loss: diuretics, osmotic diuresis, hypoadrenalism, nephrogenic diabetes insipidus

D. Extravascular sequestration: burns, pancreatitis, severe hypoalbuminemia (hypoproteinemia)

E. Decreased intake: dehydration, altered mental status

II. Altered renal hemodynamics resulting in hypoperfusion

A. Low cardiac output state: diseases of the myocardium, valves, and pericardium (including tamponade); pulmonary hypertension or massive pulmonary embolism leading to right and left heart failure; impaired venous return (e.g., abdominal compartment syndrome or positive pressure ventilation)

B. Systemic vasodilation: sepsis, antihypertensives, afterload reducers, anaphylaxis

C. Renal vasoconstriction: hypercalcemia, catecholamines, calcineurin inhibitors, amphotericin $\mathrm{B}$

D. Impairment of renal autoregulatory responses: cyclooxygenase inhibitors (e.g., nonsteroidal anti-inflammatory drugs), angiotensin-converting enzyme inhibitors, or angiotensin II receptor blockers

E. Hepatorenal syndrome

B. Intrinsic ARF

I. Renovascular obstruction (bilateral, or unilateral in the setting of one kidney)

A. Renal artery obstruction: atherosclerotic plaque, thrombosis, embolism, dissection aneurysm, large vessel vasculitis

B. Renal vein obstruction: thrombosis or compression

II. Diseases of the glomeruli or vasculature

A. Glomerulonephritis or vasculitis

B. Other: thrombotic microangiopathy, malignant hypertension, collagen vascular diseases (systemic lupus erythematosus, scleroderma), disseminated intravascular coagulation, preeclampsia

III. Acute tubular necrosis

A. Ischemia: causes are the same as for prerenal ARF, but generally the insult is more severe and/or more prolonged

B. Infection, with or without sepsis syndrome 
C. Toxins:

1. Exogenous: radiocontrast, calcineurin inhibitors, antibiotics (e.g., aminoglycosides), chemotherapy (e.g., cisplatin), antifungals (e.g., amphotericin B), ethylene glycol

2. Endogenous: rhabdomyolysis, hemolysis

IV. Interstitial nephritis

A. Allergic: antibiotics ( $\quad$-lactams, sulfonamides, quinolones, rifampin), nonsteroidal antiinflammatory drugs, diuretics, other drugs

B. Infection: pyelonephritis (if bilateral)

C. Infiltration: lymphoma, leukemia, sarcoidosis

D. Inflammatory, nonvascular: Sjögren's syndrome, tubulointerstitial nephritis with uveitis

V. Intratubular obstruction

A. Endogenous: myeloma proteins, uric acid (tumor lysis syndrome), systemic oxalalosis

B. Exogenous: acyclovir, gancyclovir, methotrexate, indinavir

C. Postrenal ARF (Obstruction)

I. Ureteric (bilateral, or unilateral in the case of one kidney): calculi, blood clots, sloughed papillae, cancer, external compression (e.g., retroperitoneal fibrosis)

II. Bladder neck: neurogenic bladder, prostatic hypertrophy, calculi, blood clots, cancer

III. Urethra: stricture or congenital valves

Intrinsic renal disorders - Intrinsic renal disease includes disorders that involve the renal vascular, glomerular, and/or tubular/interstitial pathology.

Vascular - Vascular causes of AKI include thrombosis (arterial and venous), hemolytic-uremic syndrome, malignant hypertension, and vasculitis.

Glomerular - The principal glomerular cause of AKI is acute glomerulonephritis, which is commonly postinfectious. AKI can be observed with most of the glomerulonephritides that can occur in childhood.

Tubular and interstitial disease - Acute tubular necrosis (ATN) results from ischemia due to decreased renal perfusion or injury from tubular nephrotoxins. All causes of prerenal azotemia can progress to ATN if renal perfusion is not restored and/or nephrotoxic insults are not withdrawn.

The administration of nephrotoxic agents, including aminoglycosides, amphotericin $\mathrm{B}$, and contrast agents, is a common cause of tubular disease. AKI can also be induced by the release of heme pigments, as with myoglobinuria due to rhabdomyolysis and hemoglobinuria due to intravascular hemolysis.

In children, acute interstitial nephritis most commonly results from a reaction to a drug that is thought to be hypersensitive in nature.

Postrenal - Postrenal AKI is due to bilateral urinary tract obstruction unless there is a solitary kidney. In neonates, urinary tract obstruction, due to posterior urethral valves is the most common cause of postrenal failure. Children with chronic obstructive uropathies are also at significant increased risk of AKI from ischemic and toxic insults(12).

\section{RIFLE classification system}

The Acute Dialysis Quality Initiative work group RIFLE (Risk of renal dysfunction, Injury to the kidney, Failure or Loss of kidney function) coined a definition and classification system for acute renal failure. 
Table 2 RIFLE classification system

\begin{tabular}{|c|c|c|c|}
\hline Stage & GFR Criteria & Urine Output Criteria & Probability \\
\hline Risk & $\begin{array}{l}\text { SCr increased } \times 1.5 \\
\text { Or } \\
\text { GFR decreased }>25 \%\end{array}$ & $\mathrm{UO}<0.5 \mathrm{~mL} / \mathrm{kg} / \mathrm{h} \times 6 \mathrm{~h}$ & \multirow[t]{3}{*}{$\begin{array}{l}\text { High sensitivity (Risk } \\
>\text { Injury >Failure) }\end{array}$} \\
\hline Injury & $\begin{array}{l}\text { SCreat increased } \times 2 \\
\text { or } \\
\text { GFR decreased }>50 \%\end{array}$ & $\mathrm{UO}<0.5 \mathrm{~mL} / \mathrm{kg} / \mathrm{h} \times 12 \mathrm{~h}$ & \\
\hline Failure & $\begin{array}{l}\text { SCreat increased } \times 3 \\
\text { or } \\
\text { GFR decreased } 75 \% \\
\text { or } \\
\text { SCreat } \geq 4 \mathrm{mg} / \mathrm{dL} \text {; acute } \\
\text { rise } \geq 0.5 \mathrm{mg} / \mathrm{dL}\end{array}$ & $\begin{array}{l}\mathrm{UO}<0.3 \mathrm{~mL} / \mathrm{kg} / \mathrm{h} \times 24 \mathrm{~h} \\
\text { (oliguria) } \\
\text { Or } \\
\text { anuria } \times 12 \mathrm{~h}\end{array}$ & \\
\hline Loss & \multicolumn{2}{|c|}{$\begin{array}{l}\text { Persistent acute renal failure: complete loss of kidney } \\
\text { function }>4 \mathrm{wk}\end{array}$} & \multirow[t]{2}{*}{ High specificity } \\
\hline ESKD* & \multicolumn{2}{|c|}{ Complete loss of kidney function $>3 \mathrm{mo}$} & \\
\hline
\end{tabular}

\section{Acute kidney injury network classification system}

The Acute Kidney Injury Network (AKIN) has developed specific criteria for the diagnosis of AKI. The AKIN defines AKI as abrupt (within 48 hours) reduction of kidney function, manifested by any 1 of the following (13). An absolute increase in serum creatinine of $0.3 \mathrm{mg} / \mathrm{dL}$ or greater $(\geq 26.4$ $\mu \mathrm{mol} / \mathrm{L})$

- A percentage increase in serum creatinine of $50 \%$ or greater (1.5-fold from baseline)

- A reduction in urine output, defined as less than $0.5 \mathrm{~mL} / \mathrm{kg} / \mathrm{h}$ for more than 6 hours

AKIN has proposed a staging system for AKI that is modified from RIFLE. In this system, either serum creatinine or urine output criteria can be used to determine stage according to the following Table.

Table 3 Acute Kidney Injury Network Classification/Staging System for AKI

\begin{tabular}{|l|l|l|}
\hline Stage & Serum Creatinine Criteria & Urine Output Criteria \\
\hline $\mathbf{1}$ & $\begin{array}{l}\text { Increase of } \geq 0.3 \mathrm{mg} / \mathrm{dL}(\geq 26.4 \mu \mathrm{mol} / \mathrm{L}) \text { or } \\
\text { 1.5- to } 2 \text {-fold increase from baseline }\end{array}$ & $<0.5 \mathrm{~mL} / \mathrm{kg} / \mathrm{h}$ for $>6 \mathrm{~h}$ \\
\hline $\mathbf{2}$ & $>2$-fold to 3-fold increase from baseline & $<0.5 \mathrm{~mL} / \mathrm{kg} / \mathrm{h}$ for $>12 \mathrm{~h}$ \\
\hline $3^{*}$ & $\begin{array}{l}>3 \text {-fold increase from baseline, or increase of } \\
\geq 4.0 \mathrm{mg} / \mathrm{dL}(\geq 35.4 \mu \mathrm{mol} / \mathrm{L}) \text { with an acute } \\
\text { increase of at least } 0.5 \mathrm{mg} / \mathrm{dL}(44 \mu \mathrm{mol} / \mathrm{L})\end{array}$ & $\begin{array}{l}<0.3 \mathrm{~mL} / \mathrm{kg} / \mathrm{h} \text { for } 24 \mathrm{~h} \text { or anuria } \\
\text { for } 12 \mathrm{~h}\end{array}$ \\
\hline
\end{tabular}

\section{Epidemiology of AKI}

Although the overall precise incidence and prevalence of AKI has been difficult to ascertain, a retrospective review from England estimated a yearly incidence for AKI in children as 0.8 per 100,000 populations (14). This incidence is about one-fifth of that found in adults. There are 
indications that the overall incidence of renal failure in children is rising with increased availability of advanced pediatric medical technology including bone marrow, hepatic, and cardiac transplantation, congenital heart disease surgery, and in the care of the very low birth weight infants (15). In a retrospective review of a tertiary center in the United States, one-third of hospitalized non-critically ill children developed AKI based on the modified pediatric RIFLE criteria (16). Risk factors for AKI included increasing length of hospital stay and costs, and exposure to nephrotoxic medications.

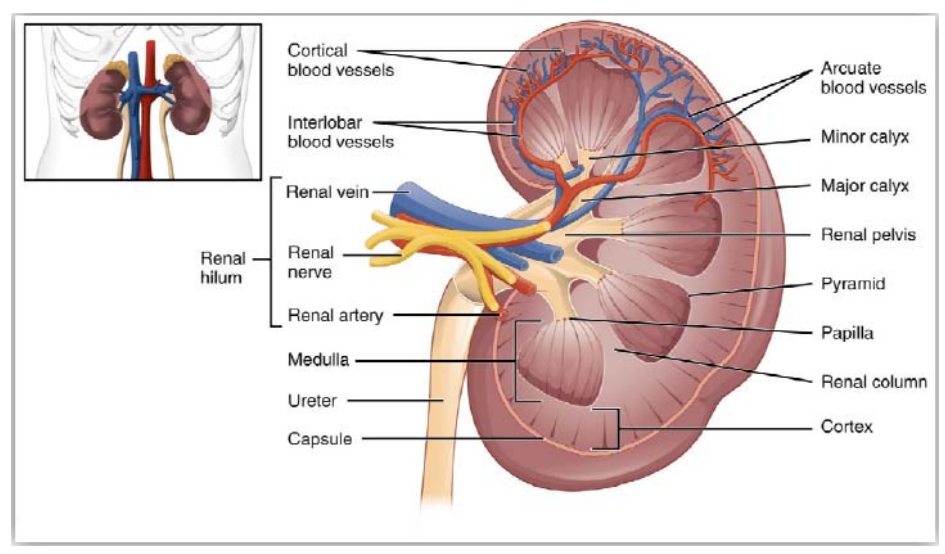

Figure 3 the image of kidney

Causes of AKI - As the prevalence of AKI has changed with the advances in medicine, so has the prevalence of different causes of AKI changed. This is especially true in tertiary care centers in developed countries where many children with pre-existing chronic diseases are at increased risk for AKI due to ischemia, drug toxicity, or infection (17).

This is illustrated in the following retrospective study of 248 patients cared for at a single tertiary referral center from 1998 to 2001 in the United States (18). Approximately two-thirds had an underlying comorbid condition.

- The most common causes of acute kidney injury (AKI) included ischemia (21 percent), nephrotoxic drugs (16 percent), sepsis (11 percent), and unknown (11 percent).

- Primary renal diseases accounted for only 7 percent of cases (17 cases) and included glomerulonephritis (9 cases), pyelonephritis (5 cases), and hemolytic uremic syndrome (3 cases).

- A quarter of the patients were neonates ( 0 to 30 days of age). In 27 percent of this group, AKI was caused by ischemia due to congenital cardiac disease.

In contrast, primary renal diseases are more likely to cause AKI in less developed countries (19).

This was best illustrated in a review of seven studies primarily from Asia (India [3 reports], New Zealand [1 trial], Singapore [1 trial]) that reported an incidence of primary renal disease (43 percent) causing ARF (ie, requiring dialysis therapy) in children. The following were the most common causes of ARF ranked in order of incidence:

- Acute tubular necrosis (ATN), (23 percent)

- Hemolytic uremic syndrome (HUS), (21 percent)

- Glomerulonephritis (13 percent)

- Intrinsic renal disease (9 percent), causes not specified

- $\quad$ Postoperative (7 percent)

- $\quad$ Sepsis (6 percent)

- Ischemia/prerenal (4.5 percent)

- Urinary tract obstruction (3 percent)

- Miscellaneous causes (13.8 percent) including metabolic disorders, renal venous thrombosis, hepatorenal syndrome, complication of organ transplantation 
Table 4 The Phases of AKI

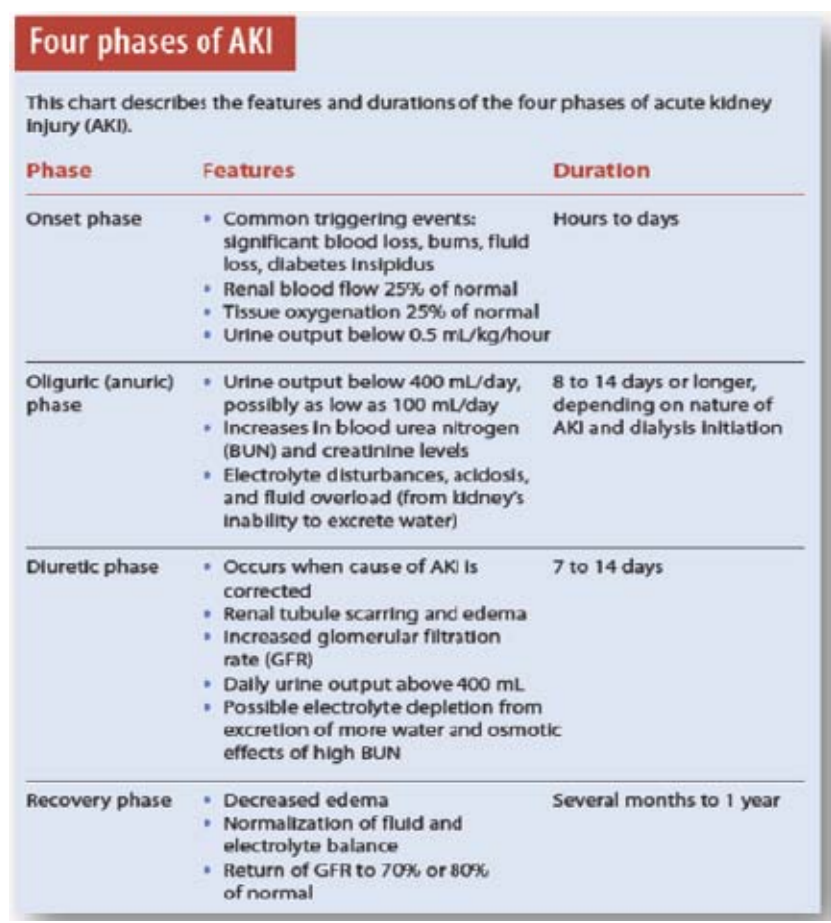

A case series of 311 children cared for at a single tertiary center in Thailand from 1982 to 2004 also demonstrated primary renal disease was more likely to cause AKI (20). In this report, the etiology of AKI included sepsis (21 percent), hypovolemia (12 percent), poststreptococcal glomerulonephritis (12 percent), systemic lupus erythematosus (10 percent), and other infectious diseases ( 9 percent).

In these studies, the most common cause of AKI in children was ATN due to either decreased renal perfusion (due to ischemia and sepsis) or nephrotoxic agents. In children, hypovolemia from excessive fluid losses from vomiting, diarrhea, burns, trauma is the usual cause of decreased renal perfusion (21).

\section{Clinical presentation}

A careful history and physical examination can frequently identify events and/or disease processes that underlie AKI and suggest an underlying diagnosis:

- A history of vomiting, diarrhea, hemorrhage, sepsis and/or decreased oral intake resulting in hypovolemia, associated with decreased urine output suggests AKI due to prerenal disease or ATN.

- Physical examination findings that include tachycardia, dry mucous membranes, sunken eyes, orthostatic blood pressure changes, and decreased skin turgor suggest hypovolemia, resulting in AKI due to prerenal disease or ATN.

- Bloody diarrhea with oliguria (defined as less than $500 \mathrm{~mL} / 1.73 \mathrm{~m} 2$ per day in children and less than $0.5 \mathrm{~mL} / \mathrm{kg}$ per hour in infants) or anuria (absent urine) is consistent with the hemolytic-uremic syndrome.

- A history of pharyngitis or impetigo, a few weeks prior to the onset of gross hematuria suggests post-infectious glomerulonephritis.

- Nephrotic syndrome, heart failure, and liver failure may result in edema and other signs of specific organ dysfunction.

- Hemoptysis in the presence of renal impairment suggests a diagnosis of pulmonary-renal syndrome, which includes Goodpasture's syndrome or granulomatosis with polyangiitis (formerly referred to as Wegener's granulomatosis) 
Texila International Journal of Clinical Research

Volume 3, Issue 2, Dec 2016

- Skin findings, such as purpura, malar rash, or petechiae, and/or joint pain favor a diagnosis of systemic vasculitis, such as systemic lupus erythematosus or Henoch Schönleinpurpura.

- Anuria or oliguria in a newborn suggests a major congenital malformation or genetic disease, such as posterior urethral valves, bilateral renal vein thrombosis, or autosomal recessive kidney disease.

- In the hospital, ATN resulting from hypotension (due to sepsis or intraoperative events) or from the administration of nephrotoxic medications (such as aminoglycosides or amphotericin-B) is the common cause of AKI (22).

Among patients who develop AKI in the hospital, the day of onset can be determined in the patient in whom the serum creatinine concentration is measured daily. Suppose, for example, that a child has had a stable serum creatinine concentration, which then begins to rise progressively on day five. In such a patient, there must have been some insult on day four or a cumulative insult that has become clinically apparent (most often aminoglycoside therapy). Careful perusal of the patient's chart may identify the precipitating event on day four (eg, hypotension, radiocontrast exposure).

\section{Evaluation and diagnosis}

In addition to a careful history and physical examination, the initial evaluation includes an estimation of the glomerular filtration rate, examination of the urine, and the use of other modalities.

Table 5 The metric Units used in evaluation and diagnosis of AKI

CONVERSION FACTORS OF METRIC UNITS TO SI UNITS

\begin{tabular}{lccc}
\hline Parameter & Metric units & Conversion factor & Sl units \\
\hline Amikacin (serum, plasma) & $\mu g / \mathrm{ml}$ & 1.708 & $\mu \mathrm{mol} /$ \\
Blood urea nitrogen & $\mathrm{mg} / \mathrm{dl}$ & 0.357 & $\mathrm{mmol} /$ \\
Calcium, ionized (serum) & $\mathrm{mg} / \mathrm{dl}$ & 0.25 & $\mathrm{mmol} / /$ \\
Creatinine (serum) & $\mathrm{mg} / \mathrm{dl}$ & 88.4 & $\mu \mathrm{mol} / /$ \\
Creatinine clearance & $\mathrm{m} / \mathrm{min}$ & 0.01667 & $\mathrm{~m} / \mathrm{s}$ \\
Gentamicin (serum) & $\mu \mathrm{g} / \mathrm{ml}$ & 2.09 & $\mu \mathrm{mol} /$ \\
Glucose & $\mathrm{mg} / \mathrm{dl}$ & 0.0555 & $\mathrm{mmol} / /$ \\
Lactate (plasma) & $\mathrm{mg} / \mathrm{dl}$ & 0.111 & $\mathrm{mmol} / 1$ \\
Tobramycin (serum, plasma) & $\mu g / \mathrm{ml}$ & 2.139 & $\mu \mathrm{mol} /$ \\
Urea (plasma) & $\mathrm{mg} / \mathrm{ml}$ & 0.167 & $\mathrm{mmol} / /$ \\
\hline
\end{tabular}

Note: Metric unit $\times$ conversion factor $=$ SI unit.

Serum creatinine concentration - Estimation of the glomerular filtration rate (GFR) gives an approximate number of functioning nephrons. Serum creatinine concentration is used clinically to estimate GFR. However, serum creatinine and estimation of GFR can only be used in patient with stable kidney function. With AKI, GFR is initially markedly reduced but there has not been time for creatinine to accumulate and for serum creatinine to accurately reflect the degree of renal function. In the setting of AKI, an elevated serum creatinine is usually observed but it has no diagnostic utility in the estimation of GFR. In addition, changes in serum creatinine can be used to clinically follow the course of disease. The normal range of serum creatinine concentrations varies by age in children as follows (23).

- Newborn - 0.3 to $1.0 \mathrm{mg} / \mathrm{dL}$ (27 to $88 \mathrm{micromol} / \mathrm{L}$ )

- Infant -0.2 to $0.5 \mathrm{mg} / \mathrm{dL}$ (18 to $35 \mathrm{micromol} / \mathrm{L})$

- Child - 0.3 to $0.7 \mathrm{mg} / \mathrm{dL}$ (27 to $62 \mathrm{micromol} / \mathrm{L}$ )

- Adolescent - 0.5 to $1.0 \mathrm{mg} / \mathrm{dL}$ (44 to $88 \mathrm{micromol} / \mathrm{L}$ )

Several formulas that utilize easily obtained values have been developed that help estimate the GFR in patients with chronic renal failure that have stable function and are discussed separately.

Urinalysis - The urinalysis is the most important noninvasive test in the diagnostic evaluation, since characteristic findings on microscopic examination of the urine sediment strongly suggest certain diagnoses (table $\underline{6}$ ). 
Table 6. Showing Correlation between urinary patterns and renal disease

\begin{tabular}{|l|l|}
\hline Urinary pattern & Renal disease \\
\hline $\begin{array}{l}\text { Hematuria with red cell } \\
\text { casts, dysmorphic red cells, } \\
\text { heavy proteinuria, or } \\
\text { lipiduria }\end{array}$ & Virtually diagnostic of glomerular disease or vasculitis \\
\hline $\begin{array}{l}\text { Multiple granular and } \\
\text { epithelial cell casts with } \\
\text { free epithelial cells }\end{array}$ & $\begin{array}{l}\text { Strongly suggestive of acute tubular necrosis in a patient with } \\
\text { acute renal failure }\end{array}$ \\
\hline $\begin{array}{l}\text { Pyuria with white cell and } \\
\text { granular or waxy casts and } \\
\text { no or mild proteinuria }\end{array}$ & $\begin{array}{l}\text { Suggestive of tubular or interstitial disease or urinary tract } \\
\text { Obstruction }\end{array}$ \\
\hline $\begin{array}{l}\text { Hematuria and pyuria with } \\
\text { no or variable casts } \\
\text { (excluding red cell casts) }\end{array}$ & $\begin{array}{l}\text { May be observed in acute interstitial nephritis, glomerular } \\
\text { disease, vasculitis, obstruction, and renal infarction }\end{array}$ \\
\hline Hematuria alone & Varies with the clinical setting \\
\hline Pyuria alone & $\begin{array}{l}\text { Usually infection; sterile pyuria suggests urinary tract } \\
\text { tuberculosis or tubulointerstitial disease }\end{array}$ \\
\hline $\begin{array}{l}\text { Few cells with little or no } \\
\text { casts or proteinuria } \\
\text { (normal or near-normal) }\end{array}$ & $\begin{array}{l}\text { In acute renal failure, prerenal disease, urinary tract } \\
\text { obstruction, hypercalcemia, myeloma kidney, some cases of } \\
\text { acute tubular necrosis, or a vascular disease with glomerular } \\
\text { ischemia but not infarction (scleroderma, atheroemboli); in } \\
\text { chronic renal failure, nephrosclerosis, urinary tract } \\
\text { obstruction, and tubulointerstitial disease }\end{array}$ \\
\hline
\end{tabular}

As examples:

- A normal or near-normal urinalysis, characterized by few cells with little or no casts or proteinuria, suggests prerenal disease, urinary tract obstruction, and some cases of acute tubular necrosis (ATN).

- Muddy brown granular casts and epithelial cell casts are highly suggestive of ATN. However, the absence of these urinary findings does not exclude the diagnosis.

- The finding of a red cell cast is diagnostic of glomerulonephritis, while the presence of proteinuria is generally indicative of some form of glomerular disease. The concurrent presence of hematuria with red cell casts, dysmorphic red cells, heavy proteinuria, or lipiduria can also help subclassify patients into those with an active "nephritic" sediment. This is commonly associated with AKI due to glomerulonephritis.

- Pyuria with white cell and granular or waxy casts and varying levels of proteinuria is suggestive of tubular or interstitial disease or urinary tract infection (see the picture below)

- White cells and white cell casts can also be seen in acute glomerulonephritis, particularly postinfectious glomerulonephritis. In this setting, however, there are also other signs of glomerular disease, such as hematuria, red cell casts, and proteinuria.

- Hematuria and pyuria with no or variable casts (excluding red cell casts) may be seen in acute interstitial nephritis, glomerular disease, vasculitis, obstruction, and renal infarction.

Urine sodium excretion - With AKI in children, measurement of the urine sodium concentration is helpful in distinguishing ATN from prerenal AKI due to effective volume depletion. The urine sodium concentration is usually above 30 to $40 \mathrm{mEq} / \mathrm{L}$ and below $10 \mathrm{mEq} / \mathrm{L}$ in the former and latter conditions, respectively. Since normal newborns have a relatively decreased ability to conserve sodium, prerenal disease is usually associated with somewhat increased urine sodium concentrations (less than 20 to $30 \mathrm{mEq} / \mathrm{L}$ ).

However, since the urinary sodium concentration is influenced by the urine output, there is substantial overlap between ATN and prerenal disease. As an example, a given rate of sodium 
excretion will be associated with a lower urine sodium concentration by dilution in patients who have a high urine output.

- A value below 1 percent suggests prerenal disease, where the reabsorption of almost all of the filtered sodium represents an appropriate response to decreased renal perfusion.

- A value between 1 and 2 percent may be seen with either disorder.

- A value above 2 percent usually indicates ATN.

- In newborns, prerenal disease and ATN are associated with FENa values of less than 2.5 percent and greater than 2.5 to 3.5 percent, respectively, because of their decreased ability to reabsorb sodium.

The FENa is most useful in patients with severe renal failure and low urine output (oliguria). It is less accurate in those with a normal or moderately reduced GFR because the value determining a prerenal state changes continuously with the GFR. FENa may also be elevated after the administration of either a distal or loop diuretic due to the increase in urine sodium excretion. Why this occurs is discussed in detail separately. A low FENa is not unique to prerenal disease, since it can occur in disorders associated with normal tubular function but a low GFR. These include acute glomerulonephritis, vasculitis, and acute urinary tract obstruction. It can also be seen when ATN is superimposed upon a chronic sodium-retaining state.

Urine osmolality - Loss of concentrating ability is an early and almost universal finding in ATN with the urine osmolality usually being below $350 \mathrm{mosmol} / \mathrm{kg}$. However, lower values similar to those in ATN may be seen in prerenal disease and are therefore of little diagnostic help. In contrast, a urine osmolality above $500 \mathrm{mosmol} / \mathrm{kg}$ is highly suggestive of prerenal disease.

Urine volume - The urine volume is typically, but not always, low (oliguria) in prerenal disease due to the combination of sodium and water avidity. In comparison, patients with ATN may be either oliguric or nonoliguric.

Response to volume repletion - Unless contraindicated, a child with a clinical history consistent with fluid loss (such as vomiting and diarrhea), a physical examination consistent with hypovolemia (hypotension and tachycardia), and/or oliguria should be administered intravenous fluid therapy. This fluid challenge attempts to identify prerenal failure that can progress to ATN if not treated promptly. However, such fluid infusion is contraindicated in those with obvious volume overload or heart failure.

Commonly used fluids are crystalloid solutions, such as normal saline $(20 \mathrm{~mL} / \mathrm{kg})$ administered over 20 to 30 minutes, which may be repeated. Restoration of adequate urine flow and improvement in renal function with fluid resuscitation is consistent with prerenal disease. However, if urine output does not increase and renal function fails to improve with the restoration of intravascular volume, invasive monitoring may be required to adequately assess the child's fluid status and help guide further therapy. 


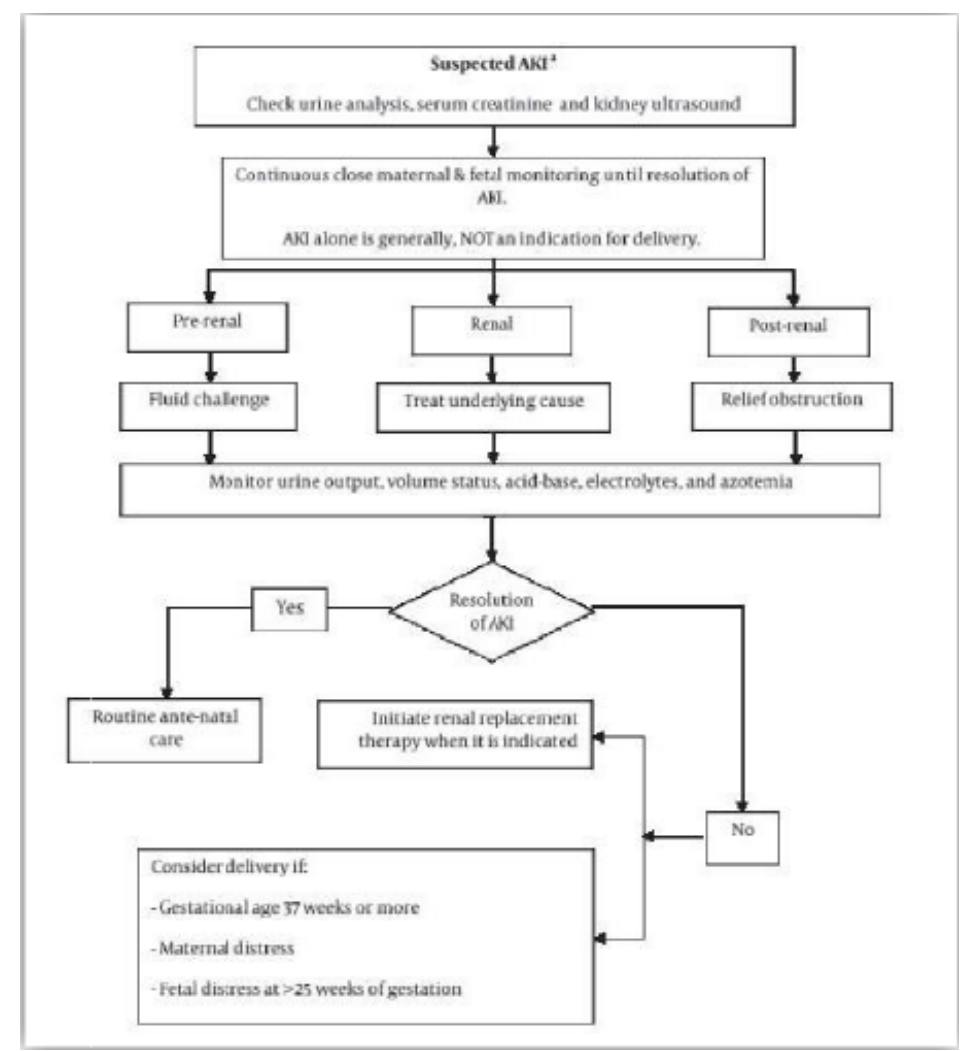

Fig 4; The diagnostic algorithm of AKI

\section{Additional laboratory measurements}

Complete blood count - Severe microangiopathic hemolytic anemia associated with thrombocytopenia in the setting of AKI confirms the diagnosis of HUS. . Severe hemolysis, whether drug-induced or secondary to hemoglobinopathies, may also result in ATN due to massive hemoglobinuria.

Other abnormalities - The measurement of additional blood components may be diagnostically helpful in certain settings. As examples:

- In children with a clinical picture consistent with rapidly progressive glomerulonephritis (RPGN), the presence of anti-neutrophil cytoplasmic antibodies (ANCA), anti-nuclear antibodies (ANA), anti-glomerular basement membrane (GBM) antibodies, antistreptococcal antibodies, and/or hypocomplementemia is associated with certain inflammatory disorders (24, 25).

- Eosinophilia and/or urine eosinophiluria may be present in some cases of interstitial nephritis.

- Markedly elevated uric acid levels may also induce AKI. Thus, tumor lysis syndrome secondary to chemotherapy treatment of childhood leukemia or lymphoma may result in AKI due to urate nephropathy (26).

- Although not diagnostically helpful, hyperkalemia, hyperphosphatemia, hypocalcemia, and acidosis can be observed in AKI:

- Hyperkalemia. The ability to maintain potassium excretion at near normal levels is generally maintained in patients with renal disease as long as both aldosterone secretion and distal flow are maintained. Thus, hyperkalemia generally develops in the patient who is oliguric or who has an additional problem, such as a high potassium diet and increased tissue breakdown.

- Hyperphosphatemia. Once the GFR falls below threshold levels, the renal excretion of phosphorus decreases, resulting in hyperphosphatemia. 
Texila International Journal of Clinical Research

Volume 3, Issue 2, Dec 2016

- Hypocalcemia. Hypocalcemia can result from hyperphosphatemia, decreased calcium absorption in the gastrointestinal tract (due to inadequate renal production 1, 25-vitamin D), and/or skeletal resistance to parathyroid hormone (PTH).

- Acid-base balance is normally maintained by the renal excretion of the daily acid load (about 1 $\mathrm{mEq} / \mathrm{kg}$ per day, derived mostly from the generation of sulfuric acid during the metabolism of sulfur-containing amino acids). Elimination of this acid load is achieved by the urinary excretion of hydrogen ions. A metabolic acidosis may therefore ensue with AKI.

Renal imaging - Renal ultrasonography should be performed in all children with AKI of unclear etiology. It can document the presence of one or two kidneys, delineate renal size, and help survey renal parenchyma (27). It is particularly useful in diagnosing urinary tract obstruction or occlusion of the major renal vessels.

Renal biopsy - A renal biopsy is most commonly obtained when noninvasive evaluation has been unable to establish the correct diagnosis (28).

Biomarkers - Biomarkers have been identified that may identify children in the beginning stages of AKI. In one study of 71 children and infants who underwent cardiopulmonary bypass surgery, urinary levels of neutrophil gelatinase-associated lipocalin (NGAL) were found to be elevated 2 hours after bypass in children who subsequently developed AKI. In addition to NGAL, interlukin-18 has been reported as an early marker of AKI in children (29, 30).

RIFLE Criteria - The Acute Dialysis Quality Initiative has developed criteria to standardize the reporting and classification of AKI in adults. Five levels of AKI are based upon degree of elevation of serum creatinine, urine output, and requirement for dialysis. In adults, the RIFLE criteria have been shown to predict mortality, renal outcome, and length and cost of hospital stay. Both prospective and retrospective studies have shown that renal injury occurs in a significant number of children admitted to the pediatric intensive care unit (PICU) when the RIFLE criteria are used to assess renal function (31). This was illustrated in a retrospective study of 3396 admissions to a single PICU between 2003 and 2006 (32). Six percent of patients $(n=194)$ had AKI on admission, and 10 percent of patients (n =339) developed AKI during their PICU stay based upon RIFLE criteria. Based on regression analysis, mortality increased as the severity of AKI increased based upon RIFLE score. Length of PICU stay was also increased in patients with AKI compared to those without AKI.

Standardized classification criteria, such as the RIFLE criteria, can be used as research tools to improve the understanding of AKI epidemiology and potentially identify treatable risk factors.

\section{Treatment of AKI}

Measures to correct underlying causes of acute kidney injury (AKI) should begin at the earliest indication of renal dysfunction. Serum creatinine does not rise to abnormal levels until a large proportion of the renal mass is damaged, because the relationship between the glomerular filtration rate (GFR) and the serum creatinine level is not linear, especially early in disease. Indeed, the rise of serum creatinine may not be evident before $50 \%$ of the GFR is lost.

It cannot be overstated that the current treatment for AKI is mainly supportive in nature; no therapeutic modalities to date have shown efficacy in treating the condition. Therapeutic agents (eg, dopamine, nesiritide, fenoldopam, mannitol) are not indicated in the management of AKI and may be harmful for the patient.

Maintenance of volume homeostasis and correction of biochemical abnormalities remain the primary goals of treatment and may include the following measures:

- Correction of fluid overload with furosemide

- Correction of severe acidosis with bicarbonate administration, which can be important as a bridge to dialysis

- Correction of hyperkalemia

- Correction of hematologic abnormalities (eg, anemia, uremic platelet dysfunction) with measures such as transfusions and administration of desmopressin or estrogens 


\section{Volume overload}

Furosemide can be used to correct volume overload when patients are still responsive; this often requires high intravenous (IV) doses. Furosemide plays no role in converting an oliguric AKI to a nonoliguric AKI or in increasing urine output when a patient is not hypervolemic. However, response to furosemide can be taken as a good prognostic sign.

\section{Hyperkalemia}

Hyperkalemia in patients with AKI can be life-threatening. Approaches to lowering serum potassium include the following:

- Decreasing the intake of potassium in diet or tube feeds

- Exchanging potassium across the gut lumen using potassium-binding resins

- Promoting intracellular shifts in potassium with insulin, dextrose solutions, and beta agonists

- Instituting dialysis

\section{Nephrotoxic agents}

In AKI, the kidneys are especially vulnerable to the toxic effects of various chemicals. All nephrotoxic agents (eg, radiocontrast agents, antibiotics with nephrotoxic potential, heavy metal preparations, cancer chemotherapeutic agents, nonsteroidal anti-inflammatory drugs [NSAIDs]) should be avoided or used with extreme caution. Similarly, all medications cleared by renal excretion should be avoided, or their doses should be adjusted appropriately.

A 2013 study indicated that triple therapy using nonsteroidal anti-inflammatory drugs (NSAIDs) with 2 antihypertensive medications - a diuretic along with an angiotensin-converting enzyme (ACE) inhibitor or an angiotensin-receptor blocker (ARB) — significantly increases the risk of hospitalization for AKI, particularly in the first 30 days of treatment with these drugs.

\section{Summary}

Acute kidney injury (formerly known as acute renal failure) is a syndrome characterised by the rapid loss of the kidney's excretory function and is typically diagnosed by the accumulation of end products of nitrogen metabolism (urea and creatinine) or decreased urine output, or both. It is the clinical manifestation of several disorders that affect the kidney acutely. Acute kidney injury is common in hospital patients and very common in critically ill patients. In these patients, it is most often secondary to extrarenal events. How such events cause acute kidney injury is controversial. No specific therapies have emerged that can attenuate acute kidney injury or expedite recovery; thus, treatment is supportive. New diagnostic techniques (eg, renal biomarkers) might help with early diagnosis. Patients are given renal replacement therapy if acute kidney injury is severe and biochemical or volume-related, or if uraemic-toxaemia-related complications are of concern. If patients survive their illness and do not have premorbid chronic kidney disease, they typically recover to dialysis independence. However, evidence suggests that patients who have had acute kidney injury are at increased risk of subsequent chronic kidney disease.

\section{References}

[1] Andreoli SP. Acute renal failure. CurrOpinPediatr 2002; 14:183.

[2] Akcan-Arikan A, Zappitelli M, Loftis LL, et al. Modified RIFLE criteria in critically ill children with acute kidney injury. Kidney Int 2007; 71:1028.

[3] Chan JC, Williams DM, Roth KS. Kidney failure in infants and children. Pediatr Rev 2002; 23:47.

[4] Chertow GM, Levy EM, Hammermeister KE, Grover F, Daley J:Independent association between acute renal failure and mortality following cardiac surgery. JAMA1998, 104:343-348).

[5] de Mendonca A, Vincent JL, Suter PM, Moreno R, Dearden NM, Antonelli M, Takala J, Sprung C, Cantraine F: Acute renal failure in the ICU: risk factors and outcome evaluation by SOFA score. Intensive Care Med2000, 26:915-921). 
Texila International Journal of Clinical Research

Volume 3, Issue 2, Dec 2016

[6] Flynn JT. Choice of dialysis modality for management of pediatric acute renal failure. PediatrNephrol 2002; 17:61.

[7] Hui-Stickle S, Brewer ED, Goldstein SL. Pediatric ARF epidemiology at a tertiary care center from 1999 to 2001. Am J Kidney Dis 2005; 45:96.

[8] Hui-Stickle S, Brewer ED, Goldstein SL. Pediatric ARF epidemiology at a tertiary care center from 1999 to 2001. Am J Kidney Dis 2005; 45:96.

[9] http://www.webmd.com/a-to-z-guides/understanding-kidney-disease-.basic-information

[10] Hiraoka M, Hori C, Tsuchida S, et al. Ultrasonographic findings of acute tubulointerstitial nephritis. Am J Nephrol 1996; 16:154.

[11] Hoschek JC, Dreyer P, Dahal S, Walker PD. Rapidly progressive renal failure in childhood. Am J Kidney Dis 2002; 40:1342.

[12] http://emedicine.medscape.com/article/243492-overview\#a1

[13] Harrison's Principles of Internal Medicine, 17th ed, Braunwald E, Fauci AS, Kasper DL, et al (Eds), McGraw Hill Book Company. New York 2008.

[14] Kist-van Holthe tot Echten JE, Goedvolk CA, Doornaar MB, et al. Acute renal insufficiency and renal replacement therapy after pediatric cardiopulmonary bypass surgery. PediatrCardiol 2001; 22:321.

[15] Kist-van Holthe tot Echten JE, Goedvolk CA, Doornaar MB, et al. Acute renal insufficiency and renal replacement therapy after pediatric cardiopulmonary bypass surgery. PediatrCardiol 2001; 22:321)

[16] Kidney Disease: Improving Global Outcomes. Clinical practice guideline on acute kidney injury. 2011. www.kdigo.org.

[17] (Kidney Disease: Improving Global Outcomes (KDIGO) Acute Kidney Injury Work Group. KDIGO Clinical Practice Guideline for Acute Kidney Injury. Kidney Int. 2012;2:124-138

[18]

[19] Lee WS, Ooi TL. Deaths following acute diarrhoeal diseases among hospitalised infants in Kuala Lumpur. Med J Malaysia 1999; 54:303.

[20] Lee WS, Ooi TL. Deaths following acute diarrhoeal diseases among hospitalised infants in Kuala Lumpur. Med J Malaysia 1999; 54:303.

[21] Mishra J, Dent C, Tarabishi R, et al. Neutrophil gelatinase-associated lipocalin (NGAL) as a biomarker for acute renal injury after cardiac surgery. Lancet 2005; 365:1231.

[22] Moffett BS, Goldstein SL. Acute kidney injury and increasing nephrotoxic-medication exposure in noncritically-ill children. Clin J Am SocNephrol 2011; 6:856.

[23] Mehta RL, Kellum JA, Shah SV, et al. 2007. Acute Kidney Injury Network (AKIN): report of an initiative to improve outcomes in acute kidney injury. Critical care, 11, R 31

[24] Molitoris BA, Levin A, Warnock D, et al. Improving outcomes of acute kidney injury: report of an initiative. Nat ClinPractNephrol 2007; 3 (8): 439-44

[25] Moghal NE, Brocklebank JT, Meadow SR. A review of acute renal failure in children: incidence, etiology and outcome. ClinNephrol 1998; 49:91

[26] Mehta RL, Kellum JA, Shah SV, Molitoris BA, Ronco C, Warnock DG, et al. Acute Kidney Injury Network: report of an initiative to improve outcomes in acute kidney injury. Crit Care. 2007. 11(2):R31

[27] Moghal NE, Brocklebank JT, Meadow SR. A review of acute renal failure in children: incidence, etiology and outcome. ClinNephrol 1998; 49:91.].

[28] Rossi R, Kleta R, Ehrich JH. Renal involvement in children with malignancies. PediatrNephrol 1999; 13:153.

[29] Schrier RW, Wang W, Poole B, Mitra A. Acute renal failure: definitions, diagnosis, pathogenesis, and therapy. J Clin Invest. 2004 Jul. 114(1):5-14.

[30] SVachvanichsanong P, Dissaneewate P, Lim A, McNeil E. Childhood acute renal failure: 22-year experience in a university hospital in southern Thailand. Pediatrics 2006; 118:e786

[31] Schneider J, Khemani R, Grushkin C, Bart R. Serum creatinine as stratified in the RIFLE score for acute kidney injury is associated with mortality and length of stay for children in the pediatric intensive care unit. Crit Care Med 2010; 38:933. 
Texila International Journal of Clinical Research

Volume 3, Issue 2, Dec 2016

[32] The Harriet Lane Handbook, 15th, Siberry GK, Iannone R (Eds), Mosby, St. Louis 2000)

[33] Washburn KK, Zappitelli M, Arikan AA, et al. Urinary interleukin-18 is an acute kidney injury biomarker in critically ill children. Nephrol Dial Transplant 2008; 23:566 


\title{
Counselling and Moral Education: A Panacea in Curbing the Increasing Trends of Agitation in Nigeria
}

\author{
Article by Sr. Victoria Uzoamaka Ezeh \\ Ph.D in Guidance and Counseling Psychology, Texila American University, Nigeria \\ Email: sistervictoriaeze@texilaconnect.com
}

\begin{abstract}
Education has been the bedrock of national development since inception but in recent times Nigeria has witnessed a lot of agitations in nearly all the geo-political zone of the federation which has stagnated national unity. This increasing trend of agitations has taken another shape especially in the Niger delta region and other parts of the country which has left political unrest, social, economic and psychological losses and pains. This paper therefore explored frustration aggression theory and consent that aggression is always based on frustration which is the justification for the increasing trends of agitations in Nigeria. Also this paper, examined the different group of agitators based on their demand and then focuses on counselling and moral education which is a universal permanent and pervasive phenomenon in human society as well as the earliest factors that influence human life as a panacea in curbing the increasing trends of agitations in Nigeria. The concept of counselling and moral education was explained and recommendation was also given.
\end{abstract}

Keywords: moral education, counselling, agitation, youth, Nigeria and violence.

\section{Introduction}

Nigeria is a plural and heterogeneous state with over 350 ethnic groups with the Hausa-Fulani, Yoruba and Igbos making the majority tribes (The New York Times, 2012). This explains the Nigerian conundrum though with a federal structure which was until 1914 a diffusedly independent groups which occasionally relates with one another is a peripheral state with over 400 languages (Egbe, 2004) and about 190 million people, resource allocation has always been centralized thereby causing frustration, aggression and hunger for the control of the centre. A society, made up with diverse belief systems, customs and institutions where violence is inevitable as a result of conscious opposition to one or more groups because these groups or persons appear to have incompatible interest. Since independence in 1960, the country has witnessed a lot of violent eruption, ethno religious crisis, the Biafra war and general insecurity in nearly all the state of the federation. But more recently, the sporadic agitations in nearly all the geo-political zone of the federation have resulted in the operation of several militia groups. Notable among these groups are the Niger Delta Avengers, Boko Haram and Indigenous people of Biafra among others. The operation of this militia groups constituted by large number of youth has coursed a lot of havoc in the country leading to social and economic dislocation, psychological losses, destruction of property, kidnapping and all order forms of maladies.

Globally youth are faced with enormous problems. Atwater (1988) argues that the adolescent stage of life is marked by emotional development that includes mood swings, enthusiasm, tenderness, cruelty, curiosity and apathy. This however explains why adolescents out of tension, frustration and aggression often indulge in violent act which if not checkmate could leads to formation of group agitations. This adolescents are not matured enough to critically evaluate baffling situations they continuously faced which means they need somebody to help them in the solution of problems and thus avoid tensions, frustration and conflicts. The youth must know their capabilities and potentialities, especially in areas that are crucial for the realisation of objectives in life. Their goals, aspirations and expectations are to be shaped accordingly. Corey (1991) holds that counselling is a 
service designed to help an individual to analyze himself/herself by relating his capabilities, achievements, interests and mode of adjustment. As explained by Biswalo (1996), the need for counselling services today could be due to the ever- growing complexity of the society and people have to learn how to cope with the upcoming challenges. Morally, adolescent need to be taught the right conducts, not only in their immediate social relations, but also in their dealings with their fellow citizens and with the whole of human race. Counselling services and moral education are imbedded in every educational system but lack of adequate attention by the government over the years has exposed these adolescent to society where violence and agitations is the only way to dispose their demand and grievance. Consequently, several strategies and measures have been adopted by the federal government which includes dialogue, amnesty and military interventions over the years in response to the request of this militia groups has yielded insignificant results. Therefore it is against this background that this paper suggests the use of counselling and moral education as a panacea in curbing the increasing trends of agitations in Nigeria.

\section{Conceptual and theoretical explanation}

\section{Counselling}

Counselling is a learning process in which a counsellor helps an individual or individuals learn, understand themselves and their environment and be in a position to choose the right type of behaviours that will help them develop, grow, progress, ascend, mature and step up, educationally, vocationally and socio personally (Egbo, 2013). Mutie and Ndambuki (2004) assert that counselling service is the brain and heart of the guidance programme. Thus counselling represents a part of the total process of guidance which is helping individuals, achieve the self- understanding and selfdirection necessary to make the maximum adjustment in a particular environment. Corey (1988) regards counselling as a process which occurs in one to one relationship between an individual troubled by problems with which he cannot cope with and a professional worker whose training and experiences have qualified him to help others reach solution to personal needs. Okoye (1990) viewed counselling as an interactional relationship designed to facilitate the personal development of information leading to effective decision making and awareness of the self.

\section{Moral education}

Etymologically, the word moral comes from a Latin word "mos or moris meaning the code or customs of a people, the social glue that defined how individuals should live together. Moral education is not a new idea; it is as old as society and education. It addresses ethical dimensions of the individual and society and examines how standard of right and wrong are developed. According to New Encyclopaedia Britannica Vol. 6, "Moral education is a designed moral value that guides people and in learning them towards their eventual role in the socieff. While Vol. 8 of the New Encyclopaedia Britannica define moral education as the development of sense of moral sensitivity and duty towards people and state. Fafunwa (1976) defined moral education as the inculcation of desirable values, norms, attitudes approved by the society in order to have good citizens in the future. In the light of the above definition, moral education is the key to the general sustenance of national integration and social orderliness. Moral education teaches core moral values, such as honesty and responsibility, care, etc and helps to raise morally responsible and self-disciplined citizens. Problem solving, decision-making and conflict resolution are also important parts of developing good moral character. Thus, to educate a person in mind and not in morals is to educate a menace in society (Omoregbe, 2000).

\section{Frustration aggression theory}

The frustration-aggression theory is associated with works of John Dollard et al (1939), the core assumption of which is that "aggression is based on frustration and this frustration always leads to aggression" (1939). Dollard et al. (1939) posited "that the occurrence of aggressive behaviour always 
presupposes the existence of frustration and, contrariwise, that the existence of frustration always leads to some form of aggression”. This theory presents the idea of relative deprivation as a perceived disparity between value expectation and value capabilities. Or the lack of a need satisfaction defined as a gap between aspiration and achievement (MidIarsky, 1975). Simply put, when there is a gap between the level of value expectation and the level of value attainment, due to lack of capability to establish a congruence between both levels, tension builds up due to the pressure of an unfulfilled aspiration or an unsatisfied urge or need. This, when not arrested in time, leads to frustration. Frustration, when it builds up, leads to the rising up of suppressed emotions of anger, which is often directed against the party considered to be the source of deprivation of satisfaction. This strong emotion finally finds an outlet through aggressive and invariably violent disposition towards the environment. The increasing trends of agitations by several militia groups against the government in Nigeria are viewed in this perspective. This however, is perceived to be of sufficient generality to provide a basis for the explanation of virtually all forms of agitations and aggressive behaviour which is the appropriate description of the increasing trends of agitations in Nigeria.

\section{Trend of agitations in geo political zone of nigeria}

Over the years there have been several militia groups agitating for one course or the other in all geo-political zone of the federation. But for the focus of this paper, the more recent trend of militia group from the south-south, south-east and north-east shall be considered.

\section{South-South}

The Niger Delta is one of the largest deltas in the world, probably the third largest on earth. The region is regarded as one of the nine most difficult deltas of the world comparable to the Mekong, the Amazon and the Ganges. It is situated in the central part of southern Nigeria. It lies within latitudes 4 degrees north to 6 degrees north, and longitude 5 degrees east to 8 degrees east (Azaiki, 2007: 1; Ile \& Akukwe, 2001). The area has a population of about twenty five million people, according to the 2006 national census figures (National Bureau of Statistics, 2006) and more than forty ethnic groups with links to the linguistic groups of Ijaw, Edo, Igbo, Efik, Ibibio and Oron. "It is rich in both renewable and non renewable natural resources such as oil, gas, bitumen, etc, accounting for $95 \%$ of the total revenue for the Nigerian government, generated from oil and gas exploration and $80 \%$ of national wealth” (Brisibie, 2001: 1; Tell, 2008: 33).

The combination of the activities of the oil companies and the government led to emergence of various armed groups operating under such names as Egbesu Boys, Movement for the Survival of the Ijaw Ethnic Nationality (MOSEIN), Joint Revolutionary Council (JRC), Arogbo Freedom fighters, Niger Delta Volunteer Force, the Movement for the Emancipation of the Niger Delta (MEND) and Niger Delta Avengers (NDA) among others. Their youths had drawn both local and international attention to their deplorable situation through their restiveness. This explains the attack on oil pipelines in the region which has created instability and has eating deep into the wealth of the nation. Their agitation is primarily based on quest for resource control as a result of oil discovery in the region while the violence in Niger Delta has its roots in the increasing protest of the region against their political, economic and environmental disenfranchisement (Kelly, 2008).

\section{North-East}

The emergence of Boko Haram is in the north-east is traceable to the blistering preaching of Mallam Abubakar Kilakam an indigene of Niger Republic resident in Maiduguri, Borno state. He propagated the anti- government message that led to his arrest and eventually deported to Niger Republic. The idea (Boko Haram) activity has been operating covertly for several years even before the emergence of late Mohammed Yusuf. Who it seems only met a fertile ground and capitalized on it to spread his gospel. Boko Haram sect derives its message largely from the ideology of one of the world terrorist groups Al-qaida (Omipidan, 2013). The philosophy of the sect is rooted in the practice of orthodox Islam. Orthodox Islam in their interpretation abhors Western education and the civil 
service bureaucratic system. This explains why the sect is popularly known as the Boko Haram, literally meaning 'Western education is a sin' (Boyle, 2010). The agitation by Boko Haram for the imposition of Shariah Penal code in the 36 states of the federation is primitive and unconstitutional as Nigeria is a secular state. The sect strongly advocate for the abrogation of any form of western education and culture generally and it draws its members mainly from disaffected youths and unemployed graduates, including former Almajiris (Street Children) who migrated from the rural areas to urban areas in search of better means of livelihood or to study under renowned Islamic teachers in cities like Kano, Zaria, Kaduna, and Maiduguri. The sect also has some well educated, wealthy and influential people as members. The sect is estimated to have over 280000 members across the 19 states of northern Nigeria, Niger Republic, Chad and Sudan. There demand has led to several activities like kidnappings, bombings and suicide attacks at both government and religious institutions that parade a huge number of people at a time. Since its military operation in 2009, about 17,000 people have been killed especially in the north-eastern Nigeria (Qaqar, 2012).

\section{South-East}

Since after the Biafra war, several groups have emerged in the south-east regions which are Movement for the Actualization of the Sovereign State of Biafra (MASSOB), Biafra Zionist Movement (BZM) and Indigenous people of Biafra (IPOB) among others. Generally the Igbos have craved for a just society where every ethnic groups can compete favourably for political power and where economic resources is equitably distributed through non- violence. In 1999, Ralph Uwazuruike formed a militia group called MASSOB which embarked on several demonstrations and also established a radio station in the US for the purposes of reaching out to Igbos in the Diasporas and attracting international attentions (TELL, 2004 in Ojukwu, 2009b). In 2012, another group called the BZM founded by a United Kingdom- based lawyer, Benjamin Onwuka claimed that an application has been sent to the UN for an observer status for the Republic of Biafra on the ground that the security of lives and property, practice of religion and freedom of Association of the Biafra People are no longer guaranteed in the entity called Nigeria, hence the call for an independent state on 5th November 2012 (Uzodima, 2014). Presently in south-east this recent agitations are from the IPOB led by Nnamdi Kanu, a British- Nigerian. Their claims are that the present administration have hounded and short-changed the Igbos in the scheme of things causing disharmony hence the call for a divided Nigeria. This is informed by the incessant hardship, lack of holistic development in the socioeconomic landscape of Nigeria, lack of youth employment, corruption in high offices and economic regression. Though it claimed to have existed since 2012 by a group of people from the South-South and South-east regions of the country in London, nothing was heard from them until the inauguration of President Buhari.

\section{Counselling and moral education as a panacea in addressing the increasing trends of agitations in nigeria}

In Nigerian Philosophy of Education, it is believed that education is a pivot for national development and all round development of an individual. In this end, the formulation of ideas, their integration for National development and the interaction of persons and ideas are all aspects of education (Federal Republic of Nigeria 2004). The role of education in the development of an individual and the society at large cannot be overemphasized. Education is not only a veritable tool for the transmission of norms and values to the younger generations but also a tool for liberation from ignorance and high rate of docility. When teaching and learning improves, social vices shall reduce and the communities and the larger society shall enjoy peaceful co-existence whose values cannot be overemphasized.

Counselling and moral education has always been a perennial aim of education that enables the students in enriching their perspective and promoting attitude towards resolving educational, vocational and personal problems. Fuster (2002) opined that the goals of counselling as facilitating behaviour change, enhancing coping skills, promoting decision-making, improving relationships and 
facilitating the client's potential. It is a personalized and individualised process for helping the individual to learn and acquire habits, skills, attitudes and interests which make him a normally adjusted being. Therefore, it is evident that the process and goals of counselling is to help the student move towards a greater level of self understanding.

The need for counselling and moral education in modern times has increased because of the multiplicity of problems that the individuals have to face in the various domains of life. The adolescent children may then inadvertently and unconsciously respond with the problems. They may feel disempowered and overwhelmed by the current system of education. In this rapidly moving complex society, the students are struggling for the better adjustment and existence. If they are not getting proper guidance they may engage in anti-social activities. Therefore it becomes pertinent that they catch them young because the age of the students during adolescence is sensitive and highly inflammable.

Counselling and moral education is not a quality which human beings acquire automatically, it entails directing human energies toward constructive social goals which has to be instilled by training and education so that a set of values may be formed. Thus the whole person is prepared for participation in the moral institutions with a proper sense of conscience, polity, law and economy. It implants in each individual such dispositions as shall enable them to live and work harmoniously with others, both in the present school premises and for the future, and on every level of human interaction (Charles 1963).

Counselling and moral education is a very important and inevitable tool which could avert the condition of disharmony in society. Moral education is universal and essential to every human culture. As we all know that any society without a modicum of morality must collapse. In this sense, morality is the observance of rules for the harmonious adjustment of the interests of the individual to those of others in society (Wiredu 1992). According to Whiteley (1982), morality is social in a more fundamental sense, as an outline of norms of behaviour operative in a community. To this end, life in a human community is possible, only if there is a certain level of friendliness, mutual aid and rules for settling issues. Without these facilities, a society cannot survive or exist at all.

\section{Conclusion}

There is an increasing trend of agitations in nearly all the geo-political zone of the federation which have led to the operation of several militia groups converting our youth into hooligans, terrorists, oil bunkering, hijackers, vandals etc across regions and boundaries of the federation. It is obvious that the shortfalls of students' education which has made them hostile in the society are seen mainly in the inability of the various governments and educational sectors to consistently and institutionally moderate the conduct of the youths in view of civilized conduct. To this end, the problem of agitations could be seen as lack of counselling and moral education on the part of the youth. Therefore there is a striking and urgent need for government to consider counselling and moral education as a panacea if the increasing trend of agitations must be curbed.

\section{Recommendations}

The following are the recommended in curbing the increasing trend of agitations in Nigeria.

- Government should make counselling and moral education an integral part of the education process and that more time and status should be allocated.

- Government should support counselling and moral education practically by providing and making funds available.

- Parents also should be included in guidance and counselling programme through giving them progressive report of their children.

- The inculcation of moral value should not rely on the government alone; parents, teachers, religious leaders and community leaders are expected to contribute to the trend. 
Texila International Journal of Clinical Research

Volume 3, Issue 2, Dec 2016

- Youths should learn to identify pride in self-worth regardless of race, colour, or creed, discrimination will disappear from society when persons are judged not by skin colour, ethnic groups, or the class to which they belong, but by their ability and integrity.

- Government should provide the right education and training that will ensure the possession of a stable skill suitable for youth to enhance youth participation in social orderliness and national development.

\section{References}

[1] Atwater, E. (1988). Adolescence. (2nd Ed). New Jersey: Prentice Hall, Inc.

[2] Azaiki, S. (2007). Inequities in Nigerian Politics. Ibadan: Y-Books.

[3] Biswalo, (1996). An introduction to guidance and Counselling Diverse Africa Contexts. Dare Salaam: Dare salaam University Press.

[4] Boyle, P. (2010). 'Nigeria's Taliban Enigma'. BBC News, 31 July 2009, http://news.bbc.co.uk/2/hi/8172270.stm (accessed 8 July 2010).

[5] Charles, E. C. (1963). Moral Education in family, school and church, (Philadelphia: The West Minster Press, p.60. 2.

[6] Corey G. (1988). Issues and Ethics in the Helping professions. Pacific Grove, CA, Brooks Cole.

[7] Corey, G. (1991). Theory and Practice of Counselling and Psychotherapy. 4th ed. Pacific Grove. California: Brooks/Cole Publishing Company.

[8] Dollard, J. Miller, N. E, Doob, L.W, Mowrer, O.H, Sears, R.(1939). Frustration and Aggression. New Haven, Yale University Press.

[9] Egbe, M. E. (2004). The State and Civil Society in Nigeria: Study of the Movement for the Survival for the Ogoni People (MOSOP) Lagos: NIIA Occasional Papers.

[10] Egbo, A.C. (2013). Development of Guidance and counselling, Enugu; Joe best publishers.

[11] Fafunwa, B. (1976). History of Education in Nigeria. London: George Allen and Unwin Ltd.

[12] Federal Ministry of Education (2004). National policy on Education, Lagos federal Government printers.

[13] Ile, C. \& Akukwe, C. (2001). Niger delta, Nigeria, Issues, Challenges and opportunities for equitable development. Retrieved on 27/10/2011 from http://nigeriaworld.com/feature/article/niger-delta.html

[14] Kelly (2008). Bringing peace to the Niger Delta. www.usip.org/pubs/unpeacebriefings_niger_delta html.

[15] Midlarsky, M. (1975). On War: Political Violence in the International System, New York, Free Press.

[16] Mutie, E. K. \& Ndambuki, P. (2004). Guidance and Counselling for Schools and Colleges. Nairobi: Oxford University Press.

[17] National Bureau of Statistics (2006). Federal Republic of Nigeria 2006 Population Census. accessed 26/10/2011 from http://www.nigerianstat.gov.ng/nbsapps/Connections/Pop2006.pdf

[18] Nigeria Dangerous Slide: The New York Times 2012 (Retrieved 0n the 20 April 2013)

[19] Ojukwu C. C. (2009). Igbo Man’s Burden Abeokuta: EMI Publications.

[20] Okoye, A.U. (2010) Counselling in the industrial setting Visa Vis industrial relation. Aroka; Erudite publishers.

[21] Omipidan, I. (2013). Total War. www.sunnewspaper online 19 May 2013 (Retrieved on 22 May 2013

[22] Omoregbe, J. (2000). Ethics: A Systematic and Historical Study. Lagos: Joja Educational Research and Publishers.

[23] Uzodima, E. (2014). Biafra Zionists Federation attempts to seize Enugu radio station, kills police officer. Daily post 5 June.

[24] Whitely, C. H. (1982). The Justification of Moral Philosophy. Vol. 57, No. 222

[25] Wiredu, K. (1992). The Moral Foundations of an African Culture: In Kwasi Wiredu and Kwame Gyekye (eds) Person and Community Ghanaian Philosophical Studies 1. Washington D.C: The Council for Research in Values and Philosophy. 


\title{
The State of Mental Health in South Africa Cross Examination of the Vicious Cycle of the Demographic and Socio-Economic Factors that Affect Mental Health
}

\author{
Article by Sakibu Lyaga \\ M.Sc Counseling Psychology, Texila American University, Kenya \\ Email: Lsakibu@Yahoo.Com
}

\section{Introduction}

While numerous studies relate to mental illness, few provide prevalence estimates of diagnosable mental illness (e.g., major depressive disorder as opposed to feeling depressed, or generalized anxiety disorder as opposed to feeling anxious), and fewer still provide national prevalence estimates of diagnosable mental illness. It been proven that most people with alcohol or substance use and anxiety disorders experience them independently, but having both can be a vicious cycle. The symptoms of one disorder can make the symptoms another worse; an anxiety disorder may lead to using alcohol or other substances to self-medicate or alleviate anxiety symptoms. The co-occurrence of substance abuse, particularly alcohol abuse, is common among people who have social anxiety disorder. People with this disorder report that alcohol helps lessen their social anxiety, although it often makes it worse. Alcohol abuse usually develops after the onset of this disorder. PTSD and substance abuse commonly occur together. People suffering from this disorder often use alcohol or drugs to try to ease their anxiety, but substance abuse can exacerbate PTSD symptoms. Mental ill-health is strongly associated with poverty and social deprivation and living in poverty, exposure to stressful life events like crime and violence; inadequate housing, unemployment and social conflict, are all linked to mental ill-health. An overwhelming majority of people with mental and psychosocial disabilities are living in poverty, poor physical health, and are subject to human rights violations.

Mental health issues cannot be considered in isolation from other areas of development, such as education, employment, emergency responses and human rights capacity building. Despite overall global socio-economic development and improved social capital large part of this planet continues to be deprived. Poverty is one of the determinants of mental illnesses and social exclusion perpetuates deprivation. Since poverty is associated with exclusion, isolation, feelings of disempowerment, helplessness and hopelessness, it can lead to chronic insecurity and social mistrust, affecting people's mental well-being. Because of its close link to demographic and socio-economic factors, it is imperative that mental health is given as much weight or even more as a development issue and that appropriate programs are designed to address socioeconomic challenges resulting from mental disorders. In South Africa, as in many low- or middle-income countries (LMICs), the burden of mental disorders has grown over the past 20 years. Evidence suggests that nearly one in three South Africans will suffer from a mental disorder in his or her lifetime, a higher prevalence than many lowand middle-income countries. ( ).

Based on assessment with the Kessler Psychological Distress Scale, a measure of psychological distress, $17.1 \%$ of the patients (15.5\% of men and $19.4 \%$ of women) had severe psychological distress. Having no income, poor health status, migraine headache and tuberculosis as significant factors associated with severe psychological stress for men. For women the factors identified were lower education, no income, having been diagnosed with a sexually transmitted disease, stomach ulcer and migraine headache. Findings from World Health Organization World Mental Health surveys on the global burden of mental disorders have shown that at least one-third of all patients seen in primary care in LMICs present with common mental disorders (CMDs). Majority of these is not recognized or are ineffectively treated. Although depressive and anxiety disorders are classified as separate diagnostic categories in the ICD-10, 1 the concept of CMDs is valid for public health 
interventions owing to the high degree of co-morbidity between these disorders in primary care and the similarity in epidemiological profiles and treatment responsiveness.

In South Africa mental health is a vicious cycle whereby people with mental disorders will experience some of the worst forms of stigma and discrimination, linked to lack of awareness, misinformation and stereotyping about their condition. Consequently, they are denied the chance to participate fully in community activities or enjoy basic social services which pushes them further into poverty. Also the youths will start engaging in alcohol and substance abuse at very early ages which will reduce their chances of having a good education leading them to poverty and increasing their chances of developing mental disorders. Mental disorders negatively affect social capital of the affected and their families; yet this is a key asset for the poor and is an important element of sustainable poverty reduction. Some people with mental disorders become destructive, which often leads to strained relationships with neighbors and the need to spend money on dispute resolution at local courts; which further encroaches on their meager resources. Thus those who see their social capital eroded may face long-term, persistent and recurrent poverty. The mentally disturbed people will in most instances then are excluded from development programmes, membership of self help groups and government welfare schemes, and they find it difficult to find work. Even when they are willing to work, the rest of the community may be unwilling to offer them even casual work. There is the belief that mental illness is contagious exacerbates and intensifies stigma and exclusion.

The South African Stress and Health (SASH) 2009 described as the first large-scale populationbased study of common mental disorders in the country. This study was conducted between 2002 and 2004 and provides the only nationally representative data on the prevalence of common mental disorders. This was based on two international systems for the classification of mental disorders and other health problems, the ICD-10 and DSM-IV. These included anxiety disorders, such as panic disorder and post-traumatic stress disorder, mood disorders (a major depressive episode, for example), disorders to do with impulse control, as well as alcohol and drug disorders. The findings from the study showed that alcohol and substance abuse disorders were highly prevalent exacting a high emotional toll on individuals, families, and society further intensify the situation. Health24 gives a sobering overview of psychiatry in South Africa, they indicate that in South Africa it is estimated that between four-and-a-half to five million people are suffering from a psychiatric disorder. When alcohol and drug abuse are included in this figure, it rockets to a frightening 15 million people. Jonathan K Burns in 2010 summarizes the situation arguing by that "a significant 'mental health gap' between the major burden of mental and substance use disorders and the provision of psychiatric and mental health services”.

\section{General information about the lifetime prevalence in south africa}

In a study conducted in 2009 it was found that of $16 \%$ of the people suffering from mental disorders, only $25 \%$ had received treatment. (Inge, P., Sharon, K. et.al. 2009). Statistics from the South African Stress and Health further show that lifetime prevalence of common mental disorders was $30.3 \%$, and prevalence in the 12 months prior to the survey was $16.5 \%$ of the respondents identified as meeting criteria for a common mental disorder during the 12-months preceding assessment. One in every 4-5 people meets criteria for a mental disorder with severe impairment across their lifetime in all. The median age of onset was earlier for substance use disorders (21) than for anxiety disorders (32) or mood disorders (37). According to a study by Lund et al (2011) 75\% of people who live with a mental disorder in South Africa do not receive the care they need. SADAG put the figure at less than $16 \%$ of sufferers receive treatment for mental illnesses. Generally nearly threequarters of these sufferers are not accessing any form of mental health care at all. 


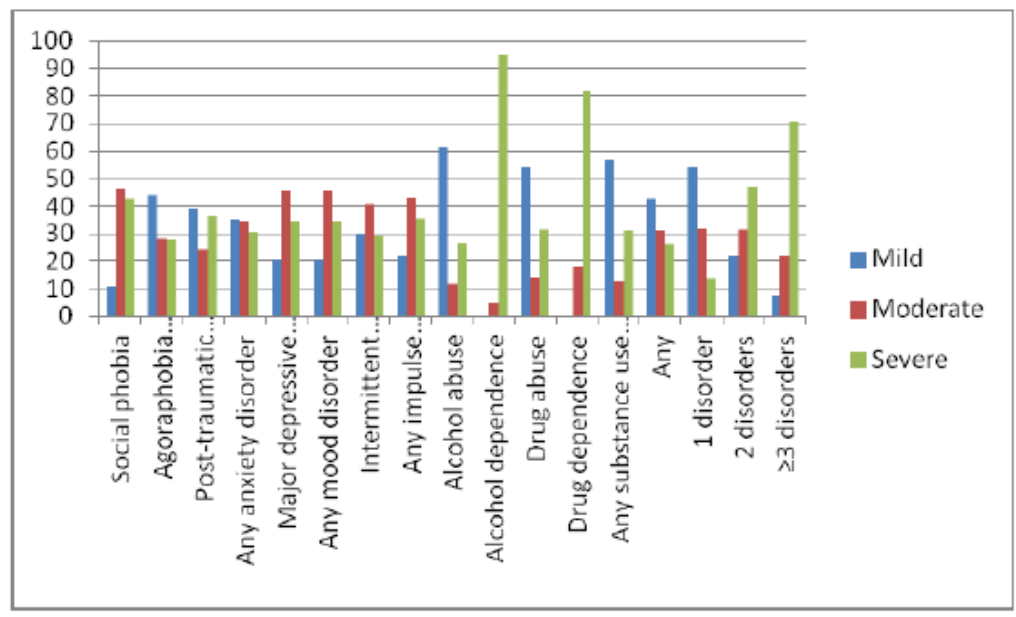

Fig 1

The research did find that $30.3 \%$ or around a third of the adult population would suffer from some form of mental disorder over the course of a lifetime with alcohol abuse (11.4\%) being the most common disorder. In fig 1 alcohol abuse dependence had the highest contribution to mental disorder prevalence followed by drug dependence and the chances of person suffering from a mental disorder suffering from more than three other disorders were highest compared to having one or two disorders. The mental health problems that most commonly co-occur with substance abuse were depression, anxiety disorders and bipolar disorder. In comparison to data from other countries, South Africa has a particularly high lifetime prevalence of substance use disorders. It has been shown that dealing with substance abuse, alcoholism or drug addiction problem is never easy and this may be even more difficult when one is dealing with mental health problems. When you have both a substance abuse problem and a mental health issue such as depression, bipolar disorder, or anxiety, it is called a cooccurring disorder or dual diagnosis.

According to statistics released by the South African Depression and Anxiety Group (SADAG) as many as one in six South Africans suffer from anxiety, depression or substance-use problems and this does not include more serious conditions such as bipolar disorder or schizophrenia). Dealing with substance abuse, alcoholism, or drug addiction is never easy, and it's even more difficult when one is also struggling with mental health problems. The ability of certain mental illnesses to exacerbate morbidity from several chronic diseases is well-established. Recent studies have explored the causal pathways from mental illness to certain chronic diseases, highlighting the need for more accurate and timely information on the epidemiology of mental illness. Co-morbidity is a two-way; every time someone is in a hospital bed being treated for one of the major health diseases such as heart disease or cancer that person may also have a mental health condition. Most of the time, those mental health concerns even it's just anxiety related to the actual treatment or chances of recovery from the disease are often overlooked altogether, or treated as minor, almost unrelated issues but there are treatments that can help. With proper treatment, support, and self-help strategies, one can overcome a dual diagnosis and reclaim their life.

\section{Understanding the link between substance abuse and mental health}

When it comes to substance abuse and mental health problems, the whole is greater than the sum of its parts. In the case of dual diagnosis or co-occurrence, both the mental health issue and the drug or alcohol addiction have their own unique symptoms that may get in the way of your ability to function, handle life's difficulties, and relate to others. To make the situation more complicated, the cooccurring disorders also affect each other and interact. When a mental health problem goes untreated, the substance abuse problem usually gets worse as well. And when alcohol or drug abuse increases, mental health problems usually increase too. Addiction is common in people with mental health 
Texila International Journal of Clinical Research

Volume 3, Issue 2, Dec 2016

problems. But although substance abuse and mental health disorders like depression and anxiety are closely linked, one does not directly cause the other.

Early experiences of adversity and stress have been associated with substance abuse, mental health problems and concurrent disorders which may put the youth at an even greater risk for concurrent disorders. These may include adverse childhood experiences such as experiences of abuse, neglect, and exposure to domestic violence are associated with substance abuse during the teen years (Dube, S.R., Anda, R.F. et.al. 2006). These early experiences are further carried on into adulthood resulting in adult mental health problems (Edwards, V. J., Anda, R. F. et.al. 2003). A growing body of research indicates that common protective factors can buffer the risks for both mental health problems and substance abuse. The protective factors include strong ties to family and school, supportive adults to talk to and feeling able to excel at something.

The British Columbia research argues that the presence of these protective factors is linked to fewer mental health and substance use problems and risky behaviour in those with both of these problems (McCreary Centre Society 2012). The most effective and efficient way to address a problem is to stop it before it starts, preventing concurrent disorders and their underlying problems means, at least in part, reducing the risk that accompanies early adversity and enhancing the benefits that result from common protective factors. Currently, most prevention efforts are developed for a specific health or behaviour problem. Therefore prevention efforts that address risk and protective factors can be effective at reducing substance abuse and produce a significant cost savings (Miller, T. \& Hendrie, D. 2008). The same is true for preventing mental health problems (Knapp, M., Parsonage, M. et.al. 2011).

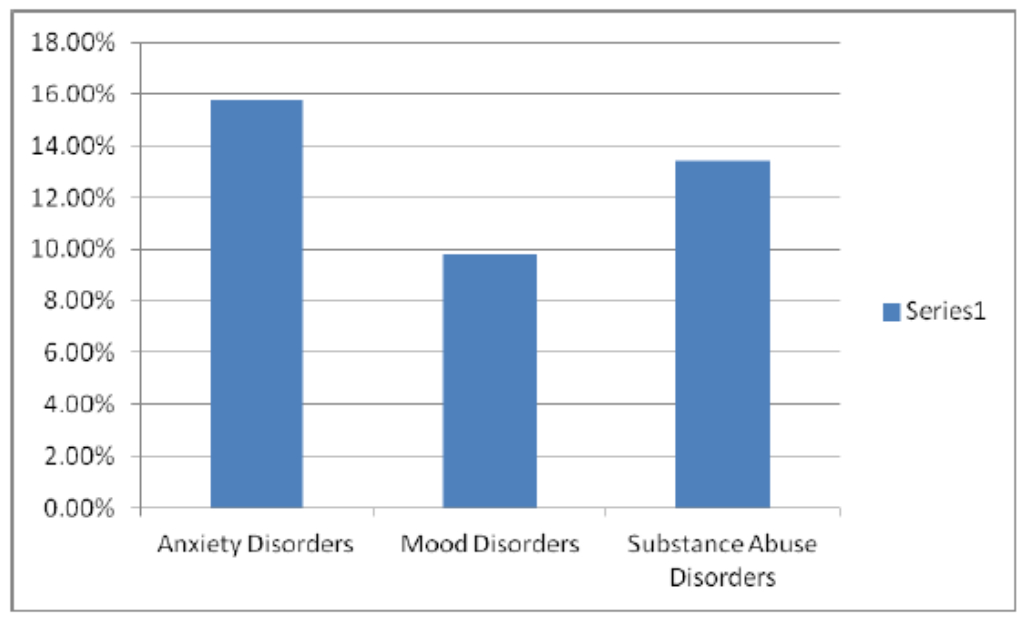

Fig 2

According to the lifetime prevalence of DSM-IV/CIDI disorders anxiety disorders had the highest prevalence (15.8\%), followed by substance use disorders (13.4\%), then mood disorders (9.8\%), and any disorder (30.3\%). Sunday Times indicated that more than 17 million people in South Africa are dealing with depression, substance abuse, anxiety, bipolar disorder and schizophrenia which accounts for around a third of South Africa's population of 51.8 million. Lifetime prevalence of substance abuse, but not other disorders, differed significantly across racial groups. Median age of onset was earlier for substance use disorders (21) than for anxiety disorders (32) or mood disorders (37). In comparison to data from other countries, South Africa has a particularly high lifetime prevalence of substance use disorders. These disorders have an early age of onset, providing an important target for the planning of local mental health services.

Some of the main reasons contributing to rampant substance abuse in South Africa is that many provinces are used as drug trafficking routes and as the South African government lacks the necessary resources to control this problem, many of these illicit drugs find their way into local populations as a 
drug. In addition, the availability of the weed plant allows for its rampant abuse among all age categories. It was found that $52 \%$ of street children smoke the Cannabis plant and $22 \%$ on a daily basis. Educational campaigns are limited, and as a result, many do not realize the impinging health effects that will result from substance abuse. There is evidence that alcohol or drugs are often used to self-medicate the symptoms of depression or anxiety. Unfortunately, substance abuse causes side effects and in the long run worsens the very symptoms they initially numbed or relieved as these can increase underlying risk for mental disorders.

Mental disorders are caused by a complex interplay of genetics, the environment, and other outside factors. If you are at risk for a mental disorder, drug or alcohol abuse may push you over the edge. Alcohol and drug abuse can make symptoms of a mental health problem worse. Substance abuse may sharply increase symptoms of mental illness or trigger new symptoms. Alcohol and drug abuse also interact with medications such as antidepressants, anti-anxiety pills, and mood stabilizers, making them less effective. It is important to note that addiction is common in people with mental health problems.

To obtain a better understanding of the direct relationship between mental disorders and suicidal behaviour, studies accounting for the effects of co-occurring mental disorders are essential. Risk factors for suicide among the young include the presence of mental illness- especially depression, conduct disorder, alcohol and drug abuse; previous suicide attempts and the availability of firearms in the home. In South Africa $60 \%$ of people who commit suicide are depressed while the suicide rate for children aged 10-14 years old has more than doubled over the last fifteen years. In South Africa the average suicide is 17.2 per 100000 (8\% of all deaths) which relates only to deaths reported by academic hospitals. Every day, an estimated 21 South Africans commit suicide and, according to experts, stress could be a significant contributing factor. Around $26 \%$ of cases were classified as severe, $31 \%$ as moderate and $43 \%$ as mild. Seedat explains that a case was rated as serious when the person was dependent on alcohol or drugs, had attempted suicide in the past 12 months, or when their functioning at work, or in their family or social life was severely impaired.

Studies done by University of KwaZulu-Natal researcher Lourens Schlebusch estimate that 7582 South Africans of suicide every year and 20 times that number attempt but fail to take their own lives. While reliable statistics and research as to why the rate is so high are scarce, Marthé Viljoen from the federation says new data suggests South Africans have unusually high stress levels. The few studies that have investigated these issues suggest that mental disorders predict the onset of suicide ideation, but may have weaker effects in predicting suicide plans or attempts among people with suicide ideation. (Kessler RC, Borges G, Walters EE. 1999, Nock MK, Beautrais A, et al. 2008 and Borges G, Kessler RC. 2008) recent study conducted by international research company Bloomberg, ranked South Africa as the second "most stressed out" nation in the world, following Nigeria. El Salvador was ranked third. Another study, conducted last year by IPSOS Global and Reuters, showed that up to $53 \%$ of South Africa's workforce does not take their allotted annual leave.

The findings from this study reveal that the presence of multiple disorders is associated with an increase in subsequent suicide attempt. This finding is consistent with a previous literature revealing that adults who had attempted suicide reported more than one mental disorder. (Ndosi NK \& Lyamuya EL. 2004 and Slap GB, \& Centor RM. 1989). "High stress levels have been linked to mental illnesses such as depression and anxiety, and can also lead to substance abuse. In severe cases, these problems can lead to a person becoming suicidal," says Viljoen. South Africa already has high rates of substance abuse with, for example, alcohol alone being the third-highest contributor to death and disability among citizens, according to a 2014 study published in the South African Medical Journal. Psychiatric illnesses in South Africa are on the rise with rates of major mental illnesses such as schizophrenia seeming to be stable but cases of depression and anxiety certainly escalating.

The most recent nationwide prevalence study for mental health was conducted from 2002 to 2004 and it found that South Africans have a 30\% chance of suffering from a mental disorder in their lifetime, with depression being the most common. To begin with, $61 \%$ of South Africans who 
Texila International Journal of Clinical Research

Volume 3, Issue 2, Dec 2016

seriously considered killing themselves and $70 \%$ who actually made a suicide attempt were found to have a prior mental disorder. Bivariate analyses revealed that individuals suffering from depression resulting from depression resulting from the stressful situations. Stott says she is "not surprised" that the country has such a high suicide rate. "We can see from the data that South Africans work too hard and don't take enough time off." A recent survey conducted by mental health awareness organization the South African Depression and Anxiety Group (SADAG) showed that, while a quarter of the more than 1000 respondents have been diagnosed with depression, they were likely to remain at work while experiencing symptoms. Stott says the "mounting claims" for depressive and stress-related conditions show that many South Africans "can't cope". "A lot of people don't even recognize they have a problem as stress becomes so normalized."

She further says people suffering from stress-related depression and anxiety should seek professional help before "stress turns into despair". "People should see a counselor or a psychologist to discuss their problems. Antidepressants can also help if a professional thinks it is necessary," says Stott. For people with access to medical aid, she says most schemes pay for 21 days of in-hospital psychiatric treatment regardless of the condition. It can be difficult to diagnose a substance abuse problem and a co-occurring mental health disorder such as depression, anxiety, or bipolar disorder. It takes time to tease out what might be a mental disorder and what might be a drug or alcohol problem. Complicating the issue is denial. Denial is common in substance abuse. It's hard to admit how dependent you are on alcohol or drugs or how much they affect your life. Denial frequently occurs in mental disorders as well. The symptoms of depression or anxiety can be frightening, so you may ignore them and hope they go away. Or you may be ashamed or afraid of being viewed as weak if you admit the problem.

Whether the mental health condition or substance abuse problem came first, recovery depends on treating both disorders since untreated co-occurring disorders can lead to major problems at home, work and in the day to day life. Individuals with mental disorders and their families may delay seeking professional help because they do not realize their condition can be treated; may not afford treatment; or fear being labeled and stigmatized. So it's important for people to be encouraged to seek help. Recovery depends on treating both the addiction and the mental health problem. Recovering from co-occurring disorders takes time, commitment, and courage. It may take months or even years but people with substance abuse and mental health problems can and do get better. Combined treatment is best. Your best chance of recovery is through integrated treatment for both the substance abuse problem and the mental health problem. This means getting combined mental health and addiction treatment from the same health provider or team.

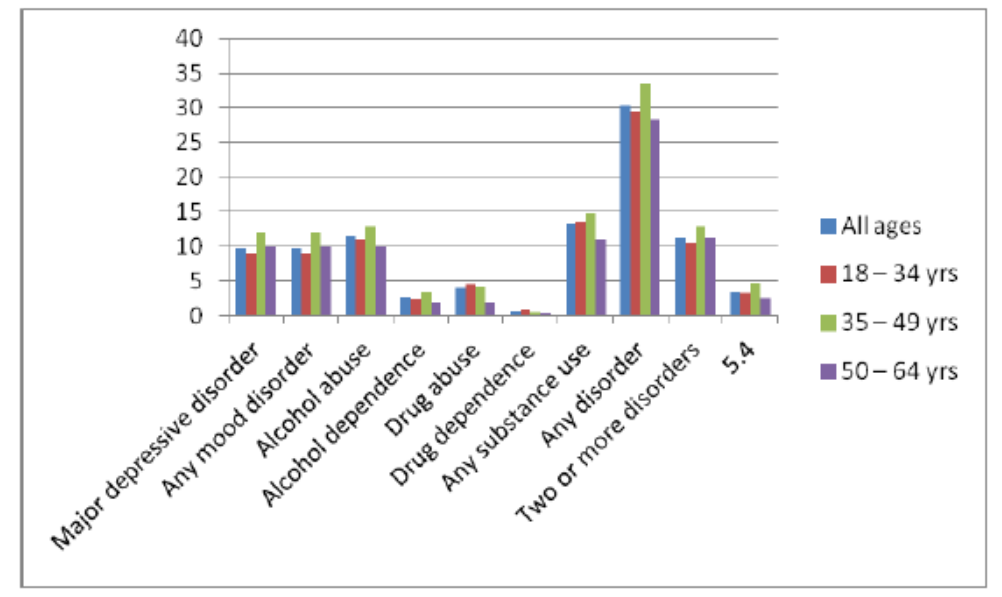

35-49 years had the highest prevalence in all the categories of disorders apart from drug abuse. Age groups 35-49 and 50-64 years had the highest prevalence of having two more disorders. Alcohol dependence and drug dependence had the lowest prevalence in all the age groups. As noted, some 
people might abuse substances to cope with mental health problems. This connection means that by providing effective and timely mental health treatment, we might be able to impact problems with substance abuse. Alternately, given that substance abuse can trigger mental health problems, addressing substance abuse may help reduce distress and the risk of the other concurrent disorders. When opportunities for prevention and early intervention are missed, people who have both mental health and substance use problems may need treatment that can address both the problems.

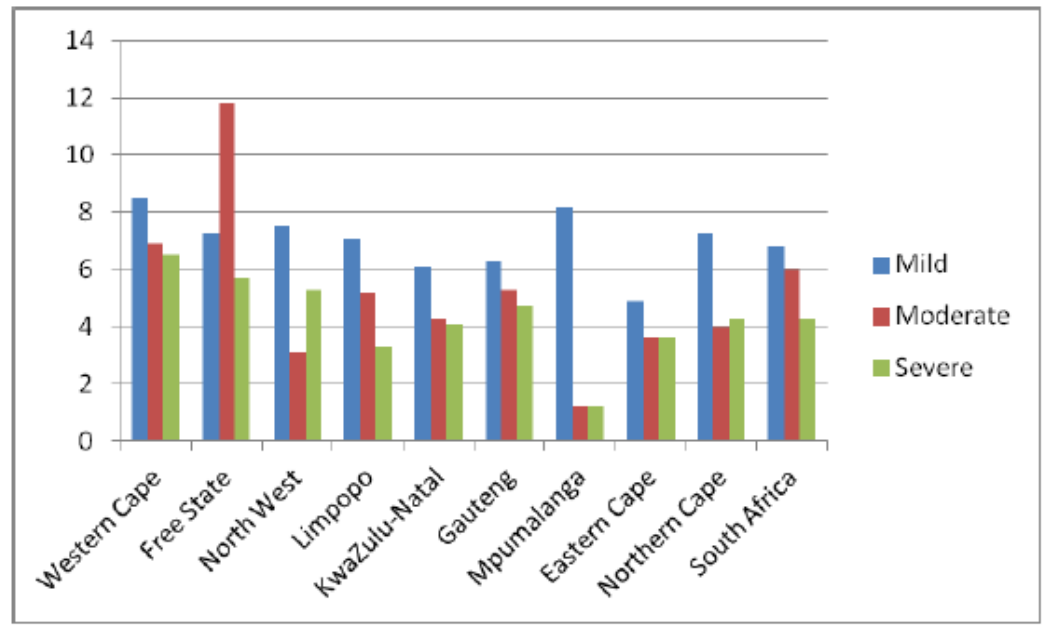

Fig 3

Significant differences in lifetime prevalence of mental disorders occurred across the nine provinces, with the Western Cape having higher prevalence rates as compared to the overall national prevalence rates, this province has the highest prevalence of severe cases of common mental disorders, followed by Free State with the Eastern Cape and Mpumalanga having the lowest prevalence. These provincial differences held for all mental disorders except impulse disorders, where the lowest provincial rates were found in Mpumalanga $(0.2 \%)$ and the Eastern Cape $(0.1 \%)$. Significant differences in prevalence with age occurred with panic disorder and generalised anxiety disorder (showing a general monotonic increase in rates with age) and drug abuse and drug dependence (showing a tendency to peak at 35 - 49 years). There was a marginally significant increase in the rate of mood disorders with increasing age. The situation is further evident based on the fact that $17 \%$ of children and adolescents in the Western Cape have a mental disorder and very few school-based mental health prevention programmes in place. (SASH 2009).

\section{The Socio-demographic trends of mental health situation}

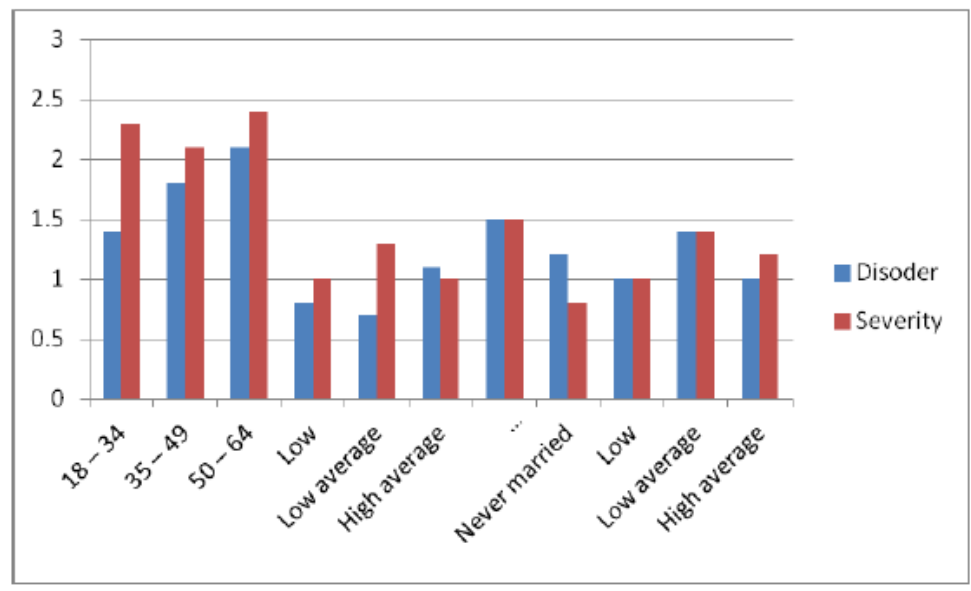

Fig 4 
Texila International Journal of Clinical Research

Volume 3, Issue 2, Dec 2016

Socio-demographic variables significantly related to onset of psychiatric disorders, women had a significantly higher risk than men of anxiety and mood disorders onset while men had a significantly higher risk of substance use disorders onset, and there were no significant associations with race. Furthermore, in an analysis that examined inter-cohort differences in demographic effects, no interactions with cohort were found for gender, race, and education, indicating that these effects have been stable over the generations included in the SASH survey. A significant positive relation between socioeconomic status (SES) indicators (e.g. income, education, wealth) and mental health was evident. Because SES and demographic variables are often confounded, disentangling their unique contribution to mental health and general psychological functioning is an important area of exploration.

Studies over the last 20 years indicate a close relationship between factors associated with poverty and mental health. The findings in fig 4 further support this notion and help to emphasize on the need for mental health efforts to target the low average earners are the most affected in terms of severity of the prevalence with high average earners having more disorder prevalence. Many studies indicate that individuals of lower SES report a greater number of stressors related to finances, relationships, transportation and employment than those of higher SES. (Gallo LC \& Matthews KA. 2003, McLeod JD \& Kessler RC. 1990, Matthews KA, Raikkonen K, Everson SA, et al. 2000 and Lantz PM, House JS, Mero RP, et al.2005). Residence in lower SES neighborhoods has also been linked to greater levels of stress. (Everson-Rose SA, Barnes LL, et al. 2011). The associations of psychiatric disorder with gender, the female gender was significantly associated with mood and anxiety disorders, male gender associated with substance use disorders which are consistent with those found in many other countries, whether low- or high-income.

In terms of the socio-demographic correlates the mental disorders the more the older population groups from 35 to 64 with plans for severe treatment targeting mostly the 35-49 age groups. It was further noted the mental disorders were highest among the age 50-64 group with 18-34 experiencing higher levels of severe disorders as compared to 35-49. Since most individuals with alcohol problems initiate drinking during adolescence, a period when not only the body is changing dramatically, but behavioural, cognitive, emotional, and attitudinal changes also take place (Dawson, 2000; Grant et al., 2006; Maggs and Schulenberg, 2005; Semlitz \& Gold, 1986). Repeatedly using alcohol (frequency or quantity) at such a critical stage in life may result in detrimental effects on brain development. It is important to lay more emphasis on the young people by targeting most of the preventive interventions towards this group. This is because youth with better mental health are physically healthier, demonstrate more socially positive behaviours and engage in less risky behaviour (Resnick, 2000). Conversely, youth with mental health problems, such as depression, are more likely to engage in health risk behaviours (Brooks et aI2002).

Other findings may, in terms of income severity was highest amongst the low average earners with high average earners experiencing the highest disorder prevalence and the low income earners experiencing lowest prevalence both in terms of disorders and severity. This points to the importance of local factors; the lack of an association between very low income and substance use disorders suggests the possibility that at least some disposable income is required for the purchase of alcohol which is the most commonly misused substance in South Africa and other substances.

In terms of levels of education the category with the low average education had the highest prevalence of both disorders and the severity of the disorder prevalence. This is consistent with several studies which have shown that socioeconomically disadvantaged individuals tend to experience more distress following a stressor than their more advantaged counterparts. (Collins JW Jr, David RJ, Symons R, et al. 1998, Chen E \& Matthews K. 2001 and Cohen S, Doyle WJ, Baum A.2006). The overall findings of the study indicated that although socioeconomically disadvantaged individuals reported fewer stressors, the stressors that they experienced were of greater severity. (Grzywacz JG, Neupert SD, et al., 2005). Therefore necessary efforts need to be made to target this group as opposed to the current held norm that the poor are the ones that suffer most in relation to 
mental disorders. This is consistent with levels of education where the people with low average were the most affected in terms of severity and disorder prevalence.

\section{Conclusion}

The study concludes that mental health and substance abuse problems are common and come at an enormous cost to individuals, families, communities and systems. The links between mental health and substance abuse issues are complex, they might develop independently as a result of common risk factors or one might lead to the other as a result of self-medication or prolonged distress. The most effective and efficient way to address co-occurring mental health and substance abuse disorders is to stop them before they start. Prevention of and early intervention for mental health and substance use problems is best, but when concurrent disorders develop, they require specialized intensive services. For South Africa to alleviate its overall healthcare burden, mental health more especially cases of substance and alcohol abuse need to be made a priority. Despite limitations, this paper provides further insight into the relationship between alcohol and substance abuse, suicidal behaviour and mental disorders in South Africa.

People with mental and psychosocial disabilities are a vulnerable group as a result of the way they are treated by society. They are subjected to stigma and discrimination on a daily basis, and they experience extremely high rates of physical and sexual victimization. Frequently, people with mental disabilities encounter restrictions in the exercise of their political and civil rights, and in their ability to participate in public affairs. They also are restricted in their ability to access essential health and social care, including emergency relief services. Most people with mental disabilities face disproportionate barriers in attending school and finding employment. As a result of all these factors, people with mental disability are much more likely to experience disability and die prematurely, compared with the general population. It is hoped that the findings will aid in the development of appropriate preventive interventions for the target population in South Africans that is more at risk for alcohol and substance abuse and suicide. For these programmes to be more successful the importance of co-morbidity and analogous programs are necessary in South Africa.

The best treatment for co-occurring disorders is an integrated approach, where both the substance abuse problem and the mental disorder are treated simultaneously. The dual diagnosis and treatment for co-occurring substance abuse and mental health disorders can be further improved on by setting up a substance abuse and treatment database of private and public substance abuse treatment facilities that can help people be able to easily be able to access services. Setting up of support groups for substance abuse and co-occurring disorders. These groups are very helpful, not only in maintaining sobriety, but also as a safe place to get support and discuss challenges. Also helping drug and substance addicts to become involved with supported employment and other services that may help them in the recovery process and also providing them with a counselor trained in integrated dual diagnosis treatment to provide special counseling specifically designed for people with dual diagnosis. This can be done individually, with a group of peers, with your family, or with a combination which will in turn help these addicts to identify and develop their own recovery goals.

According to the World Health Organization 2002 report about $90.0 \%$ of mental disorders are nonpsychotic disorders. Such disorders due to their high prevalence in the general population $(20.0 \%$ $30.0 \%$ ), are usually called common mental disorders (CMD). These are mainly characterized by the presence of symptoms of depression and anxiety, and various nonspecific and somatic complaints (Goldberg DP, Huxley PY. 1992). CMD affect individuals in different age groups and, when present, may be early and less specific manifestations of more serious mental disorders, also impairing the social relationships and school performance in the youth population. Mental disorders have emerged as major challenges health services must face, hence to be able to address the challenge it is important to identify and treat mental health or substance abuse problems early. Often, before the formal diagnosis of a psychiatric disorder, it is already possible to find evidence of psychic suffering during clinical practice. Therefore development of early identification of CMD and its main risk factors, 
Texila International Journal of Clinical Research

Volume 3, Issue 2, Dec 2016

adequate screening, prevention and intervention tools would benefit from a more in depth understanding of mental disorders as a risk factor for suicide can contribute to specific interventions and a better prognosis.

\section{Limitations of this study}

This study has a number of limitations, this being a secondary analysis of an existing data base, there were limited data available to be able to make conclusive findings. These sentiments are shared by Lund who states that there is an urgent need of conducting a nationally representative prevalence study of mental disorders across the age range, including children, adolescents, adults and older adults. The other limitation was on time and resources to be able to do a comprehensive literature review. In future there is need to conduct more regular national survey on mental health.

\section{References}

[1.] Borges G, Angst J, Nock MK, Ruscio AM, Kessler RC. Risk factors for the incidence and persistence of suicide-related outcomes: a 10-year follow-up study using the National Comorbidity Surveys. J Affect Disord 2008 ; 105 (1-3): 25-33.

[2.] Cohen S, Doyle WJ, Baum A. Socioeconomic status is associated with stress hormones. Psychosom Med 2006; 68: 414-20.

[3.] Collins JW Jr, David RJ, Symons R, et al. African-American mothers' perception of their residential environment, stressful life events, and very low birth weight. Epidemiology 1998; 9: 286-9.

[4.] Chen E, Matthews K. Cognitive appraisal biases: an approach to understanding the relation between socioeconomic status and cardiovascular reactivity in children. Ann Behav Med 2001; 23:101-11.

[5.] Dube, S.R., Miller, J.W., Brown, D.W., Giles, W.H., Felitti, V.J., Dong, M.,\& Anda, R.F. (2006). Adverse childhood experiences and the association with ever using alcohol and initiating alcohol use during adolescence. Journal of Adolescent Health, 38, 1-10.

[6.] Edwards, V. J., Holden, G. W., Felitti, V. J., \& Anda, R. F. (2003). Relationship between multiple forms of childhood maltreatment and adult mental health in community respondents: results from the adverse childhood experiences study. American Journal of Psychiatry, 160, 1453-1460.

[7.] Everson-Rose SA, Barnes LL, et al. Neighborhood socioeconomic conditions are associated with psychosocial functioning in older black and white adults. Health Place 2011; 17:793-800.

[8.] Gallo LC and Matthews KA. Understanding the association between socioeconomic status and physical health: do negative emotions play a role? Psychol Bull 2003; 129:10-51.

[9.] Goldberg DP, Huxley PY. Common mental disorders: a bio-social model. London: Tavistock/ Routledge; 1992.

[10.] Grzywacz JG, Almeida DM, Neupert SD, et al. Socioeconomic status and health: a micro-level analysis of exposure and vulnerability to daily stressors. J Health Soc Behav 2004; 45:1-16.

[11.] Knapp, M., McDaid, D., \& Parsonage, M. (2011). Mental Health Promotion and Mental Illness Prevention: The Economic Case. 15972. Department of Health, London, UK.

[12.] Inge, P., Arvin, B., Victoria, C., Sithembile, M., Crick, L., Sharon, K., \& ... the Mental Health and Poverty Research Programme, C. (2009). Planning for district mental health services in South Africa: a situational analysis of a rural district site. Health Policy \& Planning, 24(2), 140. Retrieved from EBSCOhost. p. 141.

[13.] Joanna Saisan, M.S.W., Melinda Smith, M.A., and Jeanne Segal, Ph.D. Last updated: October 2016.

[14.] Kessler RC, Borges G, Walters EE. Prevalence of and risk factors for lifetime suicide attempts in the National Comorbidity Survey. Arch Gen Psychiatry 1999 ; 56 (7):617-26.

[15.] Lantz PM, House JS, Mero RP, et al. Stress, life events, and socioeconomic disparities in health: results from the Americans' Changing Lives Study. J Health Soc Behav 2005; 46:274-88.

[16.] Matthews KA, Raikkonen K, Everson SA, et al. Do the daily experiences of healthy men and women vary according to occupational prestige and work strain? Psychosom Med 2000; 62:346-53. 
[17.] McCreary Centre Society. (2012). Mental Health and Substance Use: A BC Adolescent Health Survey 2008 Fact Sheet. McCreary Centre Society. McCreary Centre Society: Vancouver. http://www.mcs.bc.ca/pdf/AHS4_Mental_Health_Substance_Use_Factsheet.pdf.

[18.] McLeod JD, Kessler RC. Socioeconomic status differences in vulnerability to undesirable life events. J Health Soc Behav 1990; 31:162-72.

[19.] Miller, T. \& Hendrie, D. (2008). Substance abuse prevention dollars and cents: a cost-benefit analysis. Center for Substance Abuse Prevention, Substance Abuse and Mental Health Services Administration, DHHS Pub. No. (SMA) 07-4298. Rockville, MD.

[20.] Ndosi NK, Mbonde MP, Lyamuya EL. Profile of Suicide in Dar es Salaam. East African Medical Journal 2004;81: 207-11.

[21.] Nock MK, Borges G, Bromet EJ, Alonso J, Angermeyer M, Beautrais A, et al. Cross-national prevalence and risk factors for suicidal ideation, plans and attempts. Br J Psychiatry 2008 ; 192(2):98-105.

[22.] Slap GB, Vorters DF, Chaudhuri S, Centor RM. Risk factors for attempted suicide during adolescence. Pediatrics 1989; 84: 762-72.

[23.] World Health Organization. Towards a common language for functioning disability and health-ICF. Geneva: World Health Organization; 2002. 


\title{
Review on Multiple Ethical Review in North-South Collaborative Research: the Experience of the Ebola-Tx Trial in Guinea
}

\author{
Article by Labban Fredrick Sichivula \\ M.Sc Clinical Research, Texila American University, Zambia \\ Email: labbans25@yahoo.com
}

\section{Introduction}

For the purposes of this paper, the review of the article titled"Multiple ethical review in NorthSouth collaborative research: the experience of the Ebola-Tx trial in Guinea" published in 2016 in the Indian journal of medical ethics, will focus on the following subtitles; the literature review, article summary and structure. In critiquing this article, the author of this paper will explore the credentials and work of the article's author to establish its authority and accuracy. We will deal with the time surrounding the publication of the article as well as the current references cited in the article. The objectivity of the article will be considered in terms of biasness, consideration of holistic information surrounding the subject and how the author navigated through the various issues to remain objective. The article'sstability will be reviewed based on the type of publication. The author will also zero in on the tables to point out important and relevant information. We will also review the relevance of the content in relation to the title of the article. This paper will also point out some of the recent work being done related to multiple ethical reviews in research and at the end of this article review, a clear and concise conclusion will be given.

\section{Review of literature}

Double ethical review is defined as the review of a research proposal involving ethics committees in countries of research site and sponsor. The practice is aimed at minimizing double standard practice and enhance the protection of participants and the population (Ravinetto et al., 2010).

The difference in research standards, research interests and cultural differences precipitate the use of multiple ethical review to harmonise research ethics. Researchers have called for the need for ethical review collaboration between host and sponsor countries (Hyder et al., 2004). They point out that this reflects the desire for focused capacity development in ethical review. They also note the reluctance of somehost (African) IRBs to raise issues to deal with confidentiality, local language consent forms and letter for approval which sponsor countries significantly raised. There are three areas of concern in research done in developing countries that have been identified which may demand double ethical review; these include the standard of care that should be used in clinical trials, reasonable availability of interventions that are proven to be useful during research trials and the quality of informed consents (Emanuel, Wendler, Killen and Grandy 2004). The issues raised by the researchers necessitate cooperating partners to increase capacity building in the protection of research participants. However, some authorities have expressed concern over the use of multiple ethical review that this may cause delay in execution of the study. They reiterate that the process is over burdening and involve a lot of time resources and it duplicates the process (Improved Research Ethics Review Process for Studies done Across Nations 2012).

Most scholars involved in multinational research have advocated for coordinated dialogue among ethical committees in host and sponsor countries so as to harmonise the research ethics. These efforts would provide a platform to resolve conflicting issues and enhance capacity building which will in turn better protect research participants. 
Texila International Journal of Clinical Research

Volume 3, Issue 2, Dec 2016

\section{Article summary}

The purpose of this article aims to review the experiences of multiple ethical reviews in the Ebola treatment trial that was conducted in Guinea during the unprecedented outbreak of the infectious disease in 2014.

The authors outlined the various ethical committees/Institutional review boards in Guinea and European countries which were sponsors involved in the review process. The objective of the research was to assess double ethical review and evaluate the implementation of the World Health Organization background document of September 2014 ("flexible approaches are required to harmonise various review processes, and ensure that the various ethics committees can review the projects simultaneously and share and discuss the review outcomes with each other"). The article points out different recommendations that were made by multiple ethical committees and how they were incorporated in the final research protocol. The author points out that multiple ethical reviews brings out complimentary views that benefit the research participants and enhance acceptability of the research by the community. The researcher however, also highlightsthe conflicting views that were raised during ethical reviews and the challenges to reconcile them in the case of emergency outbreaks where time is a limiting factor. It was recommended that there should be dialogue among various ethics committees to harmonize the review process.

\section{Article structure}

The structure of the article was developed in accordance with the outlined format of the Indian Journal of Medical Ethics. It begins with a title limited to 15 words. The abstract is well presented beginning with a definition of "double ethical review" and objective of the study. It goes on to describe the Ebola-Tx trial protocol approval process on which basis this article stands. The methodology used is not clearly indicated. The author went on to outline the findings of the study, the conclusion and recommendations.

The article continues with a provision of background information regarding the use of double ethical reviews in externally sponsored research. Information on double ethical review in the EbolaTx trial is well described. On the first page, the authors' information box is given. Information on Ebola treatment options and protocol submissions to various ethical groups is given. The objective of the study are clearly outlined. The tables provided in the articles lists members of the Ebola-Tx consortium, ethical committees that reviewed the protocol. The methodology of the study is given in the main body and has provided specifics on what the researchers focused on.

The section for findings contains information on submission requirements that were requested by different ethical committees. It also provides information on the timeliness of the reviews which was crucial in the Ebola-Tx trial as well as a table to illustrate the time lapses from when the submissions were made to how long the ECs took to respond and when amendments where incorporated in the protocol. The contents of the review is included in form of a table and comments.

The discussion section is well formatted and a conclusion section is given. The article ends with acknowledgements, comment on funding and a statement of conflict of interest, notes and references.

\section{Authority}

The qualifications of the author is not given in the article. However, the credibility can be authenticated for the fact that the researcher works with a credible institution in clinical sciences department at the Institute of Tropical Medicine, Antwerp, Belgium. The author is a professional and is subscribed with the Indian journal of Medical Ethics that published the article on $25^{\text {th }}$ January, 2016. The co-authors are indicated to belong to the same institution as well as the other that is involved with Centre National de Formation et Recherche en Santé Rurale de Maferinyah, Conakry, Guinea. The authors were directly involved in the research process of Ebola Treatment trial and the have sound knowledge of the experience alluded to in the article. 


\section{Accuracy}

The information provided in the article is generated from a recent outbreak of Ebola in West Africa that captured the international attention in 2014. This attracted a lot of highly acclaimed world researchers to combat the Ebola virus disease outbreak and their experience is pointed out in the article under review. The article uses recent references and has made reference to several regulatory guidelines both international and local (Guinea). The information in the article can be verified using several media and academic sources in relation to the event that was widely publicized. The documents from World Health Organization is widely used to provide a credible background information on the use of convalescent plasma in Ebola therapy.

\section{Currency}

This is a recent article, published in January 2016. The events surrounding the basis of the research is within a period of two years from the time the Ebola treatment trial and time of publication. The references used range from 2001 to 2015. The post evaluation of the Ebola outbreak and treatment trial is still ongoing which supports that the article is current. The research protocol was first submitted to the ethics committees/institutional review boards on $22^{\text {nd }}$ November, 2014. The final draft of the protocol referred to in the article was approved by several ethical committees between 12 and 26 January, 2015 leading to the first online publication of the article in 2016.

\section{Relevance of the content}

This article was aimed at assessing the concept of multiple ethical reviews in order to make recommendations for research conducted during public health emergencies. It is applicable in the process where research is conducted is one country whereas the sponsors are from another country. The article generates important issues researchers are likely to face in a similar setting. These issues include, acceptability of the research by participants and the community, capacity building for local researchers and most of all, better protection of participants. It is also applicable in resolving conflict of interest between sponsors from another country and the local community where the research is conducted. With enhanced communication among different ethical committees, it would share knowledge and harmonise ethical review processes.

\section{Objectivity}

The author of the article discussed the pros and cons of multiple ethical reviews without bias. The article contains a statement declaring conflict of interest so as to remain as objective as possible. The research is based on lived experiences and supporting guidelines from research regulators. Referencing from other academic literature of different views on multiple ethical review has been exercised to eliminate bias. The conclusion of the researchers in the article points out the conflicting issues that were raised by different ethical review committees and indicates how each issue was resolved to ensure compliance. The recommendations given in the conclusion is in line with harmonization of ethical reviews as outlined by World Health Organization.

\section{Stability}

The article was published online by an establishedinternational academic journal "The Indian Journal of Medical Ethics" Vol I No 2 between April-June of 2016 whose database is stable. The journal has specific guidelines on the criteria of articles they accept and publish. It requires subscription of membership for contributors.

\section{Analysis of table}

The first table given in the article lists the various members of the Ebola treatment consortium. These institutions range from universities, research organizations, regulatory organization and World Health Organization which is the major world body dealing with Health issues. 
Texila International Journal of Clinical Research

Volume 3, Issue 2, Dec 2016

The second table is a list of multiple ethical committees that were involved in the protocol review process. These committees are station in both European and African countries as well as WHO.

The third table indicates different time intervals that the ethics committees took to review the protocol upon submission and for the re-submission of amendments.

The last table is a summary of multiple issues that were raised by the ethical review committees and how the comments were treated to comply with the recommendations.

\section{Recent advances related to the topic}

Scholars in the field of clinical research are working on means that will promote speedy implementation of research especially in public health emergencies like the "Ebola outbreak." Many lessons have been leant over the years on how study delays can be minimized. Schopper et al (2015) have suggested five innovative practices in research; introduction of policy to exempt a posteriori analysis of routinely collected data, preapproval of emergency protocols, general ethical approval of routine surveys, new framework to guide ethics review and evaluating the impact of approved studies. They point out that these measures would enhance the process of research. With the increasing demand for global health training, Crump and Surgarman (2010) point out that there must be guideline in place to foster best ethical practices in training of students who wish to gain experience in different countries. With the current advancement in the medical field of organ transplant Erin and Arris (2003) proposed the ethical support of the organ sale markets. They have suggested the use of a single marked of human organs within a confined market place. In the world of sport, Kayser, Mauron and Miah (2007) suggest that rather than striving for eradication of doping in sports, which appears to be an unattainable goal, a more pragmatic approach aimed at controlled use and harm reduction may be a viable alternative to cope with doping and doping-like behavior. There is a marked underrepresentation of countries in high-impact general medical journals. This has high ethical implications concludes Sumathipala, Siribaddana and Patel (2004). This conclusion resonates with the idea of enhancing capacity building research ethics for researchers in the developing countries. Beeson and Lippman (2006) have expressed concern over the harvesting of multiple eggs [that] often involve the administration of drugs that have not been approved for this purpose. These issues highlighted are meant foster improved research ethics in stem cell research.

\section{Conclusion}

The review of the article has been summarized and critically analyzed. The abstract provides the significance of the study, objective, findings, conclusion and recommendations.

The background outlines the use of double ethical review in multinational studies in situations. In this review, the research was conducted in Guinea while sponsors were from Belgium and other countries. The double ethical review is meant to increase better protection for participants and to harmonize research ethics.

The Ebola-Tx trial involved the interventional use of convalescent plasma in the treatment of Ebola Virus Disease (EVD). The study protocol was submitted to various ethics committees who reviewed and made recommendations that became part of the final protocol. The article was focused on assessing the experience of double ethical review and to make recommendations on this practice in future public health emergencies.

The critical review of this article recognizes the meticulous approach the author used to discuss the merits and demerits of double ethical review. The findings show that multiple ethical review may increase the complexity of the protocol especially when there are conflicting issues raised by the IRBs. The author also points out that the practice increases better protection of participants when ethical committees involved have open dialogue in the review process and promotes capacity building. The findings in the article provides an in-depth insight in the approval of research protocols involving multinational organizations. The host country and sponsors country's research organizations need coordinated efforts to harmonise research ethics as recommended by WHO. 


\section{References}

[1] Beeson, D., \& Lippman, A. (2006). Egg harvesting for stem cell research: medical risks and ethical problems. Reproductive BioMedicine Online, 13(4), 573-579.

[2] Crump, J., Sugarman, J. \& Working Group on Ethics Training (WEIGHT.) (2010). Ethics and best practices guidelines in Global Health. The American Journal of Tropical Medicine and Hygen, 83(6), 1178-1182

[3] Hyde, A.A, Wali, S.A., Khan, A.N., Tech, N. B., Kass, N.E., \& Dawson, L. (2004). Ethical review of Health research; a perspective for developing country researchers. Journal of Medical Ethics, 30(1), 68-72.

[4] htt://globalhealthtrials.tghn.org seen on 14.11.2016.

[5] Erin, C. A., \& Harris, J. (2003). An ethical market in human organs. Journal of medical ethics, 29(3), 137138.

[6] Emanuel, E.J., Wendler, D., Killen, J., \& Gandy, C. (2004). What makes clinical research in developing countries ethical? The benchmark of ethical research. Journal of Infectious Diseases, 189(5), 930-937.

[7] Kayser, B., Mauron, A., \& Miah, A. (2007). Current anti-doping policy: a critical appraisal. BMC medical ethics, 8(1), 1.

[8] Ravinia, R., Tinto, H., Rouamba, A., Talisuna, Adoke, Y., Kadima Ebeja, A., Maketa, V., Grieftens, K.P., Buve, A. \& Crawley, F. (2010). Health research: The challenges related to ethical review and informed consent in developing countries. Giornale Italiano Di Medicina Tropicale, 15.N.1-4, p15.

[9] Sumathipala, A., Siribaddana, S., \& Patel, V. (2004). Under-representation of developing countries in the research literature: ethical issues arising from a survey of five leading medical journals. BMC Medical Ethics, $5(1), 1$.

[10] Schopper, D., Dawson, A., Upsani, R., Ahmad, A., Jesani, A., Ravinetto, R., Segelid, M.J., Sheel, S., \& Sigh, J., (2015). Innovation in research ethics governance in humanitarian settings BMC medical ethics. DOI 1186/5 $12910-015-0002-3$

\section{Source}

De Crop, M., Delamou, A., van Griensven, J., \& Ravinetto, R. (2016). Multiple ethical review in North-South collaborative research: the experience of the Ebola-Tx trial in Guinea. Indian journal of medical ethics, 1(2). Viewed on $28^{\text {th }}$ October 2016.

http://www.issuesinmedicalethics.org/index.php/ijme/article/view/2342 


\title{
Effect of Structured In-service Education on Knowledge level among Nurses Working in Surgical Units in Private Tertiary Hospital Islamabad, Pakistan
}

\author{
Article by Nuzhat Sultana \\ Ph.D Nursing, Texila American University, Pakistan \\ Director Nursing Education Services, Shifa International Hospital Islamabad, Pakistan \\ Email: nuzhatsultana@hotmail.com
}

\begin{abstract}
Background: Colostomy care is an important nursing intervention to ensure patient and family centered care. A number of patients go through colostomy procedure annually. The assessment of nurse's knowledge of colostomy care has not been conducted before in study hospital. Therefore, it is important to assess the knowledge of nurses caring for colostomy patients.

Purpose: The purpose of this study was to determine the effect of structured in-service education on knowledge level among nurses working in male and female surgical units.

Method: Participatory Action Research (PAR) approach was selected using pre and post - test method to assess the nurse's knowledge regarding colostomy care after the approval of IRB in a tertiary hospital, Islamabad, Pakistan.

Sample: The purposive sampling technique was used to select the study participants $(n=39)$.

Finding: The study findings showed that in pre-test nearly all of the study participants ( $n=36$, 92.3\%) received the score between 0-10 (poor and average). While in post-test the majority of the study participants ( $n=34,87.18 \%)$ received the score between 21-25 (excellent). Pair t-test showed the mean score in pre-test $6.08 \pm 3.74$ and post-test mean score 22.38 \pm 5.54 , P-value $<0.05$, Paired ' $t$ ' test revealed that the nurses gained significant knowledge after the structured in-service education.

Conclusion: The study concluded that there is an intense need for nurse managers to pay attention to this special area of nursing care by developing the mechanism for nurse's in-service education on regular basis for colostomy care. Moreover, future researches are warranted to validate the findings of this study.
\end{abstract}

Keywords: Colostomy care, pre-test, post-test, nurse's knowledge, assessment, in-service education.

\section{Introduction}

Nurses play crucial role in caring short term and long-term colostomy patients and preparing them for self- care and coping with changed health status. Appropriate nurse's knowledge of managing colostomy patient is essential. Nurses are involved in holistic care of colostomy patients, preoperative care, post operative care, care of colostomy, assessing the peristalsis movements and stool characteristics, monitoring and managing complications, colostomy irrigation, optimal nutrition, patient and family education (Bhzeh, Teleb, Mahmoud, \& Soliman, 2013). According to the literature, colostomy is a surgical procedure that takes out one end of large intestine and colon is side tracked through an incision in the abdominal wall to form a stoma. A stoma is the opening in the skin where a small bag for collecting feces attached. People with temporary or long-term colostomies have pouches attached to their sides where feaces collect and can be easily disposed off (Bhzeh, et al., 2013; Burch, 2011; Krans, January 8, 2016). Colostomy is named according to the site of opening: it may be ascending, descending, transverse or sigmoid colostomy cited in (Bhzeh, et al., 2013).

A number of patients undergo colostomy procedure every year to treat and reduce patient's pain and discomfort. On the other hand, this procedure causes stress and agony for patients, as a result of skin irritation $76 \%$, pouch leakage $62 \%$, unpleasant odor $59 \%$, discontinue of enjoyable activities 
Texila International Journal of Clinical Research

Volume 3, Issue 2, Dec 2016

54\%, and depression/anxiety 53\%, cited in (Dabirian, Yaghmaei, Rassouli, \& Tafreshi, 2011). It is estimated that about 13,000 colostomy procedures are performed annually in Canada that needs patient centered care in the hospitals (Recalla et al., 2013). Literature supports that nurses taking care of colostomy patients must be well equipped with required knowledge related to colostomy care to provide high standard care based on patient's need (Bales, 2010; Bhzeh, et al., 2013; Lebona, Jasmine, Lakshmi, \& Indira, 2016).

A study conducted in Iran reported that living with stoma affects the overall quality of life. Therefore, health care professional must be knowledgeable to cater the patients physical and psychological educational needs (Anaraki et al., 2012). Previous literature also indicated that nurses can play significant role in physical, psychological, economical, social and many other aspects of care in colostomy patients provided they are adequately updated through in-service education related to colostomy care (Sinha, Goyal, Singh, \& Rana, 2009).

A recent literature supports the importance of addressing nurses in-service educational needs related to colostomy care. A qualitative study conducted on 32 gynecologic cancer patients with colostomy in Turkey indicated that patients felt the high need for psychological support from nurses to minimize the fear, worry and anxiety and women going under colostomy were concerned about their body image, self-esteem, social activity and sexuality while in need for support from nurses (Gül, Ayhan, \& Tevfik, 2013). Literature further states that colostomy patients go-through lot of stress and feeling undignified. The stoma is usually red, inflamed, and outsized immediately after the surgery and horrible for the patient to look at for the first time. The emotional distress, along with physical problems, pain, isolation from others, and fear of death, will inevitably reduce quality of life further that may be looked after by the nurses who are better prepared with current knowledge related to colostomy care (Dabirian, et al., 2011).

Moreover, nurses play important role in patients teaching about changing colostomy bag, fear of leakage, disruption in sleep (Baldwin et al., 2009), also help patients feeling stigmatize (Smith, Loewenstein, Rozin, Sherriff, \& Ubel, 2007), helping young patients feelings embarrassed and frustrated due to their ostomy (Recalla, et al., 2013). Suicidal ideation after colostomy procedure was reported as for men 5.5\% and female $8.1 \%$ who needed nurses support (Krouse et al., 2009). Colostomy patients suffering with depression and facing multiple problems seek help from nurses (Thamilselvam, Khairuzi, \& Fadzli, 2013). A recent study conducted in Pakistan reported the lack of trained nurses in colostomy care (Zakri, Shah, Khaliq, \& Asghar, 2010). Managing colostomy patient is a complex situation, it is important that time to time nurses knowledge must be up-dated to help the patient to manage their colostomy care (Ardigo \& Amante, 2013).

A recent descriptive study conducted in Assiut University Hospital, Egypt to evaluate the knowledge and practice of 60 nurses in selected area related to colostomy care established that $82.7 \%$ nurses had satisfactory level about the definition of colostomy, 9.6\% knew the types of colostomy, $26.9 \%$ had satisfactory level of causes of colostomy, $7.7 \%$ understood the physiology of colon, $46.2 \%$ recognized anatomy of colon and $11.5 \%$ had satisfactory level of knowledge about most favorable nutrition for colostomy patients (Bhzeh, et al., 2013). Another study conducted on 189 nurses to assess nurses knowledge on pre-test and post-test reported the significant effect and enhanced knowledge and confidence on the quality and provision of care for ostomy wound management (Bales, 2010). Here colostomy patients need better-prepared nurses who can help them to cope with changed health condition.

Nursing care of colostomy is significant action to ensure quality of care (Bhzeh, et al., 2013). A recent literature review conducted by Registered Nurses' Association of Ontario supports that colostomy patients stated that the early interventions from nurses to their stoma was an marker of how they would live with their stoma (Recalla, et al., 2013). Another study suggested that nurses working with colostomy care must attained colostomy courses, seminars and workshop to enhance their knowledge and skills related to colostomy care (Thamilselvam, et al., 2013). 
Many other researches also identified the need to assess the nurse's knowledge regarding colostomy care (Ardigo \& Amante, 2013; Bhzeh, et al., 2013; Lebona, et al., 2016). A recent study conducted to assess the nurses knowledge $(n=30)$ regarding colostomy care reported that $(n=8,53 \%)$ had inadequate knowledge, $(n=6,40 \%)$ had moderately adequate knowledge and $(n=1,6.7 \%)$ had adequate knowledge. The study concluded that majority of staff nurses had inadequate knowledge regarding colostomy care (Lebona, et al., 2016).

In the study hospital, 5-6 average colostomy procedures are done every month that indicates the need to assess the nurse's existing knowledge related to colostomy care and determine the effect of structured in-service session. On literature search via scholarly article, PubMed, Wiley online library and Research Gate researcher found very few studies on the selected topic indicating the need to conduct the study on selected topic. Moreover, there is no such study conducted before at the study hospital. Therefore, current study was planned to assess the nurse's knowledge and determine the effect of structured in-service education regarding colostomy care before and after the training session.

\section{Objective of the study}

The objective of the study was to assess the knowledge of nurses related to colostomy care before and after the structured in-service education session.

\section{Research question}

To fulfill the purpose of the study following research question was formulated:

What is the difference in nurse's knowledge level before and after the structured in-service education session?

\section{Inclusion/ exclusion criteria}

All nurses working in male and female surgical units and willing to participate in the study were included in the study. Nurses on annual/sick leave and not willing to participate in the study were excluded from the study.

\section{Hypothesis}

$\mathrm{H}_{0}$ : There will be no significance difference between the pre-test and post-test knowledge score related to colostomy care among the study participants.

$\mathrm{H}_{1}$ : There will be significance difference between the pre-test and post-test knowledge score related to colostomy care among the study participants.

\section{Methodology}

\section{Study design}

The selection of research design deals with critical decision making that must be made to conduct the research study. Research approach is a systematic and objective method of discovery with empirical evidence and rigorous control. Participatory Action Research (PAR) approach was selected to conduct the study. As the nurses, training need was identified by the nurse manger surgical units and communicated to the researcher who is responsible to facilitate the nurse's trainings and their professional development across the hospital based on identified educational need of the staff through their immediate supervisor/ manager. According to Polit and Beck PAR is teamwork between researcher and study participants in identification of the problem and finding its solution. The aim of PAR is realization and action including knowledge enhancement as researcher put efforts to empower the study participants in developing and using innovative information (Polit \& Beck, 2004). 
Texila International Journal of Clinical Research

Volume 3, Issue 2, Dec 2016

\section{Independent variable}

The independent variable in this study is structured in-service educational workshop/session for nurses related to colostomy care.

\section{Dependent variable}

The dependent variable in this study is nurse's knowledge related to colostomy care.

Target population

The population of this study was all nurses working at the study hospital.

\section{Setting}

This study was conducted in Shifa International Hospital, Islamabad, Pakistan that is 500-bedded private tertiary health care facility comprised of more than 100 critical beds (medical and surgical including peads and NICU).

\section{Sampling and sample size}

Purposive sampling technique was adopted to select the study participants. All nurses $(n=50)$ working in male and female surgical units taking care of colostomy patients were invited to participate in the study. However, the actual response rate was $78 \%(n=39)$ who were available to participate in the study.

\section{Data collection tool}

Data was collected using self-developed tool based on current literature review in the form of pretest and post-test to assess/reassess the nurse's knowledge related to colostomy care. Tool consisted of two parts: Part 1: Socio-demographics characteristics of nurses; Including age, gender, academic qualification and work experience. Part 2: Included knowledge related questions; five subjective questions formulated to assess the nurse's knowledge related to colostomy care. Question no.1: posed to assess the knowledge related to definition of colostomy. Question no. 2: contained to write the types of colostomy. Question no. 3: comprised to list at least five teaching needs of colostomy patient. Question no. 4: related to food that should be recommended to colostomy patient that makes the stool soft. Question no 5: Name Supplies used for Colostomy Care and listing 3 competencies required for Colostomy Care. Each question allocated five marks as of evaluation criteria. Each item was given the score between $0-5$ according to the response of the study participants. Item wise rating indicated the various level of knowledge score from 0-5 (poor), 6-10 (average), 11-15 (good), 16-20 (very good) and 21-25 (excellent).

Two expert nurses in the field did content validity and face validity of the tool. By the end of this phase, a pilot study was carried out on $(n=4 ; 10 \%)$ of the sample to test the feasibility of the study and applicability of the tool. There was no modification made in the pre-test after the pilot study. Therefore, the study participants of the pilot study were included in the actual study as well.

\section{Ethical consideration}

The ethical aspects were considered by seeking formal approval from IRB of the study hospital before the study begins. The nurses willing to participate were included in the study along with informed written consent. The participants were assured for the confidentiality and safety of the data. Moreover, the participants were having freedom to withdraw from the study any time during the study.

\section{Data collection process}

The data was collected by researcher in last week of June 2016. Four hours structured in-service session/workshop during the morning shift (10 a.m.-2 p.m.) was conducted using the pre-test and post-test approach. After the pre-test, a planned interactive training session was delivered to impart 
the knowledge related to colostomy care among the study participants. Content was covered in detail by the responsible Nurse Manager and clinical instructor facilitate by researcher. Power point presentation session was conducted for 45 minutes including pictorials, scenarios and related videos. Power point presentation: Hand-out and recommended sites for reading were also provided to each participant during the workshop as a resource material and were given enough time to access recommended sites on internet. Study participants were also assisted to watch videos related to colostomy care followed by question answers session for 30 minutes. At the end of the workshop, post- test was conducted comprising the same questions used in pre-test. Each response was given the score according to predetermined evaluation criteria by the field experts. Each item was scored between $0-5$ according to the response of the study participants.

\section{Data analysis}

Data was entered and analyzed by using SPSS version 16. Descriptive and inferential statistics was calculated according the type of variables. Frequencies and percentages for nominal and categorical demographic variables were calculated. Mean and standard deviation was computed for participants' score in each item of knowledge related questions. Hypothesis was tested by using paired t-test. Paired t-test was applied on the mean of pre \& post-test score. Significance level was set at 0.05.

\section{Results}

\section{Demographic characteristics}

The sample constituted of nurses $(n=39)$ working in male and female surgical units. The socio demographic data of the study subjects were analyzed using descriptive statistics and were presented in terms of frequency and percentage. Majority of the participants $(n=26,66.7 \%)$ were females, aged between 21-30 Years $(n=25,64.1 \%)$. Education wise most of the participants $(n=35)$ had General Nursing 3 Years Diploma with additional one year specialty diploma in Pediatric/Psychiatric /Cardiology/ Midwifery and post RN BscN, while ( $\mathrm{n}=23,59 \%)$ had 1-10 years experience (Table 1).

Table 1. Socio demographic Variables $(n=39)$

\begin{tabular}{|l|l|l|}
\hline Variables & F & \% \\
\hline Gender & & \\
Female & 26 & 66.7 \\
Male & 13 & 33.3 \\
\hline Age & & \\
$21-30$ & 25 & 64.1 \\
$31-40$ & 8 & 20.5 \\
$41-50$ & 4 & 10.3 \\
$51-60$ & 2 & 5.1 \\
\hline Education & & \\
General Nursing 3 Years Diploma and one & 30 & 76.9 \\
year diploma ${ }^{1}$ & & \\
General Nursing plus Post RN BScN & 5 & 12.8 \\
BScN 4 Year & 4 & 10.3 \\
\hline Experience in Year & & \\
Fresh & 10 & 25.6 \\
1-10 & 23 & 59 \\
11-20 & 4 & 10.3 \\
$21 \&$ above & 2 & 5.1 \\
\hline
\end{tabular}

\footnotetext{
${ }^{1} 12$ Nurses had additional one year specialty diploma in Pediatric, Psychiatric, Cardiology \& Midwifery
} 
Texila International Journal of Clinical Research

Volume 3, Issue 2, Dec 2016

The mean score of question no. 1 in pre-test is $2.28 \pm 1.40$ and in post-test mean score 4.69 \pm 1.13 . Pvalue $<0.05$, Paired ' $t$ ' test showed that the nurses gained significant knowledge after the in-service education session. Question no. 2 the mean score of pre-test is $0.46 \pm 0.94$ and in post-test mean score 4.59 \pm 1.35 . P-value $<0.05$, Paired 't' test found the significant enhancement of knowledge in post-test. Question no. 3 the mean score in pre-test is $1.36 \pm 1.22$ and post-test mean score $4.15 \pm 1.51$, P-value < 0.05 , Paired ' $t$ ' test proved the significant increase in nurses knowledge after receiving the in-service education session. Question no. 4 the mean score of pre-test is $0.23 \pm 0.49$ and post-test mean score 4.44 \pm 1.37 . P-value $<0.05$, Paired ' $t$ ' test observed that the nurses gained significant knowledge after the intervention of in-service education session. Question no. 5 the mean score of pre-test is $1.74 \pm 1.76$ and post-test mean score $4.51 \pm 1.17$. P-value $<0.05$, Paired't' test established the significant improvement in nurses knowledge after attending the in-service session (Table 2).

Table 2. Pre-test \& Post-test Item Wise Scores

\begin{tabular}{|l|l|l|l|l|l|}
\hline \multirow{2}{*}{ Item questions } & \multicolumn{2}{|l|}{ Pre-test score } & \multicolumn{2}{l|}{ Post-test score } & $\begin{array}{l}\text { P-value } \\
\text { (Sig. 2- } \\
\text { tailed) }\end{array}$ \\
\cline { 2 - 6 } & Mean & $\begin{array}{l}\text { Std. } \\
\text { Deviation }\end{array}$ & Mean & $\begin{array}{l}\text { Std. } \\
\text { Deviation }\end{array}$ \\
\hline What is Colostomy & 2.28 & 1.40 & 4.96 & 1.13 & 0.000 \\
\hline Write Types of Colostomy & 0.46 & 0.94 & 4.59 & 1.35 & 0.000 \\
\hline $\begin{array}{l}\text { Identify at least 5 teaching } \\
\text { needs of Colostomy Patient }\end{array}$ & 1.36 & 1.22 & 4.15 & 1.51 & 0.000 \\
\hline $\begin{array}{l}\text { Name the food that soften } \\
\text { the stool }\end{array}$ & 0.23 & 0.49 & 4.44 & 1.37 & 0.000 \\
\hline $\begin{array}{l}\text { List supplies and 3 } \\
\text { competencies required for } \\
\text { Colostomy Care }\end{array}$ & 1.74 & 1.76 & 4.51 & 1.17 & 0.000 \\
\hline
\end{tabular}

Paired t-test was used to compare mean score

In overall pre-test nearly all of the study participants $(n=36,92.3 \%)$ received the score between 0 10 (poor and average); and only $(n=3,7.7 \%)$ of the participants received the score between 11-15 (good). While in post-test the majority of the study participants $(n=34,87.18 \%)$ received the score between 21-25 (excellent); while only few of the study participants $(n=3,7.7 \%)$ received the score between 16-20 (very good) and two participants who did not attempt the post-test scored zero (poor) (Table 3).

Table 3. Distribution of overall knowledge scores

\begin{tabular}{|l|l|l|}
\hline Knowledge (Score) & $\begin{array}{l}\text { Pre-test Frequency } \\
(\%)\end{array}$ & $\begin{array}{l}\text { Post-test Frequency } \\
(\%)\end{array}$ \\
\hline Poor (0-5) & $18(46.15)$ & $2(5.13)$ \\
\hline Average (6-10) & $18(46.15)$ & $0(0)$ \\
\hline Good (11-15) & $3(7.7)$ & $0(0)$ \\
\hline Very Good (16-20) & $0(0)$ & $3(7.69)$ \\
\hline Excellent (21-25) & $0(0)$ & $34(87.18)$ \\
\hline
\end{tabular}

The pair t-test knowledge score achieved by the respondents in results shows that the mean value of knowledge in pre-test is $6.08 \pm 3.74$ and at post-test $22.38 \pm 5.54$. P-value $<0.05$, Paired' $t$ ' test revealed that the nurses gained significant knowledge after the in-service education (Table 4). 
Table 4. Pair $t$ test of overall knowledge scores

\begin{tabular}{|l|l|l|l|}
\hline Pair & Mean & $\begin{array}{l}\text { Std. } \\
\text { Deviatio } \\
\mathrm{n}\end{array}$ & $\begin{array}{l}\text { P-Value (Sig. } \\
\text { 2-tailed) }\end{array}$ \\
\hline Pre-test & 6.08 & 3.74 & \\
\hline Post-test & 22.38 & 5.54 & 0.000 \\
\hline
\end{tabular}

\section{Discussion}

The study participants working in male and female surgical units $(n=39)$ were evaluated for nursing knowledge related to colostomy care. The socio demographic data of the study subjects were analyzed using descriptive statistics and were presented in terms of frequency and percentage. Majority of the participants (66.7\%) were females and 33.3\% male. Aged between 21-30 years 64.1\%; 31-40 years 20.5\%; $41-50$ years $10.3 \%$ and $51-60$ years $5.1 \%$. Education vise the most of the study participants (89.7\%) had General Nursing 3 Years Diploma with additional one year specialty diploma in Pediatric/Psychiatric/Cardiology/ Midwifery and two years post RN BscN, while $10.3 \%$ were four years $\mathrm{BscN}$ graduates. The study participant had diverse work experience between 1-10 years 59\%; $11-20$ years $10.3 \%$; 21 years \& above $5.1 \%$, while $25.6 \%$ had no work experience/just completed their basic nursing diploma.

Assessment of nurses' knowledge included five questions regarding colostomy care. The question no. 1 about the definition of colostomy in pre-test the mean score was $2.28 \pm 1.40$. These findings are similar to previous study (Bhzeh, et al., 2013). While of the same question in post-test mean score was 4.69 \pm 1.13 , P-value $<0.05$, Paired't' test showed that the nurses gained significant knowledge on definition of the colostomy after the in-service education session. Question no. 2 regarding types of colostomy in pre-test the mean score was $0.46 \pm 0.94$ findings are similar to a study conducted at Assuit University Hospital, Egypt reporting that only 9.6\% of the nurses had true understanding about types of the colostomy (Bhzeh, et al., 2013). Whereas of the same question in post-test mean score was $4.59 \pm 1.35$, P-value $<0.05$. Paired 't' test found the significant enhancement of nurses knowledge about types of colostomy after attending the in-service session. Question no. 3 about the teaching needs of the colostomy patients the mean score in pre-test was $1.36 \pm 1.22$. While the same question in post-test mean score was $4.15 \pm 1.51$, P-value $<0.05$, Paired ' $t$ ' test proved the significant increase in nurses knowledge about the teaching needs of the colostomy patients after receiving the in-service education. Question no. 4 in pre-test regarding the food that should be recommended to colostomy patients showed the mean score of $0.23 \pm 0.49$ and the same question repeated in post-test showed the significant difference in mean score 4.44 \pm 1.37 , P-value $<0.05$. Paired' $t$ ' test observed that the nurses gained noteworthy knowledge about the food that should be recommended to colostomy patients after the intervention of in-service education. The last item question no. 5 in pre-test that was about listing the supplies and 3 competencies required for colostomy care the mean score was $1.74 \pm 1.76$ and in post-test same repeated question illustrated mean score $4.51 \pm 1.17$ and $p$-value $<0.05$, Paired ' $t$ ' test established the significant improvement in nurses knowledge regarding colostomy care after attending the in-service session.

Data was further analyzed and found, that overall pre-test and post-test statistically showed significant difference in the knowledge score obtained by the study participants. The overall pre-test mean score came out to be $6.08 \pm 3.74$ showing that the majority of study participants had poor and average knowledge about the colostomy care. These findings are similar to recent study (Lebona, et al., 2016). Previous literature also reported unsatisfactory level of nurses knowledge regarding colostomy care pointing out that nurses were unable to provide quality nursing care to colostomy patients due to lack of required information related colostomy care. This deficiency in nurses knowledge may be due to lack of information and set standard for colostomy care (Bhzeh, et al., 2013). Present study also presume that study participants coming from different parts of the country 
Texila International Journal of Clinical Research

Volume 3, Issue 2, Dec 2016

with different background of schooling and work experience carrying from low standard organizations and joining the study hospital are deprived from on-job/continue education opportunities. These nurses should be given the top priority to assess their learning need specifically related to colostomy care and provided with in-service education sessions.

Overall post-test showed mean score of $22.38 \pm 5.54$ establishing that the majority of the study participants ( $n=34,87.18 \%)$ had excellent level of knowledge about colostomy care after the inservice education session. Paired't' test revealed that nurses had significant knowledge gain after the in-service education session. P-value $<0.05$, the null hypothesis (H0) rejected at the $95 \%$ confidence interval (CI). Similar findings were found in the literature showing the significant difference between pre-test and post-test scores on nurses knowledge for ostomy care (Bales, 2010).

Many studies suggested to keep the nurses knowledge and skills up dated to help the colostomy patients (Ardigo \& Amante, 2013; Bulkley et al., 2013; Dabirian, et al., 2011; Gül, et al., 2013; Sun et al., 2014). Nurses should be equipped with necessary knowledge and skills to help the colostomy patients. A specialized ostomy nurses are needed for ostomy management and minimizing the complications related to stoma (Zakri, et al., 2010).

This study results corresponds to recent literature that nurses who are up-dated with current knowledge and skills related to colostomy care are in better position to provide comfort to the patients who live with colostomy (Ardigo \& Amante, 2013; Bhzeh, et al., 2013; Bulkley, et al., 2013; Recalla, et al., 2013; Zakri, et al., 2010). Comfort concept is very much important in hospital setting where sick people come to seek comfort in general and colostomy care specifically. Nurses has primary responsibility to assess and listen to the colostomy patients carefully to understand their problem and plan appropriate interventions to relief their pain and worries without wasting time. Nurses are responsible to own the colostomy patients and look after their comfort level: physical, psychological, social and spiritual needs on time as expected by the individuals.

At the end of the in-service education session the study participants verbalized that, they feel happy and empowered with the gained knowledge to practice colostomy care. Nurses showed high confidence and dedication to apply the learnt knowledge at work place. Further, they were eager to learn more about the colostomy care. This indicated that colostomy care in-service education should be on going for nurses to keep them updated with warranted knowledge and skills for colostomy care. The author believes that in-service education sessions for nurses are always highly beneficial for their professional practice, delighted patients and improving the image of the organization in which they work. It is prime important for nurse managers to assess/reassess this essential aspect of nurse's knowledge time to time, so, they may be provided with in-service education/workshops/seminars on regular basis.

\section{Limitations}

Limitations of the current study were small sample size and short tool for data collection. Although this study plan limits the generalization of the findings, the information provided supports the need for further research on a larger sample of the nurses caring for colostomy patients. A much larger study sample will validate the reliability and confirming the findings of this study.

\section{Conclusion}

In conclusion, the pre-test statistically established that the study participants were deficient of knowledge regarding colostomy care. However, the post-test statistically proved that structured education program was highly effective to improve the knowledge towards colostomy care among the nurses working in male and female surgical units showing significant difference in pre-test and posttest scores. At the end of the session the study participants verbalized that, they feel happy and empowered with the gained knowledge to practice colostomy care. They also showed high interest to learn more about the topic. Therefore, it is prime important for nurse managers to assess/reassess this essential aspect of nurse's knowledge so, nurses may be provided with in-service education/workshops/seminars on regular basis. 
Although the sample size was small and study findings cannot be generalized even then study has the implication on nursing practice, education, administration and research.

Further, interventional/experimental research is warranted on larger sample to verify the findings of this study.

\section{Implication of the study \\ Clinical nursing practice}

The study aimed to assess the nurse's knowledge and indicate the need for required knowledge regarding colostomy care. The study will motivate the nurses to provide quality care to the colostomy patients. Moreover, the study will indicate the nurses need for specialized colostomy care modular education requirement. The study will help in reducing the length of hospital stay and cost of the treatment in colostomy patients. Colostomy increases the risk of infection of the surrounding skin and infection of stoma, malnutrition, non compliance of the treatment, complications of the stoma that are stoma retraction, prolapsed, hernia and other psychological problems in patients. Further, colostomy leads to poor quality of life so this research will build up foundation to improve the quality of life of the patients with lifelong colostomy. Recent literature suggest that every organization and profession must set standards of care to guide their workers to deliver best possible care (Bhzeh, et al., 2013).

\section{Nursing education}

The educational needs of colostomy patients are diverse. Nurses need to learn more about preparing the patients for self-care and living and managing with colostomy. Nurses must educate the patients and family members (caregiver) that the risk of infection of the surrounding skin and infection of stoma, malnutrition, complications of the stoma (stoma retraction, prolapsed, hernia), psychological problems and non compliance of treatment can prolong the hospital stay and treatment cost. Patient and family teaching like the need of frequent colostomy care reduce the side effects and complications of colostomy and ultimately the cost of treatment. The nurse educators may use the study findings as useful information for the students. Nursing education should instill the values and sense of accountability in the students to educate the colostomy patient and caregiver for comfortable living with colostomy.

\section{Nursing administration}

The nurse administrator can make use of these study findings to encourage the nurses to practice evidence-based practice and transfer the knowledge among the student nurses. In addition, seminars and workshop can be organized to strengthen the base of knowledge related to colostomy care. The nurse administrators can promote further research on same topic to develop body of knowledge for generalization of the findings and diverse aspects of the research for further inquiry into the problem. The findings of the study should be used as a basis of in-service education programs for nurses so their knowledge regarding colostomy care may be enhanced.

\section{Nursing research}

There is limited research work done on nurse's knowledge regarding colostomy care. The research findings and research design may be used as avenues in future researches for generalization. Nurses working with colostomy patients on bedside should be actively involved in research activities to generate evidence-based knowledge with the support of senior nurses/managers.

\section{Acknowledgement}

My exceptional thanks to Ghuncha Samee Nurse Manager to identify the nurses training need and involve Nursing Education Services Department to facilitate in-service session for nurse's professional development. 
Texila International Journal of Clinical Research

Volume 3, Issue 2, Dec 2016

My special thanks to Nazish Kashif Clinical instructor to assist the training session from start to end.

I am also thankful to Adnan Zafar office coordinator for his technical support in computer applications and data analysis.

\section{References}

[1]. Anaraki, F., Vafaie, M., Behboo, R., Maghsoodi, N., Esmaeilpour, S., \& Safaee, A. (2012). Quality of life outcomes in patients living with stoma. Indian Journal of Palliative Care, 18(3), 176-180. doi: 10.4103/09731075.105687

[2]. Ardigo, F. S., \& Amante, L. N. (2013). Knowledge of the Professional about Nursing Care of People With ostomies and their Families. Text Context Nursing, 22(4), 1064-1071. doi: https://dx.doi.org/10.1590/S010407072013000400024

[3]. Baldwin, C. M., Grant, M., Wendel, C., Hornbrook, M. C., Herrinton, L. J., McMullen, C., \& Krouse, R. S. (2009). Gender differences in sleep disruption and fatigue on quality of life among persons with ostomies. J Clin Sleep Med, 5(4), 335-343.

[4]. Bales, I. (2010). Testing a computer-based ostomy care training resource for staff nurses. Ostomy Wound Manage, 56(5), 60-69.

[5]. Bhzeh, N. M., Teleb, S. M., Mahmoud, M. A., \& Soliman, A. M. (2013). Colostomy: Developing Nursing Care Standards for Patient with Colostomy. The Medical Journal of Cairo University, 81(2), 57-64.

[6]. Bulkley, J., McMullen, C. K., Hornbrook, M. C., Grant, M., Altschuler, A., Wendel, C. S., \& Krouse, R. S. (2013). Spiritual well-being in long-term colorectal cancer survivors with ostomies. Psychooncology, 22(11), 2513-2521. doi: 10.1002/pon.3318

[7]. Burch, J. (2011). Resuming a normal life: holistic care of the person with an ostomy. British Journal of Community Nursing, 16(8), 366-373. doi: 10.12968/bjcn.2011.16.8.366

[8]. Dabirian, A., Yaghmaei, F., Rassouli, M., \& Tafreshi, M. Z. (2011). Quality of life in ostomy patients: a qualitative study. Patient preference and adherence, 5, 1-5. doi: 10.2147/ppa.s14508

[9]. Gül, P., Ayhan, A., \& Tevfik, P. (2013). Emotions of gynecologic cancer patients dealing with permanent colostomy: a qualitative interview study. Journal of Cancer Therapy, 4(6), 1060-1067. doi: http://dx.doi.org/10.4236/jct.2013.46120

[10]. Krans, B. (January 8, 2016). What is Colostomy Retrieved June 28, 2016, from http://www.healthline.com/health/colostomy\#Overview1

[11]. Krouse, R. S., Herrinton, L. J., Grant, M., Wendel, C. S., Green, S. B., Mohler, M. J., ... Hornbrook, M. C. (2009). Health-Related Quality of Life Among Long-Term Rectal Cancer Survivors With an Ostomy: Manifestations by Sex. Journal of Clinical Oncology, 27(28), 4664-4670. doi: doi:10.1200/JCO.2008.20.9502

[12]. Lebona, B., Jasmine, E., Lakshmi, K., \& Indira, D. (2016). Assess the knowledge regarding colostomy care among staff nurses and nursing students in $\mathrm{NMCH}$, Nellore International Journal of Applied Research, 2(5), 306-310.

[13]. Polit, D. F., \& Beck, C. T. (2004). Nursing research principles and methods (Seventh ed.). Philadelphia: Lippincott Williams \& Wilkins.

[14]. Recalla, S., English, K., Nazarali, R., Mayo, S., Miller, D., \& Gray, M. (2013). Ostomy care and management: a systematic review. Journal Wound Ostomy Continence Nurses, 40(5), 489-500; quiz E481-482. doi: 10.1097/WON.0b013e3182a219a1

[15]. Sinha, A., Goyal, H., Singh, S., \& Rana, S. P. (2009). Quality of life of ostomates with the selected factors in a selected hospital of delhi with a view to develop guidelines for the health professionals. Indian journal of palliative care, 15(2), 111-114. doi: 10.4103/0973-1075.58455

[16]. Smith, D. M., Loewenstein, G., Rozin, P., Sherriff, R. L., \& Ubel, P. A. (2007). Sensitivity to disgust, stigma, and adjustment to life with a colostomy. Journal of research in personality, 41(4), 787-803. doi: 10.1016/j.jrp.2006.09.006 
[17]. Sun, V., Grant, M., McMullen, C. K., Altschuler, A., Mohler, M. J., Hornbrook, M. C., ... Krouse, R. S. (2014). From diagnosis through survivorship: health-care experiences of colorectal cancer survivors with ostomies. Support Care Cancer, 22(6), 1563-1570. doi: 10.1007/s00520-014-2118-2

[18]. Thamilselvam, P., Khairuzi, S., \& Fadzli, I. (2013). Quality of life after colostomy. Reviews of Progress, 1(19), 1-4. doi: http://reviewsofprogress.org/UploadedArticle/37.pdf

[19]. Zakri, R. H., Shah, S. A., Khaliq, T., \& Asghar, T. (2010). Impact of Ostomy Surgery on Hospitalized Patients at a Tertiary Care Hospital in Pakistan. Ann. Pak. Inst. Med. Sci, 6(1), 36-39. 


\title{
Standardization of Herbal Medicine and Enforcement of Regulations in Herbal Medical System
}

\author{
Article by Saveri Raj P.X \\ $P h D$ in Alternative Medicine, Texila American University, India \\ Email: saveriraj106@gmail.com
}

\begin{abstract}
Ever since the birth of mankind there has been a relationship between life, disease, nature and herbal medicinal plants. Primitive medicine men started studying diseases and treatments through the use of nature around him. Through their intuition and by regular practice of trial and error methods, they identified and evolved a system of medical knowledge on herbal remedies. The history of herbal medicine is as old as human history.

In recent decades, there are malpractices, by some unethical herbal practitioners and herbal drug manufactures, which are injurious and at times fatal to the people. Hence the need for standardization of herbal medicine and enforcement of regulations for the same, are indispensable. This Article strives to analyze various measures of standardization, regulations and actions against malpractices in Herbal Medical System to safeguard the welfare of the people.
\end{abstract}

Keywords: Herbal Medical Systems, Standardization, Regulations, People’s welfare.

\section{Introduction}

Herbal medicines have been used since the dawn of civilization to maintain health and to treat various diseases. Seeds, leaves, stems, bark, roots, flowers, and extracts of medicinal plants have been used in herbal drugs for more than two millennia. Herbal drugs are the oldest form of health care known to mankind1. This knowledge on medical practices and traditions were transferred by word of mouth to their generations as folk medicines, and subsequently they have been recorded. Such records are available from ancient countries like India, China, Egypt and Greece. These authentic records demonstrate that the natural medicinal plants have been the main source of healing till the dawn of modern medicine. Herbal medicine system has enabled the world to survive thus far in the history of mankind. Herbal drug is a chief element in various traditional medical systems and a common component in ayurveda, siddha, naturopathy, homeopathy and other such systems2. Herbs are generally considered as safe since they belong to natural sources $\mathbf{3}$.

But now the herbal medical system is facing problems from unethical herbal drug manufacturers and unqualified herbal medical practitioners, causing injurious consequences, and sometimes fatal to the people. And many spurious herbal drugs are made available in the market by some unscrupulous persons, which cause adverse effects to the people who consume them without prescription. And there are some unethical and unqualified herbal practitioners, who cause harm to the people by dealing with insecure herbal medicines.

Therefore, there is an absolute need for standardization and regulations by the Governments concerned. The purpose of this study is to analyze the difficulties involved in the standardization in herbals medicine, and to find suitable measures for the same. Similarly, this study is to analyze the challenges towards regulations in the manufacture and distribution of herbal medicine, and how effectively they can be implemented by the Governments concerned.

\section{Method}

The method adopted for this study is a meta-analysis type of qualitative research, involving perusal of mostly published academic journals on the subject. This study was undertaken from the various 
Texila International Journal of Clinical Research

Volume 3, Issue 2, Dec 2016

resources and sites such as Pub Med, psu.edu, currentscience.ac.in, researchgate.net, caregate.net, scribed etc. with a purpose to analyze the various measures for standardization and regulations of herbal medicine.

\section{Findings of the study}

Herbal Medical System is facing challenges from within its system. There are some unqualified herbal medicine practitioners, unethical herbal medicine manufacturers. There is lack of cooperation among the Herbal doctors with 5 years of education in Herbal Medical System and the Herbal medicine healers without such qualification but have the traditional experience derived from their heirs.

There are adulterators mixing or substituting the original drug material with other spurious, inferior, defective, spoiled, useless other parts of same or different plant or harmful substances or drug which do not confirm with the official standards. Gross substitution with plant material and substitution with exhausted drugs are done in herbal medicine by some unethical herbal doctors.

Deterioration happens especially during storage, leading to the loss of the active ingredients, and the actual medicinal qualities. Physical factors such as air (oxygen), humidity, light, and temperature can bring about deterioration directly or indirectly. There are problems of incorrect storage of herbal medicines. Preservation and dosage measurement are serious problems in developing countries. The label claim and other information provided for the use of a herbal preparation may be far from what is in the container.

Some unethical and unqualified people practice herbal treatments which are injurious and fatal to the health of the patients. They engage in gross substitution of plant material, and deal with exhausted drugs

There is complex nature of herbal products and their formulation for therapeutic use. There are challenges in accurate identification of medicinal herbs; collection of authentic and genuine organic medicinal herbs; the diverse and numerous and range of herbs; constraints with clinical trials with people available for research. The preparations of herbal drugs are either as single herb or as collections of herbs in composite formulae4. This may be the main reason why quality control of oriental herbal drugs is more difficult than that of western drug. Patients use herbal drugs arbitrarily with modern medicines, as there are potential of drug interactions and increased risk of adverse drug reactions. The folkloric use of crude drugs is often empirical and is based on observation from clinical trials without experimental support. Use of heavy metals is permitted in traditional medicines but in specific concentrations, which were mentioned by ancient physicians. There are now many examples of the toxicity caused by the use of heavy metals in the preparations of traditional drugs. Lead, copper, mercury, arsenic, silver and gold that are commonly added to these preparations, have caused toxicity on many occasions.

\section{Discussion on the findings of the study}

It has to be ensured that all herbal medicines is 'evidence based' and validated by scientific research, to ensure safety and efficacy for the cure of a disease, and at the same time with least harm to the patients5. In adhering to these rulings, we have to ensure several key issues like damages and side effects caused by these medicines to the patients. Only evidence based and research based facts on the herbal medicines can safeguard the interests of the people. Therefore, it is imperative that herbal medicine should be subjected to evidence based medical system validated by scientific research.

Many research studies have been done in this field to find out which medicine plant is suitable for which disease; to study which medicinal plant is harmful to humans; nutritional values of medicinal plants; which plant has toxins harmful to humans and so on. Allocation of Government funds for research purposes has to ensure more and more evidence-based scientifically proven herbal medicine.

The preparations are either as single herbs or as collections of herbs in composite formulae. This may be the main reason why quality control of oriental herbal drugs is more difficult than that of western drug. These constraints and challenges have to be resolved to make the herbal medicine a highly effective and beneficial in local and global level.

Potential contaminants of herbal medicines include microorganisms, microbial toxins, pesticides, 
fumigation agents, radioactivity, and the presence of toxic compounds of toxic metals, which are given in the table below, and for which regulations have to be implemented by the governments concerned. In assessing toxicity of an herbal medicine, the dose chosen is very important.

Table: Potential contaminants which require regulations and monitoring in

\begin{tabular}{|l|l|l|}
\hline Si. No & $\begin{array}{l}\text { Type of } \\
\text { contaminant }\end{array}$ & Examples \\
\hline 1 & Microorganisms & $\begin{array}{l}\text { Staphylococcus aureus, } \\
\text { Escherichia coli (certain } \\
\text { strains),Salmonella, } \\
\text { Shigella, etc }\end{array}$ \\
\hline 2 & Microbial toxins & $\begin{array}{l}\text { Tabtoxin from } \\
\text { Pseudomonas syringae, } \\
\text { and trichothecenes from } \\
\text { Fusarium and other fungi } \\
\text { etc }\end{array}$ \\
\hline 3 & Pesticides & $\begin{array}{l}\text { Chlorinated pesticides, } \\
\text { phosphates, carbonate } \\
\text { insecticides, fungicides, } \\
\text { triazine herbicide, } \\
\text { naphthol etc }\end{array}$ \\
\hline 4 & Fumigation agents & $\begin{array}{l}\text { Ethylene oxide, methyl } \\
\text { bromide, etc }\end{array}$ \\
\hline 5 & Radioactivity & $\begin{array}{l}\text { Cs-134, Cs-137, Ru-103, } \\
\text { I-131, Sr-90 etc }\end{array}$ \\
\hline 6 & Metals & $\begin{array}{l}\text { Lead, cadmium, mercury, } \\
\text { arsenic etc }\end{array}$ \\
\hline
\end{tabular}

\section{The quality control of herbal medicine}

The tool primarily used to detect and quantify the elements in most toxic metal analyses is based on atomic absorption spectrometry (AAS). Currently, there have been a number of instruments developed based on the same principle, such as inductively coupled plasma-optical emission spectrometry (ICP-OES). Detection and quantification based on mass spectrometry has also been available using inductively coupled plasma-mass spectrometry (ICP-MS).

The herbs which have toxicity and have no clear evidence should be either restricted to use or banned entirely6. The herbal medicine system faces the complexity in standardizations. There are constraints with clinical trials with people available research. The herbal technology should strive for all aspects of innovation in them to improve the treatment regimens to be more cost effective and to be more patient friendly7. Recent surveys reported in the American news media indicated that a large percentage of the public would like to see products supported by science and by clinical research.

Standardization is a tool in the quality control process. Standardization means adjusting the herbal drug preparation to a defined content of a constituent or group of substances with known therapeutic activity. Standardization of herbal medicines is the process of prescribing a set of standards or inherent characteristics, constant parameters, definitive qualitative and quantitative values that carry an assurance of quality, efficacy, safety and reproducibility. It is the process of developing and agreeing upon technical standards. Standardization is the first step for the establishment of a consistent biological activity, a consistent chemical profile, or simply a quality assurance program for production and manufacturing. The lawful situation of herbal drugs varies from country to country. Developing countries have folk knowledge of herbs and their use in traditional medicine is wide spread8. 
Texila International Journal of Clinical Research

Volume 3, Issue 2, Dec 2016

\section{Conclusion}

Herbal Technology is a scientific exploration on the possible applications for conversion of medicinal plants into products for health, food and cosmetic products. Herbal Technology provides basic knowledge, applied expertise and professional skills for production of herbal medicine, food supplements and health care products. Herbal drug technology is used for converting botanical materials into medicines, where standardization and quality control with proper integration of modern scientific techniques and traditional knowledge is vital.

Clinical research is a scientific inquiry in to the aspects of health and illness in people. It is an analysis of the ways and means to prevent, diagnose and treat illness. Clinical research is a branch of healthcare science that determines the safety and effectiveness of medical devices, diagnostic tools, and 'diagnosis and treatment' of disease. Clinical research studies explore whether a medical strategy, treatment, or device is safe and effective for living beings, especially for humans.

A major factor impeding the development of the medicinal plant based industries in developing countries has been the inadequate measures standardization and regulations in the industrial utilization of medicinal plants.

It is the cardinal responsibility of the regulatory authorities to ensure that consumers get the medication with guarantees purity, safety, potency and efficacy. The regulatory authorities have to rigidly follow various standards of quality prescribed for raw materials and finished products in pharmacopoeias, formularies and manufacturing operation through statutory imposed good manufacturing practices.

Control should be exercised from the process from the beginning to the end and quality assurance along the complete process chain. Government regulations are necessary for the protection of the consumers. It may help to ensure herbal medical system has a long and healthy future. The regulations on correct medicinal plants, avoidance of adulteration and contamination, management of quality of medicinal plant products and derivatives will boost the herbal medicine system.

Stability testing of herbal drugs is a challenging risk, because the entire herb or herbal product is regarded as the active matter, regardless of whether constituents with defined therapeutic activity are known. The purpose of a stability testing is to provide proof on how the quality of the herbal products varies with the time under the influence of environmental factors such as temperature, light, oxygen, moisture, other ingredient in the dosage form, particle size of drug, microbial contamination, trace metal contamination, leaching from the container and to establish a recommended storage condition and shelf-life. Stability testing is necessary to ensure that the product is of satisfactory quality throughout its entire storage period. Stability data can also be generated under accelerated atmospheric conditions of temperature, humidity and light, which is referred to as short term stability and the data so obtained is used for predicting shelf-life of the product.

For herbal drugs and products, standardization should encompass the entire field of study from cultivation of medicinal plant to its clinical application. Standardization of methods and quality control data on safety and efficacy are required for uplifting the status and safety of herbal drugs.

Rigid requirements to ensure the safety, efficacy and quality of herbal medicine have to be enforced. Herbal medicines should acquire scientific and clinical evidence before the release to the market.

In order to assure a consistent and acceptable quality herbal product, care should be taken right from the identification and authentication of herbal raw materials to the verification process of final product9.

The government may issue directions to the local authority on matters relating to health policy, planning for health facilities, standards to be maintained, mode of administration and it shall be the duty of the local authority to carry them out10.

World Health Organization (WHO) has distinct herbal drugs as complete, labeled medicinal products that have vigorous ingredients, aerial or secretive parts of the plant or other plant material or combinations11. World Health Organization has set precise guidelines for the evaluation of the 
safety, efficacy, and quality of herbal medicines12.

In order to achieve scientific and clinical validation of botanicals and herbal preparations, there is a need to adopt approaches using chemical standardization, biological assays, animal models, and clinical trials. Existing technologies are not adequate for complete analyses of constituents. In most developing countries, the costs of analyses and standardization are still too high, especially for small manufacturers. Furthermore, the non-availability of instruments and infrastructure, expertise, and human resources has hampered progress in standardization technology. A new paradigm on the concept of standardization and therapeutic validation of herbal medicines may be required to address the issues. The major challenge to the scientific community is, therefore, to formulate a simple, affordable, and reliable standardization method or protocol to be used in the standardization of herbal products.

According to Willard, standardizing should involve the compilation of complete data on herbs such as the seasons in which the herb is harvested, the ripeness and the taste, smell, appearance, drying, storage, processing and fingerprinting which needs much larger spectrum of five or more active or market constituents 13. Standardization is also considered as the way to deal with the regulations framed by regulatory authorities that require drug measurability and the active ingredients to be stated as product labels14.

The completely finished product quality control testing will lead to the production of standardized and therapeutically effective herbal formulations. This can be achieved only if the herbal medicines are evaluated and analyzed using sophisticated modern techniques of standardization15.

In order to assure a consistent and acceptable quality herbal product, care should be taken right from the identification and authentication of herbal raw materials to the verification process of final product. The following standardization measures are recommended.

Table 2. Standardization Measures in herbals medicine, in the various stages

\begin{tabular}{|c|c|c|}
\hline Sl.No & Stage & Standardization Measures \\
\hline 1 & $\begin{array}{l}\text { Identification and } \\
\text { Authentication }\end{array}$ & $\begin{array}{l}\text { The first check is for the proper } \\
\text { identification of the plant species or } \\
\text { botanical verification by the currently } \\
\text { accepted Latin Binomial name and } \\
\text { synonyms16. It should be checked whether } \\
\text { proper records are maintained for stage of } \\
\text { collection, parts of the plant collected, } \\
\text { regional status botanical identity. }\end{array}$ \\
\hline 2 & Physical tests & $\begin{array}{l}\text { The second check is physical tests which } \\
\text { include organoleptic evaluation (sensory } \\
\text { characters such as taste, appearance, odor, } \\
\text { feel of the drug, etc.), viscosity, moisture } \\
\text { content, pH, disintegration time, } \\
\text { friability, hardness, flow ability, } \\
\text { sedimentation, and ash value. }\end{array}$ \\
\hline 3 & $\begin{array}{l}\text { Chromatographic } \\
\text { and spectroscopic } \\
\text { evaluation }\end{array}$ & $\begin{array}{l}\text { The marker compounds present in the } \\
\text { crude drug or herbal products have to be } \\
\text { verified. Markers play an important role } \\
\text { in fingerprinting of herbs. Quality of drug } \\
\text { can also be assessed by chromatographic } \\
\text { fingerprint. }\end{array}$ \\
\hline 4 & $\begin{array}{l}\text { Microbiological } \\
\text { parameters }\end{array}$ & $\begin{array}{l}\text { Elimination of microbiological } \\
\text { contamination measured as per the } \\
\text { methods described in the Romanian } \\
\text { Pharmacopoeia17, in the British }\end{array}$ \\
\hline
\end{tabular}


Texila International Journal of Clinical Research

Volume 3, Issue 2, Dec 2016

\begin{tabular}{|l|l|l|}
\hline & & Pharmacopoeia 18. \\
\hline 5 & Pesticide check & $\begin{array}{l}\text { Standard limits of pesticides have been set } \\
\text { by WHO and FAO (Food and } \\
\text { Agricultural Organization). The } \\
\text { pesticides should be checked. }\end{array}$ \\
\hline 6 & $\begin{array}{l}\text { Heavy metals } \\
\text { check }\end{array}$ & $\begin{array}{l}\text { Checking for the toxic metals such as Cu, } \\
\text { Zn, Mn, Fe, Pb and Hg19. Standardized } \\
\text { parameters like detection of heavy metals } \\
\text { such as lead, mercury, calcium and } \\
\text { pesticide residues should be checked in } \\
\text { crude drug sample. }\end{array}$ \\
\hline 7 & $\begin{array}{l}\text { Manufacture } \\
\text { stage }\end{array}$ & $\begin{array}{l}\text { The composition of any solvent or solvent } \\
\text { mixture used and the physical state of the } \\
\text { extract should be identified. The nature } \\
\text { and concentration of the solvent and the } \\
\text { physical state of the extract should be } \\
\text { verifiedzo. }\end{array}$ \\
\hline 8 & $\begin{array}{l}\text { Packaging and } \\
\text { storing stage }\end{array}$ & $\begin{array}{l}\text { All packaging materials like bottles and } \\
\text { other packaging materials should be } \\
\text { stored properly. }\end{array}$ \\
\hline 9 & $\begin{array}{l}\text { Ensure good manufacturing practices for } \\
\text { herbal medicines. }\end{array}$ \\
\hline 10 & $\begin{array}{l}\text { Herbal materials may contain } \\
\text { Sanitation } \\
\text { microbiological contaminants. And } \\
\text { during the course of harvesting and } \\
\text { processing herbal materials, herbal } \\
\text { products are prone to produce } \\
\text { microbiological contamination. Therefore } \\
\text { A high level of sanitation and hygiene } \\
\text { during manufacture is necessary }\end{array}$ \\
\hline
\end{tabular}

Solutions suggested for standardization of herbal medicine

There is a compelling need for standardization of Herbal Medical System, to safeguard people's welfare. Actions for obtaining the evidences, required for validation of traditional herbal medicinal products, should be augmented. A rigid application of Herbal Technology measures in Clinical Research is a vital requirement to uplift Herbal Medical System, to be more effectual and operative health care system. All herbal medicines should be subjected to 'evidence based' and validated by scientific research, to ensure safety and efficacy for the cure of a disease, and at the same time with least harm and side-effects to the patients. Many more research studies are required to find out the medicinal and nutritional values of herbs and which are harmful to humans.

Government allocation of funding for research purposes has to ensure that more and more evidence-based scientifically proved herbal products are available for the healthcare system of the people. The herbal medicines have to be evaluated and analyzed using sophisticated modern techniques of standardization. Research studies are required to be done on the effectiveness of medicinal plants for curing diseases. Specific standard should be assigned for experimentation and observation, which would lead to the process of prescribing a set of characteristics for each herbal medicine. The indigenous knowledge on the traditional medical practices and claims must be validated and documented about the ingredients, active constituents, and made accessible to the consumer. 


\section{Solutions suggested for regulation of herbal medicine}

The quality of all herbal medicinal products at all levels has to be ensured by rule of law. There should be stringent action on any malpractice on herbal system. The quality of the herbal products has to undergo stringent regulatory control, if herbal medicines are to assume a respected place in the contemporary health care system. Actions should be enhanced for obtaining the evidences required for validation of traditional herbal medicinal products with scientific proof of its efficacy. The completely finished products should be subjected to quality control testing for all herbal medicines. The quality of the herbal products has to undergo stringent regulatory control, if herbal medicines are to assume a respected place in the contemporary health care system. Factual labeling practice should adequately address quality aspects. Government regulations should ensure to conduct clinical researches to scientifically authenticate the herbal products.

Stability studies should be performed on at least three production batches of the herbal products for the proposed shelf-life, which is normally denoted as long term stability and is performed under natural atmospheric conditions. Stability testing should be conducted on the dosage form packaged in the container closure system proposed for marketing. Governments must update the databases, containing reports of investigations and clinical studies on herbal technology and herbal medicine.

These measures for standardization and the regulatory policies can effectively protect the citizens from the identified problems. When all these issues are implemented potential prospects exist for widespread use of herbal medicine as a safe, effective, and affordable form of healthcare. The above measures of standardization and of Herbal Medicines and enforcement of regulations are bound to ensure the Herbal Medical System, to serve and strive towards perfection and benefit of the humanity worldwide.

\section{References}

[1]. Abhishek, K; Ashutosh, M and Sinha, BN (2006), "Herbal drugs- present status and efforts to promote and regulate cultivation”, The Pharma Review, 6, 73-77.

[2]. Abhishek, K; Ashutosh, $\mathrm{M}$ and Sinha, BN, "Herbal drugs- present status and efforts to promote and regulate cultivation”, Pharma Review, 2006 6, 73-77.

[3]. Bodhisattwa Maiti et al., "Recent Trends in Herbal Drugs: A Review", International Journal of Drug Research and Technology, 2011,Vol.1 (1),17-25

[4]. British Pharmacopoeia, Vol. IV, Appendix XVI B, A356-A363 (2005).

[5]. Good Manufacturing Practices for pharmaceutical products, In: WHO Expert Committee on Specifications for Pharmaceutical Preparations. Thirty-second report. Geneva, World Health Organization, 1992:14-59 (WHO Technical Report Series, No. 823).

[6]. H. Wagner, et al, "Handbook of Medicinal Plants”, Chapter 21, Haworth Medical Press, Binghamton (2001).

[7]. Harish, P (2001), "Herbal drugs", Current Science, 81(1), 15

[8]. M.Mosihuzzaman and M. Iqbal Choudhary, "Protocols On Safety, Efficacy, Standardization, And Documentation Of Herbal Medicine”, Pure Applied. Chemistry, Vol. 80, No. 10, Pp. 2195-2230, 2008. Doi:10. (IUPAC Technical Report)

[9]. Page LR, “Whole Herbs or Standardized Plant Constituents?”, Total Health Journal, 2001,23(4):20.

[10]. Report by David R. and Walker, B, "Regulation of Herbal Medicines and Practitioners”, March 2015.

[11]. Romanian Pharmacopoeia X, Chap. IX, Ed, Medicala, Bucuresti (1993).

[12]. Satarupa Banerjee and Analava Mitra, "Changing Landscape of Herbal Medicine: Technology Attributing Renaissance”, International Journal of Pharmacy and Pharmaceutical Sciences, Vol.4,Supl.1, 2012

[13]. Sukhdev, S; Arun, N and Kalia, AN (2008), "Patentability of herbal products: A review", The Pharma Review, 4,118-124.

[14]. The Madras Public Health Act 1939 \& The Travancore-Cochin Public Health Act 1955.

[15]. Vardana Garg et al., "Facts about Standardization of Herbal Medicine: A Review", Journal of Chinese Integrative Medicine, October 2012, Vol.10, No.10.

[16]. WHO technical report series, "Guidelines for the Assessment of Herbal Medicines”, 1996, 863, 178-184. 
Texila International Journal of Clinical Research

Volume 3, Issue 2, Dec 2016

[17]. WHO Global Survey, "National Policy on Traditional Medicine and Regulation of Herbal Medicines", World Health Organization Geneva, May 2005, 168 pages.

[18]. Willard T, "Edible and Medicinal Plants of the Rocky Mountains”, Calgary, Wild Rose College of Natural Healing,1996.

[19]. WHO guidelines on safety monitoring of herbal medicines in pharmacovigilance systems, World Health Organization, Geneva, 2004

[20]. Winslow, L; Kroll, DJ (1998), “Herbs as Medicines, Archives of Internal Medicine”, 158, 2192-2199. 


\title{
The Prevalence of Needle Stick/Sharp Objects Injury in Hospital Staff and Preventive Practices Taken into Consideration
}

\author{
Article by Badmus Omobolanle Tawakalit \\ Master of Public Health, Texila American University, Nigeria \\ E-mail:b4_badmus@yahoo.com
}

\begin{abstract}
Health care workers are at an increased risk of accidental needle stick injuries because of the environment in which they work.

Objective is to study the prevalence of needle stick injury in the hospital and preventive practices taken into consideration.

Cross-sectional study design used, in a secondary care hospital in kuje, Abuja, Nigeria. Participants were 161 doctors, nurses, lab technicians and hospital cleaners. Statistical analysis: chisquare test and proportions

High percentages (79.5\%) of health care workers were reported to have had one or more needle stick injuries in their career. The average number of the injuries ever found was like 3.85\% per health care workers. 36 (22.4\%) reported to have had needle stick injuries in the last one month. More than half claimed it was due to tiredness that caused the injury. Most of the injuries occurred during recapping, most washed the site of injury with soap and water while few did nothing. Only 10 took post exposure prophylaxis of HIV/AIDS after their injury.

In conclusion occurrence of needle stick injuries were found to be common. Recapping of needles which is an avoidable practice was found to be part of the contributing factor to the injuries.

Therefore prevention of needle stick injuries is an integral part of preventive programs and training and retraining of hospital staff for safety practices in handling needles and sharp objects needs to be continuous in the hospital.
\end{abstract}

\section{Introduction}

Needle stick injuries are injuries caused by accidental needle puncture into the skin. This is a form of occupational hazard for people who work with hypodermic syringes and other needle equipment. The injuries can occur at any time when people use, disassemble or dispose needles. Needle stick injuries also known as percutaneous injuries or percutaneous exposure incidence to a sharp object or needles penetrating into the skin ,the sharp object or needles which was in contact with blood, body fluid or tissue of a client or patient.

Other sharp objects other than needles used in the hospital settings include items like scalpels, lancets, razor blades, scissors, metal wires, pins, cutters and staples.

When all these sharp objects are not disposed properly, they can become concealed in the garbage or linen causing injury to workers who encounter them unexpectedly.

Accidental punctures by these contaminated sharp objects can inject hazardous fluids into the body.

The major blood-borne pathogens that are of concern in needle stick injury are Hepatitis B virus, Hepatitis C virus and HIV, however there are some other infectious agents which could be bacteria or fungi that also have potential to transmit through needle injuries, few of which are syphilis, toxoplasmosis, diphtheria, brucellosis etc.

Injuries can occur at any stage of using, assembling, disassembling or disposing the items. It could when trying to access an intravenous line or when taking sample for investigations or during insertion or removal of needle. This can also occur during clean up that affect the cleaners most often when left in an unusual location. It is also common during surgical procedures when there is collision with sharps or co-workers, or during transfer of equipment or when suturing. 
Texila International Journal of Clinical Research

Volume 3, Issue 2, Dec 2016

So the nature of the procedure, staff experience in handling sharps and disposal of this sharps are all factors that influences these occurrences.

Preventing these injuries should be a paramount concern in health policy and is the most effective way to protect works.

\section{Objective}

Promoting the use of preventive practices in preventing occupational health injury from needlestick and sharps in the hospital settings.

\section{Methodology}

A comprehensive sharps injury prevention program conducted to recommend guidelines, effective disposal system, employee training and safe recapping procedures.

\section{What is occupational health hazards?}

Occupational health hazards are occupational illnesses that results from exposure in the workplace to physical, chemical or biological agents to the extent that the health of the worker is affected .In the case of the occupational health hazards in the hospitals, there are various hazards which the health workers are exposed to, few of which include accident (explosion, fires, needle sticks and sharps injuries), radiation, exposure to noxious chemicals and even assaults.

There are areas of special exposure to health hazards in the health services, which increases the risk of health hazards; these areas include accident and emergency services, ambulance care services, mental health institution, the prison services, care of elderly or attending to aggressive patient.

Also some activities increases risk of hazard in health works. Activities such as taking specimen of blood, body fluid, surgical procedures, dressing or treatment of wound, handling sharp instrument and handling health care waste.

Other physical and environmental hazards common in the hospital are slippery floors, noise, poor lighting, and inadequate ventilation. ${ }^{5}$

Blood happens to be the body fluid that presents the greatest risk of infection to health care workers.

Blood borne pathogens happen to be the microorganisms that transmit diseases by contact with blood. Contact could be by direct such as needle sticks or splash, or indirect as at when the mucous membrane comes in contact with a contaminated blood.

The most common conditions are Hepatitis C and B and Human Immunodeficiency virus (HVS).

\section{What are the hazards of needle stick and sharps injuries}

Needle sticks and other sharps injuries continue to be a significant hazard in the hospital settings, which exposes workers to blood borne pathogens.

It is estimated that millions of healthcare workers and related occupations are at risk of occupational exposure to blood borne pathogens.

According to the Center for Disease Control and Prevention (CDC), about 385,000 sharps injuries occur annually in hospital workers. ${ }^{6}$

Blood borne pathogens: are pathogenic microorganisms present in the human blood that can cause diseases in human. Examples of these pathogens include Human Immunodeficiency Virus (HIV), Hepatitis C (HCV), Hepatitis B Virus (HBV) and others.

The exposure of human blood to these pathogens is mostly because of unsafe needle devices, improper handling and disposal of needles and other sharps.

Contaminated Sharps are any contaminated object that can penetrate the skin these includes but not limited to needles, scalpels, broken glass, broken capillary tubes and exposure to dental wires. Exposure could also be from used disposable razors that could be contaminated with blood or I.V connector system that uses needles to connect I.V. setups. 


\section{How can the injuries be prevented}

In preventing needle stick injuries in the health care system there are some processes for selecting and evaluating needle devices with safety features, this processes include:

Forming a multidisciplinary team that will include workers to develop, implement and evaluate a plan to reduce the occurrence of the needle stick injuries in the institution.

Identifying priorities based on the assessment of how the needle stick injuries occurs, the patterns of device used. Settling the needle device with safety features.

When selecting a safe device, it's important to identify its intended scope of use in the health facility. Conducting a product evaluation.

Training and retraining of staff in the correct use of the devices

Monitor the use of new devices, after it is introduced to determine if additional training is needed.

Practicing standard practices, in work practice control in minimizing exposure to blood borne pathogens

Personal protective equipment

Proper handling and contamination of sharps.

Using safe needle container near areas needles are often found and used

Making vaccines and vaccination series of hepatitis B vaccines available for all employees

Post exposure evaluation and follow up.

Syringes: used syringes with needle stick features should never be recapped and they should be disposed in sharps containers after used or it could be destroyed using electrical equipment.

Sharp containers: must be provided in all areas where sharps are used. They must be closable, must resist puncture, leak proof and free from contaminant. This should be changed when it's about three quarterly full. It should be easy to access.

Blood collection: injuries from blood collection can be reduced by the use of needle shields, selfblunting needles, hinged needle shield or butterfly needles.

Sutures needles: sutures needles with blunt tips reduces the risk of needle sticks injuries by $69 \%$ and are recommended to reduce percutaneous injuries ${ }^{7}$.

PPE: gloves, gowns and eye and face should be protected, in adequate quantities and at no cost to the employers, that's depending on the part of the body that is exposed.

Vaccination and Treatment: workers at risk of blood borne exposure should be offered HBV vaccines, since this can protect from infection. In the case of HIV exposure, post exposure prophylaxis should be made available within two hours of exposure.

Surveillance Program: this program should provide in-depth analysis of accidents and are important tool for obtaining information, such program's goals are to determine the rate of injuries, investigating the factors that causes the injuries, ensuring workers that are injured gets proper treatments, identifying areas where preventive programs needs to be improved ${ }^{1}$.

\section{Occupational safety and hygiene}

Occupational safety and hygiene is a multidisciplinary field that is concerned with the safety, health and welfare of workers.

Health care workers are exposed to many hazards that affect their health and well-being ${ }^{10}$.

Occupational safety and hygiene practices vary in different nations with different legislation, regulations, enforcement and incentives for compliance, some states promotes this by providing public monies as subsides, grants or financing, while others do it by creating tax systems incentives. The US Occupational Safety and Health Administration (OSHA) estimates 5.6 million workers in the healthcare industry are at risk of occupational exposure to blood borne disease via percutaneous injuries $^{9}$

The roles and responsibility of occupational safety and hygiene professionals includes evaluating work environment, developing measures that might prevent injuries and illnesses in the work place, 
providing medical examinations and assessing the success of health workers program. There also provide information on occupational hygiene and safety risks in the work place.

Four certified service providers are required for this profession, this includes;

A safety professional

An occupational hygienist

An occupational physician

A work and organization specialist. ${ }^{9}$

The safety professional should be able to assess risk in the work place. This assessment should include identification of the hazard, identifying all staff affected by these hazards, evaluating the risk and identifying and prioritizing appropriate control measures. ${ }^{9}$

\section{Basic findings}

Study conducted among hospital staff of Kuje General Hospital Kuje, Abuja in September 2016.

Responded were 161 health care workers consisting of doctors, nurses and laboratory technicians and hospital cleaners.

About 79.5\% (128) of health care workers have being reported to have had one or more needle sticks injuries in their career.

In an average the number of needle stick injuries ever found was $3.85 \%$ per health care worker $\left(^{+}\right.$ 3.29SD).

For workers who had been working for at least 1 year, the mean number of injuries was as high as 4.5(+_3.4SD), 36(22.4\%) workers was said to have had needle stick injuries in the last 1 month.

These injuries occurred more among the doctors (25.6\%), Lab technicians (24.6\%) and Nurses (20, $5 \%$ ), most of which they didn't know the status of the patients.

Among them 2\% (17.5\%)were said to have had needle stick injuries from high risk patients with HIV, Hepatitis B and C, was more among doctors (21\%) and lab scientist (9.7\%).

Among the 128 who have had needle stick injuries, 35 (27.3\%) were not wearing gloves at the time it occurred.

About 103 (84.8\%) of them accepted that it was caused by self while the remaining $15.6 \%$ said it was someone else. Majority was caused by hollow-bore type of needle (89, 69.5\%) while about 30.5\% were from solid-bore needles. It is believe that hollow-bore needles are more efficient in transmitting bloodborne infection than solid needles such as suture ${ }^{3,5}$

Regarding time of injury most of the injuries occurred during the use (29.4\%), while greater part was after use, but before disposal $(83,65.5 \%)$ only few $5 \%$ occurred during disposal.

The cause of injury was asked, it was found that more than half was due to Fatigue(50.4\%) about $10.9 \%$ believed the injuries could have being prevented, most occurred during recapping(34.0\%). 37 cases occurred during handling of needles, 27 during collision with another person and 39 due to manipulation of patient.

\begin{tabular}{|lll|}
\hline Causes of the recent needle stick injuries $(\mathrm{n}=128)$ & & \\
Causes of injury & $\mathrm{N}$ & $\%$ \\
Fatigue & 64 & 50.4 \\
Rushed & 16 & 11.7 \\
Lack of assistance & 34 & 27.0 \\
Could have been prevented & 14 & 10.9 \\
\hline
\end{tabular}




\begin{tabular}{|c|c|c|c|c|}
\hline \multicolumn{5}{|c|}{$\begin{array}{l}\text { Responses of staff to different questions on needle stick injure. ( } \mathrm{n}=161 \text { who } \\
\text { had the needle stick injury). }\end{array}$} \\
\hline$\underline{\text { Doctors }}$ & nurses & $\underline{\text { lab }}$ & $\underline{\text { scientists }}$ & cleaners \\
\hline $\begin{array}{l}\text { Proportion who had injury } \\
\text { within the last } 1 \text { month }\end{array}$ & 25.6 & 20.5 & 24.6 & 26.6 \\
\hline $\begin{array}{l}\text { Proportion who had been } \\
\text { wearing gloves }\end{array}$ & 84.3 & 65.5 & 29.4 & 77.6 \\
\hline $\begin{array}{l}\text { proportion who received their } \\
\text { injury during recapping }\end{array}$ & 36.5 & 29.2 & 48.4 & 28.6 \\
\hline $\begin{array}{l}\text { Proportion who reported the } \\
\text { case }\end{array}$ & 38.8 & 6.5 & 23.2 & 7.9 \\
\hline proportion who had & 6.1 & 6.5 & 5.2 & \\
\hline
\end{tabular}

All figures represent percentage (\%) for each category of hospital staff

After injury only26 (20.7\%) gottheir blood tested immediately after the injury and only 35 (27.5\%) reported the cases.

\begin{tabular}{|lll|}
\hline $\begin{array}{l}\text { Table 3 } \\
\text { Responses taken after the needle } \\
\text { stick injury }\end{array}$ & $\mathrm{N}$ & $\%$ \\
Response after the injury & 16 & 14.8 \\
Nothing & 11 & 8.6 \\
Washed hand & 51 & 39.8 \\
With soap and water & 16 & 10.5 \\
Applied spirit & 1 & 0.3 \\
Pep & 17 & 13.7 \\
Water, soap and spirit & 6 & 4.3 \\
Water and spirit only & 8 & 6.3 \\
Water, soap, spirit and pep & \\
$\begin{array}{l}\text { Note: The total does not sum up to } \\
\text { infrequent were not shown }\end{array}$ & \\
\hline
\end{tabular}

\section{Discussion}

A large number of workers have being reported to have had needle stick injuries in their career.

In this study 36 workers among 161 health care workers have had needle stick injuries within the past 1 month, giving a rate of about $3.47 \%$ per annum. Only $27.5 \%$ said to have reported the case. Pournaras et al. had found the incidence of NSI to be $2.4 \%$ per year, but which they themselves discuss to be apparent underreporting as they considered only reported incidents ${ }^{8}$.Study by WHO on global burden of sharp injury estimated the average number of injuries per health care worker in Africa is 2-4 sharps injuries per year on average. ${ }^{7}$

Preventive practices like wearing of gloves is known to be important line of defense, but higher numbers of nurses and lab technicians have not been practicing it.

Training programs should be encouraged and it should be emphasized that need to maintain utmost care and caution in handling needle sticks or sharps are paramount.

About $50.4 \%$ of health care worker who ascribed their injuries to being fatigue, have being found to having long working hours. The hospital system has a hectic and stressful system and due to the long hours of duty, it must be ensured health workers should be given breaks in between work, to refresh themselves up.

Other studies shows recapping as an important cause of $\mathrm{NSI}^{12}$, so training and retraining of staff should be emphasized. 
Texila International Journal of Clinical Research

Volume 3, Issue 2, Dec 2016

Previous studies too have shown a wide difference in the occurrence rate of NSI in the studies which asked directly from the HCWs compared to those relying only on those who self -reported to the institution. ${ }^{11}$

Needle stick injuries are important occupational hazard that health care workers face daily, but can be minimized to a large extent. So prevention of injuries is the best way to prevent several diseases in the health care workers.

It is recommended that government should make policies that would enforce all hospital management to develop a multi-pronged strategy to deal with needle stick injuries, and also promoting health and setting up an adequate surveillance mechanism.

\section{Conclusion}

Occurrence of needle stick injuries was found to be common.

Recapping of needles which is an avoidable practice was found to be part of the contributing factors to the injuries.

Therefore prevention of needle stick injuries is an integral part of preventive

programs and training and retraining of health care works for safety practices in handling needles and sharp objects needs to be continuous in the hospital

\section{References}

[1]. Adeboye AA, Moss GB, Soyinka F, Kreiss Jk. The epidemiology of needlestick and sharp instrument accidents in Nigerian Hospital. Infect Control Hosp Epidemiol 1994; 15:27-31

[2]. British journal of medicine(occupational and environmental medicine);Journal List > Br.J Ind Med > v.44(8).1987 Aug

[3]. CCOHS; Canadian Center for occupational health and safety. https://www.ccohs.ca/oshanswers/diseases/needlestick. Copyright 1997-2016

[4]. Elder A, Paterson c. Sharp injuries in UK health care: A review of injury rates, viral transmission and potential efficacy of safety devices. Occup Med (Lond) 2006;56:566-74

[5]. Ganczak M., Bohatyrewicz A., Korzen M., Karakiewicz B. The comparison of sharp injuries reported by doctors versus nurses from surgical wards in the context of the prevalence of HBV, HCV and HIV infections.

Pol Przegl Chir. 2012;84:190-195.(PubMed)

[6]. Gestal JJ. Occupational hazards in hospital: risk of infection. Br J Ind Med 1987 jul

[7]. Henderson D.K. management of needlestick injuries. JAMA.2012;307:75-84(PubMed)

[8]. Kirchner, B(2012). Safety in ambulatory surgery centers: Occupational safety and Health Administration surveys”. AORN Journal. 96: 540-5.

[9]. OSHA USD of Labour? www.osha,gov/SCTc/etools/hazards

[10]. Occupational health, safety and hygiene- The Carter center. www.cartercenter.org>org>lecture_notes

[11]. Pruss- ustun A, Rapiti E, Hustin Y. sharps injuries; global burden of disease from sharps injuries to health - care workers. Geneva: WHO; 2003(Who Environmental Burden of Disease Series, No.3)

[12]. Pournaras S, Tsakris A, Mandreveli K, Faitatzidou A, Douboyas J, Tourkantonis A. Reported needlestick and sharp injuries among health care workers in Greek general hospital. Occup Med (Lond) 1999;49;423-6 


\title{
Patient's ability to consent during Inpatient Chemotherapy Treatment or Outpatient Radiotherapy Treatment in a tertiary care hospital of New Delhi, India
}

\author{
Article by Kavita Gupta \\ Ph.D. Clinical Research, Texila American University, India \\ E-mail: 16kavitagupta@texilaconnect.com
}

\begin{abstract}
Background: With the increase in the rate of cancer morbidity, there was a marked increase in the research area for the development of strategies for the management of cancer disease. However, this objective could only be achieved through various phase of clinical trials requiring humans as a subject of study. This raised an ethical issue as survey research conducted in the form of questionnaires consisted of the classic questions of anxiety, depression, psychosis, or agitation which may or may not suit the patient's ability to consent due to certain factors. In the present study, it was observed that how the patient and the care-giver supported in the completion of this research survey during the patient's inpatient chemotherapy or outpatient radiotherapy treatment.

Objectives: The primary objective of the present study was to determine and analyze the percentage of cancer patients enrolled in the study that required assistance either from the interviewer or from the care-givers in filling up of the QOL questionnaire form during the inpatient chemotherapy or outpatient radiotherapy sessions.

Methods: It was a cross-sectional, descriptive, hospital based evaluation study. Total duration of the study was 5 months (December 2015- April 2016), conducted in Medical and Radiation Oncology department of DR. B.L. Kapur Memorial Hospital, New Delhi, India. A convenience sample of 60 patients with cancer was selected. They were further divided into Chemotherapy group $(n=30)$ and Radiotherapy group $(n=30)$. Therefore, 30 cancer patients were on chemotherapy and the other 30 cancer patients were on radiotherapy. Data was obtained through direct interview, using validated Psychological intervention tool in the form of Questionnaire: WHOQOL-Bref Questionnaire, Zung Self-Rating Anxiety scale and Zung Self-Rating Depression scale.

Results: A total of 60 cancer patients were included in the study in which Chemotherapy group consisted of 30n cancer patients and Radiotherapy group consisted of 30n cancer patients. In the study, 6(20\%) were males and 24(80\%) were females in the chemotherapy group, and, 15(50\%) were males and $15(50 \%)$ were females in the radiotherapy group. Majority of the patients 32(53.34\%) were in the age range of 46-60 years. It was observed that maximum patients in chemotherapy, as well as, radiotherapy groups marked their ability to consent to participate in the study with less assistance from the researcher and the care-givers even during the treatment regimen.

Conclusion: It could be concluded from the results obtained from the study that the patients undergoing chemotherapy (66.6\%), or radiotherapy (76.7\%) sessions were able to consent freely without much assistance of the researcher or the care-giver. This further implied that in Indian scenario people are becoming more aware and thus, cooperated by participating in research studies so as to help manage the deadly disease "CANCER".
\end{abstract}

Keywords: Cancer, Patient's consent, Patient themselves, Assisted by researcher, Assisted by relatives. 
Texila International Journal of Clinical Research

Volume 3, Issue 2, Dec 2016

$\begin{array}{lll}\text { Abbreviations } & \\ \text { QOL } & : \text { Quality of life } \\ \text { QLQ } & : \text { Quality life Questionnaire } \\ \text { WHOQOL-Bref } & : \text { World Health Organization Quality of life assessment-a short brief version } \\ \text { ZSAS } & : \quad \text { Zung Self-Rating Anxiety scale } \\ \text { ZSDS } & : \text { Zung Self-Rating Depression scale } \\ \text { CT } & : \text { Chemotherapy Treatment } \\ \text { RT } & : \text { Radiotherapy Treatment } \\ \text { DOM } & : \text { Third Party Administrator } \\ \text { TPA } & : \text { Fine needle aspiration cytology } \\ \text { FNAC } & \text { Standard deviation. }\end{array}$

\section{Introduction}

According to the research, it was predicted that cancer incidence in India varied from 44-122 per 100,000 population in males and $52-128$ per 100,000 population in females ${ }^{[1]}$. It was estimated that, at present, nearly one million new cancer cases were being detected annually in the country ${ }^{[1]}$. For patients and their family, a diagnosis of cancer brings challenges to many aspects of daily life, with a major concern of being maintaining the highest quality of life possible during and after the experience ${ }^{[1]}$. Because of the aggressive cancer symptoms, patients with cancer and their families might face ongoing and challenging medical decisions that progressively and rapidly erode cognition ${ }^{[2]}$. Quality of Life (QOL) is a descriptive term that referred to people's emotional, social, and physical well-being as well as their ability to function in ordinary tasks of living ${ }^{[1]}$. The informed consent process consisted of 3 principles, viz., (1) Knowledge (the research proposal should state benefits and risks along with the alternative treatments and the outcome measures; (2) Voluntariness of the participant in which the pressure from the family is acceptable; (3) Competence where patient should understand the information disclosed ${ }^{[3]}$. Assessing a patient's medical decision-making capacity is a part of every medical research study ${ }^{[3]}$. The process is generally spontaneous and straightforward: during the performance of routine inpatient and outpatient visits, the researcher should confirm the ability of the cancer patients to understand their medical condition and options for care ${ }^{[3]}$. To perform this analysis of ability of a cancer patient to consent, a directed clinical interview is the best available option ${ }^{[3]}$. The clinical interview might consist of questions pertaining to case history from the care-givers or laboratory findings ${ }^{[3]}$. For some patients, however, the assessment may not be straightforward ${ }^{[3]}$. Currently, if a person failed to have the capacity to consent to treatment or research, substitute decision makers were employed (e.g., legal representatives or family members) ${ }^{[4]}$. Thus, a judgement by health care professionals that a person was not competent to provide informed consent might have a profound effect on the autonomy, or free will, of an individual ${ }^{[4]}$. Contemporary ideas on informed consent stem, in part, from the Nuremberg Code following the Second World War ${ }^{[4]}$. The code specified that consent to research should be voluntary and that participants should have free choice, adequate understanding, and the capacity to give consent ${ }^{[4]}$. 


\section{Aims}

The main objectives of the present study were:

i. To determine and analyze the ability of cancer patient to consent during inpatient chemotherapy treatment or outpatient radiotherapy treatment.

ii. To evaluate the percentage of cancer patients enrolled in the study that required assistance either from the interviewer or from the care-givers in filling up the QOL questionnaire form during the chemotherapy or radiotherapy sessions.

\section{Patients and methods}

During the conduct of the proposed study Ethical Procedures were respected. After obtaining authorized Ethical approval (Ref. No.: IRB/AARCE/5/DEC/2015/1 and dated December $7^{\text {th }}$, 2015) for the research study protocol from IRB and Ethical committee from Dr. B.L. Kapur Memorial Hospital, New Delhi, patients and their caregivers were approached in the inpatient as well as, outpatient clinic, where the purpose of the study was explained and they were invited to participate. Patients who agreed to participate were asked to sign an Informed Consent Form followed by the implementation of the instrument in the form of questionnaires.

The Research Design of the proposed approved study protocol included the following Sampling Technique:

1. Sample Size: The target population of patients undergoing Chemotherapy and Radiotherapy sessions.

Total participants $=60$ divided in the following pattern:

$\mathrm{N}$ (Chemotherapy treatment) $=30 \mathrm{n}$

$\mathrm{N}$ (Radiotherapy treatment) $=30 \mathrm{n}$

2. Eligibility criteria of the study:

\section{Inclusion criteria}

a. Breast cancer and sub-sites of head and neck tumors (e.g., nasopharyngeal, thyroid cancer, and parotid tumors)

b. Patients aged 18years or older,

c. Clinically diagnosed cases.

d. Cancer diagnosis confirmed by biopsy or FNAC,

e. Undergoing/during the treatment sessions

f. Voluntarily agreed to join the study.

g. Aware of diagnosis and predicted prognosis

\section{Exclusion criteria}

a. Inadequate clinical condition (ambulatory and terminally ill patients) to respond to an interview.

b. Have difficulty in understanding the questionnaire or communicating.

c. Patients who were serious and didn't give consent were excluded from the study.

d. Had a history of psychiatric disorder

e. Choice of chemotherapy drugs and their dosage, irradiation dosage.

3. The study involved primary research by Individual face-to-face interviews with 60 cancer patients undergoing either chemotherapy or radiotherapy treatment sessions through Structured and Validated WHOQOL-Bref Questionnaire that consisted of 26 questions on a five-point likert scale ${ }^{[5]}$, Zung Self Rating Anxiety Scale (ZSAS) that consisted of 20 questions on a fourpoint likert scale ${ }^{[6]}$, and Zung Self-rating Depression Scale (ZSDS) that consisted of 20 questions on a four-point likert scale ${ }^{[7]}$.

4. Eligible patients were identified through an institutional database or by referring physicians and were approached at their simulation appointment. After giving written informed consent from 
the patient or their caregivers, participants completed the assessment that included the self-report measures.

5. Patients who agreed to participate were asked to sign an informed consent and then the instruments in the form of questionnaire were applied. Subjective areas were covered using case histories.

6. Special care of the potential risks due to emotional distress was taken care of so that their dignity was not harmed.

7. A total of 5-6 interviews were performed per week, in the time and days most suitable for the clinic in the months of December 2015 to April 2016.

8. Each interview lasted approximately 25-60 minutes and all patients were thanked for their participation, valuable time and information in the end.

\section{Results}

\section{(a) Data collection}

Treatment-related symptoms were assessed using a series of interviews through standard questionnaires of WHOQOL-Bref, the core questionnaire, followed by Zung Self-rating Anxiety Scale (ZSAS) and Zung Self Rating Depression Scale (ZSDS) Questionnaires. The questionnaire was provided in a language that the patient could understand (English / Hindi) followed by interview of the patient who was either undergoing chemotherapy or radiotherapy treatment sessions ${ }^{[5,6,7]}$.

\section{(b) Statistical analyses}

The database and statistical analysis was performed using SPSS v.17 software. Measures such as mean, standard deviation, minimum and maximum range were developed from the continuous data. Relative frequency was calculated for discrete data. A p-value $<0.05$ was considered as statistical significant.

\section{Socio-demographic characteristics of the patients}

Table 1. Socio-demographic characteristics in the form of frequency and percentage of variables of the patients and correlation in the two groups, i.e., chemotherapy and radiotherapy

\begin{tabular}{|c|c|c|c|c|c|c|}
\hline \multirow{2}{*}{$\begin{array}{l}\text { S.No } \\
\text {. }\end{array}$} & \multirow[t]{2}{*}{ Variables } & \multirow[t]{2}{*}{ Parameters } & \multicolumn{2}{|c|}{ Chemotherapy } & \multicolumn{2}{|c|}{ Radiotherapy } \\
\hline & & & $\begin{array}{l}\text { Frequency } \\
\text { (n) }\end{array}$ & $\begin{array}{l}\text { Percentage } \\
\text { (\%) }\end{array}$ & $\begin{array}{l}\text { Frequency } \\
\text { (n) }\end{array}$ & $\begin{array}{l}\text { Percentage } \\
\text { (\%) }\end{array}$ \\
\hline 1. & Gender & $\begin{array}{l}\text { a. Male } \\
\text { b. Female }\end{array}$ & $\begin{array}{l}6 \\
24\end{array}$ & $\begin{array}{l}20.0 \% \\
80.0 \%\end{array}$ & $\begin{array}{l}15 \\
15\end{array}$ & $\begin{array}{l}50.0 \% \\
50.0 \%\end{array}$ \\
\hline 2. & Age & $\begin{array}{l}\text { a. } 18-30 \text { years } \\
\text { b. } 30 \\
\text { c. } 46 \\
\text { d. } 60\end{array}$ & $\begin{array}{l}5 \\
17 \\
8\end{array}$ & $\begin{array}{l}16.7 \% \\
56.7 \% \\
26.7 \%\end{array}$ & $\begin{array}{l}6 \\
15 \\
9\end{array}$ & $\begin{array}{l}20.0 \% \\
50.0 \% \\
30.0 \%\end{array}$ \\
\hline 3. & $\begin{array}{l}\text { Marital } \\
\text { status }\end{array}$ & $\begin{array}{l}\text { a. Unmarried } \\
\text { b. Married } \\
\text { c. Widow } \\
\text { d. Divorced/ } \\
\quad \text { Legally } \\
\quad \text { separated } \\
\text { e. Others }\end{array}$ & 30 & $100.0 \%$ & $\begin{array}{l}1 \\
28 \\
1\end{array}$ & $\begin{array}{l}3.3 \% \\
93.3 \% \\
3.3 \%\end{array}$ \\
\hline 4. & $\begin{array}{l}\text { Educational } \\
\text { status }\end{array}$ & $\begin{array}{l}\text { a. Illiterate } \\
\text { b. Literate }\end{array}$ & 1 & $3.3 \%$ & 2 & $6.7 \%$ \\
\hline
\end{tabular}




\begin{tabular}{|c|c|c|c|c|c|c|}
\hline & & $\begin{array}{l}\text { i. Primary } \\
\text { ii. Secondary } \\
\text { iii.Tertiary }\end{array}$ & $\begin{array}{l}4 \\
10 \\
15\end{array}$ & $\begin{array}{l}13.3 \% \\
33.3 \% \\
50.0 \%\end{array}$ & $\begin{array}{l}5 \\
11 \\
12\end{array}$ & $\begin{array}{l}16.7 \% \\
36.7 \% \\
40.0 \%\end{array}$ \\
\hline 5. & Occupation & $\begin{array}{l}\text { a. Service } \\
\text { b. Business } \\
\text { c. Housewife } \\
\text { d. Freelancers } \\
\text { e. Pensioners } \\
\text { f. Domestic } \\
\text { duties } \\
\text { g. Cultivation }\end{array}$ & $\begin{array}{l}3 \\
3 \\
21 \\
3\end{array}$ & $\begin{array}{l}10.0 \% \\
10.0 \% \\
70.0 \% \\
10.0 \%\end{array}$ & $\begin{array}{l}10 \\
2 \\
11 \\
1 \\
4 \\
1 \\
1 \\
1\end{array}$ & $\begin{array}{l}33.3 \% \\
6.7 \% \\
36.7 \% \\
3.3 \% \\
13.3 \% \\
3.3 \% \\
\\
3.3 \% \\
\end{array}$ \\
\hline 6. & $\begin{array}{l}\text { Type of } \\
\text { family }\end{array}$ & $\begin{array}{l}\text { a. Nuclear } \\
\text { b. Joint }\end{array}$ & $\begin{array}{l}16 \\
14\end{array}$ & $\begin{array}{l}53.3 \% \\
46.7 \%\end{array}$ & $\begin{array}{l}9 \\
21\end{array}$ & $\begin{array}{l}30.0 \% \\
70.0 \%\end{array}$ \\
\hline 7. & Cohabitants & $\begin{array}{l}\text { a. Living } \\
\text { alone } \\
\text { b. Living with } \\
\text { partner } \\
\text { c. Living with } \\
\text { partner and } \\
\text { children } \\
\text { d. Living with } \\
\text { children }\end{array}$ & $\begin{array}{l}15 \\
1\end{array}$ & $\begin{array}{l}50.0 \% \\
3.3 \% \\
46.7 \%\end{array}$ & $\begin{array}{l}4 \\
5 \\
21\end{array}$ & $\begin{array}{l}13.3 \% \\
16.67 \% \\
70.0 \%\end{array}$ \\
\hline 8. & $\begin{array}{l}\text { Annual } \\
\text { income }\end{array}$ & $\begin{array}{l}\text { a. NA } \\
\text { b. } \leq 20 \\
\text { c. } 20 \\
\text { d. } 30 \\
\text { e. } 41 \\
\text { f. } \geq 84\end{array}$ & 22 & $73.3 \%$ & 11 & $36.7 \%$ \\
\hline 9. & $\begin{array}{l}\text { Place of } \\
\text { residence }\end{array}$ & $\begin{array}{l}\text { a. Small town } \\
\text { b. Big town }\end{array}$ & $\begin{array}{l}1 \\
29\end{array}$ & $\begin{array}{l}3.3 \% \\
96.7 \%\end{array}$ & $\begin{array}{l}8 \\
22\end{array}$ & $\begin{array}{l}26.7 \% \\
73.3 \%\end{array}$ \\
\hline
\end{tabular}

The mean age (SD) of the patients in Chemotherapy group: 54.37 (11.08) [Range: 32-75].

The mean age (SD) of the patients in Radiotherapy group: 54.21 (11.07) [Range: 32-75].

\section{Clinical characteristics of the patients}

Table 2: Clinicalcharacteristics in the form of frequency and percentage of variables of the patients and correlation in the two groups, i.e., chemotherapy or radiotherapy

\begin{tabular}{|l|l|l|l|l|l|l|}
\hline S.No. & Variables & Parameters & \multicolumn{2}{|l|}{ Chemotherapy } & \multicolumn{2}{l|}{ Radiotherapy } \\
\cline { 4 - 7 } & & $\begin{array}{l}\text { Frequency } \\
\text { (n) }\end{array}$ & $\begin{array}{l}\text { Percentage } \\
\text { (\%) }\end{array}$ & $\begin{array}{l}\text { Frequency } \\
\text { (n) }\end{array}$ & $\begin{array}{l}\text { Percentage } \\
\text { (\%) }\end{array}$ \\
\hline 1. & $\begin{array}{l}\text { Smoking } \\
\text { habit }\end{array}$ & a. Non-smoker & 27 & $90.0 \%$ & 27 & $90.0 \%$ \\
b. Ex-smoker & 3 & $10.0 \%$ & 4 & $10.0 \%$ \\
\hline 2. & $\begin{array}{l}\text { Drinking } \\
\text { habit }\end{array}$ & a. Non-drinker & 30 & $100.0 \%$ & 26 & $86.7 \%$
\end{tabular}


Texila International Journal of Clinical Research

Volume 3, Issue 2, Dec 2016

\begin{tabular}{|c|c|c|c|c|c|c|}
\hline & & b. Ex-drinker & & & 4 & $13.3 \%$ \\
\hline 3. & $\begin{array}{l}\text { Tobacco } \\
\text { use }\end{array}$ & $\begin{array}{l}\text { a. Yes } \\
\text { b. No } \\
\end{array}$ & $\begin{array}{l}1 \\
29 \\
\end{array}$ & $\begin{array}{l}3.3 \% \\
96.7 \% \\
\end{array}$ & $\begin{array}{l}7 \\
23 \\
\end{array}$ & $\begin{array}{l}23.3 \% \\
76.7 \% \\
\end{array}$ \\
\hline 4. & $\begin{array}{l}\text { Health } \\
\text { insurance }\end{array}$ & $\begin{array}{l}\text { a. Yes } \\
\text { b. No }\end{array}$ & $\begin{array}{l}23 \\
7 \\
\end{array}$ & $\begin{array}{l}76.7 \% \\
23.3 \% \\
\end{array}$ & $\begin{array}{l}19 \\
11 \\
\end{array}$ & $\begin{array}{l}63.3 \% \\
36.7 \% \\
\end{array}$ \\
\hline 5. & $\begin{array}{l}\text { Type of } \\
\text { health } \\
\text { insurance }\end{array}$ & $\begin{array}{l}\text { a. Government } \\
\text { medically } \\
\text { insured } \\
\text { b. TPA } \\
\text { c. Cash }\end{array}$ & $\begin{array}{l}10 \\
13 \\
7\end{array}$ & $\begin{array}{l}33.3 \% \\
43.3 \% \\
23.3 \%\end{array}$ & $\begin{array}{l}12 \\
7 \\
11\end{array}$ & $\begin{array}{l}40.0 \% \\
23.3 \% \\
36.7 \%\end{array}$ \\
\hline 6. & $\begin{array}{l}\text { Support by } \\
\text { charity } \\
\text { organizatio } \\
n\end{array}$ & $\begin{array}{l}\text { a. Yes } \\
\text { b. No }\end{array}$ & 30 & $100.0 \%$ & 30 & $100 \%$ \\
\hline 7. & $\begin{array}{l}\text { Cancer } \\
\text { tumor } \\
\text { location }\end{array}$ & $\begin{array}{l}\text { a. Ca Breast } \\
\text { b. Ca Head } \\
\text { c. Ca Neck }\end{array}$ & $\begin{array}{l}23 \\
1 \\
6\end{array}$ & $\begin{array}{l}76.7 \% \\
3.3 \% \\
20.0 \%\end{array}$ & $\begin{array}{l}10 \\
5 \\
15\end{array}$ & $\begin{array}{l}33.3 \% \\
16.7 \% \\
50.0 \%\end{array}$ \\
\hline 8. & $\begin{array}{l}\text { Disease } \\
\text { acceptance }\end{array}$ & $\begin{array}{l}\text { a. Yes } \\
\text { b. No }\end{array}$ & $\begin{array}{l}23 \\
7\end{array}$ & $\begin{array}{l}76.7 \% \\
23.3 \% \\
\end{array}$ & $\begin{array}{l}15 \\
15\end{array}$ & $\begin{array}{l}50.0 \% \\
50.0 \% \\
\end{array}$ \\
\hline 9. & $\begin{array}{l}\text { Reproductiv } \\
\text { e age of } \\
\text { women }\end{array}$ & $\begin{array}{l}\text { a. Pre- } \\
\text { menopausal } \\
\text { b. Menopausal }\end{array}$ & $\begin{array}{l}10 \\
14 \\
\end{array}$ & $\begin{array}{l}33.3 \% \\
46.7 \% \\
\end{array}$ & 9 & $\begin{array}{l}30.0 \% \\
20.0 \% \\
\end{array}$ \\
\hline 10. & Cancer type & $\begin{array}{l}\text { a. Primary } \\
\text { cancer } \\
\text { b. Recurrent } \\
\text { cancer } \\
\end{array}$ & 30 & $100.0 \%$ & 30 & $100.0 \%$ \\
\hline 11. & $\begin{array}{l}\text { Co- } \\
\text { existence of } \\
\text { metastasis }\end{array}$ & $\begin{array}{l}\text { a. Yes } \\
\text { b. No }\end{array}$ & $\begin{array}{l}8 \\
22\end{array}$ & $\begin{array}{l}26.7 \% \\
73.4 \%\end{array}$ & 30 & $100.0 \%$ \\
\hline 12. & $\begin{array}{l}\text { Chemothera } \\
\text { py's cycle } \\
\text { during the } \\
\text { interview of } \\
\text { QLQ }\end{array}$ & $\begin{array}{l}\text { b. } 2^{\text {nd }} \\
\text { c. } 3^{\text {rd }}\end{array}$ & $\begin{array}{l}2 \\
15\end{array}$ & $\begin{array}{l}6.7 \% \\
50.0 \%\end{array}$ & & \\
\hline
\end{tabular}




\begin{tabular}{|c|l|l|l|l|l|l|} 
& & $\begin{array}{l}\text { d. } 4^{\text {th }} \\
\text { e. } 5^{\text {th }} \\
\text { f. } 6^{\text {th }}\end{array}$ & $\begin{array}{l}6 \\
6\end{array}$ & $\begin{array}{l}20.0 \% \\
20.0 \% \\
3.3 \%\end{array}$ & \\
\hline 13 & $\begin{array}{l}\text { Radiotherap } \\
\text { y's cycle } \\
\text { during the } \\
\text { interview of } \\
\text { QLQ }\end{array}$ & a. $10^{\text {th }}-15^{\text {th }}$ & & & 5 & $16.7 \%$ \\
& $\begin{array}{l}\text { b. } 16^{\text {th }}-20^{\text {th }} \\
\text { c. } 21^{\text {st }}-25^{\text {th }} \\
\text { d. } 26^{\text {th }} 30^{\text {th }}\end{array}$ & & & & \\
& & & 21 & $70.0 \%$ \\
& & 1 & $10.0 \%$ \\
\end{tabular}

QOL domain scores for chemotherapy sessions $(n=30)$

Table 3. QOL domain scores for chemotherapy sessions $(\mathrm{n}=30)$

\begin{tabular}{|l|l|l|l|l|l|}
\hline $\begin{array}{l}\text { Domains for } \\
\text { QOL/Scale }\end{array}$ & $\begin{array}{l}\text { Minimum } \\
\text { possible } \\
\text { raw score }\end{array}$ & $\begin{array}{l}\text { Maximum } \\
\text { possible raw } \\
\text { score }\end{array}$ & $\begin{array}{l}\text { Mean of } \\
\text { raw score }\end{array}$ & $\begin{array}{l}\text { SD of raw } \\
\text { score }\end{array}$ & $\begin{array}{l}\text { Mean of Score } \\
\text { translated on } \\
\text { a scale of 100 }\end{array}$ \\
\hline Physical & 7 & 35 & 17.97 & 3.09 & 39.66 \\
\hline Psychological & 6 & 30 & 17.94 & 1.87 & 49.76 \\
\hline Social & 3 & 15 & 7.77 & 0.89 & 41.66 \\
\hline Environmental & 8 & 40 & 24.83 & 1.94 & 55.00 \\
\hline ZSAS Scores & 20 & 80 & 53.30 & 9.10 & 66.62 \\
\hline ZSDS Scores & 20 & 80 & 46.94 & 5.63 & 58.66 \\
\hline
\end{tabular}

In the chemotherapy group, the physical domain was the most affected domain, followed by social domain, psychological and environmental domain.

QOL domain scores for Radiotherapy sessions $(n=30)$

Table 4. QOL domain scores for radiotherapy sessions $(n=30)$

\begin{tabular}{|l|l|l|l|l|l|}
\hline $\begin{array}{l}\text { Domains for } \\
\text { QOL/Scale }\end{array}$ & $\begin{array}{l}\text { Minimum } \\
\text { possible raw } \\
\text { score }\end{array}$ & $\begin{array}{l}\text { Maximum } \\
\text { possible raw } \\
\text { score }\end{array}$ & $\begin{array}{l}\text { Mean of } \\
\text { raw score }\end{array}$ & $\begin{array}{l}\text { SD of } \\
\text { raw score }\end{array}$ & $\begin{array}{l}\text { Mean of } \\
\text { Score } \\
\text { translated on } \\
\text { a scale of 100 }\end{array}$ \\
\hline Physical & 7 & 35 & 17.37 & 4.18 & 37.13 \\
\hline Psychological & 6 & 30 & 17.74 & 2.78 & 49.10 \\
\hline Social & 3 & 15 & 7.77 & 1.25 & 40.73 \\
\hline Environmental & 8 & 40 & 25.10 & 2.35 & 55.26 \\
\hline ZSAS Scores & 20 & 80 & 43.17 & 8.04 & 53.95 \\
\hline ZSDS Scores & 20 & 80 & 41.70 & 6.05 & 52.12 \\
\hline
\end{tabular}

In the radiotherapy group, the physical domain was the most affected domain, followed by social domain, psychological and environmental domain.

Measuring ability of a cancer patient to consent in chemotherapy as well as, radiotherapy group while participating in QOL assessment 
Texila International Journal of Clinical Research

Volume 3, Issue 2, Dec 2016

Table 5. Measuring ability of a cancer patient to consent in chemotherapy as well as, radiotherapy group.

\begin{tabular}{|l|l|l|l|l|}
\hline \multirow{2}{*}{$\begin{array}{l}\text { Process of } \\
\text { Questionnaire } \\
\text { completion }\end{array}$} & $\begin{array}{l}\text { CHEMOTHERAPY } \\
\text { (n= 30) }\end{array}$ & $\begin{array}{l}\text { RADIOTHERAPY } \\
\text { (n= 30) }\end{array}$ \\
\cline { 2 - 5 } & $\mathbf{n}$ & $\begin{array}{l}\text { Percentag } \\
\text { e (\%) }\end{array}$ & $\mathbf{n}$ & $\begin{array}{l}\text { Percentag } \\
\text { e (\%) }\end{array}$ \\
\hline Patient themselves & 20 & $66.6 \%$ & 23 & $76.7 \%$ \\
\hline Assisted by relatives & 2 & $6.7 \%$ & 3 & $10.0 \%$ \\
\hline Assisted by researcher & 8 & $26.7 \%$ & 4 & $13.3 \%$ \\
\hline
\end{tabular}

It can be observed from table no. 3 and 4that in the chemotherapy group whose anxiety level (Mean= 66.62) and depression level (Mean= 58.66) as measured by ZSAS and ZSDS, respectively, patients were able to consent themselves 20(66.6\%), assisted by relatives/caregivers 2(6.7\%), and assisted by the researcher 8 (26.7\%) in order to, particpate in the research study entitled to "QOL of cancer patients during inpatient chemotherapy or outpatient radiotherapy".

On the other hand, in the radiotherapy group whose anxiety level (Mean= 53.95) and depression level (Mean= 52.12) as measured by ZSAS and ZSDS, respectively, patients were able to consent themselves 23(76.7\%), assisted by relatives/caregivers 3(10.0\%), and assisted by the researcher $4(13.3 \%)$ in order to, particpate in the research study entitled to "QOL of cancer patients during inpatient chemotherapy or outpatient radiotherapy”.

\section{Discussion}

The present study aimed at how the cancer patient undergoing either chemotherapy or radiotherapy sessions consented their ability to participate in the study entitled to "QOL of cancer patients during inpatient chemotherapy or outpatient radiotherapy treatment”. From the data retrieved it was observed that though cancer patients were suffering from the phase of anxiety and depression during the course of their treatment, yet maximum of the patients in both the treatment groups were able to consent their participation in the present research survey. All the studies published so far have included the standardized tools for measuring Medical competence to consent. However, in the present study, there was no such tool was utilized instead a general part is presented while assessing for the QOL of cancer patients during the chemotherapy or radiotherapy sessions.

\section{Conclusion}

Therefore, it could be concluded that Medical capacity to consent for participating in research study is an important aspect in cancer population as well. During the chemotherapy sessions or radiotherapy sessions, the cognitive ability gets affected. So, while conducting research survey it is important that the care-giver along with the researcher should be present with the cancer patient (subject) at the time of interview (research study).

\section{Future directions}

a. Larger sample size for the evaluation of the research study could be used.

b. Standardized assessment tools for medical capacity to consent in cancer patients could be used in order to, understand and analyze QOL of cancer patients during chemotherapy or radiotherapy treatment. 


\section{Limitations of the study}

a. Small sample size, i.e., Chemotherapy group $(n=30)$ and Radiotherapy group $(n=30)$ were included in the study.

b. Both the genders should have been equally included in the study so as to obtain gender specific significant differences.

\section{Footnotes}

Author's Contribution: 1- acquisition of data; 2- analysis and interpretation of data; 3- drafting of the manuscript; 4- critical revision of the manuscript for important intellectual content; 5 - statistical analysis; 6- administrative, technical and material supports.

Funding/Support: Self-funded.

\section{Acknowledgement}

The present study is a part of the research programme, “Assessment of Quality of Life of Adult Cancer Patients during inpatient Chemotherapy Treatment (CT), or outpatient Radiotherapy Treatment (RT) in a tertiary care hospital of New Delhi, India”. This research was supportedand assisted by at:

Department of Medical Oncology and Radiation Oncology, DR. B.L. Kapur Memorial Hospital, New Delhi, India.

\section{Under the Supervision of}

Dr. S. Hukku (H.O.D. of Radiation Oncology Department),

DR. B.L. Kapur Memorial Hospital,

New Delhi, India.

Dr. Amit Agarwal (H.O.D. of Medical Oncology Department),

DR. B.L. Kapur Memorial Hospital,

New Delhi, India.

\section{Under the Guidance of:}

Dr. Renita Bhamrah (Texila American University),

E-mail:darpreetrenita@gmail.com

\section{References}

[1]. Ananthanarayanan, R. M., Janardhan, V., Kannan, G., Nigam, N., Palani, T., Rani, V., et al. (2011). Assessment of quality of life of cancer patients in a tertiary care hospital of South India. Journal of Cancer Research and Therapeutics, 275-279. Available at:http://medind.nic.in/jat/t11/i3/jatt11i3p275.htm

[2]. Nichita, E. C., \& Buckley, P. F. (2007). Informed Consent and Competency: Doctor's Dilemma on the Consultation Liaison Service. Psychiatry (Edgmont), 4(3), 53-55.

https://www.ncbi.nlm.nih.gov/pmc/articles/PMC2922359/

[3]. Sturman, E. D. (2005). The capacity to consent to treatment and research: A review of standardized assessment tools. Clinical Psychology Review, 954 - 974.

http://users.phhp.ufl.edu/rbauer/forensic_neuropsychology/capacity_consent_treat_research_05.pdf

[4]. Triebel, K. L., Martin, R. C., Nabors, L. B., \& Marson, D. C. (2009). Medical decision-making capacity in patients with malignant glioma. Neurology, 73(24), 2086-2092.

http://doi.org/10.1212/WNL.0b013e3181c67bce

[5]. WHOQOL Group. (1998). Development of the World Health Organization WHOQOLBREF quality of life assessment. Psychol Med, 28:551-8.

[6]. Zung, W. W. K. (1971). A rating instrument for anxiety disorders. Psychosomatics, 12(6), 371-379. Available at: https://psychology-tools.com/zung-anxiety-scale/

[7]. Zung, W. W. K. (1965). A Self-Rating Depression Scale. (1965). Arch Gen Psychiatry, 12: 63-70. Available at: https://psychology-tools.com/zung-depression-scale/ 


\title{
Anxiety \& Depression Assessment of cancer patients undergoing Chemotherapy or Radiotherapy Treatment by the use of computerized developed version of Questionnaire (WHOQOL-Bref, ZSAS, ZSDS)
}

\author{
Article by Kavita Gupta \\ Ph.D. Clinical Research, Texila American University, India \\ E-mail: 16kavitagupta@texilaconnect.com
}

\begin{abstract}
Background: The quality of life of cancer patients during chemotherapy sessions or radiotherapy sessions encompasses several changes. However, patient had to suffer a lot during the sessions. In order to, measure their quality of life through manual way, it is advisable to implement the computerized electronic version of QLQ so that the patient could deal with the different questions independently without hesitation that might led to increase in cognitive ability during the sessions. Moreover, the healthcare professionals could direct and plan the treatment accordingly so as to improve the QOL of cancer patients.

Objectives: The overarching purpose of this study had been to evaluate the acceptability of QOL assessments among healthcare professionals and cancer patients, by addressing how QOL assessments could be utilized to predict anxiety and depression scores, and by revealing how technology could contribute to the accuracy of QOL assessments and facilitate their implementation into oncology practices. The introduction of the electronic version of the QLQ could however, benefit the overall QOL of patients by bridging the gap between research and clinical practice among Indian population.

Methods: It was a cross-sectional, descriptive, hospital based evaluation study. Total duration of the study was 5 months (December 2015- April 2016), conducted in Medical and Radiation Oncology department of Dr. B.L. Kapur Memorial Hospital, New Delhi, India. A convenience sample of 60 patients with cancer was selected. They were further divided into Chemotherapy group $(n=30)$ and Radiotherapy group $(n=30)$. Data was obtained through direct interview, using validated Psychological intervention tool in the form of Questionnaire: WHOQOL-Bref Questionnaire, Zung Self-Rating Anxiety scale and Zung Self-Rating Depression scale, which was further managed through a statistical program, using appropriate statistical tests.

Results: A total of 60 cancer patients were included in the study in which Chemotherapy group consisted of 30n cancer patients and Radiotherapy group consisted of 30n cancer patients. In the study, 6(20\%) were males and 24(80\%) were females in the chemotherapy group, and, 15(50\%) were males and $15(50 \%)$ were females in the radiotherapy group. Majority of the patients 32(53.34\%) were in the age range of 46-60 years.

Conclusion: Utilizing technology to implement QOL assessments into clinical practice has several advantages that make it more feasible for physicians to use QOL information with their patients for predicting and determining anxiety and depression scores during cancer treatment. Electronic methods of assessment are more accurate and less time-consuming than paper-and pencil questionnaires (manual method).
\end{abstract}

Keywords: Cancer, Chemotherapy, Radiotherapy, Quality of Life, WHOQOL-Bref Questionnaire, Zung Self-Rating Anxiety scale, Zung Self-Rating Depression scale, Computerized QOL, Electronic version. 
Texila International Journal of Clinical Research

Volume 3, Issue 2, Dec 2016

$\begin{array}{ll}\text { Abbreviations } & \\ \text { QOL } & : \text { Quality of life } \\ \text { QLQ } & : \text { Quality life Questionnaire } \\ \text { WHOQOL-Bref } & : \text { World Health Organization Quality of life assessment-a short brief version } \\ \text { ZSAS } & : \text { Zung Self-Rating Anxiety scale } \\ \text { ZSDS } & : \text { Zung Self-Rating Depression scale } \\ \text { CT } & : \text { Chemotherapy Treatment } \\ \text { RT } & : \text { Radiotherapy Treatment } \\ \text { DOM } & : \text { Third Party Administrator } \\ \text { TPA } & : \text { Fine needle aspiration cytology } \\ \text { FNAC } & \text { Standard deviation. }\end{array}$

\section{Introduction}

QOL assessmentis an important aspect in cancer patients because they provide insights into life domains affected either by the symptoms of cancer-related or cancer-treatment related or both ${ }^{[1]}$. This knowledge is significant because many cancer patients stressed that quality of life was just as important, if not more important, than quantity of life ${ }^{[1]}$. QLQ serves as a communication evaluation tool in which assessment of records usually reflect the level of care given to a cancer patient by the physician and healthcare professionals ${ }^{[1]}$. In order to provide timely response to patients' needs, QOL should be embedded into the care process of patient reported outcomes by redesigning care, particularly by paying attention to principles of effective distribution as well as implementing new infrastructures and technologies ${ }^{[1]}$. Electronic version of QLQ record of cancer patients is a real-time, point-of-care, patient-centric information resource for clinicians that represent a major domain of health information technology (HIT) ${ }^{[1]}$. In order to have the ideal computerized assessment system, it must be clinically relevant (useful and presented in a timely manner), sensitive to change, culturally sensitive, low burden, low in cost, built into the standard operating procedures, and meet regulatory, consumer, and community requirements ${ }^{[1]}$. The effort that physicians take to inquire about QOL improves doctor-patient communication and shows patients that their physicians care about them and are interested in their well-being ${ }^{[1]}$. To patients, this is highly meaningful ${ }^{[1]}$.

\section{Aims}

The main objectives of the present study was:

i. To determine and evaluate the time duration in assessment of anxiety and depression scores for the assessment of quality of life in cancer patients by the use of WHOQOL-Bref, Zung Selfrating Anxiety Scale (ZSAS) and Zung Self Rating Depression Scale (ZSDS) questionnaire manually during chemotherapy treatment or radiotherapy treatment.

ii. To develop innovative electronic version (computerized assessment) of WHOQOL-Bref, ZSAS, and ZSDS Questionnaireto be implemented in the hospital in order to, evaluate quality of life in the cancer patients during chemotherapy treatment or radiotherapy treatment. 


\section{Patients and methods}

The proposed study was conducted in accordance and adherence to the Ethical Guidelines and Procedures. Special care of the potential risks due to emotional distress was taken care of so that the dignity of the subject was not harmed. The authorized Ethical approval from the IRB and Ethical committee of Dr. B.L. Kapur Memorial Hospital, New Delhi, with Ref. No.: IRB/AARCE/5/DEC/2015/1, dated December $7^{\text {th }}$, 2015, was obtained to carry out the research study. Thereafter, the patients and their caregivers were approached in the inpatient as well as, outpatient clinic, where the purpose of the study was explained and they were invited to participate. Patients who agreed to participate were asked to sign an Informed Consent Form followed by the implementation of the Structured and Validated instrumental tool of WHOQOL-Bref, Zung Self-Rating Anxiety Scale (ZSAS), and Zung Self-Rating Depression Scale (ZSDS) in the form of questionnaire which lasted for approximately 25-60 minutes. The RESEARCH DESIGN of the proposed approved study protocol included 30n cancer patients undergoing Chemotherapy Treatment and 30n cancer patients undergoing Radiotherapy Treatment session. The inclusion criteria for the approved study were the patients with Breast cancer, sub-sites of head and neck tumors (e.g., nasopharyngeal, thyroid cancer, and parotid tumors), aged 18 years or older, Clinically diagnosed and confirmed by biopsy or FNAC, Undergoing/during the treatment sessions ( $\geq 2$ cycles and $\leq 6$ for Chemotherapy treatment, and $\geq 10$ cycles and $\leq 30$ cycles for Radiotherapy treatment, Voluntarily agreed to join the study, and aware of diagnosis and predicted prognosis. However, the study excluded patients with Inadequate clinical condition (ambulatory and terminally ill patients) who were unable to respond to an interview, had difficulty in understanding the questionnaire or communicating, were serious and didn't give consent, had a history of psychiatric disorder. Moreover, the study did not include the dosage of chemotherapy drugs and irradiation treatment. Eligible patients were identified through an institutional database or by referring physicians and were approached at their simulation appointment.

\section{Results}

\section{(a) Data collection}

Treatment-related symptoms were assessed by using a series of interviews through standard questionnaires of WHOQOL-Bref, the core questionnaire, followed by Zung Self-Rating Anxiety Scale (ZSAS) and Zung Self Rating Depression Scale (ZSDS) Questionnaires. These questionnaires have been proven to have good validity and reliability properties, cross-culturally accepted instrument to measure Quality of Life in cancer patients and are publicly available for scholar research purposes $[2,3,4]$. The questionnaire was provided in a language that the patient could understand easily (English / Hindi) followed by face-to-face interview of the patient who was either undergoing chemotherapy or radiotherapy treatment sessions.

Parts of the Record Card:

i. Personal information form:This first part contained patient information. The form was prepared, based on the literature. It contained age, gender, qualification, marital status, family type, Occupation, data on financial income and site of tumor location ${ }^{[2]}$.

ii. WHOQOL-Bref Questionnaire: This is an abbreviated version of the instrument WHOQOL-100. It consisted of 26 questions, being two about quality of life in general and other 24 representing each of the facets that made up the original instrument ${ }^{[2]}$. The questions were organized in 4 domains:

a. Physical domain (DOM1): It included7 questions pertaining to sleep, energy, mobility, the extent to which pain prevents performance of necessary tasks, the need for medical treatment to function in daily life, level of satisfaction with their capacity for work ${ }^{[2]}$.

b. Psychological domain (DOM2): It included6 questions pertaining to the ability to concentrate, self-esteem, body image, spirituality i.e. the extent to which they feel their life is meaningful, the frequency of positive or negative feelings i.e. blue mood, despair, anxiety, depression ${ }^{[2]}$. 
Texila International Journal of Clinical Research

Volume 3, Issue 2, Dec 2016

c. Social domain (DOM3): It included 3 questions pertaining to satisfaction with personal relationships, social support systems and sexual satisfaction ${ }^{[2]}$.

d. Environmental domain (DOM4): It included 8 questions related to safety and security, home and physical environment satisfaction, finance i.e. does the respondent have enough money to meet their needs, health/social care availability, information and leisure activity accessibility and transportation satisfaction ${ }^{[2]}$.

Equations for computing domain raw scores:

Domain 1 (Physical) score = (6- Q3) + (6-Q4) + Q5 + Q6 + Q7 + Q8 + Q9

Domain 2 (Psychological) score = Q10 + Q11 + Q12 + Q13 + Q14 + (6-Q15)

Domain 3 (Social) score $=\mathrm{Q} 16+\mathrm{Q} 17+\mathrm{Q} 18$

Domain 4 (Environmental) score = Q19 + Q20 + Q21 + Q22 + Q23 + Q24 + Q25 + Q26 ${ }^{[2]}$.

In addition to the 4 domains, the WHOQOL-Bref included two stand-alone questions, one pertaining to the respondents' rated QOL, and one related to their Satisfaction with Health were analyzed separately ${ }^{[2]}$. The score of each question ranged from 1 to 5 on a 5 point likert scale and higher scores indicated a better evaluation. Raw scores of the respective domains were then transformed from 0-100 with the lowest score of zero and the highest score of 100according to the accepted guidelines.

iii. ZSAS: Zung Self-Rating Anxiety scale quality life questionnaire is a likert scale format (scoring on 1 to 4 scale) that was built by a psychiatrist, William W. K. Zung to measure the rate of anxiety. The scale consisted of 20 self-reported items with 15 questions of increasing anxiety level and 5 questions of decreasing anxiety level (Q. no. 5, 9, 13, 17, 19) ${ }^{[3]}$. Scores for each question ranged from 1 to 4 and higher scores indicated severe anxiety level. The raw scores were counted up and multiplied by 1.25 to reach a standardized score, according to the instructions that accompanied the scale ${ }^{[3]}$. The ZSAS Index score followed the crierion: Normal Range (20-44); Mild to Moderate Anxiety level (45-59); Marked to Severe Anxiety level (60-74); Extreme Anxiety level (75-80) ${ }^{[3]}$.

iv. ZSDS: Zung Self-Rating Depression scale quality life questionnaire is also a 20 items short self-administered survey that was designed by William W. K. Zung to assess the level of four common characteristics of depression for patients: the pervasive effect, the physiological equivalents, other disturbances, and psychomotor activities. There were ten positively worded and ten negatively worded questions. Each question was scored on a scale of 1-4 (a little of the time, some of the time, good part of the time, most of the time) ${ }^{[4]}$. The higher scores indicated severe depression level. The raw scores were counted up and multiplied by 1.25 to reach a standardized score, according to the instructions that accompanied the scale ${ }^{[4]}$. The ZSDS Index score followed the crierion: Normal Range $(<50)$; Mild Depression level $(<60)$; Moderate Depression level $(<70)$; Extreme Depression level $(>70){ }^{[4]}$.

\section{(b) Statistical analyses}

The database and statistical analysis was performed by using SPSS v.17 software. The independent variables analyzed were Socio-demographic characteristics (sex/gender, age, education level, marital status, employment type, income level (per annum), job background, local residence), Clinical characteristics (smoking habit, drinking habit, tobacco use, health insurance, tumor type \& location, metastasis involved, corresponding cycle number for both chemotherapy and radiotherapy treatment); Anxiety and Depression of the patients as assessed by ZSAS and ZSDS respectively. The dependent variables included: subscale and overall QOL scores, and Health satisfaction as measured by WHOQOL-Bref Questionnaire (Tool/instrument). Descriptive statistics computation techniques were applied to the discrete and continuous data. Measures such as mean, standard deviation, minimum and maximum range were developed from the continuous data. Relative frequency was calculated for discrete data. Mean with Standard deviation (SD) was used to summarize the age of patients. Chisquare test was performed to assess the effect of different sociodemographic factors and clinical characteristics on the QOL of the cancer patients. Student's $t$-test was used to compare sample means 
for study variables (anxiety, depression and QOL). Bivariate analysis was performed to assess the predictors of QOL. Based on the survey/ research, Pearson Correlation coefficient test denoted by $r$ was calculatedto assess the particular characteristic symptomatic function of anxiety and depression instrument that significantly affected the QOL domains, i.e., either positively or negatively. Paired ttest was used to compare difference between score means of different domains. A $p$-value $<0.05$ was considered as statistical significant.

\section{Socio-Demographic characteristics of the patients}

Table 1. Socio-demographic characteristics in the form of Frequency and percentage of variables of the patients and correlation in the two groups, i.e., Chemotherapy and Radiotherapy

\begin{tabular}{|c|c|c|c|c|c|c|}
\hline \multirow[t]{2}{*}{ S.No } & \multirow[t]{2}{*}{ Variables } & \multirow[t]{2}{*}{ Parameters } & \multicolumn{2}{|c|}{ Chemotherapy } & \multicolumn{2}{|c|}{ Radiotherapy } \\
\hline & & & $\begin{array}{l}\text { Frequency } \\
\text { (n) }\end{array}$ & $\begin{array}{l}\text { Percentage } \\
\text { (\%) }\end{array}$ & $\begin{array}{l}\text { Frequency } \\
\text { (n) }\end{array}$ & $\begin{array}{l}\text { Percentage } \\
\text { (\%) }\end{array}$ \\
\hline 1. & Gender & $\begin{array}{l}\text { a. Male } \\
\text { b. Female }\end{array}$ & $\begin{array}{l}6 \\
24\end{array}$ & $\begin{array}{l}20.0 \% \\
80.0 \%\end{array}$ & $\begin{array}{l}15 \\
15\end{array}$ & $\begin{array}{l}50.0 \% \\
50.0 \%\end{array}$ \\
\hline 2. & Age & $\begin{array}{l}\text { a. } 18-30 \text { years } \\
\text { b. } 30 \\
\text { c. } 46 \\
\text { d. } 60\end{array}$ & $\begin{array}{l}5 \\
17 \\
8\end{array}$ & $\begin{array}{l}16.7 \% \\
56.7 \% \\
26.7 \%\end{array}$ & $\begin{array}{l}6 \\
15 \\
9\end{array}$ & $\begin{array}{l}20.0 \% \\
50.0 \% \\
30.0 \%\end{array}$ \\
\hline 3. & $\begin{array}{l}\text { Marital } \\
\text { status }\end{array}$ & $\begin{array}{l}\text { a. Unmarried } \\
\text { b. Married } \\
\text { c. Widow } \\
\text { d. Divorced/ } \\
\quad \text { Legally } \\
\quad \text { separated } \\
\text { e. Others }\end{array}$ & 30 & $100.0 \%$ & $\begin{array}{l}1 \\
28 \\
1\end{array}$ & $\begin{array}{l}3.3 \% \\
93.3 \% \\
3.3 \%\end{array}$ \\
\hline 4. & $\begin{array}{l}\text { Educational } \\
\text { status }\end{array}$ & $\begin{array}{l}\text { a. Illiterate } \\
\text { b. Literate }\end{array}$ & 1 & $3.3 \%$ & 2 & $6.7 \%$ \\
\hline & & $\begin{array}{l}\text { i. Primary } \\
\text { ii. Secondary } \\
\text { iii.Tertiary }\end{array}$ & $\begin{array}{l}4 \\
10 \\
15\end{array}$ & $\begin{array}{l}13.3 \% \\
33.3 \% \\
50.0 \%\end{array}$ & $\begin{array}{l}5 \\
11 \\
12\end{array}$ & $\begin{array}{l}16.7 \% \\
36.7 \% \\
40.0 \%\end{array}$ \\
\hline 5. & Occupation & $\begin{array}{l}\text { a. Service } \\
\text { b. Business } \\
\text { c. Housewife } \\
\text { d. Freelancers } \\
\text { e. Pensioners } \\
\text { f. Domestic } \\
\quad \text { duties } \\
\text { g. Cultivation }\end{array}$ & $\begin{array}{l}3 \\
3 \\
21 \\
3\end{array}$ & $\begin{array}{l}10.0 \% \\
10.0 \% \\
70.0 \% \\
10.0 \%\end{array}$ & $\begin{array}{l}10 \\
2 \\
11 \\
1 \\
4 \\
1 \\
1\end{array}$ & $\begin{array}{l}33.3 \% \\
6.7 \% \\
36.7 \% \\
3.3 \% \\
13.3 \% \\
3.3 \% \\
3.3 \%\end{array}$ \\
\hline 6. & $\begin{array}{l}\text { Type of } \\
\text { family }\end{array}$ & $\begin{array}{l}\text { a. Nuclear } \\
\text { b. Joint }\end{array}$ & $\begin{array}{l}16 \\
14\end{array}$ & $\begin{array}{l}53.3 \% \\
46.7 \%\end{array}$ & $\begin{array}{l}9 \\
21\end{array}$ & $\begin{array}{l}30.0 \% \\
70.0 \%\end{array}$ \\
\hline 7. & Cohabitants & $\begin{array}{l}\text { a. Living } \\
\text { alone } \\
\text { b. Living with }\end{array}$ & 15 & $50.0 \%$ & 4 & $13.3 \%$ \\
\hline
\end{tabular}


Texila International Journal of Clinical Research

Volume 3, Issue 2, Dec 2016

\begin{tabular}{|l|l|l|l|l|l|l|} 
& & $\begin{array}{l}\text { partner } \\
\text { c. Living with } \\
\text { partner and } \\
\text { children } \\
\text { d. Living with } \\
\text { children }\end{array}$ & 14 & $3.3 \%$ & 5 & $16.67 \%$ \\
\hline 8. & $\begin{array}{l}\text { Annual } \\
\text { income }\end{array}$ & $\begin{array}{l}\text { a. NA } \\
\text { b. } \leq 20 \\
\text { c. } 20 \\
\text { d. } 30 \\
\text { e. } 41 \\
\text { f. } \geq 84\end{array}$ & 22 & $73.3 \%$ & 11 & $30.0 \%$ \\
\hline 9. & $\begin{array}{l}\text { Place of } \\
\text { residence }\end{array}$ & $\begin{array}{l}\text { a. Small town } \\
\text { b. Big town }\end{array}$ & 1 & 29 & $26.7 \%$ & 19 \\
\hline
\end{tabular}

(All tests were performed using Pearson $\chi 2$ test for association analysis)

\section{Clinical characteristics of the patients}

Table 2. Clinicalcharacteristics in the form of Frequency and percentage of variables of the patients and correlation in the two groups, i.e., Chemotherapy or Radiotherapy

\begin{tabular}{|c|c|c|c|c|c|c|}
\hline \multirow[t]{2}{*}{ S.No. } & \multirow[t]{2}{*}{ Variables } & \multirow[t]{2}{*}{ Parameters } & \multicolumn{2}{|c|}{ Chemotherapy } & \multicolumn{2}{|c|}{ Radiotherapy } \\
\hline & & & $\begin{array}{l}\text { Frequency } \\
\text { (n) }\end{array}$ & $\begin{array}{l}\text { Percentage } \\
\text { (\%) }\end{array}$ & $\begin{array}{l}\text { Frequency } \\
\text { (n) }\end{array}$ & $\begin{array}{l}\text { Percentage } \\
(\%)\end{array}$ \\
\hline 1. & $\begin{array}{l}\text { Smoking } \\
\text { habit }\end{array}$ & $\begin{array}{l}\text { a. Non-smoker } \\
\text { b. Ex-smoker }\end{array}$ & $\begin{array}{l}27 \\
3\end{array}$ & $\begin{array}{l}90.0 \% \\
10.0 \%\end{array}$ & $\begin{array}{l}27 \\
4\end{array}$ & $\begin{array}{l}90.0 \% \\
10.0 \%\end{array}$ \\
\hline 2. & $\begin{array}{l}\text { Drinking } \\
\text { habit }\end{array}$ & $\begin{array}{l}\text { a. Non-drinker } \\
\text { b. Ex-drinker }\end{array}$ & 30 & $100.0 \%$ & $\begin{array}{l}26 \\
4\end{array}$ & $\begin{array}{l}86.7 \% \\
13.3 \% \\
\end{array}$ \\
\hline 3. & $\begin{array}{l}\text { Tobacco } \\
\text { use }\end{array}$ & $\begin{array}{l}\text { a. Yes } \\
\text { b. No }\end{array}$ & $\begin{array}{l}1 \\
29\end{array}$ & $\begin{array}{l}3.3 \% \\
96.7 \%\end{array}$ & $\begin{array}{l}7 \\
23\end{array}$ & $\begin{array}{l}23.3 \% \\
76.7 \%\end{array}$ \\
\hline 4. & $\begin{array}{l}\text { Health } \\
\text { insurance }\end{array}$ & $\begin{array}{l}\text { a. Yes } \\
\text { b. No }\end{array}$ & $\begin{array}{l}23 \\
7 \\
\end{array}$ & $\begin{array}{l}76.7 \% \\
23.3 \% \\
\end{array}$ & $\begin{array}{l}19 \\
11 \\
\end{array}$ & $\begin{array}{l}63.3 \% \\
36.7 \% \\
\end{array}$ \\
\hline 5. & $\begin{array}{l}\text { Type of } \\
\text { health } \\
\text { insurance }\end{array}$ & $\begin{array}{l}\text { a. Government } \\
\text { medically } \\
\text { insured } \\
\text { b. TPA } \\
\text { c. Cash }\end{array}$ & $\begin{array}{l}10 \\
13 \\
7\end{array}$ & $\begin{array}{l}33.3 \% \\
43.3 \% \\
23.3 \%\end{array}$ & $\begin{array}{l}12 \\
7 \\
11\end{array}$ & $\begin{array}{l}40.0 \% \\
23.3 \% \\
36.7 \%\end{array}$ \\
\hline 6. & $\begin{array}{l}\text { Support by } \\
\text { charity } \\
\text { organizatio } \\
\mathrm{n}\end{array}$ & $\begin{array}{l}\text { a. Yes } \\
\text { b. No }\end{array}$ & 30 & $100.0 \%$ & 30 & $100 \%$ \\
\hline
\end{tabular}


Texila International Journal of Clinical Research

Volume 3, Issue 2, Dec 2016

\begin{tabular}{|c|c|c|c|c|c|c|}
\hline 7. & $\begin{array}{l}\text { Cancer } \\
\text { tumor } \\
\text { location }\end{array}$ & $\begin{array}{l}\text { a. Ca Breast } \\
\text { b. Ca Head } \\
\text { c. Ca Neck }\end{array}$ & $\begin{array}{l}1 \\
6\end{array}$ & $\begin{array}{l}76.7 \% \\
3.3 \% \\
20.0 \%\end{array}$ & $\begin{array}{l}5 \\
15\end{array}$ & $\begin{array}{l}33.3 \% \\
16.7 \% \\
50.0 \%\end{array}$ \\
\hline 8. & $\begin{array}{l}\text { Disease } \\
\text { acceptance }\end{array}$ & $\begin{array}{l}\text { a. Yes } \\
\text { b. No }\end{array}$ & $\begin{array}{l}23 \\
7\end{array}$ & $\begin{array}{l}76.7 \% \\
23.3 \% \\
\end{array}$ & $\begin{array}{l}15 \\
15\end{array}$ & $\begin{array}{l}50.0 \% \\
50.0 \% \\
\end{array}$ \\
\hline 9. & $\begin{array}{l}\text { Reproductiv } \\
\text { e age of } \\
\text { women }\end{array}$ & $\begin{array}{l}\text { a. Pre- } \\
\text { menopausal } \\
\text { b. Menopausal }\end{array}$ & $\begin{array}{l}10 \\
14\end{array}$ & $\begin{array}{l}33.3 \% \\
46.7 \%\end{array}$ & $\begin{array}{l}9 \\
6\end{array}$ & $\begin{array}{l}30.0 \% \\
20.0 \%\end{array}$ \\
\hline 10. & Cancer type & $\begin{array}{l}\text { a. Primary } \\
\text { cancer } \\
\text { b. Recurrent } \\
\text { cancer }\end{array}$ & 30 & $100.0 \%$ & 30 & $100.0 \%$ \\
\hline 11. & $\begin{array}{l}\text { Co- } \\
\text { existence of } \\
\text { metastasis }\end{array}$ & $\begin{array}{l}\text { a. Yes } \\
\text { b. No }\end{array}$ & $\begin{array}{l}8 \\
22\end{array}$ & $\begin{array}{l}26.7 \% \\
73.4 \%\end{array}$ & 30 & $100.0 \%$ \\
\hline 12. & $\begin{array}{l}\text { Chemothera } \\
\text { py’s cycle } \\
\text { during the } \\
\text { interview of } \\
\text { QLQ }\end{array}$ & $\begin{array}{l}\text { a. } 1^{\text {st }} \\
\text { b. } 2^{\text {nd }} \\
\text { c. } 3^{\text {rd }} \\
\text { d. } 4^{\text {th }} \\
\text { e. } 5^{\text {th }} \\
\text { f. } 6^{\text {th }}\end{array}$ & $\begin{array}{l}2 \\
15 \\
6 \\
6 \\
1\end{array}$ & $\begin{array}{l}6.7 \% \\
50.0 \% \\
20.0 \% \\
20.0 \% \\
3.3 \%\end{array}$ & & \\
\hline 13 & $\begin{array}{l}\text { Radiotherap } \\
\text { y's cycle } \\
\text { during the } \\
\text { interview of } \\
\text { QLQ }\end{array}$ & $\begin{array}{l}\text { a. } 10^{\text {th }}-15^{\text {th }} \\
\text { b. } 16^{\text {th }}-20^{\text {th }} \\
\text { c. } 21^{\text {st }}-25^{\text {th }} \\
\text { d. } 26^{\text {th }} 30^{\text {th }}\end{array}$ & & & $\begin{array}{l}21 \\
3 \\
1\end{array}$ & $\begin{array}{l}70.0 \% \\
10.0 \% \\
3.3 \%\end{array}$ \\
\hline
\end{tabular}


Time requirement in QOL Assessment process by the WHOQOL-BREF, ZSAS, ZSDS in both the chemotherapy group $(n=30)$ and radiotherapy group $(n=30)$, manually

Table 3: Time taken in QOL assessment through Questionnaires manually in both the groups, i.e., Chemotherapy $(n=30)$, and Radiotherapy $(n=30)$.

\begin{tabular}{|l|l|l|}
\hline $\begin{array}{l}\text { Process (Chemotherapy: } \mathbf{n} \\
\text { = 30; Radiotherapy: } \mathbf{n}=\end{array}$ & \multicolumn{2}{|l|}{ Time in minutes } \\
\cline { 2 - 3 } 30) & Range & (Mean \pm S.D.) \\
\hline $\begin{array}{l}\text { Researcher instructed how } \\
\text { to answer the questionnaire } \\
(\mathrm{n}=60)\end{array}$ & 29 & $(49.16 \pm 6.38)$ \\
\hline $\begin{array}{l}\text { Questionnaire completion } \\
(\mathrm{n}=60)\end{array}$ & 29 & $(49.16 \pm 6.38)$ \\
\hline $\begin{array}{l}\text { Patient themselves (n= 5) } \\
(30)\end{array}$ & 2 & $(57.00 \pm 1.00)$ \\
\hline $\begin{array}{l}\text { Assisted by relatives/care- } \\
\text { giver (n=7) }\end{array}$ & 4 & $(47.14 \pm 1.46)$ \\
\hline $\begin{array}{l}\text { Interviewed by researcher } \\
(\mathrm{n}=55)\end{array}$ & $(45-49)$ & \\
\hline
\end{tabular}

It was observed that the time taken by the patient to complete the questionnaires (WHOQOL-Bref, ZSAS, and ZSDS) either by himself or assisted by the researcher or by the patient's care-giver was minimum 30 minutes and maximum 59 minutes. This indicated that the total 66 questions for QOL assessments was a big tedious process.

\section{System specification for maximum and effective execution of the task by the system, there are the hardware and software requirement}

Hardware Requirement: System unit, Monitor (VDU), Uninterrupted power supply (UPS), 1 GB RAM, CD Rom, Hard disk capacity of 5 MB, Printer

Software Requirement: Window XP operating system (7/ 8// 10), Microsoft access, Microsoft visual basic, Dot Net framework 4.5.

Data items: Name of the patient, Age of the patient, Name of spouse, Name of the caregiver, Marital status of the patient, Gender, Address, Occupation, Educational status, Monthly income, Telephone number, Department of hospital, Hospital record number, Date of admission, Date of discharge, Hospital referred from, Hospital referred to, Primary physician, Other physicians, Diagnostic procedures, Tumor location, Mode of Treatment, Date of Admission and Date of Discharge. 


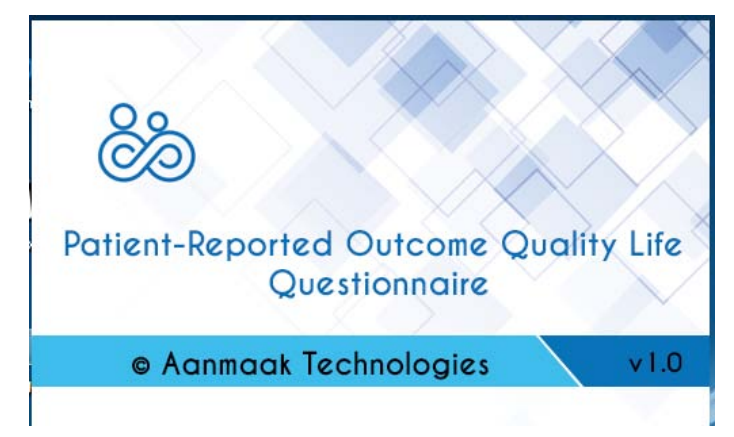

Fig. 1. Screenshot of the Software (Step 1)

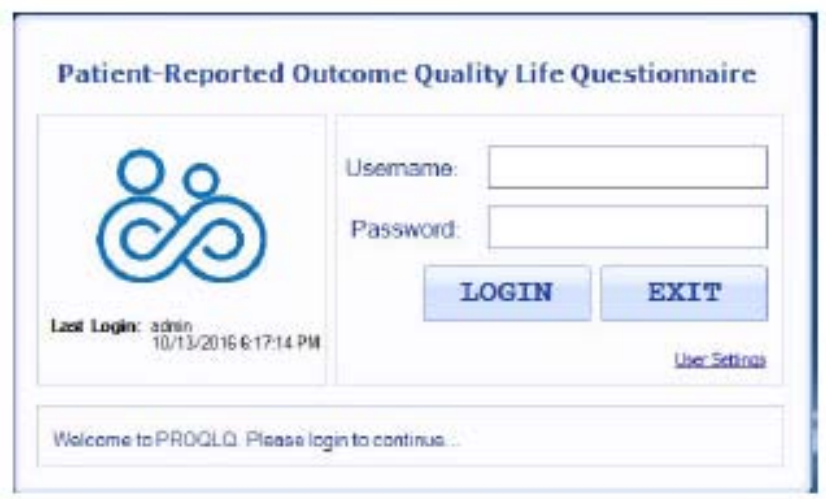

Fig. 2. Screenshot of the Software (Step 2)

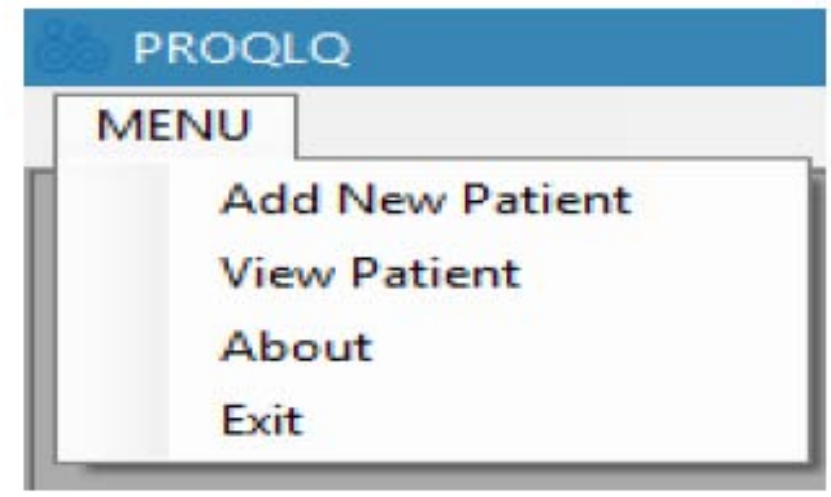

Fig. 3. Screenshot of the Software (Step 3)

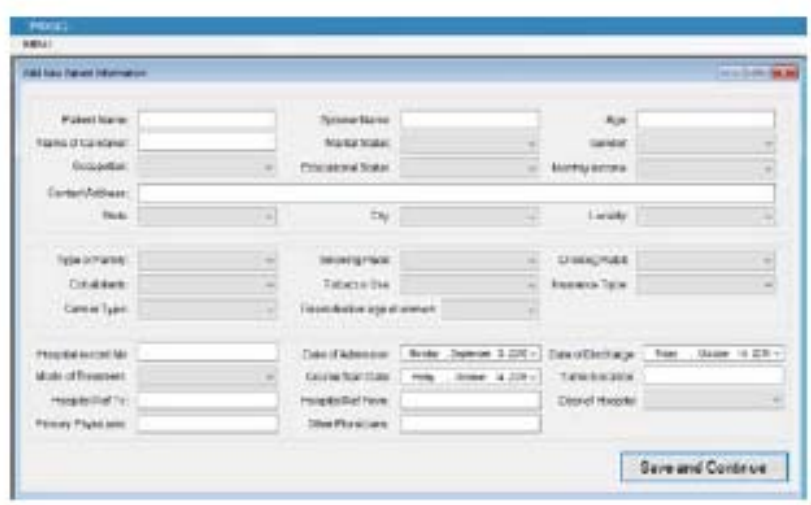


Texila International Journal of Clinical Research

Volume 3, Issue 2, Dec 2016

Fig. 4. Screenshot of the data items (Step 4)

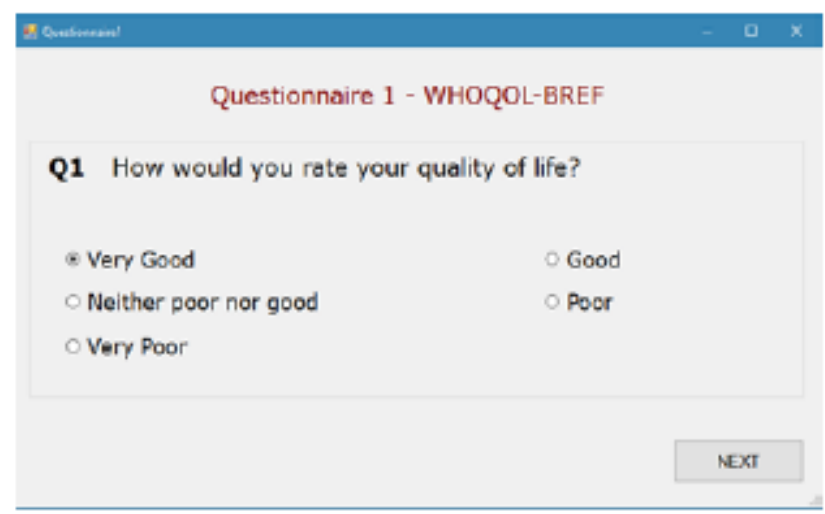

Fig. 5. Screenshot of the QLQ (Step 5)

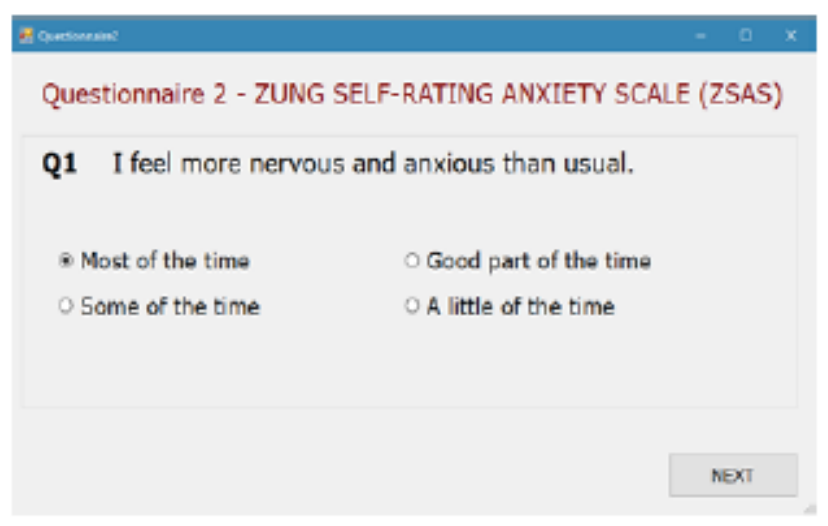

Fig. 6. Screenshot of the QLQ (Step 6)

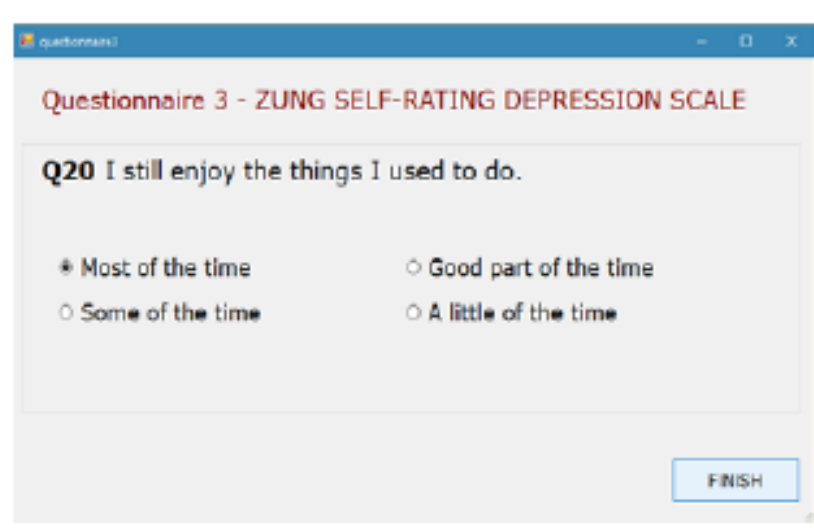

Fig. 7. Screenshot of the QLQ (Step 7) 


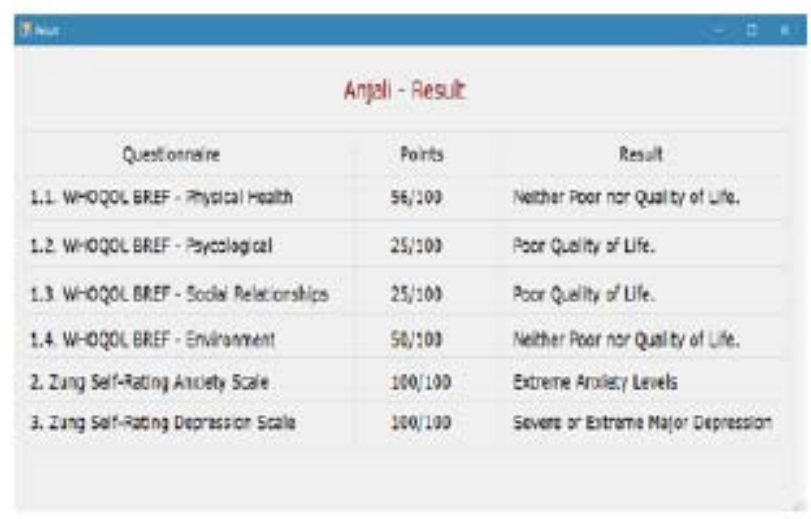

Fig. 8. Screenshot of the QLQ Result (Step 8)

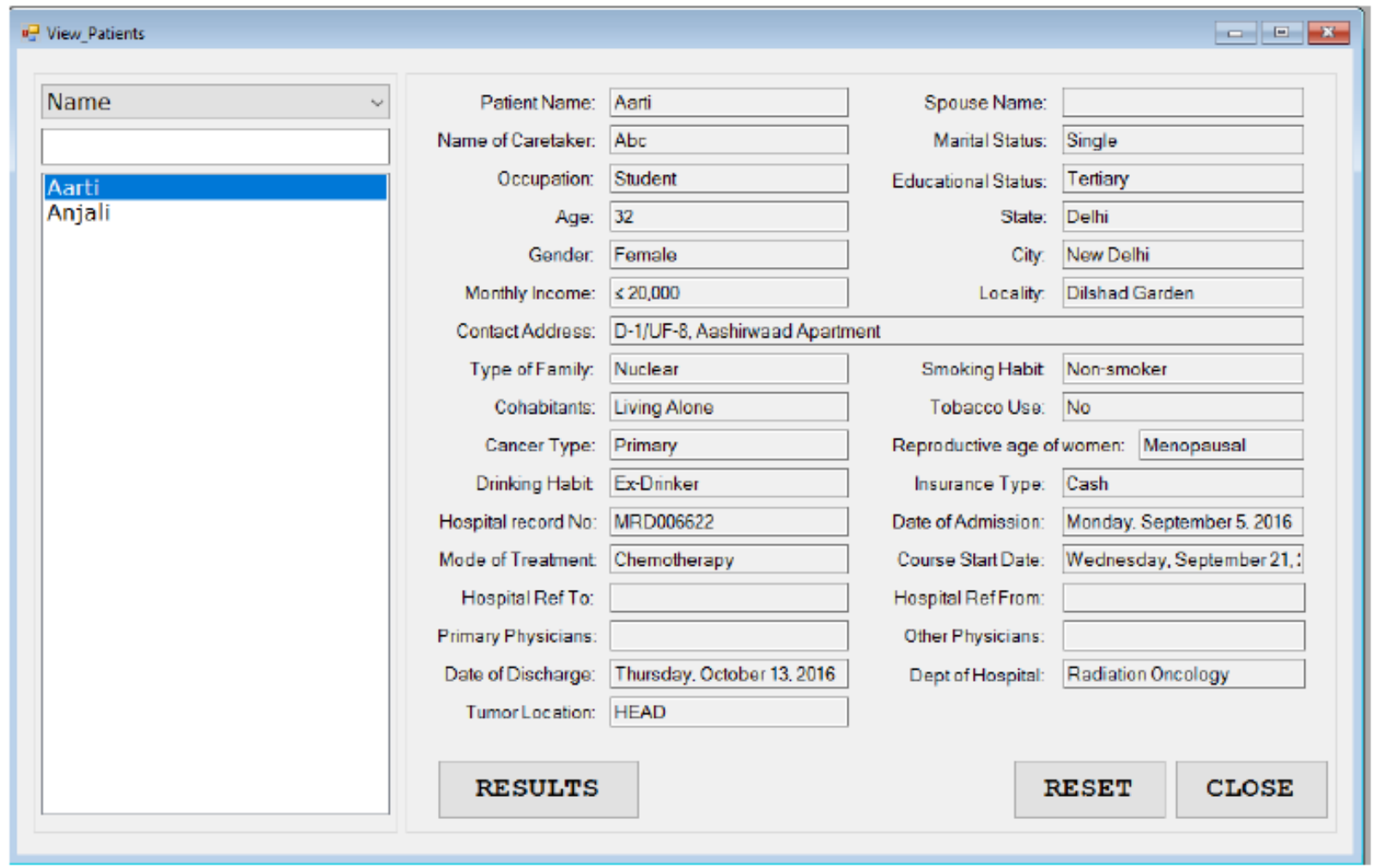

Fig. 9. Screenshot of the QLQ Saved data (Step 9)

\section{Discussion}

The time taken for QOL assessments in cancer patients manually was in a range : 30-59 (minutes). This required patience from the patients, as well as, the physician or healthcare professional. Since, the healthcare professionals and the physicians are very busy in the department because of workload as it was being estimated that there were only 1 doctor on 2000 patients in India. So, this ratio had a great impact on the patient's QOL during chemotherapy or radiotherapy treatment. The proposed computerized QOL could solve this problem upto a greater extent that involves patient's interest, full involvement, and ability to answer every question freely. On the other hand, physicians by looking at the filled form could easily analyze the factor affecting the most to the domains of QOL during cancer treatment and could plan accordingly with lessen time. Computerized QOL assessments had several advantages. They provided more accurate results, and as such, represent a picture of patients' QOL 
Texila International Journal of Clinical Research

Volume 3, Issue 2, Dec 2016

more clearly. Additionally, the use of technology to facilitate the implementation of QOL assessments increased the efficiency and allowed utilization by a wider population. Several studies have demonstrated the reasons why the implementation of computerized QOL assessments was found acceptable by physicians. According to a study by Buxton et al., a touch-screen computerized system had advantages over paper-and-pencil QOL questionnaires, which included the ability to increase the font size for those with visual difficulties, reduce the number of missed items, decrease the need for paper, analyze the patient's information immediately, and easily store and retrieve the data ${ }^{[1]}$. Allenby et al. reported that electronic completion of QOL questionnaires reduced the potential of errors with after-survey data input and can reduce the length of time it took to administer the questionnaires ${ }^{[1]}$. Taenzer et al. found patients took an average of 8.6 minutes to complete an electronic version of the EORTC QLQ-C30 questionnaire as opposed to 11 minutes to complete its paper version. In another study by Velikova et al. examining the difference between the electronic version of the EORTC QLQC30 and its paper version, patients took less time to complete the touch-screen version (8.3 minutes) as opposed to the paper version (9.6 minutes) ${ }^{[1]}$. Bliven et al. stated that the Internet allowed for the collection of patient-reported data at multiple locations, at frequent time intervals, and at little cost ${ }^{[1]}$. The key advantage of shifting to computer-based patient record was the opportunity to strengthen the link between the hospital records and management information system so that resources uses and quality of care could be analyzed using hospital database which increases physician efficiency and reduce costs, as well as promote standardization of care. QOL measures must be easily implemented into the medical office routine, requiring that they be short, easy to interpret, and not require intricate training or scoring. These assessments need to be acceptable to the patients and be able to produce reports in real time without disrupting busy clinics. Because of time constraints, patients, physicians, and office staff are not always willing to incorporate something new into their clinical routine. Providing interpretations and recommendations about available resources and the score may be helpful rather than just providing the physician with a functional status score.

Even though QOL information is clinically valuable, physicians often view it as providing "soft" data that does not permit "hard" measurement such as that obtained in the laboratory. Barriers to implementation could also arise due to the types of questions asked. Questions that are too personal, sensitive, or irrelevant are more likely to be omitted by respondents. For example, some questionnaires ask respondents about their careers. Pijls-Johannesma et al. reported that such questions are irrelevant in the elderly population, as many respondents were retired ${ }^{[1]}$.

\section{Conclusion}

In conclusion, it was observed that QOL assessment tools through computer assessments, had several advantages and were beneficial for cancer patients. The implementation of QOL assessments into clinical practice for cancer treatment has a high potential to benefit patients. Health-related quality of life has increasingly been an important factor to consider in the holistic treatment of cancer patients, and by providing accurate insights into anxiety, depression, and QOL through self-reported questionnaires, physicians would be better able to make treatment decisions. Technologies could provide a highly efficient and accurate means of implementing QOL assessments so that they can help a wider range of cancer patients. The advantages of implementing QOL tools are further supported by studies comparing intervention groups and controls, which have found that the intervention groups received more counseling and meaningful discussion with physicians. The information and advice gained in these counseling sessions have several benefits. In addition to learning about cancer, patients gain an understanding of how to best use the advice they received to improve the quality of their own lives. Because QOL instruments provide accurate assessments of the well-being and functionality of patients, utilizing them in clinical practice would significantly benefit patients and provide them with insights into their own care. This accurate, reliable and efficient method of computer based system generates the patient's QOL report and record as the patient register in and out of the hospital to ensure efficient outcome that will lessen time consuming. The development of 
electronic version of QLQ for measuring QOL of cancer patients which could enhance support to both the physicians and patient for better self-management, and improved adherence with chemotherapy and radiotherapy treatment. If successful, this intervention of electronic QLQ will be able to demonstrate and generate effects of Cancer and cancer treatment related effects on QOL of cancer patients that can be formally engaged to improve clinical outcomes.

Computerized version of QLQ could prove useful for evaluating cancer patients' experiences with hospitals that included the most important aspects regarding both inpatient and outpatient hospital care from the patient perspective. Eliciting feedback from patients helps healthcare providers to identify potential areas for improvement, which in turn can increase the quality of healthcare.

\section{Future directions}

Future studies involving the QOL of cancer patients should examine emerging science surrounding the implementation of QOL instruments, such as computerized versions. In order to create the most reliable and user-friendly application, potential users of the software should be included from the beginning to determine where possible problems might lie. Careful selection of the QOL assessment(s) should be considered with each population being examined to provide a reliable and valid patient-reported outcome.

\section{Limitations of the study}

1. The study did not implement the computerized version for the assessment, in fact, it was the outcome of the study, as the patients faced the difficulty while filling up the manual questionnaire.

2. To validate the electronic version, further cross-sectional study with large pool of sample size is required.

\section{Footnotes}

Author's Contribution: 1- acquisition of data; 2- analysis and interpretation of data; 3- drafting of the manuscript; 4- critical revision of the manuscript for important intellectual content; 5- statistical analysis; 6- administrative, technical and material supports, and software of QLQ- By Mr. Anant Gupta (Aanmaak Technologies (OPC) Pvt. Ltd., New Delhi).

Funding/Support: Self-funded.

\section{Acknowledgement}

The present study is a part of the research programme, "Assessment of Quality of Life of Adult Cancer Patients during inpatient Chemotherapy Treatment (CT), or outpatient Radiotherapy Treatment (RT) in a tertiary care hospital of New Delhi, India”. This research was supportedand assisted by at:

Department of Medical Oncology and Radiation Oncology, Dr. B.L. Kapur Memorial Hospital, New Delhi, India.

Under the Supervision of

Dr. S. Hukku (H.O.D. of Radiation Oncology Department),

Dr. B.L. Kapur Memorial Hospital,

New Delhi, India.

Dr. Amit Agarwal (H.O.D. of Medical Oncology Department),

Dr. B.L. Kapur Memorial Hospital,

New Delhi, India.

Under the Guidance of:

Dr. Renita Bhamrah (Texila American University),

E-mail:darpreetrenita@gmail.com 
Texila International Journal of Clinical Research

Volume 3, Issue 2, Dec 2016

\section{References}

[1]. Perry, S., Kowalski, T. L., \& Chang, C.-H. (2007). Quality of life assessment in women with breast cancer: benefits, acceptability and utilization. Health and Quality of Life Outcomes, 5, 24.

http://doi.org/10.1186/1477-7525-5-24

[2]. WHOQOL Group. (1998). Development of the World Health Organization WHOQOLBREF quality of life assessment. Psychol Med, 28:551-8.

[3]. Zung, W. W. K. (1971). A rating instrument for anxiety disorders. Psychosomatics, 12(6), 371-379. Available at: https://psychology-tools.com/zung-anxiety-scale/

[4]. Zung, W. W. K. (1965). A Self-Rating Depression Scale. (1965). Arch Gen Psychiatry, 12: 63-70. Available at: https://psychology-tools.com/zung-depression-scale/ 


\title{
Sexual Health, Suicidal Ideation and weight changes in Cancer patients during Chemotherapy and Radiotherapy Treatment
}

\author{
Article by Kavita Gupta \\ Ph.D. Clinical Research, Texila American University, India \\ E-mail: 16kavitagupta@texilaconnect.com
}

\begin{abstract}
Background: During chemotherapy or radiotherapy sessions, cancer patients often go through a series of physical, psychological, social and environmental changes that affects the QOL of the patients. While assessing for the anxiety and depression scores affecting the QOL, maximum of the patients left the question of sexual activity, suicidal ideation and weight issues unanswered.

Objectives: The present paper focused on the issue of sexual activity, suicidal ideation, and weight changes during chemotherapy or radiotherapy sessions while determining the anxiety and depression scores for QOL of cancer patients during the treatment regimen.

Methods: It was a cross-sectional, descriptive, hospital based evaluation study. Total duration of the study was 5 months (December 2015- April 2016), conducted in Medical and Radiation Oncology department of Dr. B.L. Kapur Memorial Hospital, New Delhi, India. A convenience sample of 60 patients (Chemotherapy group $(n=30)$ and Radiotherapy group $(n=30))$ with cancer was selected. Data was obtained through direct interview, using validated Psychological intervention tool in the form of Questionnaire: WHOQOL-Bref Questionnaire, Zung Self-Rating Anxiety scale and Zung Self-Rating Depression scale, which was further managed through a statistical program, using appropriate statistical tests.

Results: A total of 60 cancer patients were included in the study in which Chemotherapy group consisted of 30n cancer patients and Radiotherapy group consisted of 30n cancer patients. In the study, 6(20\%) were males and 24(80\%) were females in the chemotherapy group, and, 15(50\%) were males and $15(50 \%)$ were females in the radiotherapy group. Majority of the patients 32(53.34\%) were in the age range of 46-60 years.

Conclusion: It could be concluded from the present study that in Indian scenario, cancer patients felt sexual activity and suicidal ideation as a social stigma which is further accompanied by hesitation. Moreover, maximum patients observed stable weight and some patients observed increase in weight during chemotherapy sessions.
\end{abstract}

Keywords: Cancer, Chemotherapy, Radiotherapy, Quality of Life, missing values, sexuality, suicidal ideation, weight increased, weight stability.
Abbreviations
QOL : Quality of life
QLQ : Quality life Questionnaire
WHOQOL-Bref : World Health Organization Quality of life assessment-a short brief version
ZSAS $\quad$ : Zung Self-Rating Anxiety scale
ZSDS $\quad$ : Zung Self-Rating Depression scale 
Texila International Journal of Clinical Research

Volume 3, Issue 2, Dec 2016

$\begin{array}{ll}\text { CT } & : \text { Chemotherapy Treatment } \\ \text { RT } & : \text { Radiotherapy Treatment } \\ \text { DOM } & : \text { Domain } \\ \text { TPA } & : \text { Third Party Administrator } \\ \text { FNAC } & : \text { Sine needle aspiration cytology } \\ \text { SD } & \end{array}$

\section{Introduction}

It is important to study QOL aspects in cancer patients because of chronic nature of the disease, regular follow-up by the patient, different types of treatments given (radiation, surgery and chemotherapy), influence on sexual life, suicidal ideation and self-identity of cancer patients ${ }^{[1]}$. Cancer treatment, especially chemotherapy, creates changes in the patient's body that affects sexual desire, sexual functioning, and emotional relationships ${ }^{[2]}$. Human sexuality is an important aspect of well-being that could be altered significantly by cancer and its related treatment ${ }^{[2]}$. Because cancer is not considered a chronic illness until recently, sexuality, suicidal ideation, and weight changes issue usually is not addressed by nurses or other health care professionals ${ }^{[3]}$. Although healthy women also experience physiological changes leading to menopause, these changes occurred gradually leaving them sexually active 5 to 10 years longer and with fewer problems in sexual functioning ${ }^{[2]}$. Studies showed that breast cancer patients experienced sexual problems soon after treatment, and continue in follow-up ${ }^{[2]}$. Research also described the normal decline in sexuality among healthy men and women as they age ${ }^{\text {[2] }}$. Furthermore, relationship problems occurred when couples experienced sexual problems, sometimes threatening their attachment ${ }^{[2]}$. These problems could be anticipated and addressed through the physician's communication with their patient ${ }^{[2]}$. Other solutions included effective psychologic and emotional counseling and pharmaceutical and over the counter assistance for dealing with the related issues $^{[2]}$. It is essential that nurses and healthcare professionals have the appropriate knowledge, skill, and attitudes to address the oncology patient's concerns about sexuality effectively ${ }^{[3]}$. Using Annon's PLISSIT (permission, limited information, specific suggestions, and intensive therapy) model of sexual assessment, the healthcare professionals could learn how to assess a patient's sexual concerns, thus breaking the silence ${ }^{[3]}$.

\section{Aims}

The main objectives of the present study was:

i. To evaluate and determine the missing values of sexual health, suicidal ideation, and weight changes in the WHOQOL-Bref, Zung Self-rating Anxiety Scale (ZSAS) that affected overall QOL in cancer patients during Chemotherapy or Radiotherapy treatment regimen.

\section{Patients and methods}

The proposed study was conducted in accordance and adherence to the Ethical Guidelines and Procedures. Special care of the potential risks due to emotional distress was taken care of so that the dignity of the subject was not harmed. The authorized Ethical approval from the IRB and Ethical committee of Dr. B.L. Kapur Memorial Hospital, New Delhi, with Ref. No.: IRB/AARCE/5/DEC/2015/1, dated December $7^{\text {th }}$, 2015, was obtained to carry out the research study. Thereafter, the patients and their caregivers were approached in the inpatient as well as, outpatient clinic, where the purpose of the study was explained and they were invited to participate. Patients who agreed to 
participate were asked to sign an Informed Consent Form followed by the implementation of the Structured and Validatedinstrumental tool of WHOQOL-Bref, Zung Self-Rating Anxiety Scale (ZSAS), and Zung Self-Rating Depression Scale (ZSDS) in the form of questionnaire which lasted for approximately 25-60 minutes. The RESEARCH DESIGN of the proposed approved study protocol included 30n cancer patients undergoing Chemotherapy Treatment and 30n cancer patients undergoing Radiotherapy Treatment session. The inclusion criteria for the approved study were the patients with Breast cancer, sub-sites of head and neck tumors (e.g., nasopharyngeal, thyroid cancer, and parotidtumors), aged 18years or older, Clinically diagnosed and confirmed by biopsy or FNAC, Undergoing/during the treatment sessions ( $\geq 2$ cycles and $\leq 6$ for Chemotherapy treatment, and $\geq 10$ cycles and $\leq 30$ cycles for Radiotherapy treatment, Voluntarily agreed to join the study, and aware of diagnosis and predicted prognosis. However, the study excluded patients with Inadequate clinical condition (ambulatory and terminally ill patients) who were unable to respond to an interview, had difficulty in understanding the questionnaire or communicating, were serious and didn't give consent, had a history of psychiatric disorder. Moreover, the study did not include the dosage of chemotherapy drugs and irradiation treatment. Eligible patients were identified through an institutional database or by referring physicians and were approached at their simulation appointment.

\section{Results}

\section{(a) Data collection}

Treatment-related symptoms were assessed by using a series of interviews through standard questionnaires of WHOQOL-Bref, the core questionnaire, followed by Zung Self-Rating Anxiety Scale (ZSAS) and Zung Self Rating Depression Scale (ZSDS) Questionnaires. These questionnaires have been proven to have good validity and reliability properties, cross-culturally accepted instrument to measure Quality of Life in cancer patients and are publicly available for scholar research purposes ${ }^{[5,6,7]}$. The questionnaire was provided in a language that the patient could understand easily (English / Hindi) followed by face-to-face interview of the patient who was either undergoing chemotherapy or radiotherapy treatment sessions.

Parts of the Record Card:

i. Personal information form:This first part contained patient information. The form was prepared, based on the literature. It contained age, gender, qualification, marital status, family type, Occupation, data on financial income and site of tumor location ${ }^{[5]}$.

ii. WHOQOL-Bref Questionnaire: This is an abbreviated version of the instrument WHOQOL-100. It consisted of 26 questions, being two about quality of life in general and other 24 representing each of the facets that made up the original instrument ${ }^{[5]}$. The questions were organized in 4 domains:

a. Physical domain (DOM1): It included7 questions pertaining to sleep, energy, mobility, the extent to which pain prevents performance of necessary tasks, the need for medical treatment to function in daily life, level of satisfaction with their capacity for work ${ }^{[5]}$.

b. Psychological domain (DOM2): It included6 questions pertaining to the ability to concentrate, selfesteem, body image, spirituality i.e. the extent to which they feel their life is meaningful, the frequency of positive or negative feelings i.e. blue mood, despair, anxiety, depression ${ }^{[5]}$.

c. Social domain (DOM3): It included 3 questions pertaining to satisfaction with personal relationships, social support systems and sexual satisfaction ${ }^{[5]}$.

d. Environmental domain (DOM4): It included 8 questions related to safety and security, home and physical environment satisfaction, finance i.e. does the respondent have enough money to meet their needs, health/social care availability, information and leisure activity accessibility and transportation satisfaction ${ }^{[5]}$.

Equations for computing domain raw scores:

Domain 1 (Physical) score = (6- Q3) + (6-Q4) + Q5 + Q6 + Q7 + Q8 + Q9

Domain 2 (Psychological) score = Q10 + Q11 + Q12 + Q13 + Q14 + (6-Q15) 
Texila International Journal of Clinical Research

Volume 3, Issue 2, Dec 2016

Domain 3 (Social) score $=$ Q16 + Q17 + Q18

Domain 4 (Environmental) score = Q19 + Q20 + Q21 + Q22 + Q23 + Q24 + Q25 + Q26 ${ }^{[5]}$.

In addition to the 4 domains, the WHOQOL-Bref included two stand-alone questions, one pertaining to the respondents' rated QOL, and one related to their Satisfaction with Health were analyzed separately ${ }^{[5]}$. The score of each question ranged from 1 to 5 on a 5 point likert scale and higher scores indicated a better evaluation. Raw scores of the respective domains were then transformed from 0-100 with the lowest score of zero and the highest score of 100according to the accepted guidelines ${ }^{[5]}$.

iii.ZSAS: Zung Self-Rating Anxiety scale quality life questionnaire is a likert scale format (scoring on 1 to 4 scale) that was built by a psychiatrist, William W. K. Zung to measure the rate of anxiety. The scale consisted of 20 self-reported items with 15 questions of increasing anxiety level and 5 questions of decreasing anxiety level (Q. no. 5, 9, 13, 17, 19) ${ }^{[6]}$. Scores for each question ranged from 1 to 4 and higher scores indicated severe anxiety level. The raw scores were counted up and multiplied by 1.25 to reach a standardized score, according to the instructions that accompanied the scale ${ }^{[6]}$. The ZSAS Index score followed the crierion: Normal Range (20-44); Mild to Moderate Anxiety level (45-59); Marked to Severe Anxiety level (60-74); Extreme Anxiety level (75-80) ${ }^{[6]}$.

iv.ZSDS: Zung Self-Rating Depression scale quality life questionnaire is also a 20 items short selfadministered survey that was designed by William W. K. Zung to assess the level of four common characteristics of depression for patients: the pervasive effect, the physiological equivalents, other disturbances, and psychomotor activities. There were ten positively worded and ten negatively worded questions. Each question was scored on a scale of 1-4 (a little of the time, some of the time, good part of the time, most of the time) ${ }^{[7]}$. The higher scores indicated severe depression level. The raw scores were counted up and multiplied by 1.25 to reach a standardized score, according to the instructions that accompanied the scale ${ }^{[7]}$. The ZSDS Index score followed the crierion: Normal Range $(<50)$; Mild Depression level $(<60)$; Moderate Depression level $(<70)$; Extreme Depression level $(>70){ }^{[7]}$.

\section{(b) Statistical analyses}

The database and statistical analysis was performed by using SPSS v.17 software. The independent variables analyzed were Socio-demographic characteristics (sex/gender, age, education level, marital status, employment type, income level (per annum), job background, local residence), Clinical characteristics (smoking habit, drinking habit, tobacco use, health insurance, tumor type \& location, metastasis involved, corresponding cycle number for both chemotherapy and radiotherapy treatment); Anxiety and Depression of the patients as assessed by ZSAS and ZSDS respectively. The dependent variables included: subscale and overall QOL scores, and Health satisfaction as measured by WHOQOLBref Questionnaire (Tool/instrument). Descriptive statistics computation techniques were applied to the discrete and continuous data. Measures such as mean, standard deviation, minimum and maximum range were developed from the continuous data. Relative frequency was calculated for discrete data. Mean with Standard deviation (SD) was used to summarize the age of patients. Chi-square test was performed to assess the effect of different sociodemographic factors and clinical characteristics on the QOL of the cancer patients. Student's $t$-test was used to compare sample means for study variables (anxiety, depression and QOL). Bivariate analysis was performed to assess the predictors of QOL. Based on the survey/ research, Pearson Correlation coefficient test denoted by $r$ was calculated to assess the particular characteristic symptomatic function of anxiety and depression instrument that significantly affected the QOL domains, i.e., either positively or negatively. Paired t-test was used to compare difference between score means of different domains. A $p$-value $<0.05$ was considered as statistical significant. 


\section{Socio-Demographic characteristics of the patients}

Table 1. Socio-demographic characteristics in the form of Frequency and percentage of variables of the patients and correlation in the two groups, i.e., Chemotherapy and Radiotherapy

(All tests were performed using Pearson $\chi 2$ test for association analysis)

\begin{tabular}{|c|c|c|c|c|c|c|}
\hline \multirow[t]{2}{*}{ S.No } & \multirow[t]{2}{*}{ Variables } & \multirow[t]{2}{*}{ Parameters } & \multicolumn{2}{|c|}{ Chemotherapy } & \multicolumn{2}{|c|}{ Radiotherapy } \\
\hline & & & $\begin{array}{l}\text { Frequency } \\
\text { (n) }\end{array}$ & $\begin{array}{l}\text { Percentage } \\
\text { (\%) }\end{array}$ & $\begin{array}{l}\text { Frequency } \\
\text { (n) }\end{array}$ & $\begin{array}{l}\text { Percentage } \\
\text { (\%) }\end{array}$ \\
\hline 1. & Gender & $\begin{array}{l}\text { a. Male } \\
\text { b. Female }\end{array}$ & $\begin{array}{l}6 \\
24\end{array}$ & $\begin{array}{l}20.0 \% \\
80.0 \%\end{array}$ & $\begin{array}{l}15 \\
15\end{array}$ & $\begin{array}{l}50.0 \% \\
50.0 \%\end{array}$ \\
\hline 2. & Age & $\begin{array}{l}\text { a. } 18-30 \text { years } \\
\text { b. } 30 \\
\text { c. } 46 \\
\text { d. } 60\end{array}$ & $\begin{array}{l}5 \\
17 \\
8 \\
\end{array}$ & $\begin{array}{l}16.7 \% \\
56.7 \% \\
26.7 \% \\
\end{array}$ & $\begin{array}{l}6 \\
15 \\
9 \\
\end{array}$ & $\begin{array}{l}20.0 \% \\
50.0 \% \\
30.0 \% \\
\end{array}$ \\
\hline 3. & $\begin{array}{l}\text { Marital } \\
\text { status }\end{array}$ & $\begin{array}{l}\text { a. Unmarried } \\
\text { b. Married } \\
\text { c. Widow } \\
\text { d. Divorced/ } \\
\quad \text { Legally } \\
\text { separated } \\
\text { e. Others }\end{array}$ & 30 & $100.0 \%$ & $\begin{array}{l}1 \\
28 \\
1\end{array}$ & $\begin{array}{l}3.3 \% \\
93.3 \% \\
3.3 \%\end{array}$ \\
\hline 4. & $\begin{array}{l}\text { Educational } \\
\text { status }\end{array}$ & $\begin{array}{l}\text { a. Illiterate } \\
\text { b. Literate }\end{array}$ & 1 & $3.3 \%$ & 2 & $6.7 \%$ \\
\hline & & $\begin{array}{l}\text { i. Primary } \\
\text { ii. Secondary } \\
\text { iii.Tertiary }\end{array}$ & $\begin{array}{l}4 \\
10 \\
15\end{array}$ & $\begin{array}{l}13.3 \% \\
33.3 \% \\
50.0 \%\end{array}$ & $\begin{array}{l}5 \\
11 \\
12\end{array}$ & $\begin{array}{l}16.7 \% \\
36.7 \% \\
40.0 \%\end{array}$ \\
\hline 5. & Occupation & $\begin{array}{l}\text { a. Service } \\
\text { b. Business } \\
\text { c. Housewife } \\
\text { d. Freelancers } \\
\text { e. Pensioners } \\
\text { f. Domestic } \\
\quad \text { duties } \\
\text { g. Cultivation }\end{array}$ & $\begin{array}{l}3 \\
3 \\
21 \\
3\end{array}$ & $\begin{array}{l}10.0 \% \\
10.0 \% \\
70.0 \% \\
10.0 \%\end{array}$ & $\begin{array}{l}10 \\
2 \\
11 \\
1 \\
4 \\
1 \\
1\end{array}$ & $\begin{array}{l}33.3 \% \\
6.7 \% \\
36.7 \% \\
3.3 \% \\
13.3 \% \\
3.3 \% \\
3.3 \%\end{array}$ \\
\hline 6. & $\begin{array}{l}\text { Type of } \\
\text { family }\end{array}$ & $\begin{array}{l}\text { a. Nuclear } \\
\text { b. Joint }\end{array}$ & $\begin{array}{l}16 \\
14\end{array}$ & $\begin{array}{l}53.3 \% \\
46.7 \%\end{array}$ & $\begin{array}{l}9 \\
21\end{array}$ & $\begin{array}{l}30.0 \% \\
70.0 \%\end{array}$ \\
\hline 7. & Cohabitants & $\begin{array}{l}\text { a. Living } \\
\text { alone } \\
\text { b. Living with } \\
\text { partner } \\
\text { c. Living with } \\
\text { partner and } \\
\text { children }\end{array}$ & $\begin{array}{l}15 \\
1\end{array}$ & $\begin{array}{l}50.0 \% \\
3.3 \%\end{array}$ & $\begin{array}{l}4 \\
5\end{array}$ & $\begin{array}{l}13.3 \% \\
16.67 \%\end{array}$ \\
\hline
\end{tabular}


Texila International Journal of Clinical Research

Volume 3, Issue 2, Dec 2016

\begin{tabular}{|c|c|c|c|c|c|c|}
\hline & & $\begin{array}{l}\text { d. Living with } \\
\text { children }\end{array}$ & 14 & $46.7 \%$ & 21 & $70.0 \%$ \\
\hline 8. & $\begin{array}{l}\text { Annual } \\
\text { income }\end{array}$ & $\begin{array}{l}\text { a. NA } \\
\text { b. } \leq 20 \\
\text { c. } 20 \\
\text { d. } 30 \\
\text { e. } 41 \\
\text { f. } \geq 84\end{array}$ & 22 & $73.3 \%$ & 11 & $63.3 \%$ \\
\hline 9. & $\begin{array}{l}\text { Place of } \\
\text { residence }\end{array}$ & $\begin{array}{l}\text { a. Small town } \\
\text { b. Big town }\end{array}$ & $\begin{array}{l}1 \\
29\end{array}$ & $\begin{array}{l}3.3 \% \\
96.7 \%\end{array}$ & $\begin{array}{l}8 \\
22\end{array}$ & $\begin{array}{l}26.7 \% \\
73.3 \%\end{array}$ \\
\hline
\end{tabular}

Clinical characteristics of the patients

Table 2. Clinicalcharacteristics in the form of Frequency and percentage of variables of the patients and correlation in the two groups, i.e., Chemotherapy or Radiotherapy

(All tests were performed using Pearson $\chi 2$ test for association analysis).

\begin{tabular}{|c|c|c|c|c|c|c|}
\hline \multirow[t]{2}{*}{ S.No. } & \multirow[t]{2}{*}{ Variables } & \multirow[t]{2}{*}{ Parameters } & \multicolumn{2}{|c|}{ Chemotherapy } & \multicolumn{2}{|c|}{ Radiotherapy } \\
\hline & & & $\begin{array}{l}\text { Frequency } \\
\text { (n) }\end{array}$ & $\begin{array}{l}\text { Percentage } \\
\text { (\%) }\end{array}$ & $\begin{array}{l}\text { Frequency } \\
\text { (n) }\end{array}$ & $\begin{array}{l}\text { Percentage } \\
(\%)\end{array}$ \\
\hline 1. & $\begin{array}{l}\text { Smoking } \\
\text { habit }\end{array}$ & $\begin{array}{l}\text { a. Non-smoker } \\
\text { b. Ex-smoker }\end{array}$ & $\begin{array}{l}27 \\
3 \\
\end{array}$ & $\begin{array}{l}90.0 \% \\
10.0 \% \\
\end{array}$ & $\begin{array}{l}27 \\
4 \\
\end{array}$ & $\begin{array}{l}90.0 \% \\
10.0 \% \\
\end{array}$ \\
\hline 2. & $\begin{array}{l}\text { Drinking } \\
\text { habit }\end{array}$ & $\begin{array}{l}\text { a. Non-drinker } \\
\text { b. Ex-drinker }\end{array}$ & 30 & $100.0 \%$ & $\begin{array}{l}26 \\
4 \\
\end{array}$ & $\begin{array}{l}86.7 \% \\
13.3 \% \\
\end{array}$ \\
\hline 3. & $\begin{array}{l}\text { Tobacco } \\
\text { use }\end{array}$ & $\begin{array}{l}\text { a. Yes } \\
\text { b. No }\end{array}$ & $\begin{array}{l}1 \\
29 \\
\end{array}$ & $\begin{array}{l}3.3 \% \\
96.7 \% \\
\end{array}$ & $\begin{array}{l}7 \\
23 \\
\end{array}$ & $\begin{array}{l}23.3 \% \\
76.7 \% \\
\end{array}$ \\
\hline 4. & $\begin{array}{l}\text { Health } \\
\text { insurance }\end{array}$ & $\begin{array}{l}\text { a. Yes } \\
\text { b. No }\end{array}$ & $\begin{array}{l}23 \\
7 \\
\end{array}$ & $\begin{array}{l}76.7 \% \\
23.3 \% \\
\end{array}$ & $\begin{array}{l}19 \\
11\end{array}$ & $\begin{array}{l}63.3 \% \\
36.7 \% \\
\end{array}$ \\
\hline 5. & $\begin{array}{l}\text { Type of } \\
\text { health } \\
\text { insurance }\end{array}$ & $\begin{array}{l}\text { a. Government } \\
\text { medically } \\
\text { insured } \\
\text { b. TPA } \\
\text { c. Cash }\end{array}$ & $\begin{array}{l}10 \\
13 \\
7\end{array}$ & $\begin{array}{l}33.3 \% \\
43.3 \% \\
23.3 \%\end{array}$ & $\begin{array}{l}12 \\
7 \\
11\end{array}$ & $\begin{array}{l}40.0 \% \\
23.3 \% \\
36.7 \%\end{array}$ \\
\hline 6. & $\begin{array}{l}\text { Support by } \\
\text { charity } \\
\text { organizatio } \\
n\end{array}$ & $\begin{array}{l}\text { a. Yes } \\
\text { b. No }\end{array}$ & 30 & $100.0 \%$ & 30 & $100 \%$ \\
\hline 7. & $\begin{array}{l}\text { Cancer } \\
\text { tumor } \\
\text { location }\end{array}$ & a. Ca Breast & 23 & $76.7 \%$ & 10 & $33.3 \%$ \\
\hline
\end{tabular}


Texila International Journal of Clinical Research Volume 3, Issue 2, Dec 2016

\begin{tabular}{|c|c|c|c|c|c|c|}
\hline & & $\begin{array}{l}\text { b. Ca Head } \\
\text { c. Ca Neck }\end{array}$ & $\begin{array}{l}1 \\
6\end{array}$ & $\begin{array}{l}3.3 \% \\
20.0 \%\end{array}$ & $\begin{array}{l}5 \\
15\end{array}$ & $\begin{array}{l}16.7 \% \\
50.0 \%\end{array}$ \\
\hline 8. & $\begin{array}{l}\text { Disease } \\
\text { acceptance }\end{array}$ & $\begin{array}{l}\text { a. Yes } \\
\text { b. No }\end{array}$ & $\begin{array}{l}23 \\
7 \\
\end{array}$ & $\begin{array}{l}76.7 \% \\
23.3 \% \\
\end{array}$ & $\begin{array}{l}15 \\
15 \\
\end{array}$ & $\begin{array}{l}50.0 \% \\
50.0 \% \\
\end{array}$ \\
\hline 9. & $\begin{array}{l}\text { Reproductiv } \\
\text { e age of } \\
\text { women }\end{array}$ & $\begin{array}{l}\text { a. Pre- } \\
\text { menopausal } \\
\text { b. Menopausal }\end{array}$ & $\begin{array}{l}10 \\
14 \\
\end{array}$ & $\begin{array}{l}33.3 \% \\
46.7 \% \\
\end{array}$ & $\begin{array}{l}9 \\
6 \\
6\end{array}$ & $\begin{array}{l}30.0 \% \\
20.0 \% \\
\end{array}$ \\
\hline 10. & Cancer type & $\begin{array}{l}\text { a. Primary } \\
\text { cancer } \\
\text { b. Recurrent } \\
\text { cancer } \\
\end{array}$ & 30 & $100.0 \%$ & 30 & $100.0 \%$ \\
\hline 11. & $\begin{array}{l}\text { Co- } \\
\text { existence of } \\
\text { metastasis }\end{array}$ & $\begin{array}{l}\text { a. Yes } \\
\text { b. No }\end{array}$ & $\begin{array}{l}8 \\
22\end{array}$ & $\begin{array}{l}26.7 \% \\
73.4 \%\end{array}$ & 30 & $100.0 \%$ \\
\hline 12. & $\begin{array}{l}\text { Chemothera } \\
\text { py's cycle } \\
\text { during the } \\
\text { interview of } \\
\text { QLQ }\end{array}$ & $\begin{array}{l}\text { a. } 1^{\text {st }} \\
\text { b. } 2^{\text {nd }} \\
\text { c. } 3^{\text {rd }} \\
\text { d. } 4^{\text {th }} \\
\text { e. } 5^{\text {th }} \\
\text { f. } 6^{\text {th }}\end{array}$ & $\begin{array}{l}2 \\
15 \\
6 \\
6 \\
1\end{array}$ & $\begin{array}{l}6.7 \% \\
50.0 \% \\
20.0 \% \\
20.0 \% \\
3.3 \%\end{array}$ & & \\
\hline 13 & $\begin{array}{l}\text { Radiotherap } \\
\text { y's cycle } \\
\text { during the } \\
\text { interview of } \\
\text { QLQ }\end{array}$ & $\begin{array}{l}\text { a. } 10^{\text {th }}-15^{\text {th }} \\
\text { b. } 16^{\text {th }}-20^{\text {th }} \\
\text { c. } 21^{\text {st }}-25^{\text {th }} \\
\text { d. } 26^{\text {th }} 30^{\text {th }}\end{array}$ & & & $\begin{array}{l}21 \\
3 \\
1 \\
\end{array}$ & $\begin{array}{l}16.7 \% \\
\\
\\
\\
70.0 \% \\
10.0 \% \\
3.3 \% \\
\end{array}$ \\
\hline
\end{tabular}


Texila International Journal of Clinical Research

Volume 3, Issue 2, Dec 2016

Response pattern and missing items for each item in WHOQOL Bref, ZSAS, and ZSDS in both Chemotherapy $(n=30)$ and Radiotherapy $(n=30)$ group

Table 3. Missing values while assessing for QOL of cancer patients for chemotherapy group $(\mathrm{n}=30)$ and radiotherapy group $(\mathrm{n}=30)$.

\begin{tabular}{|l|l|l|l|l|l|l|}
\hline \multirow{2}{*}{ Item number } & \multicolumn{2}{l|}{ Chemotherapy } & \multicolumn{2}{l|}{ Radiotherapy } \\
\cline { 2 - 7 } & $\begin{array}{l}\text { Missingn } \\
(\%)\end{array}$ & $\begin{array}{l}\text { Mean } \\
\text { Score }\end{array}$ & SD & $\begin{array}{l}\text { Missingn } \\
(\%)\end{array}$ & $\begin{array}{l}\text { Mean } \\
\text { Score }\end{array}$ & SD \\
\hline $\begin{array}{l}\text { WHOQOL-BREF Scale: } \\
\text { Social Domain }\end{array}$ & & & & & & \\
Q 18. Sexual Activity & $30(100 \%)$ & 0.00 & 0.00 & $28(93.3 \%)$ & 0.06 & 0.25 \\
\hline ZSDS Scale: & & & & & & \\
Q 6. Sexual Activity & $30(100 \%)$ & 0.00 & 0.00 & $30(100 \%)$ & 0.00 & 0.00 \\
Q 7. Weight Loss & $14(46.7 \%)$ & 3.18 & 1.08 & 0.00 & 0.00 & 0.00 \\
Q 19. Suicidal Ideation & $30(100 \%)$ & 0.00 & 0.00 & $29(96.7 \%)$ & 1.00 & \\
\hline ZSDS Scale *: & & & & & & \\
\hline Q 7. Weight Constant* & $9(30.0 \%)$ & 2.55 & 1.23 & 0.00 & 0.00 & 0.00 \\
\hline Q 7. Weight Increased* & $5(16.66 \%)$ & 2.20 & 0.44 & 0.00 & 0.00 & 0.00 \\
\hline
\end{tabular}

The above table depicted that approximately all of the patients in both the treatment groups did not opt for the question of sexual activity and suicidal ideation. Moreover, weight loss was observed in 16 (53.33\%) patients undergoing chemotherapy; and 30(100\%) in the patients undergoing radiotherapy. On the other hand, 9(30.0\%) patients observed constant/stable weight, and 5(16.66\%) patients observed increase in weight during chemotherapy sessions.

\section{Discussion}

The present study determined that sexual activity factor in both the WHOQOL-Bref and ZSDS questionnaire was left unanswered by approximately most of the cancer patients in chemotherapy as well as, radiotherapy group. However, 2(93.3\%) patients with a mean score of 0.06 and SD of 0.25 answered the question of sexual activity in the social domain of WHOQOL-Bref but did not attempt it in the ZSDS questionnaire which depicted that this question was somewhere upsetting. Sexuality still seems to be tabooed among cancer patients, cancer patients being reluctant to talk about their sexual life, as they are afraid this might compromise their relationship. Older Polish people, especially over 65 years of age, regard questions pertaining to sexuality as "upsetting". On the other hand, it was observed that $9(2.55 \pm 1.23)$ patients observed constant weight and 5(2.20 \pm 0.44$)$ patients observed increase in weight during the chemotherapy sessions for cancer treatment. Moreover, 1(3.3\%) patient answered question of suicidal ideation of ZSDS in the radiotherapy group.

Women were found to be more affected during the treatment regimen and that too in the chemotherapy group ${ }^{[1]}$. Moreover, a woman plays vital central role in the family as a wife, sister, mother, and daughter, but there was some amount of negligence toward a woman's health in Indian families ${ }^{[1]}$. Some kind of blushing nature and negligence toward self-health was also there in Indian women as compared with the western population ${ }^{[1]}$. As per Montazeri study, when a woman developed breast cancer, all family members might develop some sort of illness ${ }^{[1]}$. If we look at the other parts, in western countries generally the decision about the health of the woman were taken by the patient herself but in India, spouse, family background and family income played an important role in the healthcare decision making for the patient ${ }^{[1]}$. Therefore, marital status of the patient might not be ignored as a factor affecting QOL ${ }^{[1]}$. During and after receiving treatment for cancer, men of all ages, with early and advanced diseases, will have concerns and questions about sexuality and sexual activity. It is common for men with different types of cancer to struggle with their body image; have less desire for sexual intimacy and/or have a 
change in their ability to achieve or maintain an erection during sexual activity or a change in orgasm or climax. Detection of cancer brings many changes in the life of a patient. For some of them it is a life changing experience ${ }^{[4]}$.

\section{Conclusion}

Sexual problems, suicidal ideation are a widespread concern among patients and survivors, but there was much variation in experiences of communication about sexual issues, and many patients did not receive the information they need from their oncology providers. There were large differences in sexual function between patients who did and did not ask providers about sexual problems. Sexual health had yet to be fully integrated into oncology care, even for cancers involving sex organs. On the hand, suicidal ideation feeling was increased due to increased depression. Therefore, this factor was also found to be ignored which needs attention. Moreover, some patients observed stable weight and some observed increase in their weight which needs to be further evaluated. Sexuality encompasses much more than sex; it includes the physical, psychological, emotional and social aspects of sex. In the real world, the present issues discuused means how the cancer patient see himself, how does his partner view him, how didhe date after cancer, how didhe fulfill his need for sexual relationships after cancer and so much more.

\section{Future directions}

a) The present missing values needs to be tested in the cancer patients that would attend either inpatient or outpatient department in the clinic for chemotherapy or radiotherapy treatment regimen.

\section{Limitations of the study}

a) Small number of sample size with single-centered study.

\section{Footnotes}

Author's Contribution: 1- acquisition of data; 2- analysis and interpretation of data; 3- drafting of the manuscript; 4- critical revision of the manuscript for important intellectual content; 5- statistical analysis; 6- administrative, technical and material supports.

Funding/Support: Self-funded.

\section{Acknowledgement}

The present study is a part of the research programme, “Assessment of Quality of Life of Adult Cancer Patients during inpatient Chemotherapy Treatment (CT), or outpatient Radiotherapy Treatment (RT) in a tertiary care hospital of New Delhi, India”. This research was supportedand assisted by at:

Department of Medical Oncology and Radiation Oncology, Dr. B.L. Kapur Memorial Hospital, New Delhi, India.

Under the Supervision of:

Dr. S. Hukku (H.O.D. of Radiation Oncology Department),

Dr. B.L. Kapur Memorial Hospital,

New Delhi, India.

Dr. Amit Agarwal (H.O.D. of Medical Oncology Department),

Dr. B.L. Kapur Memorial Hospital,

New Delhi, India.

Under the Guidance of:

Dr. Renita Bhamrah (Texila American University),

E-mail:darpreetrenita@gmail.com 
Texila International Journal of Clinical Research

Volume 3, Issue 2, Dec 2016

\section{References}

[1]. Deshpande, P. R., Sheriff, M. K., Nazir, A., Bommareddy, S., Tumkur, A., Naik, A. N. (2013). Patientreported quality of life outcomes in Indian breast cancer patients: Importance, review of the researches, determinants and future directions. J Can Res Ther, 9 (1), 11-6. Doi: 10.4103/0973-1482.110341

[2]. Fobair, Patricia, L.C.S.W., Spiegel, David. (2009). Concerns about Sexuality after Breast Cancer. The Cancer Journal, 15(1), 19-26. doi: 10.1097/PPO.0b013e31819587bb

[3]. http://journals.Iww.com/journalppo/Abstract/2009/02000/Concerns_About_Sexuality_After_Breast_Cancer.5. aspx

[4]. Hughes, Mary, K., M.S., R.N. (2000). Sexuality and the Cancer Survivor: A SILENT COEXISTENCE. Cancer Nursing, 23(6), 477-482.

[5]. http://journals.lww.com/cancernursingonline/Abstract/2000/12000/Sexuality_and_the_Cancer_Survivor_A_S ILENT.11.aspx

[6]. Sunderam, S., Haider, S., Kumar, M., Kashyap, V., Bhushan, S.S., S.J. (2015). Assessment of quality of life of cancer patients attending oncology clinic in a tertiary care hospital of Jharkhand, India. International Journal of Community Medicine and Public Health, 3(1), 281-286.

[7]. http://www.ejmanager.com/mnstemps/109/109-1446880098.pdf?t=1468312514

[8]. WHOQOL Group. (1998). Development of the World Health Organization WHOQOLBREF quality of life assessment. Psychol Med, 28:551-8.

[9]. Zung, W. W. K. (1971). A rating instrument for anxiety disorders. Psychosomatics, 12(6), 371-379. Available at: https://psychology-tools.com/zung-anxiety-scale/

[10]. Zung, W. W. K. (1965). A Self-Rating Depression Scale. (1965). Arch Gen Psychiatry, 12: 63-70. Available at: https://psychology-tools.com/zung-depression-scale/ 


\title{
Patient's perspective of QOL with respect to different domains of Quality of Life in Cancer patients during inpatient Chemotherapy Treatment or outpatient Radiotherapy Treatment in a tertiary care hospital of New Delhi, India
}

\author{
Article by Kavita Gupta \\ Ph.D. Clinical Research, Texila American University, India \\ E-mail: 16kavitagupta@texilaconnect.com
}

\begin{abstract}
Background: In recent times there had been increase in incidence of cancer in India as nearly two people were diagnosed every minute with cancer. This is mainly attributed to urbanization, industrialization, life style changes, population growth and increased life span. There is no current universally accepted definition for quality of life (QOL) as it takes into account many aspects of life which is very difficult to define and measure. Therefore, there is wide range of concepts and opinions about "quality of life" and, over time, these concepts had been turning to the patient's perception about the disease and treatment and its effects, in an objective and subjective way. Evaluation of quality of life was attempted in two ways, objective assessments done by the interviewer and subjective assessments completed by the patients themselves. Nowadays, QOL could be defined by the WHO as an individual's perception of their own position (experience) in life, in the context of the culture and value systems in their life and in relation to their goals, expectations, standards, and concerns. The proposed study was considered valuable since it emphasized on quality of life as one of the possible outcomes that stressed on the factor that patients with cancer often experienced a loss of control and feelings of helplessness, anxiety and depression during chemotherapy or radiotherapy treatment.

Objectives: This study was conducted to assess the Patient's perspective of QOL with respect to different domains of Quality of Life in Cancer patients during inpatient Chemotherapy Treatment or outpatient Radiotherapy Treatment sessions. The primary objective was to analyze subjective impacting factors of QOL in homogenous surviving cancer patients receiving chemotherapy or radiotherapy sessions. Because patients were the best source of information for QOL data as the patients are the ones who are experiencing the effects of the illness and treatment, therefore they might provide a unique perspective on how QOL could be better assessed and defined with respect to treatment regimens.

Methods: It was a cross-sectional, descriptive, hospital based evaluation study. Total duration of the study was 5 months (December 2015- April 2016), conducted in Medical and Radiation Oncology department of DR. B.L. Kapur Memorial Hospital, New Delhi, India. A convenience sample of 60 patients with cancer was selected. They were further divided into Chemotherapy group $(n=30)$ and Radiotherapy group $(n=30)$. Therefore, 30 cancer patients were on chemotherapy and the other 30 cancer patients were on radiotherapy. Data was obtained through direct interview, using validated Psychological intervention tool in the form of Questionnaire: WHOQOL-Bref Questionnaire, Zung Self-Rating Anxiety scale and Zung Self-Rating Depression scale, which was further managed through a statistical program, using appropriate statistical tests.

Results: A total of 60 cancer patients were included in the study in which Chemotherapy group consisted of 30n cancer patients and Radiotherapy group consisted of 30n cancer patients. In the study, 6(20\%) were males and 24(80\%) were females in the chemotherapy group, and, 15(50\%) were males and 15(50\%) were females in the radiotherapy group. Majority of the patients 32(53.34\%) were in the age range of 46-60 years. The study population had Breast cancer 33(55\%), Head cancer 6(10\%), and Neck cancer 21(35\%). Anxiety affected the Physical domain
\end{abstract}


$(p=0.007)$, Environmental domain $(p=0.036)$ in the Chemotherapy group and Social domain

$(p=0.016)$ in the Radiotherapy group. On the other hand, Depression affected the Social domain $(p=0.043)$ in the Chemotherapy group, and Social domain $(p=0.012)$ in the Radiotherapy group. Values of $p<0.05$ were considered to be statistically significant.

Conclusion: The cancer patients who completed this survey/research study were able to conceptualize their quality of life by clearly stating which areas of their life that was important to them and how satisfied they were with that area. It was observed that in the chemotherapy group, overall health was significantly correlated with the psychological domain $(r=-0.395 ; p=0.031)$, social domain ( $r=-0.429 ; p=0.018)$, and environmental domain $(r=-0.598 ; p<0.001)$ of the WHOQOL-Bref questionnaire. On the other hand, the radiotherapy group showed no significant difference.

Keywords: Cancer, Chemotherapy, Radiotherapy, Quality of Life, WHOQOL-Bref Questionnaire, Zung Self-Rating Anxiety scale, Zung Self-Rating Depression scale, Physical domain, Psychological domain, Social domain, Environmental domain, Patient-reported outcomes, Open-ended questions, Overall Health satisfaction.

\section{Abbreviations}

$\begin{array}{lll}\text { QOL } & : \text { Quality of life } \\ \text { QLQ } & : \text { Quality life Questionnaire } \\ \text { WHOQOL-Bref } & : & \text { World Health Organization Quality of life assessment-a short brief } \\ & \text { version } \\ \text { ZSAS } & : \text { Zung Self-Rating Anxiety scale } \\ \text { ZSDS } & : \text { Zung Self-Rating Depression scale } \\ \text { CT } & : \text { Chemotherapy Treatment } \\ \text { RT } & : \text { Radiotherapy Treatment; } \\ \text { DOM } & : \text { Domain } \\ \text { TPA } & : \text { Third Party Administrator } \\ \text { FNAC } & : \text { Standard Deviation. } \\ \text { SD } & \end{array}$

\section{Introduction}

Cancer is the second most common disease in India responsible for maximum mortality with about 0.3 million deaths per year ${ }^{[1]}$. In India, life expectancy at birth and risk of cancer had risen from 45 years in 1971 to 62 years in 1991 to 71 years expected by 2021-25 ${ }^{[2]}$. According to the International Agency for Research on cancer (IARC), a group chartered by the World Health Organization; cancers of the breast, head and neck were some of the most frequently occurring forms of cancer in both the male and female population of India ${ }^{[3]}$. India contributed up to $7.8 \%$ of the global cancer burden and $8.33 \%$ of global cancer deaths ${ }^{[4]}$. Cancer prevalence in India was estimated to be around 2.0 to 2.5 million, with over 7-8 lakh new cases detected every year and 4-5 lakh cancer deaths per year ${ }^{[5,6]}$. The incidence of cancer in Delhi was the fourth highest among Asian registries ${ }^{[7]}$. According to 
National Cancer Registry Program (NCRP) 2013 report, cancer was a threatening problem in India with an estimated 2.5 million people living with the disease with 19746 cases (29.8 \% of all cancer in men and $10.6 \%$ of all cancer in women) in Delhi alone ${ }^{[8,9]}$. According to Ferrans (1996) concept, Quality of life could only be defined by the individual in terms of particular period of time between the hopes and expectations of the individual's present experiences and lifestyle, past experience, hopes for the future, dreams and ambitions ${ }^{[10]}$. Patient-centered care theory postulated by the Institute of Medicine (IOM) in the year 2001 had been found to be associated with improved patient satisfaction, better treatment adherence, improved recovery and health outcomes, reduced readmission rates and better seeking of follow-up care ${ }^{[11]}$.

\section{Aims}

The main objectives of the present study was:

i. To determine the association and relationship of two standalone questions in WHOQOL-Bref regarding Overall QOL and Health satisfactionwith different domains of quality of life in cancer patients during chemotherapy treatment or radiotherapy treatment.

ii. To evaluate the patient's perspectives of QOL during Chemotherapy or Radiotherapy treatment regimen through close-ended questions that were asked in between the counseling session to understand the depth of QOL of cancer patients during the course treatment.

iii.To qualitatively analyze the patient's perspectives regarding QOL through subjective openended discussion.

\section{Patients and methods}

During the conduct of the proposed study Ethical Procedures were respected.After obtaining authorized Ethical approval (Ref. No.: IRB/AARCE/5/DEC/2015/1 and dated December $7^{\text {th }}$, 2015) for the research study protocol from IRB and Ethical committee from Dr. B.L. Kapur Memorial Hospital, New Delhi, patients and their caregivers were approached in the inpatient as well as, outpatient clinic, where the purpose of the study was explained and they were invited to participate. Patients who agreed to participate were asked to sign an Informed Consent Form followed by the implementation of the instrument in the form of questionnaires.

The RESEARCH DESIGN of the proposed approved study protocol included the following Sampling Technique:

1. Sample Size: The target population of patients undergoing Chemotherapy and Radiotherapy sessions.

Total participants $=60$ divided in the following pattern:

$\mathrm{N}$ (Chemotherapy treatment) $=30 \mathrm{n}$

$\mathrm{N}$ (Radiotherapy treatment) $=30 \mathrm{n}$

2. Eligibility criteria of the study:

\section{Inclusion criteria}

a. Breast cancer and sub-sites of head and neck tumors (e.g., nasopharyngeal, thyroid cancer, and parotid tumors)

b. Patients aged 18years or older,

c. Clinically diagnosed cases.

d. Cancer diagnosis confirmed by biopsy or FNAC,

e. Undergoing/during the treatment sessions

f. Voluntarily agreed to join the study.

g. Aware of diagnosis and predicted prognosis

\section{Exclusion criteria}

a. Inadequate clinical condition (ambulatory and terminally ill patients) to respond to an interview.

b. Have difficulty in understanding the questionnaire or communicating. 
c. Patients who were serious and didn't give consent were excluded from the study.

d. Had a history of psychiatric disorder

e. Choice of chemotherapy drugs and their dosage, irradiation dosage.

3. The study involved primary research by Individual face-to-face interviews with 60 cancer patients undergoing either chemotherapy or radiotherapy treatment sessions through Structured and Valid WHOQOL-Bref Questionnaire. Secondary data was collected by the researcher with the help of patient records available with the TPA department at Dr. B. L. Kapur Memorial Hospital, New Delhi.

4. Eligible patients were identified through an institutional database or by referring physicians and were approached at their simulation appointment. After giving written informed consent from the patient or their caregivers, participants completed the assessment that included the self-report measures.

5. Patients who agreed to participate were asked to sign an informed consent and then the instruments in the form of questionnaire were applied. Subjective areas were covered using case histories.

6. Special care of the potential risks due to emotional distress was taken care of so that their dignity was not harmed.

7. A total of 5-6 interviews were performed per week, in the time and days most suitable for the clinic in the months of December 2015 to April 2016.

8. Each interview lasted approximately 25-60 minutes and all patients were thanked for their participation, valuable time and information in the end.

\section{Results}

\section{(a) Data collection}

Treatment-related symptoms were assessed using a series of interviews through standard questionnaires of WHOQOL-Bref Questionnaire, ZSAS and ZSDS ${ }^{[12,13,14]}$.The questionnaire was provided in a language that the patient could understand (English / Hindi) followed by interview of the patient who was either undergoing chemotherapy or radiotherapy treatment sessions.

i. WHOQOL-Bref Questionnaire: This is an abbreviated version of the instrument WHOQOL100.It consisted of 2 parts- one aimed at the sociodemographic and health aspects and the other at the Quality of Life ${ }^{[12]}$. The latter consisted of 26 questions, being two about quality of life in general and other 24 representing each of the facets that made up the original instrument ${ }^{[12]}$. The questions were organized in 4 domains:

a. Physical domain (DOM1): It included7 questions pertaining to sleep, energy, mobility, the extent to which pain prevents performance of necessary tasks, the need for medical treatment to function in daily life, level of satisfaction with their capacity for work ${ }^{[12]}$.

b. Psychological domain(DOM2): It included6 questions pertaining to the ability to concentrate, self-esteem, body image, spirituality i.e. the extent to which they feel their life is meaningful, the frequency of positive or negative feelings i.e. blue mood, despair, anxiety, depression ${ }^{[12]}$.

c. Social domain (DOM3): It included 3 questions pertaining to satisfaction with personal relationships, social support systems and sexual satisfaction ${ }^{[12]}$.

d. Environmental domain (DOM4): It included 8 questions related to safety and security, home and physical environment satisfaction, finance i.e. does the respondent have enough money to meet their needs, health/social care availability, information and leisure activity accessibility and transportation satisfaction ${ }^{[12]}$.

In addition to the 4 domains, the WHOQOL-Bref included two stand-alone questions, one pertaining to the respondents' rated QOL, and other related to their Satisfaction with Health that were analyzed separately ${ }^{[12]}$. The score of each question ranged from 1 to 5 and higher scores indicated a better evaluation.Raw scores of the respective domains were then transformed from 0-100 with the lowest score of zero and the highest score of 100according to accepted guidelines ${ }^{[12]}$. 
ii. ZSAS: Zung self-rating anxiety scale quality life questionnaire is a likert scale format (scoring on 1 to 4 scale) that was built by a psychiatrist, William W. K. Zung to measure the rate of anxiety with 20 items self-reported examinations with 15 increased anxiety level questions and 5 (Q. no. 5, 9, 13, 17, 19) decreased anxiety questions that were answered by the patients ${ }^{[13]}$. Scores for each question ranged from 1 to 4 and higher scores indicated severe anxiety level. The raw scores were counted up and multiplied by 1.25 to reach a standardized score, according to the instructions that accompanied the scale ${ }^{[13]}$.

iii.ZSDS:Zung self-rating depression scale quality life questionnaireis a 20 items short selfadministered survey that was designed by William W. K. Zung to assess the level of four common characteristics of depression for patients: the pervasive effect, the physiological equivalents, other disturbances, and psychomotor activities. There were ten positively worded and ten negatively worded questions. Each question was scored on a scale of 1-4 (a little of the time, some of the time, good part of the time, most of the time) ${ }^{[14]}$. The higher scores indicated severe depression level. The raw scores were counted up and multiplied by 1.25 to reach a standardized score, according to the instructions that accompanied the scale ${ }^{[14]}$.

\section{(b) Statistical analyses}

The database and statistical analysis was performed using SPSS v.17 software. Descriptive statistics computation techniques were applied to the discrete and continuous data. Measures such as mean, standard deviation, minimum and maximum range were developed from the continuous data. Relative frequency was calculated for discrete data. Mean with standard deviation was used to summarize the age of patients. A p-value $<0.05$ was considered as statistical significant. Bivariate analysis was performed to assess the predictors of QOL. Based on the survey, Pearson Correlation coefficient denoted by $\mathrm{r}$ was calculated to determine the type of correlation, i.e., either positive or negative correlation among variables. Paired t-test was used to compare difference between score means of different domains.

\section{Socio-demographic characteristics of the patients}

Table 1. Socio-demographic characteristics in the form of Frequency and percentage of variables of the patients and correlation in the two groups, i.e., Chemotherapy and Radiotherapy

\begin{tabular}{|c|c|c|c|c|c|c|}
\hline \multirow[t]{2}{*}{ S.No. } & \multirow[t]{2}{*}{ Variables } & \multirow[t]{2}{*}{ Parameters } & \multicolumn{2}{|c|}{ Chemotherapy } & \multicolumn{2}{|c|}{ Radiotherapy } \\
\hline & & & $\begin{array}{l}\text { Frequency } \\
\text { (n) }\end{array}$ & $\begin{array}{l}\text { Percentage } \\
\text { (\%) }\end{array}$ & $\begin{array}{l}\text { Frequency } \\
\text { (n) }\end{array}$ & $\begin{array}{l}\text { Percentage } \\
(\%)\end{array}$ \\
\hline 1. & Gender & $\begin{array}{l}\text { a. Male } \\
\text { b. Female }\end{array}$ & $\begin{array}{l}6 \\
24\end{array}$ & $\begin{array}{l}20.0 \% \\
80.0 \%\end{array}$ & $\begin{array}{l}15 \\
15\end{array}$ & $\begin{array}{l}50.0 \% \\
50.0 \%\end{array}$ \\
\hline 2. & Age & $\begin{array}{l}\text { a. } 18-30 \text { years } \\
\text { b. } 30 \\
\text { c. } 46 \\
\text { d. } 60\end{array}$ & $\begin{array}{l}5 \\
17 \\
8\end{array}$ & $\begin{array}{l}16.7 \% \\
56.7 \% \\
26.7 \%\end{array}$ & $\begin{array}{l}6 \\
15 \\
9\end{array}$ & $\begin{array}{l}20.0 \% \\
50.0 \% \\
30.0 \%\end{array}$ \\
\hline 3. & $\begin{array}{l}\text { Marital } \\
\text { status }\end{array}$ & $\begin{array}{l}\text { a. Unmarried } \\
\text { b. Married } \\
\text { c. Widow } \\
\text { d. Divorced/ } \\
\quad \text { Legally } \\
\text { separated } \\
\text { e. Others }\end{array}$ & 30 & $100.0 \%$ & $\begin{array}{l}1 \\
28 \\
1\end{array}$ & $\begin{array}{l}3.3 \% \\
93.3 \% \\
3.3 \%\end{array}$ \\
\hline 4. & $\begin{array}{l}\text { Educationa } \\
\text { l status }\end{array}$ & a. Illiterate & 1 & $3.3 \%$ & 2 & $6.7 \%$ \\
\hline
\end{tabular}


Texila International Journal of Clinical Research

Volume 3, Issue 2, Dec 2016

\begin{tabular}{|c|c|c|c|c|c|c|}
\hline & & $\begin{array}{l}\text { b. Literate } \\
\text { i. Primary } \\
\text { ii. Secondary } \\
\text { c. Tertiary }\end{array}$ & $\begin{array}{l}4 \\
10 \\
15\end{array}$ & $\begin{array}{l}13.3 \% \\
33.3 \% \\
50.0 \%\end{array}$ & $\begin{array}{l}5 \\
11 \\
12\end{array}$ & $\begin{array}{l}16.7 \% \\
36.7 \% \\
40.0 \%\end{array}$ \\
\hline 5. & Occupation & $\begin{array}{l}\text { a. Service } \\
\text { b. Business } \\
\text { c. Housewife } \\
\text { d. Freelancers } \\
\text { e. Pensioners } \\
\text { f. Domestic } \\
\quad \text { duties } \\
\text { g. Cultivation }\end{array}$ & $\begin{array}{l}3 \\
3 \\
21 \\
3\end{array}$ & $\begin{array}{l}10.0 \% \\
10.0 \% \\
70.0 \% \\
10.0 \%\end{array}$ & $\begin{array}{l}10 \\
2 \\
11 \\
1 \\
4 \\
1 \\
1 \\
1\end{array}$ & $\begin{array}{l}33.3 \% \\
6.7 \% \\
36.7 \% \\
3.3 \% \\
13.3 \% \\
3.3 \% \\
\\
3.3 \% \\
\end{array}$ \\
\hline 6. & $\begin{array}{l}\text { Type of } \\
\text { family }\end{array}$ & $\begin{array}{l}\text { a. Nuclear } \\
\text { b. Joint }\end{array}$ & $\begin{array}{l}16 \\
14 \\
\end{array}$ & $\begin{array}{l}53.3 \% \\
46.7 \% \\
\end{array}$ & $\begin{array}{l}9 \\
21 \\
\end{array}$ & $\begin{array}{l}30.0 \% \\
70.0 \% \\
\end{array}$ \\
\hline 7. & $\begin{array}{l}\text { Cohabitant } \\
\text { s }\end{array}$ & $\begin{array}{l}\text { a. Living alone } \\
\text { b. Living with } \\
\text { partner } \\
\text { c. Living with } \\
\text { partner and } \\
\text { children } \\
\text { d. Living with } \\
\text { children } \\
\end{array}$ & $\begin{array}{l}15 \\
1 \\
14\end{array}$ & $\begin{array}{l}50.0 \% \\
3.3 \% \\
46.7 \%\end{array}$ & $\begin{array}{l}4 \\
5 \\
21\end{array}$ & $\begin{array}{l}13.3 \% \\
16.67 \% \\
70.0 \%\end{array}$ \\
\hline 8. & $\begin{array}{l}\text { Annual } \\
\text { income }\end{array}$ & $\begin{array}{l}\text { a. NA } \\
\text { b. } \leq 20 \\
\text { c. } 20 \\
\text { d. } 30 \\
\text { e. } 41 \\
\text { f. } \geq 84\end{array}$ & 22 & $26.7 \%$ & 19 & $63.3 \%$ \\
\hline 9. & $\begin{array}{l}\text { Place of } \\
\text { residence }\end{array}$ & $\begin{array}{l}\text { a. Small town } \\
\text { b. Big town }\end{array}$ & $\begin{array}{l}1 \\
29 \\
\end{array}$ & $\begin{array}{l}3.3 \% \\
96.7 \% \\
\end{array}$ & $\begin{array}{l}8 \\
22 \\
\end{array}$ & $\begin{array}{l}26.7 \% \\
73.3 \%\end{array}$ \\
\hline
\end{tabular}

(All tests were performed using Pearson $\chi 2$ test for association analysis)

\section{Clinical characteristics of the patients}

Table 2. Clinicalcharacteristics in the form of Frequency and percentage of variables of the patients and correlation in the two groups, i.e., Chemotherapy or Radiotherapy

\begin{tabular}{|l|l|l|l|l|l|l|}
\hline S.No. & Variables & Parameters & \multicolumn{2}{|l|}{ Chemotherapy } & \multicolumn{2}{l|}{ Radiotherapy } \\
\cline { 4 - 7 } & & & $\begin{array}{l}\text { Frequency } \\
\text { (n) }\end{array}$ & $\begin{array}{l}\text { Percentage } \\
\text { (\%) }\end{array}$ & $\begin{array}{l}\text { Frequency } \\
\text { (n) }\end{array}$ & $\begin{array}{l}\text { Percentage } \\
\text { (\%) }\end{array}$ \\
\hline 1. & Smoking habit & $\begin{array}{l}\text { a. Non-smoker } \\
\text { b. Ex-smoker }\end{array}$ & 27 & $90.0 \%$ & 27 & $90.0 \%$ \\
& & 3 & $10.0 \%$ & 4 & $10.0 \%$ \\
\hline
\end{tabular}


Texila International Journal of Clinical Research Volume 3, Issue 2, Dec 2016

\begin{tabular}{|c|c|c|c|c|c|c|}
\hline 2. & Drinking habit & $\begin{array}{l}\text { a. Non-drinker } \\
\text { b. Ex-drinker }\end{array}$ & 30 & $100.0 \%$ & $\begin{array}{l}26 \\
4\end{array}$ & $\begin{array}{l}86.7 \% \\
13.3 \%\end{array}$ \\
\hline \multirow[t]{2}{*}{3.} & Tobacco use & a. Yes & 1 & $3.3 \%$ & 7 & $23.3 \%$ \\
\hline & & b. No & 29 & $96.7 \%$ & 23 & $76.7 \%$ \\
\hline 4. & $\begin{array}{l}\text { Health } \\
\text { insurance }\end{array}$ & $\begin{array}{l}\text { a. Yes } \\
\text { b. No }\end{array}$ & $\begin{array}{l}23 \\
7\end{array}$ & $\begin{array}{l}76.7 \% \\
23.3 \%\end{array}$ & 19 & $\begin{array}{l}63.3 \% \\
36.7 \%\end{array}$ \\
\hline 5. & $\begin{array}{l}\text { Type of health } \\
\text { insurance }\end{array}$ & $\begin{array}{l}\text { a. Government } \\
\text { medically } \\
\text { insured } \\
\text { b. TPA } \\
\text { c. Cash }\end{array}$ & $\begin{array}{l}10 \\
13 \\
7\end{array}$ & $\begin{array}{l}33.3 \% \\
43.3 \% \\
23.3 \%\end{array}$ & 11 & $\begin{array}{l}40.0 \% \\
23.3 \% \\
36.7 \%\end{array}$ \\
\hline 6. & $\begin{array}{l}\text { Support by } \\
\text { charity } \\
\text { organization }\end{array}$ & $\begin{array}{l}\text { a. Yes } \\
\text { b. No }\end{array}$ & 30 & $100.0 \%$ & 30 & $100 \%$ \\
\hline 7. & $\begin{array}{l}\text { Cancer tumor } \\
\text { location }\end{array}$ & $\begin{array}{l}\text { a. Ca Breast } \\
\text { b. Ca Head } \\
\text { c. Ca Neck }\end{array}$ & $\begin{array}{l}23 \\
1 \\
6\end{array}$ & $\begin{array}{l}76.7 \% \\
3.3 \% \\
20.0 \%\end{array}$ & $\begin{array}{l}5 \\
15\end{array}$ & $\begin{array}{l}33.3 \% \\
16.7 \% \\
50.0 \%\end{array}$ \\
\hline 8. & $\begin{array}{l}\text { Disease } \\
\text { acceptance }\end{array}$ & $\begin{array}{l}\text { a. Yes } \\
\text { b. No }\end{array}$ & $\begin{array}{l}23 \\
7\end{array}$ & $\begin{array}{l}76.7 \% \\
23.3 \%\end{array}$ & $\begin{array}{l}15 \\
15\end{array}$ & $\begin{array}{l}50.0 \% \\
50.0 \%\end{array}$ \\
\hline 9. & $\begin{array}{l}\text { Reproductive } \\
\text { age of women }\end{array}$ & $\begin{array}{l}\text { a. Pre- } \\
\text { menopausal } \\
\text { b. Menopausal } \\
\end{array}$ & $\begin{array}{l}10 \\
14 \\
\end{array}$ & $\begin{array}{l}33.3 \% \\
46.7 \% \\
\end{array}$ & 9 & $\begin{array}{l}30.0 \% \\
20.0 \% \\
\end{array}$ \\
\hline 10. & Cancer type & $\begin{array}{l}\text { a. } \text { Primary } \\
\text { cancer } \\
\text { b. Recurrent } \\
\text { cancer }\end{array}$ & 30 & $100.0 \%$ & 30 & $100.0 \%$ \\
\hline \multirow[t]{2}{*}{11.} & $\begin{array}{l}\text { Co-existence } \\
\text { of metastasis }\end{array}$ & a. Yes & 8 & $26.7 \%$ & 30 & $100.0 \%$ \\
\hline & & b. No & 22 & $73.4 \%$ & & \\
\hline \multirow[t]{4}{*}{12.} & $\begin{array}{l}\text { Chemotherapy } \\
\text { 's cycle during } \\
\text { the interview } \\
\text { of QLQ }\end{array}$ & a. $1^{\text {st }}$ & & & & \\
\hline & & b. $2^{\text {nd }}$ & 2 & $6.7 \%$ & & \\
\hline & & c. $3^{\text {rd }}$ & 15 & $50.0 \%$ & & \\
\hline & & d. $4^{\text {th }}$ & 6 & $20.0 \%$ & & \\
\hline
\end{tabular}




\begin{tabular}{|l|l|l|l|l|l|l|}
\hline & & e. $5^{\text {th }}$ & 6 & $20.0 \%$ & & \\
\hline 13 & f. $6^{\text {th }}$ & 1 & $3.3 \%$ & & \\
\hline & $\begin{array}{l}\text { Radiotherapy' } \\
\text { s cycle during } \\
\text { the interview } \\
\text { of QLQ }\end{array}$ & a. $10^{\text {th }}-15^{\text {th }}$ & & & 5 & $16.7 \%$ \\
\hline & & b. $16^{\text {th }}-20^{\text {th }}$ & & & 21 & $70.0 \%$ \\
\hline & c. $21^{\text {st }}-25^{\text {th }}$ & & & 3 & $10.0 \%$ \\
\hline & d. $26^{\text {th }} 30^{\text {th }}$ & & & 1 & $3.3 \%$ \\
\hline
\end{tabular}

(All tests were performed using Pearson $\chi 2$ test for association analysis).

Paired t-test for two overall questions of WHOQOL-Bref in Chemotherapy group $(n=30)$

Table3: Paired $t$-test for two overall questions of WHOQOL-Bref in Chemotherapy group ( $\mathrm{n}=30)$

\begin{tabular}{|c|c|c|c|c|c|c|c|c|}
\hline & \multicolumn{4}{|c|}{ Paired differences } & \multirow[t]{3}{*}{$t$-test } & \multirow[t]{3}{*}{$r$-value } & \multirow[t]{3}{*}{ df } & \multirow{3}{*}{$\begin{array}{l}\text { Sig. (2- } \\
\text { tailed) }\end{array}$} \\
\hline & \multirow[t]{2}{*}{ Mean } & \multirow[t]{2}{*}{ SD } & \multicolumn{2}{|c|}{$\begin{array}{l}95 \% \text { CI of the } \\
\text { difference }\end{array}$} & & & & \\
\hline & & & Lower & Upper & & & & \\
\hline $\begin{array}{l}\text { Pair } 7 \\
\text { Q2-DOM2 }\end{array}$ & -15.66 & 2.38 & -16.55 & -14.77 & $\begin{array}{l}- \\
36.01\end{array}$ & $\begin{array}{l}-0.395 \\
p=0.031\end{array}$ & 29 & $p<0.001$ \\
\hline $\begin{array}{l}\text { Pair } 8 \\
\text { Q2-DOM3 }\end{array}$ & -5.50 & 1.52 & -6.06 & -4.93 & $\begin{array}{l}- \\
19.74\end{array}$ & $\begin{array}{l}-0.429 \\
p=0.018\end{array}$ & 29 & $p<0.001$ \\
\hline $\begin{array}{l}\text { Pair 2 } \\
\text { Q2-DOM4 } \\
\end{array}$ & -22.56 & 2.59 & -23.53 & -21.59 & $\begin{array}{l}- \\
47.62 \\
\end{array}$ & $\begin{array}{l}-0.598 \\
p<0.001 \\
\end{array}$ & 29 & p $<0.001$ \\
\hline
\end{tabular}

It can be concluded from the above table that in the chemotherapy group, overall health was significantly correlated with the psychological domain $(r=-0.395 ; \mathrm{p}=0.031)$, social domain $(\mathrm{r}=$ 0.429; $\mathrm{p}=0.018$ ), and environmental domain $(\mathrm{r}=-0.598 ; \mathrm{p}<0.001)$ of the WHOQOL-Bref questionnaire. On the other hand, the radiotherapy group showed no significant difference.

Means and standard deviations of the two global items of the WHOQOL-Bref administered to Chemotherapy $(n=30)$ and Radiotherapy $(n=30)$ cancer patients

Table 4. Overall QOL and mean QOL scores for both Chemotherapy $(\mathrm{n}=30)$ and Radiotherapy $(\mathrm{n}=30)$ groups

\begin{tabular}{|l|l|l|l|l|}
\hline \multirow{2}{*}{ Items } & \multicolumn{3}{|l|}{ Chemotherapy group } & \multicolumn{2}{l|}{$\begin{array}{l}\text { Radiotherapy } \\
\text { Group }\end{array}$} \\
\cline { 2 - 5 } & Mean & SD & Mean & SD \\
\hline Global Quality of Life & 2.80 & 0.40 & 2.80 & 0.41 \\
\hline Global Health & 2.66 & 0.53 & 2.67 & 0.54 \\
\hline
\end{tabular}

There was no such significant difference between the mean scores of Global QOL, and Global health in the chemotherapy as well as, radiotherapy group.

Overall QOL condition and frequency for both chemotherapy and radiotherapy groups 
Table 5. Overall QOL condition and frequency for both Chemotherapy $(\mathrm{n}=30)$ and Radiotherapy $(\mathrm{n}=$ 30)groups

\begin{tabular}{|l|l|l|l|l|}
\cline { 2 - 5 } \multicolumn{1}{c|}{} & \multicolumn{2}{l|}{ Chemotherapy } & \multicolumn{2}{l|}{ Radiotherapy } \\
\hline $\begin{array}{l}\text { Overall QOL answered by } \\
\text { patients }\end{array}$ & $\begin{array}{l}\text { Cases } \\
(\mathbf{n})\end{array}$ & $\begin{array}{l}\text { Frequency } \\
(\%)\end{array}$ & $\begin{array}{l}\text { Cases } \\
(\mathbf{n})\end{array}$ & $\begin{array}{l}\text { Frequency } \\
(\%)\end{array}$ \\
\hline Very poor & 5 & $16.7 \%$ & 0 & 0 \\
\hline Poor & 17 & $56.7 \%$ & 6 & $20.0 \%$ \\
\hline Neither poor nor good & 8 & $26.7 \%$ & 24 & $80.0 \%$ \\
\hline Good & 0 & 0 & 0 & 0 \\
\hline Very good & 0 & 0 & 0 & 0 \\
\hline
\end{tabular}

It could thus be depicted from the table that 5(16.7\%), and $17(56.7 \%)$ of the patients in the chemotherapy group experienced very poor and poor QOL respectively as compared to $6(20.0 \%)$ patients' poor QOL in the radiotherapy group.

\section{Health condition and frequency for both chemotherapy and radiotherapy groups}

Table 6: Health condition and frequency for both Chemotherapy $(n=30)$ and Radiotherapy $(n=30)$ groups

\begin{tabular}{|l|l|l|l|l|}
\cline { 2 - 5 } \multicolumn{2}{c|}{} & \multicolumn{2}{l|}{ Chemotherapy } & \multicolumn{2}{l|}{} \\
\hline $\begin{array}{l}\text { Health condition answered } \\
\text { by patients }\end{array}$ & $\begin{array}{l}\text { Cases } \\
(\mathbf{n})\end{array}$ & $\begin{array}{l}\text { Frequency } \\
(\%)\end{array}$ & $\begin{array}{l}\text { Cases } \\
(\mathbf{n})\end{array}$ & $\begin{array}{l}\text { Frequency } \\
(\%)\end{array}$ \\
\hline Very poor & 7 & $23.3 \%$ & 1 & $3.3 \%$ \\
\hline Poor & 10 & $33.3 \%$ & 8 & $26.7 \%$ \\
\hline Neither poor nor good & 11 & $36.7 \%$ & 21 & $70.0 \%$ \\
\hline Good & 2 & $6.7 \%$ & 0 & 0 \\
\hline Very good & 0 & 0 & 0 & 0 \\
\hline
\end{tabular}

It was observed that patients on chemotherapy [10(33.3\%)] had more poor QOL as compared to the patients on radiotherapy treatment $[8(26.7 \%)]$.

Co-relation between inpatient and outpatient medical services and co-operation for patientreported outcomes during chemotherapy or radiotherapy

Table 7. Patient-reported outcomes of subjective QOL during inpatient chemotherapy or outpatient radiotherapy sessions.

\begin{tabular}{|l|l|l|l|l|l|}
\hline S.No & \multirow{2}{*}{ Question/Facets/Item } & \multicolumn{2}{l|}{ Chemotherapy } & \multicolumn{2}{l|}{ Radiotherapy } \\
\cline { 3 - 6 } & & Yes & No & Yes & No \\
\hline $\mathbf{1 .}$ & $\begin{array}{l}\text { Hospital and primary doctor } \\
\text { co-operation }\end{array}$ & $26(86.7 \%)$ & $4(13.3 \%)$ & $23(76.7 \%)$ & $7(23.3 \%)$ \\
\hline $\mathbf{2 .}$ & $\begin{array}{l}\text { Information about future } \\
\text { problems. }\end{array}$ & $26(86.7 \%)$ & $4(13.3 \%)$ & $26(86.7 \%)$ & $4(13.3 \%)$ \\
\hline $\mathbf{3 .}$ & $\begin{array}{l}\text { Information about managing } \\
\text { potential relapse }\end{array}$ & 26(86.7\%) & $4(13.3 \%)$ & $\begin{array}{l}30 \\
(100.0 \%)\end{array}$ & 0 \\
\hline $\mathbf{4 .}$ & $\begin{array}{l}\text { Overall satisfaction with } \\
\text { hospital service }\end{array}$ & 28(93.3\%) & $2(6.7 \%)$ & $28(93.3 \%)$ & $2(6.7 \%)$ \\
\hline $\mathbf{5 .}$ & $\begin{array}{l}\text { Confident that the treatment } \\
\text { provided was the best possible }\end{array}$ & $29(96.7 \%)$ & $1(3.3 \%)$ & $27(90.0 \%)$ & $3(15.0 \%)$ \\
\hline $\mathbf{6 .}$ & Dietitian provided & $30(100.0 \%)$ & 0 & 0 & $30(100 \%)$ \\
\hline
\end{tabular}


Texila International Journal of Clinical Research

Volume 3, Issue 2, Dec 2016

\begin{tabular}{|c|c|c|c|c|c|}
\hline 7. & Counselor provided & $30(100.0 \%)$ & 0 & 0 & $30(100 \%)$ \\
\hline 8. & $\begin{array}{l}\text { your treatment is started as } \\
\text { soon after the diagnosis as you } \\
\text { would like }\end{array}$ & $15(50.0 \%)$ & $15(50.0 \%)$ & $24(80.0 \%)$ & $6(20.0 \%)$ \\
\hline 9. & $\begin{array}{l}\text { there are regular checks to find } \\
\text { new tumors, if your type of } \\
\text { cancer is heritable }\end{array}$ & $30(100 \%)$ & 0 & $30(100 \%)$ & 0 \\
\hline 10. & $\begin{array}{l}\text { your doctor consults other } \\
\text { doctors or refers you if } \\
\text { additional expertise is required }\end{array}$ & $30(100 \%)$ & 0 & $25(83.5 \%)$ & $5(16.7 \%)$ \\
\hline 11. & $\begin{array}{l}\text { doctors and nurses in the } \\
\text { hospital give you the best } \\
\text { possible care }\end{array}$ & $30(100 \%)$ & 0 & $30(100 \%)$ & 0 \\
\hline 12. & $\begin{array}{l}\text { you regularly receive } \\
\text { information about the effect } \\
\text { (advantages and } \\
\text { disadvantages) of the } \\
\text { treatment, during the treatment } \\
\text { period }\end{array}$ & $28(93.3 \%)$ & $2(6.7 \%)$ & $23(76.7 \%)$ & $7(23.3 \%)$ \\
\hline 13. & $\begin{array}{l}\text { Care-providers inform you } \\
\text { about patient organizations }\end{array}$ & 0 & $30(100 \%)$ & 0 & $30(100 \%)$ \\
\hline 14. & $\begin{array}{l}\text { you are informed if the waiting } \\
\text { time is longer than expected }\end{array}$ & $25(83.5 \%)$ & $5(16.7 \%)$ & $25(83.5 \%)$ & $5(16.7 \%)$ \\
\hline 15. & $\begin{array}{l}\text { you receive information from } \\
\text { the hospital about possibilities } \\
\text { for psychosocial guidance, } \\
\text { during treatment sessions }\end{array}$ & $10(33.3 \%)$ & $20(66.7 \%)$ & 0 & $30(100 \%)$ \\
\hline 16. & $\begin{array}{l}\text { the waiting time for inpatient } \\
\text { and outpatient sessions in the } \\
\text { hospital is less than } 15 \text { minutes }\end{array}$ & $30(100 \%)$ & 0 & 0 & $30(100 \%)$ \\
\hline 17. & $\begin{array}{l}\text { Was it possible to discuss a } \\
\text { second opinion with your } \\
\text { doctor? }\end{array}$ & $29(96.7 \%)$ & $1(3.3 \%)$ & $24(80.0 \%)$ & $6(20.0 \%)$ \\
\hline 18. & $\begin{array}{l}\text { Did the doctors listen carefully } \\
\text { to you? }\end{array}$ & $30(100 \%)$ & 0 & $30(100 \%)$ & 0 \\
\hline 19. & $\begin{array}{l}\text { If your cancer is heritable, was } \\
\text { examination of your relatives } \\
\text { discussed? }\end{array}$ & $15(50.0 \%)$ & $15(50.0 \%)$ & $5(16.7 \%)$ & $25(83.5 \%)$ \\
\hline 20. & $\begin{array}{l}\text { Do you have to wait too long } \\
\text { to get the first available } \\
\text { appointment for a test or } \\
\text { treatment? }\end{array}$ & $25(83.5 \%)$ & $5(16.7 \%)$ & $25(83.5 \%)$ & $5(16.7 \%)$ \\
\hline
\end{tabular}

The above table depicted some of the outcomes and necessities during the chemotherapy and radiotherapy sessions that was recorded while counseling and interviewing the cancer patients.

\section{Discussion}

The data was collected after each interview from the respondent to determine if new insights were being produced from each interview. Rich data with thick description consistent with data saturation was achieved after interviews with sixty participants. Patients had limited knowledge about the side effects of chemotherapy and radiotherapy treatment and their management. Therefore, different 
aspects of QOL were differentially important to individuals. Following were some of the open ended questions discussed with the patients.

1. What things in your life help to make your life meaningful?

2. Your description of quality of life?

3. What physical things (self and environment) help to make your life meaningful?

4. What physical things (self and environment) need to be changed to make your life worth living?

5. What psychological factors help to make your life meaningful?

6. What psychological things need to be changed to make your life worth living?

7. What social things help to make your life meaningful?

8. What social things need to be changed to make your life worth living?

9. How do you spend your time each day?

10. What things you would like to change in your day to help make your life worth living?

Some of the comments included for the above series of questions included:

1. "It's hard to evaluate yourself,"

2. "My biggest complaint to doctors is that warmth is needed for all of this to register,"

3. "This journey has brought me so much closer to the Lord,"

4. "The doctor can recite the most amazing facts, but it makes me feel alone and gives me no hope,"

5. "Most of the patients included in the study were home-makers and retired. So, they like to spend their each day with daily household chores",

6. "Mindfullness therapy in the form of meditation, yoga, walk, exercise, gardening, spiritual, watching television, spending time with the family were observed to be helpful in keeping the cancer patient either undergoing chemotherapy or radiotherapy psychologically occupied",

7. "Most of the patients needed counseling during the chemotherapy or radiotherapy sessions as they were found to be severely affected by anxiety and depression".

Furthermore, the Biblical view of illness and human suffering was found to be closely related to the origin of sin and was a natural component of finite embodied human beings ${ }^{[15]}$. Enduring adversity could be used to display the work of God in a person's life and to facilitate the development of Christian character and spiritual growth ${ }^{[15]}$. The eventual realization for most of the patients was that God is all-powerful and all-knowing, and he will reward those who do good unto Him ${ }^{[15]}$. Schub and Richards (2014), stated that spirituality was the vehicle needed to allow healthcare providers to identify interventions for improving patient physical and mental health leading to overall well-being [15]. According to the Ramanakumar study, a variety of practices related to religiosity like belief in god, belief in karma, increased temple visits, increased temple activities, visiting religious place, pilgrimage, Yoga, and the belief that 'god will save' were followed in India to overcome coping, improving mood, adjustment, and decrease in distressful symptoms such as loss of appetite and fatigue in cancer patients during the treatment regimen ${ }^{[16]}$. Studies of a Patanjali-based integrated Yoga program for patients with breast cancer developed by the Vivekananda Yoga Anusandhana Samsthana (VYASA) had consistently reported improvement in anxiety, symptom severity, and distress, nausea and vomiting, and effecton global QOL as well as beneficial effects on natural-killer cell counts and radiation-induced DNA damage ${ }^{[10]}$.According to a study conducted by Thrane in 2013, showed that Mindfulness-Based Stress Reduction (MBSR) which was a group program that included mindfulness practice in the form of Mindfulness meditation and listening to meditation CDs, hypnosis, mind-body techniques (breathing exercises, distraction, positive coping behaviors, and relaxation techniques), virtual reality, creative arts therapy (dance and movement, music, and art therapy), and massage were found to be effective and significant in improving QOL of cancer patients by preventing social decline, and reduced societal costs of persons living with cancer with an object oriented aim for experiencing life fully and being in touch with the full range of internal locus of human emotions and sensory experiences and paying attention to present-moment experience nonjudgmentally ${ }^{[17,18,19]}$. According to a study conducted by Neikrug, A. Bet al. in 2012 showed that 
Texila International Journal of Clinical Research

Volume 3, Issue 2, Dec 2016

morning administration of bright light improved and strengthens Circadian rhythms (CRs) which might protect women from experiencing CR deterioration during chemotherapy ${ }^{\text {[20] }}$.

\section{Conclusion}

Treating the patient holistically, both physically and psychologically, would lead to the best patient outcomes. During the Chemotherapy sessions, it was observed that 26(86.7\%) patients found the hospital and the primary doctor was cooperative, 26(86.7\%) patients were informed about the future problems, 26(86.7\%) patients were informed about managing potential relapse, 28(93.3\%) were overall satisfied, 29(96.7\%) were confident that the treatment provided was the best possible, $30(100.0 \%)$ were provided the counselor and dietitian, 15 (50.0\%) treatment was started as soon after the diagnosis, $30(100.0 \%)$ regular check ups to find new tumors, if the type of cancer was heritable, $30(100.0 \%)$ were satisfied from the doctor's consultation and nurses care, 28 (93.3\%) regularly received information about the effect (advantages and disadvantages) of the treatment, during the sessions,25 (83.5\%) were informed about the waiting time, 10(33.3\%) patients received for psychosocial guidance during treatment sessions, 30 (100.0\%) the waiting time for inpatient sessions in the hospital was less than 15 minutes, 15 (50.0\%) were interrogated for heritable cancer, 25 (83.5\%) waited long for the first appointment.

On the other hand, during the Radiotherapy sessions, it was observed that 23 (76.7\%) patients found the hospital and the primary doctor was cooperative, 26(86.7\%) patients were informed about the future problems, 30(100.0\%) patients were informed about managing potential relapse, 28(93.3\%) were overall satisfied, 27(90.0\%) were confident that the treatment provided was the best possible, 24 $(80.0 \%)$ treatment was started as soon after the diagnosis, 30 (100.0\%) regular check ups to find new tumors, if the type of cancer was heritable, 25 (83.5\%) were satisfied from the doctor's consultation and $30(100.0 \%)$ from nurses care, 23(76.7\%) regularly received information about the effect (advantages and disadvantages) of the treatment, during the sessions,25(83.5\%) were informed about the waiting time, 5 (16.7\%) were interrogated for heritable cancer, 25 (83.5\%) waited long for the first appointment.

However, it was observed in both the groups of patients that the care-providers did not provide any information regarding patient organization. Moreover, there was a scarcity of a dietitian and a psychosocial counselor for guidance in the radiotherapy group. Also, the waiting time for the radiotherapy sessions was more than 15 minutes.

In a nutshell, it could be concluded from the above observations that cancer patients on chemotherapy had more affected QOL rather than on radiotherapy treatment. Every individual patient had different aspects and definitions of QOL.

\section{Future directions}

The subjective questionnaires could be converted into likert scale type and then further pre-testing of the same is recommended with large number of sample size.

\section{Limitations of the study}

The only limitation of the present study was small number of sample size with single-centered study.

\section{Footnotes}

Author's Contribution: 1- acquisition of data; 2- analysis and interpretation of data; 3- drafting of the manuscript; 4- critical revision of the manuscript for important intellectual content; 5- statistical analysis; 6- administrative, technical and material supports.

Funding/Support: Self-funded. 


\section{Acknowledgement}

The present study is a part of the research programme, “Assessment of Quality of Life of Adult Cancer Patients during inpatient Chemotherapy Treatment (CT), or outpatient Radiotherapy Treatment (RT) in a tertiary care hospital of New Delhi, India”.This research was supportedand assisted by at:

Department of Medical Oncology and Radiation Oncology, DR. B.L. Kapur Memorial Hospital, New Delhi, India.

Under the Supervision of:

Dr. S. Hukku (H.O.D. of Radiation Oncology Department),

DR. B.L. Kapur Memorial Hospital,

New Delhi, India.

Dr. Amit Agarwal (H.O.D. of Medical Oncology Department),

DR. B.L. Kapur Memorial Hospital,

New Delhi, India.

Under the Guidance of:

Dr. Renita Bhamrah (Texila American University),

E-mail:darpreetrenita@gmail.com

Dr. Nilesh J. Patel (Texila American University)

E-mail: nileshcology127@yahoo.co.in

\section{References}

[1]. Ali, I., Wani, Waseem, A., Saleem, K. (2011). Cancer Scenario in India with Future Perspectives. Cancer Therapy, 8,56-70.

[2]. Bushan, D. K. (2014, November 2). Cancer scenario in India. Retrieved October 18, 2016, from Daily Excelsior: http://www.dailyexcelsior.com/cancer-scenario-india/

[3]. Bronson, T. (1999). "Quality of Life of the Hemodialysis Patient". Masters Theses. Paper 531. Retrieved from: http://scholarworks.gvsu.edu/theses/531

[4]. Booij, J. C., Zegers, M., Evers, P. M., Hendriks, M., Delnoij, D. M., Rademakers, J. J.(2013). Improving cancer patient care: development of a generic cancer consumer quality index questionnaire for cancer patients. BMC Cancer, 13, 203.

[5]. Coelho, K. R. (2012). Challenges of the Oral Cancer Burden in India. Journal of Cancer Epidemiology, vol. 2012, 17 pages. doi:10.1155/2012/701932

[6]. Deshpande, P. R., Sheriff, M. K., Nazir, A., Bommareddy, S., Tumkur, A., Naik, A. N. (2013). Patientreported quality of life outcomes in Indian breast cancer patients: Importance, review of the researches, determinants and future directions. J Can Res Ther, 9, 11-6. Doi: 10.4103/0973-1482.110341

[7]. Fjorback, L. O. (2012). Mindfulness and bodily distress. Dan Med J.59 (11):B4547.

[8]. Khandelwal, S., Kurady,B. L.,M. S. V., Asha, K.,James, G.,Bharti, C. (2015). Quality of life in cancer patients on chemotherapy.Journal of Applied Pharmaceutical Science, 4(5). 918-928.

[9]. Leyendecker, Mary, R. (2015). "Spiritual Quality of Life Among Geriatric Cancer Patients: A Descriptive Correlational Study". Master of Science in Nursing Theses. Paper 17.

http://digitalcommons.cedarville.edu/nursing_theses/17

[10]. Mansano-Schlosser, Cristina, T. \& Ceolim, Maria Filomena. (2012). Quality of life of cancer patients during the chemotherapy period. Texto \& Contexto - Enfermagem, 21(3), 600- 607. https://dx.doi.org/10.1590/S0104-07072012000300015

[11]. Manoharan,N., Tyagi, B.B., Raina, V. (2009). Cancer Incidences in Urban Delhi- 2001-05. Asian Pacific Journal of Cancer Prevention, 10, 799-806.

[12]. Neikrug, A. B., Rissling, M., Trofimenko, V., Liu, L., Natarajan, L., Lawton, S., Parker, B. A., AncoliIsrael, S. (2012). Bright light therapy protects women from circadian rhythm desynchronization during chemotherapy for breast cancer. Behav Sleep Med, 10(3):202-16. Doi: 10.1080/15402002.2011.634940. 
Texila International Journal of Clinical Research

Volume 3, Issue 2, Dec 2016

[13]. Ott, M. J., Norris, R. L., Bauer-Wu, S. M. (2006). Mindfulness meditation for oncology patients: a discussion and critical review. Integr Cancer Ther, 5(2), 98-108.

[14]. Ryan, M. (2015). "Quality of Life in Adolescents with Cancer". Honors Theses.Paper 2639. http://scholarworks.wmich.edu/honors_theses/2639

[15]. Singh, H., Kaur, K., Singh, Banipal, R. P., Singh, S., Bala, R. (2014). Quality of life in cancer patients undergoing chemotherapy in a tertiary care center in Malwa region of Punjab. Indian J Palliat Care, 20, 116-22. Doi: 10.4103/0973-1075.132627

[16]. Saha, D. (2016, June 1). Delhi has the highest cancer incidence in India. Retrieved October 13, 2016, from newslaundry.com: http://www.newslaundry.com/2016/06/01/delhi-has-the-highest-cancer-incidence-in-india/\#

[17]. WHOQOL Group. (1998). Development of the World Health Organization WHOQOLBREF quality of life assessment. Psychol Med, 28:551-8.

[18]. Yeole, B.B. (2007). Trends in incidence of head and neck cancers in India. Asian Pac JCancer Prev,8(4):607-12.

[19]. Zung, W. W. K. (1971). A rating instrument for anxiety disorders. Psychosomatics, 12(6), 371-379. Available at: https://psychology-tools.com/zung-anxiety-scale/

[20].Zung, W. W. K. (1965). A Self-Rating Depression Scale. (1965). Arch Gen Psychiatry, 12: 63-70. Available at: https://psychology-tools.com/zung-depression-scale/ 


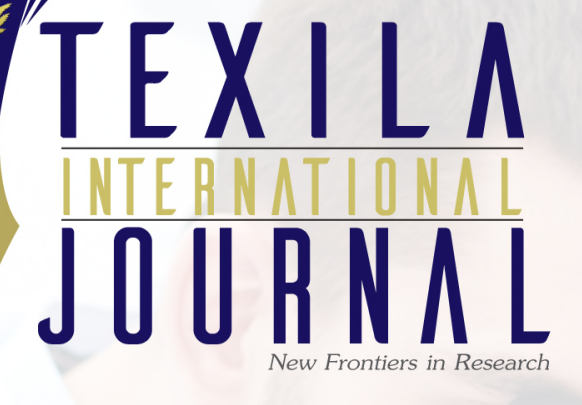

Texila American University, Providence, Georgetown, Guyana, South America.

Telephone: (+592) 2318118 / (+592) 2318111

E-mail: ejournal.assist@tau.edu.org Skype: texila.aco32 Whatsapp: +918056580933 